\title{
Clinical and molecular genetic studies in hereditary hair loss
}

Citation for published version (APA):

van Steensel, M. A. M. (2005). Clinical and molecular genetic studies in hereditary hair loss. [Doctoral Thesis, Maastricht University]. https://doi.org/10.26481/dis.20050309ms

Document status and date:

Published: 01/01/2005

DOI:

10.26481/dis.20050309ms

Document Version:

Publisher's PDF, also known as Version of record

\section{Please check the document version of this publication:}

- A submitted manuscript is the version of the article upon submission and before peer-review. There can be important differences between the submitted version and the official published version of record.

People interested in the research are advised to contact the author for the final version of the publication, or visit the DOI to the publisher's website.

- The final author version and the galley proof are versions of the publication after peer review.

- The final published version features the final layout of the paper including the volume, issue and page numbers.

Link to publication

\footnotetext{
General rights rights.

- You may freely distribute the URL identifying the publication in the public portal. please follow below link for the End User Agreement:

www.umlib.nl/taverne-license

Take down policy

If you believe that this document breaches copyright please contact us at:

repository@maastrichtuniversity.nl

providing details and we will investigate your claim.
}

Copyright and moral rights for the publications made accessible in the public portal are retained by the authors and/or other copyright owners and it is a condition of accessing publications that users recognise and abide by the legal requirements associated with these

- Users may download and print one copy of any publication from the public portal for the purpose of private study or research.

- You may not further distribute the material or use it for any profit-making activity or commercial gain

If the publication is distributed under the terms of Article $25 \mathrm{fa}$ of the Dutch Copyright Act, indicated by the "Taverne" license above, 


\title{
Clinical and molecular genetic studies
}

\author{
in hereditary hair loss
}

\section{PROEFSCHRIFT}

Ter verkrijging van de graad van doctor aan de Universiteit Maastricht, op gezag van de Rector Magnificus, prof. mr. G.P.M.F. Mols, volgens het besluit van het College van Decanen, in het openbaar te verdedigen op woensdag 9 maart 2005 om 16:00 uur

Door

Maurice Adrianus Monique van Steensel 
Promotores

Prof. dr. P.M. Steijlen

Prof. dr. dr. P.C.M. van de Kerkhof (UMC Nijmegen St Radboud)

Co-promotor

Dr. M. van Geel

Beoordelingscommissie

Prof. dr. F.C.M. Ramaekers (voorzitter)

Dr. C. Jahoda (University of Durham)

Prof. dr. E.C.M. Mariman

Prof. dr. C.S. Munro (University of Glasgow)

Prof. dr. C.T.R.M. Schrander-Stumpel

ISBN: 90-5681-217-3

Het onderzoek beschreven in dit proefschrift is verricht met steun van: Barrier Therapeutics NV, ZON-MW, Berliner Stiftung für Dermatologie, Pachyonychia Congenita Foundation, Stichting de Drie Lichten, Stichting Huidfonds en Merck Sharpe \& Dohme BV. 
Table of contents 


\section{Introduction}

\section{Chapters}

1. Molecular genetics of hair follicle growth and development

2. The gene for Hypotrichosis of Marie Unna maps between D8S258 and D8S298: exclusion of the hr gene by cDNA and genomic sequencing

3. A novel connexin26 mutation in a patient diagnosed with keratitisichthyosis-deafness syndrome

4. HID and KID syndromes are associated with the same connexin26 mutation

5. Connexin30.3 (GJB4) is not required for normal skin function in humans

6. Clouston syndrome can mimic pachyonychia congenital

7. A phenotype resembling the Clouston syndrome with deafness is associated with a novel missense GJB2 mutation

8. A 2bp-deletion in the GJA1 gene is associated with oculo-dento-digital dysplasia with palmoplantar keratoderma

9. Does recessive erythrokeratoderma variabilis exist?

10. Cryptic splicing at a non-consensus splice-donor in a patient with a novel mutation in the Plakophilin-1 gene

11. A case of Rombo syndrome

12. Woolly hair, premature loss of teeth, nail dystropohy, acral hyperkeratosis and facial abnormalities: possible new syndrome in a Dutch kindred

13. New syndrome of hypotrichosis, striate palmoplantar keratoderma, acro-osteolysis and periodontitis not due to mutations in Cathepsin C

14. A third case of HOPP syndrome - confirmation of the phenotype

15. Hypotrichosis, Lymphedema of the legs and acral telangiectasias - new syndrome?

General discussion

References

Summary

Samenvatting

Publications 
"The stars are made of the same atoms as the earth." I usually pick one small topic like this to give a lecture on. Poets say science takes away from the beauty of the stars -- mere gobs of gas atoms. Nothing is "mere." I too can see the stars on a desert night, and feel them. But do I see less or more? The vastness of the heavens stretches my imagination -- stuck on this carousel my little eye can catch one-million-year-old light. A vast pattern -- of which I am a part -- perhaps my stuff was belched from some forgotten star, as one is belching there. Or see them with the greater eye of Palomar, rushing all apart from some common starting point when they were perhaps all together. What is the pattern, or the meaning, or the *why*? It does not do harm to the mystery to know a little about it. For far more marvellous is the truth than any artists of the past imagined! Why do the poets of the present not speak of it? What men are poets who can speak of Jupiter if he were like a man, but if he is an immense spinning sphere of methane and ammonia must be silent? -- Richard P. Feynman (1918-1988) 


\section{Introduction}

Hair culture, hair development, molecular genetics and diseases 


\section{What's so important about hair?}

The human hair, while pretty dull in comparison to that of other mammals or the feathers of birds, is an extremely important component of social interaction. Some areas of the body show denser hair growth than others and seem to be designed to grab our attention. Most will not be seen in everyday life as clothing usually covers them. The one area that is almost always visible is the scalp and the hair that is on it receives an inordinate amount of attention, perhaps as a consequence of our instinctive need to see hair.

In many cultures throughout history, the hairdo was and still is one of myriad ways in which to inform others of one's station in life and political, social or religious orientation. Having no hair at all is usually seen as undesirable despite efforts by Hollywood to position bald actors as sex symbols (Yul Brynner is a good example ${ }^{1}$ ) and the image of detachment from worldly matters presented by the depilated scalps of Buddhist monks. A recent survey held among women and non-balding men has shown that men with a full head of hair are seen as more virile, attractive and intelligent than men who are bald or balding ${ }^{2}$. What's more, the balding males themselves think along these lines ${ }^{3,4}$ and have been doing so since biblical times. In the story of Samson and Delila, a man's power is believed to reside (among other places) in his hair and we still seem to believe this.

Whatever the reasons for our infatuation with this skin appendage, we are confronted every day with commercials telling us that having beautiful hair, to be obtained through the use of shampoos containing all manner of often mysterious and always expensive additives, is the most important thing in life. The evolutionary basis for this perception is unclear. While it is true, that the state of the hair can reflect one's general health, there seems to be no clear correlation between the amount of hair on the scalp and success in life as measured by the ability to procreate and survive long enough to provide for the resulting children. Nevertheless, the scalp hair is tremendously important 
and no effort is usually spared when something untoward -like losing ithappens to it. A simple Google search for "hair" returns no less than $32,500,000$ hits, most of them commercial web sites peddling novel or not quite so novel means of growing hair where there used to be none. Many thousands of web sites are devoted to the restoration of hair growth via an impressive array of low-, high- and ultrahigh-tech methods. None of those seem to be entirely satisfactory or rational despite eons of medical and magical research. As a testament to human resolve in the face of the inevitable, one of the oldest medical recipes ever found describes a tincture that Egyptian males used to treat their balding pates. It contained, among other interesting ingredients, fried and subsequently pulverized dog's paws. It is hard to see the rationale behind this concoction but we, in a country where homeopathy is taken seriously by about $80 \%$ of the population ${ }^{5}$, should not be too quick to laugh it off as a superstition of the Dark Ages. Modern treatments are often not much better or more rational.

Fortunately, the human obsession with beautiful hair is not limited to women, balding males, cosmetics/pharmaceutical companies and quacks. Increasingly, (molecular) biologists and physicians alike are coming to appreciate the beauty of hair, not as a cosmetic accessory, but as a model system for all aspects of growth and development. It so happens that the hair follicle is an organ that shows continuous and cycling growth, remodelling and death during life. It is accessible and can suffer from hereditary diseases caused by a single gene. The latter disorders can lead to hair loss and structural abnormalities of hair and as such are experiments of nature that demonstrate the importance of a particular gene for normal hair growth. They tell us something about its normal function. The genes are there to be discovered by whoever takes an interest in them. During the past five years, many facets of the developmental biology of the hair follicle have been elucidated using genetic diseases as model systems. The components discovered vary from $p 53$ homologs to proteins required for cell adhesion and communication. The gene and protein networks that are emerging as 
regulators of hair follicle growth and differentiation are turning out to be highly complex and are becoming more complicated with every new discovery made. For each piece of the puzzle that we can add, two or more pieces are found to be still missing. 


\section{This review; some thoughts on the study of rare (hair) diseases}

In the following review of hair follicle genetics, it will be noted that several sub-processes are discussed in separate sections. This was done for conceptual reasons but also to emphasize the modularity of the processes. Hair follicle development and growth are not regulated in a linear manner. Rather, it consists of several interlocking processes. It is of interest to note that almost every gene or protein whose function is known was discovered as a result of research into the genetic basis of a hereditary human or murine disease. Very few proteins have been discovered by means of inductive reasoning. This review focuses on the processes of development rather than on its disorders, hence the proteins and genes receive more attention than their diseases. However, diseases are perhaps among the most important phenomena that biology has to offer.

By identifying and investigating rare "new" diseases, the scientist and the physician-scientist can contribute to our knowledge of hair development. The power of genetics in dissecting disease mechanisms is aptly illustrated by the knowledge gained from the study of hereditary cancer syndromes.The venerated James Paget made a most eloquent statement in this regard, more than a century ago: "We ought not to set them aside with idle thoughts or idle words about "curiosities" or "chances".

Not one of them is without meaning; not one that might not become the beginning of excellent knowledge" ${ }^{6}$. McKusick, in his Foreword to Bean's 1967 Rare Diseases and Lesions, enumerates the reasons for studying rare diseases. He lists four reasons "that rare conditions are, or should be of interest to physicians":

- Rare disorders can teach us much about the normal or about more common disorders.

- Rare manifestations are sometimes valuable clues to the existence of grave internal disease. 
- People have them.

- They are a break in the routine and "keep [the physician's] powers of observation from undergoing atrophy".

There are those who feel that the study of rare diseases is too costly, of academic interest only and not relevant for medicine as a whole. This myopic view of medical research seems to be rather prevalent among physicians these days and is unfortunate, for it is they who witness Nature's experiments every day. Nature does not have a conscience or an ethical committee; the diseases it has created are nasty ones that result in much grief and suffering for those unlucky enough to be affected by them. Nature is amoral (not immoral!). We cannot ourselves create such diseases in humans and making mice suffer through them is not a particularly nice thing to do either. Hence, whenever we are offered the opportunity to learn about biological pathways by studying one of Nature's experiments we should be quick to take it for we are offered a unique, perhaps once-in-a-lifetime, insight into the inner workings of human biology.

It is the author's firm hope that someday, all doctors will appreciate unique cases as equally valuable as large cohorts subjected to double-blind, placebocontrolled studies, t-tests and Kaplan-Meier survival curves. 


\section{Chapter 1}

Molecular genetics of hair follicle growth and

\section{development}

Some parts of this introduction were previously published in various journals ${ }^{\text {7-10 }}$ 


\section{Basic hair morphology}

While the regulation of hair follicle differentiation, development and growth is highly complex, the basic anatomy of the hair follicle is in comparison relatively simple. An understanding of it is required for the review that follows. Figure 1 shows the main components of a single hair follicle. It is important to realize that every part has a function, sometimes more than one. As such, the hair follicle is an organ in its own right.

Hair grows from follicles, invaginations of the skin epithelium. A central and conspicuous component is the dermal papilla at the follicle base (1a). This structure is vital for hair growth as it signals to the germinative part of the hair follicle $(1 b)$ to grow and differentiate during hair cycling. The cells in the bulb divide and differentiate, with the cells of the cortical layer forming the bulk of the hair keratin (1c). The cortex is surrounded by several sheaths of cells. The outer root sheath is continous with the surface epithelium $(1 \mathrm{~d})$. The sheaths have unique characteristics in terms of gene expression but their significance is uncertain. The outer root sheath is known to play an important role in hair follicle cycling (see the discussion on the HR gene). The bulge of the hair shaft $(1 \mathrm{e})$ is located near the insertion of the $\mathrm{m}$. arrector pili and is believed to be the place where skin stem cells reside ${ }^{11}$.

The hair cycle is a fascinating phenomenon whose eventual elucidation promises to teach us much about the intricacies of the regulation of growth and development. There are three phases: anagen, katagen and telogen. All phases can be further subdivided based on morphology, gene expression patterns and growth characteristics. For this review, an understanding of the main phases suffices.

During anagen, the hair shaft is growing. The hair bulb is in close proximity to the dermal papilla. The duration of anagen is highly variable and determines the length that the hair may eventually reach. In the human scalp, the anagen phase may last up to seven years. Anagen is followed by the relatively short 
catagen. This phase lasts about two weeks and is characterized by a constriction of the middle part of the hair bulb. The expanded base of the hair distal to the constriction becomes a "club" that ascends in the hair follicle tract, trailing an epidermal strand that is connected to the dermal papilla. The epidermal strand is formed by connective tissue sheath of the hair. During catagen, the dermal papilla also starts to ascend. Thus, some "false" hair growth occurs during catagen but it is caused by the upward migration of the hair. Towards the end of catagen the epithelial strand shortens progressively and almost disappears. The dermal papilla starts to migrate downward again and the telogen phase commences. This phase lasts a few weeks and is followed by ejection of the hair. Some authors consider this as a phase of its own called exogen ${ }^{12,13}$. Towards the end of the telogen phase, the stem cells from the bulge region migrate downward ${ }^{14}$, meet the papilla and start forming a hair anew. It will be evident from the above that telogen is not simply a resting phase. Furious activity is taking place, paving the way for regeneration of the hair follicle. 


\section{Hypotrichosis}

Complex systems can break down. If it happens in an organism, the result is a disease whose symptoms are the result of the absence or other dysfunction of a component of the system and the compensatory action taken by the intact parts. As such, symptoms are not to be understood solely in negative terms but can also seen as positive in the sense that they reflect and reveal components of a biological network that are not normally visible. An excellent example is fever. This symptom shows that we have a thermostat in our brains that regulates core body temperature.

When a complex system such as the hair follicle breaks down, the result is often loss, abnormal structure or absence of the hair. If the dysfunction is the result of a single gene defect, chances are that the hair follicle will never properly develop. As a result, hair growth is absent at birth and will either be disturbed later on or never occur at all. Reduced or absent hair growth as a result of a genetic defect is called hypotrichosis to distinguish it from the result of loss of hair that was previously normal as for instance in male pattern baldness or alopecia areata. Depending upon the nature of the defect, the hairs that do grow may experience of more or less atypical abnormalities such as defects of the cuticle, altered growth speed and altered chemical composition. The word hypotrichosis implies a negative symptom, i.e., the absence of something that should be there. With the above in mind, one might also see the symptom as a positive one. The absence or the abnormality of the hair is a sign that the hair follicle is no longer functioning normally, showing that the causative gene functions in an ectodermal appendage. The extent to which the genetic network that governs hair growth can compensate determines whether some hair growth will or will not still occur. The presence and nature of the hair abnormality may even suggest a candidate gene. A good example is the human equivalent of the murine nude phenotype. The mice are hairless and have a severe immune deficiency. The human gene was found because it was noticed that some young children suffering from 
immune deficiency had hypotrichosis and nail dystrophy. The insight that they resembled the nude mouse then prompted a search for the human homolog, resulting in the identification of the human winged helix-nude $(W H N)$ gene ${ }^{15}$. Upon identification of the gene, the hair follicle can serve as a model system in which to study its function. The follicle is an accessible organ that can be cultured in isolation. Moreover, for most human hair disorders there exists a mouse equivalent. As several genes that are involved in hair growth have important functions elsewhere, the hair follicle may also serve as a model system for other processes that depend upon the presence of the gene product. The potential is almost limitless, but as a self-renewing organ the hair follicle serves best as a tool to study epidermal differentiation and regulation as well as stem cell biology. The obviously important part played by gap junctions (see below) in hair follicle function is also establishing the hair follicle as a model system for the study of gap junction biology. 


\section{Initiation of hair follicle differentiation}

Hair follicle development is a complex process that involves myriad interactions between many proteins. Details of the events governing the early stages of hair follicle development are now emerging. Here, an outline is presented of what is known about the molecular events governing hair follicle differentiation. For details of expression patterns and "minor" genes not directly involved in establishing the hair and regulating its cycle the reader is referred to comprehensive reviews published elsewhere ${ }^{16}$.

Like all other ectodermal appendages, including specialised ones such as ears and eyes, the hair follicle starts out as an ectodermal placode. In essence, this is a circumscript area containing cells that have been assigned a specialised role. The hair follicle is no exception. Early in human embryogenesis, between 9 and 12 weeks of gestation, the skin forms so-called ectodermal placodes in the region of the eyebrows and the chin. Here, ectodermal cells aggregate and will ultimately differentiate into the hair follicle ${ }^{17}$. There is evidence that the Delta-Notch pathway, known for its role in Drosophila neurogenesis, is involved in the initial delineation of the placode ${ }^{17}$. By a process known as lateral inhibition, the cells that are destined to form the ectodermal part of the hair follicle are excluded from the remainder of the primitive skin or peridermis ${ }^{18}$. Why the placodes appear at a certain time is unclear. Perhaps a developmental "clock" is involved, like in somite development ${ }^{19}$. Shortly after the placode is separated from its surroundings, further differentiation is initiated. The placode signals to a small group of underlying mesenchymal cells to condense ${ }^{20,21}$. Cells in the basal layer of the periderm elongate and form the hair germ, which starts to grow downwards. The initiation of growth and differentiation is partly dependent upon the presence of the bone morphogenetic protein (Bmp) inhibitor Noggin* that counteracts the

\footnotetext{
* A note on nomenclature: mouse gene nomenclature is used throughout, unless the discussion specifically concerns Homo Sapiens. See also http://www.informatics.jax.org/mgihome/nomen/gene.shtml
} 
inhibition of growth by Bmp proteins secreted by the ectoderm and mesenchyme 22 . This system may be partly responsible for the maintenance of lateral inhibition and setting up the boundaries of the future hair follicle. Recent evidence from mouse research suggests that Wingless protein family (Wnt) signaling is involved around the time that the adhesion molecule Pcadherin is upregulated and E-cadherin is down-regulated in the placode ${ }^{23-28}$. Wingless was first described as a Drosophila mutant lacking wings; the protein was subsequently found in mice and turned out to be a vital mediator of many intra- and intercellular signaling processes. It was originally identified as the site of integration of the oncogenic Murine Mammary Tumor Virus-129-32. Cadherins are transmembrane molecules that form the core of adherens junctions 33,34 . They connect to alpha-catenin, which is related to vinculin and is required for interaction with the actin cytoskeleton ${ }^{35}$. Another catenin, B-catenin, is structurally related to the Drosophila segment polarity gene Armadillo ${ }^{35}$. It binds to E-cadherin but also functions as a transcription factor, connecting cell adhesion to transcriptional events as a signal transducer ${ }^{36}$. On its own, it is subject to degradation. It is part of a multiprotein complex containing Axin, PP2B, Gsk3ß and the "tumor suppressor"^ $^{\prime \wedge}$ protein APC ${ }^{37-39}$. If unchecked by an external signal, Gsk3ß phosphorylates $B$-catenin, marking it for degradation through the ubiquitin pathway ${ }^{40}$. However, upon receipt of a Wnt signal, the degradation machinery becomes inactive and $B$-catenin is stabilized. Wnt proteins bind to a receptor called Frizzled ${ }^{41}$. The signal passed on through the receptor requires an interaction between Dishevelled-like (Dishevelled is a fruit fly protein that determines the orientation of body segments) proteins and Axin ${ }^{42}$. Stabilized B-catenin binds to and activates members of the Tcf/Lef1 family of transcription factors ${ }^{43}$. Jamora et al. have recently demonstrated that Wnt3a (a skin-expressed Wnt protein) can induce Lef-1 expression by stabilizing B-catenin in cultured mouse keratinocytes when the Bmp

\footnotetext{
$\wedge$ "Tumor suppressor" is a misnomer. The normal function of the protein is of course not the suppression of tumorigenesis. The term is used here because it is customary to do so.
} 
antagonist Noggin is added to the culture ${ }^{11}$. Noggin is expressed by the dermal papilla, while Wnt3a is produced by the ectodermal placode. This requirement for Noggin is also present in vivo, as noggin -/- mice fail to form about two-thirds of their hair buds ${ }^{22}$. The combined Noggin/Wnt signal seems to function by negative regulation of E-cadherin expression through Lef1. The repression requires both Lef1 and 8 -catenin. The simultaneous upregulation of $P$-cadherin expression sets the stage for the remodeling of the hair germ. Many of the subsequent steps are still unknown, but the nature of the early players offers a clue as to how subsequent hair follicle development might take place. Mice lacking the Lef1 gene have a phenotype reminiscent of the human disorder scalp-ear-nipple syndrome (MIM 181270*)44-48. They lack body hair and whiskers and have underdeveloped mammary glands, illustrating the importance of Lef1 mediated signalling in the development of the hair follicle and confirming that the Wnt-B-catenin-Lef1 pathway is required for the development of skin appendages. That mammary glands are affected too shows that the building blocks of skin appendages are basically identical regardless of the nature of the appendage. The early steps in hair follicle morphogenesis are outlined in figure $2 \mathrm{a}$.

Lef1 can regulate the activity of a signalling pathway that is intimately connected to apoptosis although there does not seem to be feedback. A recently identified TNF- $\$$ family member, ectodysplasin (Eda), is a target of Lef1 signalling. Ectodysplasin, encoded by a gene on $\mathrm{Xq} 28$, has two slightly different conformations that bind to related but distinct receptors. The most important type, Eda-A1, interacts with Edar, a tumour necrosis factor receptor family member that is also known as Downless, because the mouse mutant lacks fur ${ }^{49-52}$. The Eda receptor requires an adaptor molecule called Edaradd. Doffinger et al. demonstrated that Dl triggers NF-kappa-B (NF $\kappa$ B) activation through the Nemo (N $\kappa B$ essential modulator) protein ${ }^{53}$. It is interesting to note that this signal cascade is involved in apoptosis as well as cell survival.

\footnotetext{
* MIM numbers refer to the disease entry in the Online Mendelian Inheritance in Man database at http://www.ncbi.nlm.nih.gov/omim
} 
NEMO is a so-called I-kappa-kinase (Ikk $\gamma$, or Ikbkg) encoded by a gene on

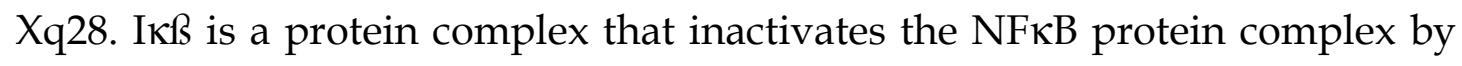
trapping it in the cytoplasm. I-kappa-kinases phosphorylate Iкß and mark it for ubiquitination, thus removing the inhibition on $\mathrm{NF} \mathrm{B}^{54}$. NFkB itself is a complex that can regulate several genes involved in inflammation and apoptosis. Depending upon the upstream signaling event, NFkB can either induce apoptosis or prevent $\mathrm{it}^{55,56}$. This balance depends in part upon the presence of the cylindromatosis (Cyld) gene, whose protein product Cyld targets Nemo for deubiquitination ${ }^{57-59}$. Apparently, too much NEMO activity is detrimental as CYLD mutations in humans lead to familial cylindromatosis or Brooke-Spiegler syndrome (MIM \#605041) $)^{60}$, demonstrating the functionality and importance of an intact NFKB pathway in the hair follicle. Inhibition of Cyld increases resistance to apoptosis, suggesting a mechanism through which loss of Cyld contributes to oncogenesis. It was shown that this effect can be relieved by salicylic acid and derivatives that inhibit NFKB activity ${ }^{57-59}$, which immediately suggests a therapeutic intervention strategy to restore growth control in patients suffering from familial cylindromatosis. Makris et al. showed that female mice heterozygous for Ikbkg (Nemo) deficiency develop a dermatopathy characterized by keratinocyte hyperproliferation, skin inflammation, hyperkeratosis, and increased apoptosis ${ }^{61}$. Although Ikbkg +/- females eventually recovered, Ikbkg -/males died in utero. Humans with defects in EDA-A1, its receptor EDAR or the adapter EDARADD suffer from X-linked hypohidrotic ectodermal dysplasia (Christ-Siemens-Tourraine syndrome, MIM \#305100) or autosomal dominant/recessive hypohidrotic ectodermal dysplasia (MIM \#129490/224900), respectively51,62,63. Patients suffering from these disorders lack hair and sweat glands and have defective teeth. In addition, they have dysmorphic features of the mid-face. A mouse mutant called Tabby has mutations in the mouse ectodysplasin gene ${ }^{56}$. Another mouse mutant called Downless ( $d l$ ) lacks a functional ectodysplasin receptor ${ }^{50,51}$. The human phenotype partly overlaps that of mice lacking the epidermal growth factor 
receptor (Egfr) gene and recent work has demonstrated that the Ectodysplasin-Edar complex probably interacts with the Egfr pathway since high doses of Egf can partly rescue the Tabby phenotype ${ }^{64}$. How this interaction takes place is currently unknown, but it is possible that the interaction is on the level of p38/MAP-kinase since both pathways can regulate this protein kinase although in an opposite manner65,66.

The symptoms of hypohidrotic ectodermal dysplasia are very similar to those of the human disease incontinentia pigmenti type 2 (IP2, Bloch-Sulzberger syndrome, MIM \#308300). Indeed, biopsies and cells from IP2 patients exhibit defective IKBKG expression but normal expression of IKK catalytic subunits. The symptoms of IP2 are more severe than those of hypohidrotic ectodermal dysplasia but are otherwise quite similar. IP2 is caused by inactivating $N E M O$ mutations ${ }^{67}$ and thus represents the human counterpart to the abovementioned Ikbkg +/- mouse. Human males affected by the $X$ linked mutation die in utero. Apparently, deficiencies in the EDA-EDAREDARADD-NFKB pathway may confer an increased sensitivity to apoptosis. The LEF1 signal thus never reaches the target cell; instead, the target cell dies. Observations made in cell cultures and in patients suffering from hereditary disorders affecting NFKB signaling support this view. Failure of EDA to bind to its receptor in tissue culture results in failure of the ectodermal placode to initiate its next stage of development, evagination into the mesoderm ${ }^{52}$. Thus, EDA seems to be required for the initiation of growth and differentiation of epidermal appendages that is made possible by the changes in E-cadherin expression. Interestingly, a recent report suggest that Eda may be required for the initiation of Bmp, Shh and Lef1 expression, suggesting that the interactions described so far may not be linear 68 . However, it is important to realize that proteins interact in networks that tend to break down upon the failure of one or more components. Hence, this finding probably means that early hair follicle development depends on a number of interlocking feedback systems in which Eda has a prominent function. This hypothesis is consistent with the possible requirement for Eda in cell survival that was discussed above. The 
same report implicated mesenchymal Activin A as an inducer of Edar expression and WNT as the inducer of Eda expression ${ }^{68}$. Activin A may be the long sought-after mesenchymal signal that induces the ectodermal placode to form. That Tabby mice lack body hair placodes is consistent with this hypothesis. It would be of interest to have conditional skin-specific activin A knockouts. Figure $2 \mathrm{~b}$ summarizes the rest of the hair follicle initiation pathways.

It is also of interest to note that a system that is very similar to the one described above is used in feather branching in birds ${ }^{69}$ showing the extraordinary amount of evolutionary conservation in the machinery of appendage development. In the feather as in the hair though, initiating growth is only the first step of development. 


\section{Polarity and growth}

Upon initiation of hair growth, the axes of the future hair follicle need to be established. As the follicle elongates, the lower end becomes bulbous and develops a deepening cavity that will enclose the dermal papilla. The ectodermal part of the hair follicle acquires directionality by growing towards the dermal papilla (the condensed mesenchyme underneath the ectodermal placode) in an oblique direction. The Bmp's mentioned previously play a role in the establishment of up versus down ${ }^{22}$. Proximo-distal (and left-right as well) polarity is probably established by Sonic Hedgehog (Shh). During normal development, Shh expression in the hair follicle is stronger on one side of the hair follicle (the side that the future hair grows away from) than on the other ${ }^{70}$. This differential expression gives rise to differential growth, resulting in orientation of the hair follicle along an antero-posterior axis. The Wnt-7a gene may also be involved ${ }^{71}$. Shh is a homolog of the Drosophila gene Hedgehog, a signalling molecule responsible for the establishment of segment polarity, regulation of limb growth and initiation of neural tube formation 72 . How the differential expression is brought about is still unclear, although the homeobox gene Cutl1 (a homolog of the Drosophila gene Cuticle) seems to be required for Shh expression and may be involved in determining which cells respond to Bmp or Wnt signalling with Shh production ${ }^{73}$. The mouse Shh knockout has a severe, lethal phenotype with limb and neural tube abnormalities. It is less well-known that this mouse also has abnormal hair growth ${ }^{74,75}$. The hair follicles that develop are abnormally large, whereas the dermal papilla is too small. Some differentiation of hair matrix cells takes place but hairs do not form. Apparently Sonic Hedgehog is required for proliferation of the dermal papilla; the effect is mediated by Gli2. The Gli2 gene is a mammalian homolog of the Drosophila segment polarity gene cubitus interruptus ${ }^{76}$. Shh binds to a receptor known as Patched1, or Ptch1 ${ }^{77}$. This transmembrane protein is interacting with a 7 -transmembrane signal 
transducer (probably G-protein coupled) called Smoothened (Smoh) ${ }^{78,79}$. The signalling process is complex. In the unbound state, Ptch inhibits Smoh function through an as yet unknown mechanism. Upon binding of Shh to Ptch1, Smoh is released from inhibition and inhibits Costal2 (Cos2) activity, probably via Fused $(\mathrm{Fu})$. Cos2 is a microtubule binding protein, connecting the cytoskeleton to a signal system in manner that is similar to the Ecadherin/B-catenin system. Target genes of the Shh signal system are among others En1 and Gli1-3, Bmp genes and Wnt genes. In humans, mutations in PTCH1 cause basal cell nevus syndrome (MIM \#109400) 80,81. The most prominent features of basal cell nevus syndrome are developmental disturbances and basal cell carcinomas, suggesting an intimate link between hair follicle development and oncogenesis. Indeed, it has been hypothesized that basal cell carcinomas arise from skin stem cells that are committed to a hair follicle fate (Tilli et al., in press). The available molecular evidence suggests that this is indeed true ${ }^{82}$. Abnormal hair has not been described as part of this phenotype although in our experience patients tend to have rather coarse and curly hair. The importance of $\mathrm{SHH}$ signalling in human hair development is made clear by disorders that affect the processing of the $\mathrm{SHH}$ protein. It is synthesized as a precursor that traffics to microsomes and undergoes cleavage of a signal peptide. Within the endoplasmic reticulum, it undergoes autoproteolytic cleavage into a $19 \mathrm{kDa}$-terminal product $(\mathrm{SHH}-$ $\mathrm{N})$ and a C-terminal product of $25 \mathrm{kDa}(\mathrm{SHH}-\mathrm{C})^{83-85}$. All of the known biological patterning activity resides in SHH-N86. The cleavage is autocatalytic, but a reaction intermediate is hydrolyzed by cholesterol that becomes attached to the C-terminus of SHH-N87,88. This unusual modification is required for the biological activity of SHH-N. There are some human hereditary disorders that elegantly demonstrate the importance of this process for normal development of the skin and hair. The ConradiHünermann-Happle syndrome (MIM \#302960) is characterized, among other symptoms by linear ichthyosis, coarse lusterless hair and linear alopecia ${ }^{89}$. It is caused by a deficiency of 3-beta-hydroxysteroid-delta(8), delta (7)- 
isomerase, a principal component of the cholesterol biosynthetic route. The coding gene, Emopamil-binding protein $(E B P)$, is located on $\mathrm{Xp} 11^{90-92}$. As a consequence of the mutation, cholesterol availability in affected cells is too low for normal SHH signaling. Because the disorder is X-linked dominant, affected females will show functional mosaicism as a consequence of lyonization ${ }^{93}$. Affected hair follicles either do not grow or show disturbed orientation, resulting in either linear alopecia or coarse hair; exactly what would be predicted from the role of SHH in hair follicle development. A related disorder is called CHILD syndrome (MIM \#308050). CHILD is an acronym for congenital hemidysplasia, ichthyosis and limb defects. It is caused by deficiency of a 3-beta hydroxysteroid dehydrogenase (NSDHL) that takes care of a step in cholesterol biosynthesis prior to that effected by the EBP protein ${ }^{94}$. As expected, symptoms can be more severe than in ConradiHünermann-Happle syndrome and include linear alopecia and again, coarse hair ${ }^{95}$. Interestingly, CHILD syndrome shows extreme lateralization of the symptoms. The known role of $\mathrm{SHH}$ in left-right determination ${ }^{96}$ may partly explain this phenomenon. CHILD syndrome thus represents a very important lesson about the development of symmetry and asymmetry in humans. Unfortunately, we do not yet understand it.

The regulation of genes such as En1 is another mechanism by which Shh regulates tissue polarity ${ }^{97}$. If the latter is over-expressed, normal tissue polarity is disrupted, resulting in ectopic hair growth ${ }^{98}$. That Shh can regulate the expression of Bmp and Wnt genes suggests the presence of a feedback loop in growth regulation. Shh is a secreted molecule that can signal over a distance of several cell diameters ${ }^{99}$. Hence, the signal may be used to precisely delineate growth zones. In the developing feather, Shh promotes cell death of barb ridges whereas Bmp promotes survival ${ }^{69}$. An intricate balance between death and growth thus sculpts the prospective feather barb. It is conceivable that a similar process is taking place in the developing hair follicle. The subsequent stages in hair development are far less well elucidated at the molecular level. 


\section{Follicle cycling and apoptosis}

Once established and fully differentiated, the hair follicle must initiate its cycle of continuous growth and remodelling. In contrast to many other mammals, humans do not moult regularly. Hair follicle cycling in humans is asynchronous although a seasonal influence on the length of different parts of the hair cycle has been noted ${ }^{100}$. The author is aware of two patients suffering from true seasonal hair loss. They may represent so-called atavistic mutations, where a more primitive phenotype emerges once modern regulatory mechanisms are out of the way. X-linked hypertrichosis (MIM 307150) is another fascinating example of an atavism ${ }^{101,102}$. Little is known about the regulation of hair follicle cycling in humans. It is probably influenced (as it is for example in sheep), by sex hormones, light and circadian genes. One hormone known to influence the hair cycle is prolactin. In mice, prolactin can induce a transition from anagen to catagen ${ }^{103,104}$. Since prolactin secretion is stimulated by light exposure ${ }^{105}$ it is logical to assume an influence of prolactin on seasonal variations of hair growth as seen in other mammals ${ }^{106-114}$. There are no firm data for humans, but it is of interest to note that one of the symptoms of hyperprolactinemia can be hair loss ${ }^{115,116}$. Perhaps unsurprisingly, another hormone involved in adapting the organism to the seasons, melatonin, also influences hair growth in various mammal species 117,118 . It will be of interest to examine the melatonin responses of human hair.

Global regulation of hair follicle cycling is still something of a mystery, but the molecules governing the transitions between the phases of the follicle cycle are now coming into view.

The molecular mechanisms of anagen initiation are now being charted and, perhaps unsurprisingly, they are the same as those that initiate the development of the hair during embryogenesis. In telogen skin, the dermal papilla shows strong Bmp4 expression. Bmp4 functions as an inhibitor of 
anagen; it was predicted more than ten years ago that telogen skin contains an inhibitor of anagen ${ }^{119}$. Upon transition to anagen, the dermal papilla, outer root sheath and hair germ start to express Noggin, thus antagonizing Bmp4 $4^{120}$. As demonstrated by Jamora et al., Noggin can, together with a Wnt signal, down-regulate E-cadherin expression and set the stage for remodelling of the hair follicle ${ }^{11}$. The entire sequence of early hair development is repeated in early anagen ${ }^{120-122}$. The events preceding the changes in Noggin expression are unknown. Plucking the hair or traumatizing it in another way is known to initiate anagen, preceded by apoptosis of cells remaining after removal of telogen hair follicles ${ }^{123,124}$. Chase and Eaton found that there is a minimum number of hairs that needs to be plucked for anagen to be initiated ${ }^{125}$. Apparently, some secreted factor that can be released by trauma initiates anagen if there is enough of it. Several proteins have been proposed as the signal but none have been confirmed as such ${ }^{16}$. Neurotrophin-3 has been implicated in anagen development as well ${ }^{126}$, suggesting that the central nervous system or parasympathetic nervous system can in some ways influence the hair cycle, too. Probably, a combination of external signals such as prolactin or melatonin combine with an internal "clock", perhaps analogous to circadian rhythm proteins, times the initiation of anagen. Several human clock genes, involved in the regulation of circadian rhythms, are expressed in skin and mucosa ${ }^{127,128}$. Their mRNA levels correlate with the cellcycle, such that high levels of hPER1 are followed by DNA synthesis ${ }^{127}$. The clock proteins may also be involved in regulating the initiation of anagen or the response of hair follicle cells to mitogenic signalling. Alternatively, an oscillating gene expression system such as the one that regulates somite identity may be involved19,129-133. Here, oscillating levels of the Lunatic Fringe gene and several members of the bHLH family (such as mammalian Hairy genes) are thought to regulate Notch expression ${ }^{134,135}$. Intriguingly, periodic Notch signalling depends on the presence of Wnt3a/ß-catenin signalling136. Wnt signalling is also involved in the initiation of the anagen phase as described above. These circular relationships suggest a central role for Wnt 
signalling in hair follicle growth and cycle control. The clock governing the hair follicle cycle may very well be a modified version of the one that patterns the early embryo.

During the hair cycle, there is intense remodelling of several parts of the hair follicle. Progression through the various phases of the cycle requires correct remodelling; if this process goes awry, hair loss may ensue. Indeed, careful study of a mouse and human hair loss phenotype has provided compelling evidence for a central role of the zinc-finger containing transcription factor HAIRLESS (HR). In 1998, Ahmad et al demonstrated that mutations in the HR gene cause the hereditary autosomal recessive disease atrichia universalis with papular lesions (MIM \#209500/203655)137,138. In an inbred Pakistani family, newborn children underwent ritual shaving at one week of age. Affected children, though born with hair, never grew any after the shaving. Hence, the first moult did not take place. Affected children were incapable of growing terminal hair or vellus hairs. On the scalp they developed papular lesions. Recent data and careful examination of patients shows that these lesions are not limited to the scalp but may also be found on the knees and elbows. Examination of similar lesions in the homologous mouse hairless mutant has demonstrated that these lesions are hair follicles that have degenerated to cysts filled with keratinous material. Panteleyev et al have convincingly demonstrated that this is caused by a failure of hair follicles to properly localise apoptosis during the anagen-catagen transition ${ }^{139}$. Mice that carry a mutation at the Hairless $(\mathrm{Hr})$ locus develop seemingly normal hair follicles but shed their hairs completely soon after birth. Their hair follicles degenerate into characteristic dermal cysts shortly after the onset of the first catagen. Instead of undergoing their normal catagen-associated involution, the hair bulb and central outer root sheath disintegrate into separate cell clusters, thus disrupting all epithelial contact with the dermal papilla. The now isolated hair follicle portions form cysts while re-growing. Some dermal papilla cells that normally do not undergo apoptosis also die. Loss of functional Hr leads to a premature, highly deregulated catagen. More recent 
work has shown that apoptosis occurs as early as the anagen-catagen transition and takes part in reorganising the hair follicle in such a way that the hair can be ejected without taking all of the bulb and root sheaths with it $^{140}$. It is of interest to note that analysis of the $\mathrm{Hr}$ function offers a glimpse into the regulation of hair follicle cycling - two independent regions of $\mathrm{Hr}$ mediate binding to thyroid hormone receptors ${ }^{141,142}$. Hr interacts with histone deacetylases, enzymes that modulate chromatin structure and thereby repress gene activity ${ }^{143}$. Hr appears to be functioning as a co-repressor. Thus, Hr may be involved in modulating the effects of thyroid hormone on hair growth. Perhaps this explains the hypotrichosis that is often observed in the context of hypothyroidism. To make matters even more complex, inactivating mutations of the vitamin D receptor (VDR) result in vitamin D resistant rickets (MIM \#277440) with a congenital atrichia that is identical to the one caused by $H R$ mutations, suggesting that the proteins interact with each other ${ }^{144}$. Indeed, it was recently demonstrated that the vitamin D receptor (VDR), a zinc finger protein that belongs to the same family as HR, interacts with the HR protein 145. The latter inhibits expression of VDR target genes. The hair phenotype resulting from inactivating $V D R$ mutations suggests that the HR/VDR complex represses a repressor of cycling. This type of negative regulation is not uncommon (the sonic hedgehog receptor functions in this way ${ }^{146}$ ) and the idea that the basic state of hair cycle progression is a "blocked" one is intriguing because it is quite compatible with Panteleyev's observations of aberrant apoptosis ${ }^{140}$. Using apoptosis as a kind of clock to time hair follicle cycling makes biological sense since apoptosis tends to be an all-out process that is itself not easily fine-tuneable whereas an apoptosis-blocking agent can be regulated precisely. Doubtlessly, many other proteins are involved in the anagen-to-catagen transition. For instance, injection of Tgf $\beta 1$ into anagen hair follicles of mice induces early apoptosis and entry into catagen ${ }^{147,148}$. Normal catagen follicles show co-localization of Tgf receptor-II and apoptotic hair follicle cells ${ }^{147}$, strongly suggesting a role for Tgf $\beta 1$ in normal hair follicle cycling. It is tempting to speculate that $\operatorname{Tgf} \beta 1$ is also active in adult life by 
taking part in regulating apoptosis. More recent results suggest that the perifollicular vasculature may also be involved in catagen induction ${ }^{149}$; a single report suggests that skin mast cells may actually regulate the hair follicle cycle ${ }^{150}$. Hair follicle cycle regulation is a highly complex process; the more we know about it, the more daunting the complexity will become. A systems biology approach that integrates extensive computer modelling of genetic pathways with molecular cell biology will eventually be needed to help us understand it 151. 


\section{Cycle length control}

Comparatively little is known about how the length of the different phases of the hair follicle cycle is regulated. This is an important issue: the length of the anagen phase determines the length that the hair can attain. The angora mutation in cats, mice and hamsters has been known since ancient times and has been thoroughly bred into these animals for the beautiful lustrous coats it generates. The phenotype in mice is caused by mutations that either delete or render dysfunctional the $F g f 5$ gene ${ }^{152}$. This member of the fibroblast growth factor family apparently limits the length of the anagen phase ${ }^{152}$, a behaviour that is entirely consistent with that of other fibroblast growth factors. In mice, the angora mutation lengthens the anagen phase by approximately three days ${ }^{153}$. Other than their name might suggest, most of them effect negative regulation of growth processes. For instance, FGF3 inhibits longitudinal bone growth ${ }^{154}$. Activating mutations in the target receptor FGFR3 are known to cause the disorders achondroplasia, hypochondroplasia and thanatophoric dwarfism ${ }^{155-158}$. In these skeletal dysplasias, growth has been severely restrained. Interestingly, the mutations lead to increased growth in another FGF target organ, the skin. Severe varieties of achondroplasia can be accompanied by acanthosis nigricans and activating mutations in FGFR2 can lead to severe acne159,160. It is highly likely that FGF5 has a similar mode of action in regulating the length of the anagen phase of the cycle and that it does so in human hair as well. The ability to induce an angora mutation in humans would be of considerable interest for cosmetics if the anagen phase can be lengthened without causing fragility of the hair. In angora mice, the increased anagen length comes at a price since the hair cuticle shows structural defects that can be expected to weaken the hair ${ }^{153}$.

In humans, long lustrous hair is appreciated very much and it is logical to assume that the angora mutation also affects humans (figure 3). It is tempting to speculate that the (recessive) mutation has been selected for in performing 
artists and fashion models. It may be of interest someday to conduct a survey for the presence of hypomorphic FGF5 alleles in them. 


\section{The HOX code and hair}

In segmented organisms such as humans and fruit flies, segment identity is determined largely by the so-called "HOX code". Several Drosophila genes that code for DNA binding proteins are involved in the regulation of segmentation and segment identity. Genes such as Antennapedia are involved in determining the identity of appendages; the Antennapedia mutant has legs on its head instead of antennae. Such transformations, where one segments obtains the identity of another, are called "homeotic" (from the Greek 'Opotos, meaning "of a similar nature"). The genes that can effect such transformations all share a 183-bp DNA binding domain that was named "homeodomain" or "homeobox". There are 39 vertebrate Hox homeobox genes that are arranged in four parallel loci (A, B, C, and D). The genes in each cluster can be aligned on the basis of homology within the homeobox to form so-called paralog groups. The paralog groups are numbered 1-13. The Hox genes from paralogs 1 through 8 can be related to specific $H O M-C$ genes such as Antennapedia ${ }^{161}$. The paralogs 9 through 13 appear to be equally related to the Drosophila Abdominal-B $(A b d-B)$ gene ${ }^{162}$. The interesting thing about the linear arrangement of the vertebrate Hox genes is that their expression in the vertebrate paraxial mesoderm reflects their arrangement on the chromosome. That is, the Hox genes that are $5^{\prime}$ in the cluster are expressed later and in more caudal areas than the genes that are $3^{\prime}$. This beautiful phenomenon is called co-linearity 163 and it is seen in all vertebrates with homeobox gene clusters. The $A b d-B$ gene in Drosophila specifies abdominal segments, its human paralogs specify caudal and abdominal structures, a truly stunning display of functional evolutionary conservation. The HOX code, then, is defined as the combination of Hox genes expressed at a particular time in a particular locus or segment. Segment identity is determined by the code. One of the most important discoveries regarding the homeobox genes in recent years has been that, in humans and in mice, they are also involved in determining the 
identity of appendages such as digits and the genital system ${ }^{164,165}$. For instance, dominant missense mutations in the human $A b d-B$ class HOXA13 gene can cause hand-foot-genital syndrome (MIM \#140000), a rare disorder characterized by abnormalities of distal limb patterning and Müllerian duct fusion ${ }^{166}$. Perhaps unsurprisingly, other studies have expanded the role of Hox genes even further and shown that some are expressed in skin, in a region-specific manner. In mice,the $A b d-B$ paralog $H o x c 13$, a very posterior member of the Hox-C complex, is expressed in hair follicles throughout the body as well as in vibrissae and, curiously, in the tongue, hinting at the possibility of evolutionary ties between hair and tongue development. Mice lacking Hoxc13 suffer from posterior vertebral defects, as expected. They also are born without vibrissae and later suffer from alopecia as a result of brittle hair. Hair follicles are formed normally, but the hairs break before they emerge $^{167}$. As it turned out, Hoxc13 regulates keratin gene expression ${ }^{168}$. The hair keratin promoters contain numerous putative Hox binding core motifs: TAAT, TTAT, and TTAC ${ }^{168}$. Quite surprisingly, Hox genes are apparently not involved in patterning the hair but in regulating the expression of its constituents. It is not clear how this makes sense from an evolutionary point of view. Hoxc13 seemingly violates the principle of co-linearity as it is expressed throughout the skin, but the first expression of this particular Hox gene in mice is seen during segmentation. There, co-linearity is respected. It seems as if the Hox genes were co-opted for other tasks and are utilized after being set up for use during segmentation ${ }^{169}$. The same applies to other Hox genes that have been put to use in other processes such as cardiovascular morphogenesis ${ }^{170}$. With the Hox genes involved in hair keratin regulation, it should be relatively straightforward for the genome to actually specify the mix of keratins in a hair by altering the HOX code. Although an unproven supposition, it is not unreasonable to speculate that regional differences in hair texture are due at least in part to variations in the keratin content as dictated by the HOX code. 


\section{Regional specificity-where to grow hair and make it look like it should}

Hair structure and growth pattern are not the same all over the body. Some areas lack hair altogether whereas in others, the hairs are paired with apocrine sweat glands and may play a role in spreading pheromones ${ }^{171,172}$. The basis for this regional specifity is not well understood. Variations in texture may be specified by the HOX code, but we are pretty much in the dark as to why, for example, we do not have hair on the palms of our hands. It is quite possible that the genes that are involved in inducing hair growth also impart some form of specificity. For instance, the mouse Tabby mutant selectively lacks zigzag and guard hairs in the coat. Auchene hairs, peri-anal hairs and vibrissae are relatively unaffected ${ }^{173}$. Obviously, different hair types have different requirements for their morphogenetic processes. Unfortunately, this last statement more or less sums up the current state of our knowledge.

The question of the simple presence or absence of hair can probably be answered a little more easily. The engrailed-1 (En1) knockout mouse shows a transformation of ventral paw structures to dorsal ones ${ }^{98}$. Not only are the tendons specified as dorsal ones, the normally bare ventral paws are hairy in this mouse. Engrailed-1 is a mammalian homolog of the Drosophila gene Engrailed. By now, it should not be a surprise anymore to learn that Engrailed is a homeobox gene that is essential for early patterning events in the developing fruit fly embryo. In Drosophila, Engrailed induces Hedgehog (Hh) expression in the posterior part of abdominal segments and in the posterior part of the wing imaginal disc (the ectodermal placode from which the wing develops) ${ }^{174}$. The Hh protein, in turn, diffuses to neighbouring cells where it induces expression of the Drosophila Bmp homolog Decapentaplegic and of Wingless $(W g)^{174}$. These specify the anterior boundary. It will be obvious to the reader that these events almost exactly mirror early events in mammalian hair 
follicle morphogenesis. As research progresses, more parallels will without doubt be found. Several other Drosophila genes involved in segment specification and growth turn out to have mammalian homologs that are involved in hair growth, limb development and so on (for example: cubitus interruptus and the GLI family, Patched and the mammalian PTCH genes). We will need to understand Drosophila development to truly understand hair follicle biology.

Meanwhile, in the En1 knockout mouse, the transformation of the ventral paw can now be understood from the Drosophila model as an expansion of Wingless expression. The phenotype associated with absence of Wnt-7a elegantly confirms this idea by showing dorsal-to-ventral transformations ${ }^{175}$. From experiments in the domestic chicken it appears that the dorsalizing activity of Wnt-7a in is mediated through the regulation of the LIMhomeodomain transcription factor Lmx-1. The human equivalent is associated with the hereditary disorder nail-patella syndrome (NPS, MIM \#161200), characterized by congenital absence of nails and patellae as well as kidney failure ${ }^{176}$. The absence of nails is likely to be the consequence of a patterning defect, where the fingertip ventral domain, probably specified by EN1 or a homolog thereof, has expanded to include the dorsal surface. The absence of patellae is likewise the possible result of a dorso-ventral patterning defect. From the above, one might expect patients to show an abnormal hair distribution but no hair defects have yet been described in nail-patella syndrome. This may be the consequence of an ascertainment bias or a true absence of abnormalities.

Nature uses the same tools over and over again. The same genes that specify where the hair will grow are also used to start growing the hair. Once the embryo has developed to term, they are used to maintain the hair follicle cycle. In our search for "master" genes that control the fate of the embryo, such circular mechanisms are unsatisfying. There must be some kind of beginning in the Cartesian sense. What genes determine where EN1 will be expressed and specify ventral regions? Though largely conjectural, it is highly 
likely that Drosophila again will be the key to understanding. In the fruit fly, the earliest establishment of pattern is mediated through maternal effect genes such as Bicoid177. Next, the antero-posterior polarity is further subdivided by gap genes such as Krüppel and Hunchback178, after which individual segments are sequentially established by pair-rule and segmentation genes bearing strange names, such as Fushi tarazu, Even-skipped and Engrailed. There does seem to be some kind of hierarchy and it is the author's opinion that we can fully expect to find a similar hierarchy of patterning genes, including maternal effect genes, in the human. Hence it can be predicted that hair-bearing regions are specified by the mammalian equivalents of the genes that specify regions of $E n 1$ and $W g$ expression in fruit flies. Fushi tarazu (Ftz) is one of them ${ }^{179}$. It belongs to the Antennapedia class of homeobox genes. In humans, "dorsal" apparently translates to "hairy" in many cases (this principle can be appreciated by paying a visit to any beach during summer), and given the amount of evolutionary conservation seen so far in these pathways, it is tempting to speculate that a gene or genes similar to Ftz is/are involved as a kind of master regulator. A PSI-BLAST ${ }^{180}$ search of the high-throughput genome sequencing (HTGS) and SWISSPROT databases (http://www.ncbi.nlm.nih.gov) with the protein sequence of the Drosophila Ftz mRNA finds no obvious orthologs, but it is interesting to note that several homeobox genes of paralog group 5 show significant homology extending into the FTZ domain that defines fushi tarazu. Human HOX genes may be involved in the specification of hair-bearing regions. A conditional knockout of (several of) the Hox genes in mouse skin would be of great value in addressing this proposition. 


\section{The cytoskeleton and intercellular adhesion}

Obviously, hair is more than just growing cells. Most of the visible part of the hair consists of heavily cross-linked (through sulphur bridges) alpha-keratins of the "hard" variety. Keratins are members of the intermediate filament protein family and have even been found in the lowly cephalochordate (invertebrate) Amphioxus ${ }^{181}$. Such an impressive degree of evolutionary conservation usually implies that the protein has a vitally important and nonredundant function and indeed, intermediate filament molecules are essential for maintaining the structural integrity of metazoan (multicellular organisms) cells. Intermediate filaments get their name from the fact that their diameter $(10 \mathrm{~nm})$ is intermediate between that of microfilaments such as actin $(6 \mathrm{~nm})$ and microtubules (around $20 \mathrm{~nm}$ ). Interestingly, special proteins belonging to the intermediate filament family, the nuclear lamins, are found in the nuclear envelope. The gene structure of lamins suggests that they may be the progenitors of the cytoplasmic intermediate filament proteins ${ }^{182}$. This observation allows for many interesting speculations about the evolution of metazoan eukaryotic cells. The construction of a nucleus was an obvious innovation, allowing the emergence of eukaryotes. Bacteria and other protozoa do not have a nucleus. How the eukaryotic ancestor acquired a nucleus is the subject of intense debate and will maybe forever remain an unanswered question. A 1996 paper by Gupta and Golding suggests a chimeric model ${ }^{183}$. "Chimeric" refers to an organism containing tissues from at least two genetically distinct parents. The Gupta-Golding model proposes that the first eukaryotic cell arose as the result of a fusion between a Gramnegative eubacterium (host) without a cell wall and an archaebacterium (symbiont). The nucleus appeared as the result of the folding in of the host's membrane around the engulfed cell and apparently allowed the construction of complex biparental genomes. Single-celled eukaryotes and plants are different. Their nuclei do have membranes and nuclear pore complexes but 
lack lamins and inner nuclear membrane proteins ${ }^{182,184}$. It would seem that these nuclear envelope proteins constitute another innovation that subsequently allowed for the evolution of multicellular non-plant organisms (metazoa), the first of which probably resembled modern sponges. Interestingly, the more complex the metazoan, the more lamins it seems to have. Caenorhabditis Elegans (a small nematode or roundworm) only has one lamin gene albeit one with unusual properties ${ }^{185,186}$. Vertebrates have some inner nuclear membrane proteins that are absent from invertebrates (the Lamin Associated Proteins (LAP)). Plants have nuclear membrane genes that have no homologs in other metazoan genomes, suggesting that they evolved their nuclear envelope separately. Hence, the emergence of a nuclear envelope is intimately linked to the emergence of multicellularity. Why multicellular life requires special nuclear envelopes will remain the subject of conjecture. The connection between developing a cytoskeleton and multicellularity is easier to understand in a teleological way. A single-cell organism does not require mechanical rigidity because, obviously, gravity and internal turgor will work together to impart some stiffness upon the cell. There is no danger of structural damage under the influence of gravity or the feeble forces exercised by the cell's motor proteins as they move. Multicellular organisms on the other hand will require an intra- and extracellular scaffold to prevent their entire structure from collapsing under the influence of gravity. The other stresses generated by the interaction with the physical environment must also be dissipated. The evolution of exo- and endoskeletons is a good example of adaptation to physical stress on the macroscopic scale.

On the microscopic scale, the intracellular scaffold or cytoskeleton has evolved to a high degree of efficiency and intricacy in the vertebrate integument. Skin, hair and nails require a great deal of mechanical strength and their cytoskeleton has evolved accordingly. In mammalian hair and skin, the most important cytoskeletal proteins are the $\alpha$-keratins. The alpha refers to the fact that the central rod domain of mammalian keratins is an $\alpha$-helix. In 
reptiles and their distant relatives the birds, the central domain of the main keratins is a $\beta$-sheet. The different tertiary (and probably quaternary) structures doubtlessly explain the structural differences between reptilian appendages and their mammalian counterparts. Keratins come in many sizes and types, but can be subdivided into acidic (type I) and basic (type II) groups. Their clustering in paralogous groups on chromosomes 17 and 12 respectively suggests that they evolved through duplication events from a single common ancestor. The acidic keratins are keratins (KRT) 9-20 and the hair keratin group Ha, the basic keratins are KRT8-18 and the hair keratin group $\mathrm{Hb}$. Interestingly, KRT 8 and 18 are the first to be expressed in embryogenesis and are thought to be the oldest of the lot in evolutionary terms. Again, it seems that embryogenesis recapitulates some aspects of evolution. The genes coding for keratins 8 and 18 are exceptional in that they are both in the type II cluster on chromosome 12, supporting the notion that the different keratins evolved through a series of duplications. Keratins are never alone - they exist as obligate heterodimers of one acidic and one basic keratin. Most keratins only have one partner but some promiscuity exists. K1 can pair with keratins 9 and 10 as demonstrated by our and other groups' finding that mutations in the $\mathrm{K} 1$ gene can lead to a palmoplantar keratoderma that is virtually indistinguishable from that caused by mutations in $\mathrm{K} 9$ (Terrón et al, unpublished data) ${ }^{187,188}$. The only difference is in the extent of the palmoplantar keratoderma - K1 keratoderma extends onto the proximal wrist crease since $\mathrm{K} 1$ is expressed up to that area. K9 keratoderma is limited to the palmar side of the hand and does not extend beyond the distal crease. The clinical phenotypes associated with keratin gene mutations have shown that keratins are highly tissue-specific, being expressed preferentially in circumscribed skin layers and areas. In the hair, trichocyte keratins form the bulk of the hair fiber. These specialized keratins are sulfur-rich, allowing the formation of disulphide bonds that strongly connect the molecules and impart significant elasticity and tensile strength upon the hair. A "perm" is possible because of these disulphide bonds. If they are broken by either 
heating or chemical disruption, the hair can be molded into a desired shape that may or may not be aesthetically pleasing. Cooling the hair will allow disulphide bonds to form anew, fixing the hair in its new shape. It will be obvious that repeated chemical and thermal attack on the keratins would lead to denaturation of the proteins with loss of tertiary structure and function. Hence, the result of the perm is often a weathered appearance to the hair, prompting the disappointed clients to again visit their hairdresser for repairs to their damaged coiffures. Mutations affecting trichocyte keratins can be expected to lead to hair abnormalities that closely mimic those seen after a failed perm. Indeed, in patients suffering from the hair disorder monilethrix, mutations in the trichocyte keratin genes $h H b 1$ and $h H b 6$ were first identified by Winter et al. in several unrelated pedigrees ${ }^{189,190}$. We recently demonstrated that a mutation in $h \mathrm{Hb} 3$ can also cause a classical monilethrix phenotype (van Steensel et al, in press). In monilethrix, the hair is brittle, doesn't grow long and has a weathered appearance. In all, it looks like a failed perm. Microscopic examination shows a beads-on-a-string appearance that lends the disease its name ("monile" means necklace). The spacing of the beads is strictly regular. We do not know why. Interestingly, a scarring alopecia leading to permanent baldness can occur in monilethrix. Apparently, the hard keratins are also an important component of hair follicle structural integrity. This does not necessarily mean that they are expressed in the cells of the inner or outer root sheaths. Those have their own keratins.

In epithelia, keratins are connected to protein complexes that reside in the cell wall and are called desmosomes. They firmly connect neighbouring cells to each other. By inserting into the desmosome, keratins in one cell can connect indirectly to those in another cell and form an intricate intermediate filament network that imparts mechanical strength upon the tissue ${ }^{191}$.

The importance of this scaffold in maintaining tissue integrity is vividly illustrated by the severity of the blistering disorders that result either from autoimmune attack on desmosome constituents or from congenital absence of a desmosome protein ${ }^{192}$. The composition of desmosomes changes with cell 
type and adapts itself to the functional requirements of the cell. In epithelia, desmosomes anchor keratin intermediate filaments to the cell membrane ${ }^{193}$. In other cell types such as Purkinje cells of the heart and meningeal cells they anchor desmin and vimentin intermediate filaments, respectively. Three protein families make up the main building blocks of desmosomes. These include the desmosomal cadherins (desmogleins and desmocollins), the armadillo family proteins (plakophilins and plakoglobin) and the plakins (desmoplakin, periplakin, plectin and envoplakin) ${ }^{191}$. Each family has its own defining protein motifs. Most of our current understanding of desmosome assembly comes from immuno-electron microscopy and in vitro reconstitution studies that departed from earlier work on adherens junctions. From these studies it was predicted that intermediate filaments bind to desmoplakin, which in turn binds plakoglobin that is associated with the desmosomal cadherins $^{194}$. The latter constitute the core of the desmosome. By and large, this model seems to be an adequate general description of desmosome structure. However, it has become increasingly difficult to map the interactions between the various proteins as more protein family members are being discovered. Also, lateral interactions rather than the linear chains described above seem to play an important role in determining desmosomal plaque mechanical strength. The extent of these interactions is largely unknown. Recent data also suggest that desmosomes may have other functions besides maintaining structural tissue integrity. For instance, the desmosome protein Plakoglobin can interfere with $ß$-catenin signaling by linking with Apc and with Lef/Tcf ${ }^{195,196}$. If overexpressed in Xenopus, plakoglobin can mimic $\beta$-catenin overexpression and cause axis duplication ${ }^{197}$. Plakoglobin is also capable of activating $c-m y c$ and of inhibiting apoptosis ${ }^{198}$. These findings could indicate that desmosomes can function as signal transducers and may as such be important for hair follicle morphogenesis. In what respects these signaling capabilities contribute to the skin phenotype observed in hereditary desmosomal disorders remains to be determined, but the study of the 
availabe mouse models may supply information that helps to answer this question.

Components that do not belong to any of these families may serve other functions ${ }^{199}$. To complicate matters even further, recent evidence suggests that members of a particular gene family may partly or completely take over the function of a missing protein. For instance, forced Desmoglein (Dsg) 3 expression in mouse skin can prevent the blistering that occurs upon injection of pemphigus foliaceus antibodies against $D s g 1^{200}$. In the blistering disorder pemphigus foliaceus, this happens in the oral mucosa, where enough Dsg3 is made to protect the mucosa from the blistering resulting from the autoimmune attack on Dsg1. It is an important observation that suggests that gene therapy for desmosomal disorders can take the form of stimulating expression of genes that can compensate for missing ones. Obviously, precise knowledge of protein interactions in vivo is a requirement for such therapy. Finally, clinical observations also indicate that there exist interactions not predicted from the model nor observed in vitro. Keratinocytes from patients with a PLAKOPHILIN-1 (PKP1) mutation show collapse of the intermediate filament network, indicating that PKP-1 interacts with keratins. This interaction can explain part of the phenotype caused by the mutations (chapter 10) ${ }^{201}$. From these observations, it is clear that in vivo model systems, e.g., knockouts, are urgently needed to complement in vitro research. At present there are only four known mouse models, one of which is a naturally occurring desmoglein3 null mutant called balding. The desmoplakin and plakoglobin mutants die in utero, precluding in vivo study 202,203. The lanceolate mutant is bald due to absence of functional desmoglein $4^{204}$. The balding and lanceolate mutants show that mutations affecting desmosomal components can lead to hair loss. In humans, mutations in the already mentioned PKP1 gene lead to ectodermal dysplasia-skin fragility syndrome (McGrath syndrome, MIM \#604536), a disorder characterized by progressive loss of sparse, curly hair as well as extreme fragility of the skin 201,205. DESMOPLAKIN1 (DSP1) mutations and deletion of JUNCTIONAL PLAKOGLOBIN1 (JUP1) lead to quite similar 
disorders characterized by woolly hair, palmoplantar keratoderma and heart rhythm disturbances with ventricular dilatation ${ }^{206,207}$. In the case of JUP1 deletions, the resulting disorder is also known as Naxos disease (MIM \#601214), because cases cluster on the Greek island of Naxos ${ }^{207}$. The disease caused by DSP1 mutations is also known as Carvajal-Huerta syndrome (MIM \#605676). 


\section{Communication}

While most of this review has focused on intracellular events and long-range communication, the discussion of desmosomes shows that what happens between neighbouring cells is quite important. It has become increasingly clear that skin and hair follicle cells are utterly dependent for their normal functioning on their ability to quickly exchange information with their neighbours. The differentiation events described above require a means for rapid intercellular communication. In Drosophila for instance, Wingless signalling in the foregut depends on the presence of innexins, the fruit fly equivalent of vertebrate gap junctions ${ }^{208}$. Indeed, it seems that a failure to establish rapid communication channels can result in sometimes dramatic malfunctions of skin and its appendages.

Gap junction or "connexin" genes code for 4-pass transmembrane proteins that can assemble to form hexameric aqueous channels or connexons in the cell membrane. Connexons in adjacent cells will pair to form an intercellular passage, the gap junction, that allows for quick transport of water, ions and small $(<1 \mathrm{kDa})$ molecules. The gap junction proteins form a large family of at least 24 proteins that are divided into three families based on sequence similarity. Gap junction assembly can be heterotypical, with different connexins making up a connexon, or homotypical, in which case the connexon is composed of one connexin type only ${ }^{209}$. The basis for this selectivity is poorly understood, as are the functional consequences. There is little doubt that channel composition is of vital importance for several aspects of its function. The connexins are named either for their molecular weight in kilodaltons (eg., connexin26, 30 and so on) or after their family affiliation (eg., GJA1, GJB6 et cetera). Both naming systems are used interchangeably in the literature. Gap junctions are found throughout the animal kingdom, mainly in tissues that require their constituent cells to be electrically coupled and engage in rapidly networked behavior. For instance, the hair cells in the 
cochlea are responsible for the mechano-electrical transduction of sound waves transmitted to the inner ear. Of vital importance for this function is the maintenance of a high intracellular potassium level throughout the hair cells210,211. The gap junctions between the hair cells allow a quick passage of potassium ions, maintaining a constant potassium concentration in all hair cells and effectively linking them into a syncytium. Similarly, in the Purkinje system in the heart, the gap junction channels effect electrical coupling and allow propagation of the sinus node signal212. In peripheral nerves, propagation of the action potential requires the presence of gap junctions ${ }^{213}$. It is not known what gap junctions do in the skin in a physical sense. There are some data suggesting that they are involved in the propagation of $\mathrm{Ca}^{2+}$ waves in the skin 214 . Considering the importance of $\mathrm{Ca}^{2+}$ in epidermal differentiation, the influence that gap junctions exert on skin differentiation may be mediated at least in part by their effect on $\mathrm{Ca}^{2+}$ signalling.

The developments in the field of intercellular communication have been rapid. Increasingly, clinical phenotypes are correlated with specific gap junction gene mutations, offering insight into the function of gap junctions in the skin. For instance, congenital hypotrichosis and thickening of the nails can be caused by disturbances of gap junction intercellular communication (chapter 6) ${ }^{215}$. Of particular interest in this regard are the gap junction genes GJB2 (connexin26) and GJB6 (connexin30). Mutations in the former can cause widely different phenotypes depending upon the exact nature of the mutation ${ }^{9}$. There is a strong genotype-phenotype correlation that is presently poorly understood. The keratitis-ichthyosis-deafness syndrome (KID, MIM \#148210) is caused by three different mutations in GJB2 - G12R (glycine to arginine), S17F (serine to phenylalanine) and D50N (aspartic acid to asparagine) 216,217 . See chapter 4 . KID syndrome is characterized by a severe erythrokeratoderma, keratitis, bilateral sensorineural deafness and a propensity to develop squamous cell carcinoma ${ }^{216}$. A scarring alopecia causes hair loss but there is also a congenital hypotrichosis. The hystrix-like ichthyosis-deafness syndrome (HID, MIM \#602540) is identical with KID, 
being associated with D50N 218. Curiously, a mutation that is very near G12, namely N14K (asparagine to lysine) causes a phenotype that differs considerably from KID syndrome (chapter 7) ${ }^{219}$. Hypotrichosis is part of it as are deafness and nail dystrophy, but there is neither keratoderma nor keratitis. Whence the difference is presently unclear although some recent data suggest that $\mathrm{D} 50 \mathrm{~N}$ is a gain-of-function mutation causing gap junctions that contain mutated connexins to signal too strongly. Perhaps this explains the hyperproliferation of skin observed in KID syndrome. However, GJB2 is known to interact with GJB6, which may also explain part of the phenotype. Also, there is evidence to suggest that interference with GJA1 (connexin43) may be responsible for the occurrence of skin symptoms, at least in the context of dominant GJB2 and GJB6 mutations ${ }^{220}$. The in vitro findings are supported by our recent identification of a deletion mutation affecting the Cterminus of GJA1, causing oculo-dento-digital dysplasia (MIM \#164200) with palmoplantar keratoderma (van Steensel et al., in press; chapter 8). Skin symptoms have never been described as a symptom in ODDD. However, all mutations described so far were missense mutations affecting the first intracellular and transmembrane domains of GJA1. It seems that the Cterminus of GJA1 is instrumental in some way in causing the skin symptoms. This finding suggests that an important interaction between GJB2, 6 and GJA1 may take place through the C-terminal domain of GJA1.

The dominant GJB6 mutations G11R (glycine to arginine), V17E (valine to glutamic acid) and A88V (alanine to valine) can cause Clouston syndrome (MIM \#129500), a highly variable phenotype of hypotrichosis, palmoplantar keratoderma and nail dystrophy 221,222. The variability is such that nail thickening may be the only symptom ${ }^{215}$. It is to be expected that several cases of simple hypotrichosis (for instance hypotrichosis simplex) or isolated twenty-nail dystrophy will turn out to be caused by GJB6 mutations. All available data suggest a vital role for gap junction communication in hair growth and development but it is not understood what this role is. An 
international collaborative effort to understand the consequences of gap junction mutations is currently underway. 


\section{Some recent developments and the need for a new publishing system}

The above review was by no means exhaustive. Some genes involved in the patterning of skin and its appendages were not mentioned because of their relatively small role in the whole. Others were not because their role wasn't known when writing the part of the review that they should be in. Some recent developments should be mentioned because they point to previously unknown participants in hair development or because they support some hypothesis presented above.

The hypotrichosis-lymphedema-telangiectasia syndrome (HLT, MIM \#607823) is a rare disorder, first described by us in 1998 and characterized by congenital hypotrichosis, lymphedema and vascular defects such as telangiectasias (chapter 15)223. We recently found recessive as well as dominant mutations in the SRY-box containing transcription factor SOX18 224. While it is not yet known why the mutations lead to hair loss, it was recently demonstrated that the vascular adhesion molecule VCAM-1 is a target of SOX18 regulation, explaining at least part of the phenotype ${ }^{225}$. Other targets of SOX18 are not yet known, but it is of interest to note that a related SOX gene product, SOX17, can negatively regulate the WNT-ßs-catenin-TCF pathway in Xenopus by binding directly to the armadillo repeats in B-catenin 226. Jamora et al have convincingly shown that the WNT pathway is essential for the earliest steps of hair follicle development ${ }^{11}$. It is likely involved in the control of hair follicle cycling as well. Thus, it is tempting to speculate that the absence of SOX18 leads to inappropriate activity of B-catenin with disturbances of the hair follicle cycle as a consequence. Elucidating the role of SOX18 and B-catenin in the control of hair follicle growth and cycling will be of great value for our understanding of hair follicle biology. Nail abnormalities are not part of the HLT syndrome, suggesting that SOX18 has a function that is unique to hair and vascular endothelia. 


\section{New syndromes?}

In molecular dermatology, the identification of new syndromes is of great importance as they point to as yet unidentified key players in the development of integumentary structures. The HLT syndrome that was mentioned above is an example.

An interesting "new syndrome" that will teach us much about epithelial patterning was recently reported in a Mexican family 227. Affected persons suffered from ulnar ray hypoplasia and palmar polyonychia, that is, the presence of nails on the palmar surfaces of the fingertips. Obviously, a normal nail is not supposed to be there. One of the reasons for this is polarization, the process by which dorsal and ventral identity of the limb is determined and that was explained above. The phenotype in this family is curiously similar to that of the En1 knockout mouse and may be caused by absence of EN1 or one of its interacting proteins. Elucidating its cause will be of great value for understanding nail patterning.

Nail growth can also be affected in hereditary disease as for instance in Clouston syndrome or the pachyonychias. These disorders demonstrate the importance of communication and structural integrity of the cytoskeleton. That there must be other proteins governing nail thickness is aptly demonstrated by the phenotype of HOPP syndrome (MIM 607658, chapter 14) 228. "HOPP" is an acronym of Hypotrichosis-Osteolysis-PeriodontitisPalmoplantar keratoderma. A peculiar keratoderma and acro-osteolysis are some of its most conspicuous symptoms, but of particular interest here are the nail abnormalities. From an early age, they are irregularly thickened, dark yellow and strongly curved. The nails look as if they are affected by a mycosis.

The entire symptom complex strongly resembles Papillon-Lefèvre/HaimMunk syndrome (PLS/HMS, MIM \#245000/\#245010)229,230 while sharing the osteolysis with pycnodysostosis"231,232 (“Toulouse-Lautrec's disease”, MIM \#265800). Both are cathepsin diseases. Cathepsins are proteolytic enzymes 
that are involved, among others, in lymphocyte and monocyte function by proteolytically activating enzymes involved in cellular killing, such as granzyme A. They process antigens for presentation by the class II major histocompatibility complex 233-235. Apparently, some also serve an important role in normal cornification as well but it is not yet known which. Perhaps they are required for normal desquamation by cleaving desmosomal structures, similar to the serine protease SPINK5 236-238. Several cathepsin genes were analyzed in HOPP syndrome but no mutations were found. Thus, the existence of HOPP syndrome points to an unknown cathepsin or a protein that closely interacts with the cathepsins that are expressed in skin and monocytic lineage cells and must have a pivotal role in nail growth and development.

\section{ArXiv for biosciences}

Current progress in molecular biology is such, that any written review is outdated by the time it appears in print or even online. Online papers, unfortunately, often appear long after they have been submitted due to the time-consuming review process. The lack of a system such as arXiv (www.arxiv.org) that accepts physics papers and publishes them online, allowing peer review and prompt experimental validation of the results by anyone who is capable of doing so, is seriously holding back the biological sciences. Progress is being sacrificed to the accretion of "scoops" and the gratification of egos and there are no signs of impending change in this regard. As long as impact factors and citation indices are used to measure the scientific "quality" of researchers in the biosciences the system will remain in place. 


\section{Aims of this thesis}

Considering the above, the aims of this thesis can now be stated as follows:

1. To demonstrate the genetic basis of different inherited diseases affecting the hair and other organ systems with a special focus on gap junction diseases;

2. To provide a thorough clinical description of the diseases caused by the various mutations that we found in the course of our research;

3. To describe new and/or rare syndromes that point to yet unidentified genes and their proteins that play key roles in normal hair development;

4. To provide a synthesis of current knowledge and indicate the direction that future research efforts may take. 
Chapter 2 


\section{The Gene for Hypotrichosis of Marie Unna Maps between D8S258 and D8S298: Exclusion of the $\boldsymbol{h r}$ Gene by cDNA and Genomic Sequencing}

Maurice van Steensel, ${ }^{1,2,{ }^{*}}$ Frances J. D. Smith, ${ }^{3,4,{ }^{*}}$ Peter M. Steijlen, ${ }^{1}$ Irma Kluijt, ${ }^{6}$ Howard P. Stevens, ${ }^{7}$ Andrew Messenger, ${ }^{9}$ Hannie Kremer, ${ }^{2}$ M. Giles S. Dunnill, ${ }^{10}$ Cameron Kennedy, ${ }_{10}^{10}$ Colin S. Munro, ${ }^{11}$ Valerie R. Doherty, ${ }^{12}$ John A. McGrath, ${ }^{8}$ Seana P. Covello, ${ }^{4}$ Carrie M. Coleman, ${ }^{3,4}$ Jouni Uitto, ${ }^{5}$ and W. H. Irwin McLean ${ }^{3,4}$

${ }^{1}$ Department of Dermatology and ${ }^{2}$ Department of Human Genetics, University of Nijmegen, Nijmegen, The Netherlands; ${ }^{3}$ Epithelial Genetics Group, Department of Molecular and Cellular Pathology, Ninewells Medical School, Dundee, United Kingdom; ${ }^{4}$ Epithelial Genetics Group, Department of Dermatology and Cutaneous Biology and ${ }^{5}$ Department of Dermatology and Cutaneous Biology and Department of Molecular Medicine and Molecular Pharmacology, Thomas Jefferson University, Philadelphia; ${ }^{6}$ Department of Clinical Genetics, Free University Hospital, Amsterdam; ${ }^{7}$ Academic Department of Dermatology, Royal London Hospital and ${ }^{8}$ St. John's Institute of Dermatology, St. Thomas's Hospital, Amsterdam; ${ }^{7}$ Academic Department of Dermatology, Royal London Hospital and ${ }^{8}$ St. John's Institute of Dermatology, St. Thom
Hospital, London; ${ }^{9}$ Department of Dermatology, Royal Hallamshire Hospital, Sheffield, United Kingdom; ${ }^{10}$ Department of Dermatology, Bristol Royal Infirmary, Bristol, United Kingdom; "'Department of Dermatology, Southern General Hospital, Glasgow; and ${ }^{12}$ Victoria Hospital, Kirkcaldy, United Kingdom

Summary

Hypotrichosis of Marie Unna (MU) is an autosomal dominant hair-loss disorder with onset in childhood. A genomewide search for the gene was performed in a large Dutch family using 400 fluorescent microsatellite markers. Linkage was detected with marker D8S258, and analysis of this family and a further British kindred with additional markers in the region gave a combined maximum two-point LOD score of 13.42, with D8S560. Informative recombinants placed the MU gene in a 2.4cM interval between markers D8S258 and D8S298. Recently, recessive mutations in the $h r$ gene were reported in families with congenital atrichia, and this gene was previously mapped close to the MU interval. By radiation-hybrid mapping, we placed the $h r$ gene close to D8S298 but were unable to exclude it from the MU interval. This, with the existence of the semidominant murine $h r$ allele, prompted us to perform mutation analysis for this gene. Full-length sequencing of $b r$ cDNA obtained from an affected individual showed no mutations. Similarly, screening of all exons of the $h r$ gene amplified from the genomic DNA of an affected individual revealed no mutations. Analysis of expressed sequences and positional cloning of the MU locus is underway.

Received February 22, 1999; accepted May 21, 1999; electronically published June 18, 1999.

Address for correspondence and reprints: Dr. Irwin McLean, Epithelial Genetics Group, Department of Molecular and Cellular Pathology, Ninewells Medical School, Dundee DD1 9SY, United Kingdom. E-mail: w.mclean@hgmp.mrc.ac.uk

*These authors contributed equally to this work.

(C) 1999 by The American Society of Human Genetics. All rights reserved. 0002-9297/99/6502-0016\$02.00

\section{Introduction}

Alopecia is a common genetic trait in humans, primarily affecting males, in the form of male pattern baldness (Dawber 1997). Although intrinsically benign, the cosmetic effect of alopecia is considerable, and, therefore, demand for novel treatments for baldness is correspondingly large. Recently, a small number of genes have been identified in which mutations produce human hair loss, either alone or in conjunction with other ectodermal defects.

A single-gene form of baldness, congenital atrichia (MIM 203655; also called "congenital atrichia with papular lesions”), has been described in the literature. Congenital atrichia is an autosomal recessive disorder causing complete loss of all hair, beginning at an early age (Ahmad et al. 1993). Recently, the congenital atrichia gene was mapped to chromosome $8 \mathrm{p} 22-\mathrm{p} 21$, and mutations were reported in the human homologue of the murine hair-loss gene, hairless $(b r)$, in a number of families (Ahmad et al. 1998a, 1998b; Cichon et al. 1998; Nothen et al. 1998; Zlotogorski et al. 1998). The $\mathrm{hr}$ mouse was originally described in 1926 (Brooke 1926), but it was not until recently that the murine gene was identified (Cachon-Gonzalez et al. 1994). The hairless protein is a putative transcription factor thought to be involved in the regulation of the hair cycle, although the precise molecular mechanisms have yet to be elucidated (Panteleyev et al. 1998b). The genomic organization of the human $h r$ gene has been recently described, including an alternate transcript that shows some degree of epidermal specificity (Cichon et al. 1998; Ahmad et al. 1999). Ahmad and colleagues extensively analyzed the tissue distribution of $h r$ expression (Ahmad et al. 1999).

Mutations in the hair keratins hH6b and hH1b have been shown to cause monilethrix, which is a structural 
A

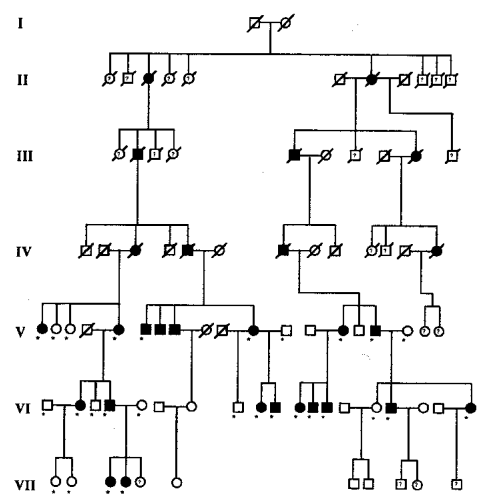

B

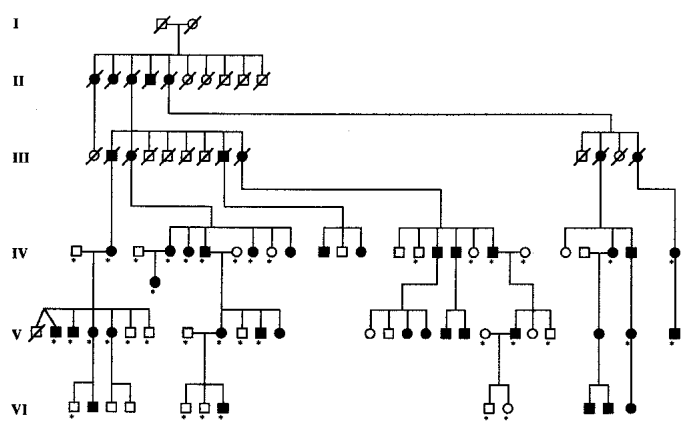

Figure 1 Pedigrees of two white families with MU that were used for linkage analysis, showing autosomal dominant inheritance. A, Pedigree of family 1 , who are of Dutch origin. B, Pedigree of family 2, who are of British origin. Asterisks (") indicate individuals from whom DNA was available for study.

disorder of the hair and often is accompanied by alopecia (Healy et al. 1995; Winter et al. 1997a, 1997b). In addition, a number of ectodermal dysplasia genes and loci have been identified in which alopecia is one of the epithelial defects. The conditions involved include X-linked ectodermal dysplasia, caused by mutations in the EDA gene (Kere et al. 1996); skin fragility/ectodermal dysplasia syndrome, caused by loss of plakophilin-1 expression (McGrath et al. 1997); Clouston syndrome, which maps to 13q11-q12.1 (Kibar et al. 1996); and Papillon-Lefevre syndrome, which has been recently mapped to 11q14 (Laass et al. 1997).

In 1925, the Hamburg-based dermatologist Marie Unna described a new type of autosomal dominant alopecia, which she had observed in an extended northern German pedigree (Unna 1925). This disorder is now known as "hypotrichosis of Marie Unna" (MU; MIM 146550). Later, Ludwig (1953) reexamined the same family, and, since these first descriptions, a number of others have appeared in the literature (Borelli 1954; Stevanovic 1970; Peachey and Wells 1971; Solomon et al. 1971; Bentley-Phillips and Grace 1979; Speigel and Hundeiker 1979; Wirth et al. 1985). MU is a rare disorder and is characterized by hair loss in a Norwood (or Hamiltonian) pattern (Dawber 1997). At birth, scalp hair is sparse, and the eyelashes and eyebrows are especially affected. During childhood, hair growth ensues, but the hairs that appear are coarse and wiry. In contrast to congenital atrichia-which appears, on the basis of the small number of cases so far studied, to result in complete hair loss in the early years of life-MU causes hair loss in the years close to the onset of puberty. Although eyebrows and body hair are somewhat affected in $\mathrm{MU}$, progressive alopecia of the scalp is the main feature of the disorder. Identification of the MU gene may give a valuable insight into the molecular-genetic mechanisms underlying other types of baldness in humans and may open the door to novel therapeutic approaches. Here, we show that the MU gene maps to a locus on human chromosome $8 \mathrm{p}$.

\section{Patients and Methods}

\section{Clinical Findings}

The pedigrees of families studied are shown in figure 1. Family 1 was of Dutch origin, family 2 of British origin. All kindreds examined exhibited the hallmarks of autosomal dominant inheritance, as previously described for MU. Affected persons in both families showed typical progression of alopecia, as illustrated in figure 2.

The proband in family 1 , individual VI-2, a 47-yearold white female, presented to the outpatient clinic of the Department of Clinical Genetics at the Free University Hospital in Amsterdam with complaints of progressive hair loss. Apparently, hair growth had been sparse and wiry since childhood. Eyebrows and eyelashes had always been thin. Although the hair reportedly did grow during childhood, the vertex and parietal areas remained bald. At the onset of puberty, the hair loss apparently worsened. Axillary and pubic hair failed 

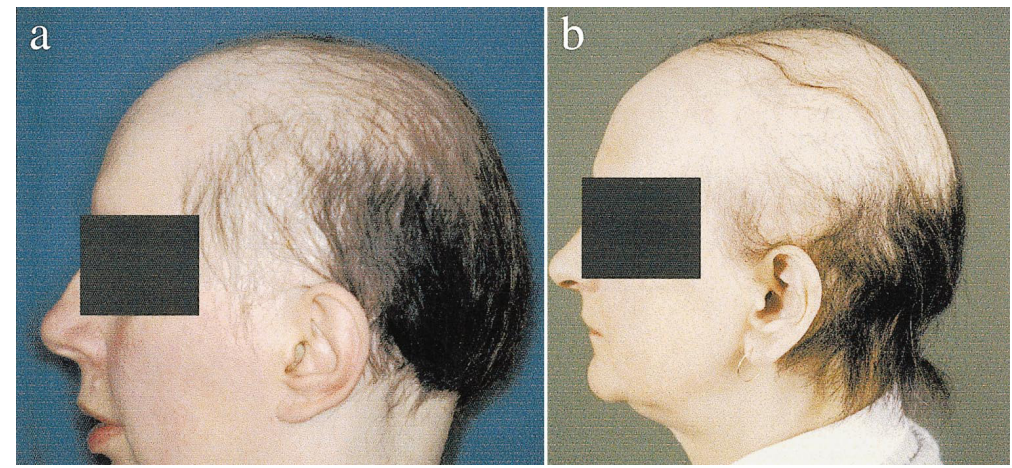

Figure 2 Clinical appearance of MU disease in similarly affected females from $(A)$ family 1 (individual VI-2) and (B) family 2 (individual IV-25). Both show the characteristic pattern of hair loss.

to develop. After the birth of her first child, the hair loss again increased. According to the patient, her mother and several other family members had an identical disorder. The same abnormalities were found in the patient's mother, whose disease history was identical. Additional affected family members were also examined and had very similar abnormalities of the hair, as well as almost identical disease histories. The pedigree was consistent with an autosomal dominant pattern of inheritance.

On examination, the patient was found to have extensive bitemporal and parieto-occipital alopecia (fig. 2). No hair-follicle openings were evident in the bald area. The remaining hair was coarse and wiry, and some hairs showed a wavy hair shaft. Eyelashes and eyebrows, as well as terminal hair on the rest of the body, was scarce. Teeth, eyes, and nails were normal. Microscopic examination of the hair showed irregular hair shafts (not shown). Knotting the hair resulted in square knots, a diagnostic feature of MU. Identical clinical and microscopic abnormalities were found in additional family members.

Family 2 was of British origin, and affected individuals presented with clinical histories essentially identical to those of the Dutch family described above. A diagnosis of MU was made. Informed consent was obtained, and blood samples for DNA analysis were collected from members of both families.

\section{Genotyping and Linkage Analysis}

Four hundred microsatellite markers were derived from the Applied Biosystems LMS2 mapping panel (Perkin-Elmer) and were used according to the manufacturer's recommended protocol with minor modifications. The main changes were that DNA was used at a concentration of $100 \mathrm{ng} / \mu \mathrm{l}$, instead of the recommended $25 \mathrm{ng} / \mu \mathrm{l}$, and that 40 PCR cycles were used. In brief, markers were PCR amplified by use of a fluorescently labeled primer and Amplitaq Gold polymerase (PerkinElmer), in buffer containing $2.5 \mathrm{mM} \mathrm{MgCl}$. The resultant PCR products were analyzed on an ABI 377 automated DNA sequencer. Gel data were extracted by use of the ABI Genescan software, and microsatellite peaks were analyzed by use of the ABI Genotyper program. For the initial genome screen, 24 meioses from family 1 were used and linkage was scored by eye. Markers that showed only one recombination event or that were either partially or completely uninformative were used to analyze 30 meioses from family 2 . Two-point LOD scores were computed by the MLINK algorithm of LINKAGE version 5.1, under the assumptions of a mutant-allele frequency of .001 and $99 \%$ penetrance. Marker-allele frequencies were assumed to be equal in the population.

\section{Mutation Detection for the $\mathrm{hr}$ Gene}

cDNA was prepared from primary epidermal keratinocyte cultures, as described elsewhere (McLean et al. 1995). The entire coding sequence of the human $b r$ gene was amplified in a series of overlapping fragments by reverse transcription-PCR (RT-PCR) with primers derived from the published cDNA sequence (Genbank accession number AF039196; Ahmad et al. 1998a). RTPCR was performed under standard conditions, and the products were directly sequenced with the ABI Prism system. Sequencing ladders were analyzed on an ABI 377 DNA sequencer. These same primers were used to amplify genomic DNA templates, by the Boehringer High Fidelity PCR system. The 3 UTR sequence was obtained by $3^{\prime}$ rapid amplification of cDNA ends (RACE) PCR with the Clontech Marathon kit. The 5' UTR sequence 
Table 1

Combined LOD Scores for MU Families 1 and 2, for 8p Markers

\begin{tabular}{lrrrrrrr}
\hline & \multicolumn{7}{c}{ LOD SCORE AT $\theta=$} \\
\cline { 2 - 8 } MARKER & \multicolumn{1}{c}{0} & \multicolumn{1}{c}{.01} & \multicolumn{1}{c}{.05} & \multicolumn{1}{c}{.10} & .20 & .30 & .40 \\
\hline D8S511 & -27.39 & -2.37 & 1.49 & 2.53 & 2.50 & 1.66 & .72 \\
D8S1827 & -18.65 & -1.33 & 1.77 & 2.67 & 2.66 & 1.89 & .91 \\
D8S549 & -11.21 & -.78 & 1.25 & 1.73 & 1.56 & 1.03 & .48 \\
D8S1731 & -27.48 & -1.68 & 2.59 & 3.78 & 3.76 & 2.69 & 1.31 \\
D8S261 & 7.53 & 7.45 & 7.03 & 6.37 & 4.81 & 3.11 & 1.44 \\
D8S1715 & 3.53 & 3.46 & 3.15 & 2.75 & 1.96 & 1.20 & .54 \\
D8S258 & 3.33 & 8.13 & 7.99 & 7.23 & 5.35 & 3.33 & 1.43 \\
D8S282 & 11.91 & 11.71 & 10.84 & 9.69 & 7.19 & 4.51 & 1.80 \\
D8S560 & 13.42 & 13.18 & 12.18 & 10.90 & 8.18 & 5.28 & 2.33 \\
D8S298 & 6.59 & 10.89 & 10.61 & 9.65 & 7.30 & 4.70 & 2.08 \\
D8S1786 & 10.48 & 10.28 & 9.46 & 8.42 & 6.27 & 4.07 & 1.92 \\
D8S1733 & 9.25 & 9.08 & 9.37 & 7.47 & 5.61 & 3.68 & 1.69 \\
D8S1752 & 10.96 & 10.78 & 10.04 & 9.03 & 6.84 & 4.50 & 2.12 \\
D8S1739 & -4.60 & 2.28 & 2.65 & 2.80 & 1.86 & 1.12 & .48 \\
D8S1771 & -6.86 & 3.30 & 4.16 & 4.04 & 3.08 & 1.87 & .74 \\
\hline
\end{tabular}

a Showed no visible recombinants.

of cDNA and the genomic equivalent, which includes an intron equivalent to intron 1 in the murine sequence, was obtained by a combination of $5^{\prime}$ RACE and crossspecies PCR with primers designed from the murine $5^{\prime}$ UTR sequence. Mutation detection was performed on an affected individual from family 1 by use of both the cDNA and the genomic DNA strategies, making, in each case, a comparison with cDNA or genomic DNA from a normal, unrelated individual.

\section{Radiation-Hybrid Mapping}

Exon 3 of the $h r$ gene was amplified by use of primers located in the flanking introns, 2 and 3: HRe3.L (sense strand, $5^{\prime}$ CCC CTT CCT TCT TGC TTG TC $3^{\prime}$ ) and HRe3.R (antisense strand, 5' ATA AAG CCT ACA GAC CCC GC $3^{\prime}$ ). This sequence tagged site was used to screen the Stanford G3 radiation-hybrid panel (Research Genetics), which gave the following data vector: $h r 00$ 0000000000000000000000000000000100000100100 11000000010000000000101000000001000000 . The microsatellite markers D8S258 and D8S298 were also mapped, with the published primers and conditions. Markers were used to screen the panel in triplicate to ensure accuracy, and the resultant data vectors were mapped via the Stanford radiation-hybrid website.

\section{Results}

Mapping of the $M U$ Gene to Human Chromosome $8 p$

A genomewide search for the MU gene was undertaken by use of the ABI LMS2 mapping panel, which consists of 400 fluorescently labeled microsatellite markers spaced at intervals $<10 \mathrm{cM}$ across the genome. Initially, 24 meioses from family 1 were analyzed, and link- age was assessed by eye. Linkage was detected with markers D1S206, D4S1597, D5S400, D8S258, and D18S57. Genotyping of additional individuals from families 1 and 2 revealed that four of these linkages were false, the exception being D8S258, which is located on chromosome $8 \mathrm{p}$. With the latter marker, analysis of additional meioses in family 1 and family 2 resulted in a significant combined two-point LOD score of 3.33 at recombination fraction $(\theta) 0$, as shown in table 1 . Linkage analysis was performed on both families by use of an additional 14 microsatellites in the vicinity of D8S258, to further define the locus. Two-point LOD scores obtained by genotyping both families with these markers are outlined in table 1 . A highly significant maximum combined two-point LOD score of 13.42 at $\theta=$ 0 was obtained with marker D8S560.

Two critical recombinants were observed in individuals from the Dutch family (family 1; fig. 1), with markers D8S258 and D8S298, as shown in figure 3. These recombination events place the MU gene in the interval between these markers, representing a genetic distance of $2.4 \mathrm{cM}$, based on the current CEPH-Généthon Integrated Map.

\section{Exclusion of the hr Gene as a Candidate for MU}

The MU locus is well within the interval to which the human $h r$ gene has been mapped, mutations in which cause the recessive hair-loss disorder congenital atrichia

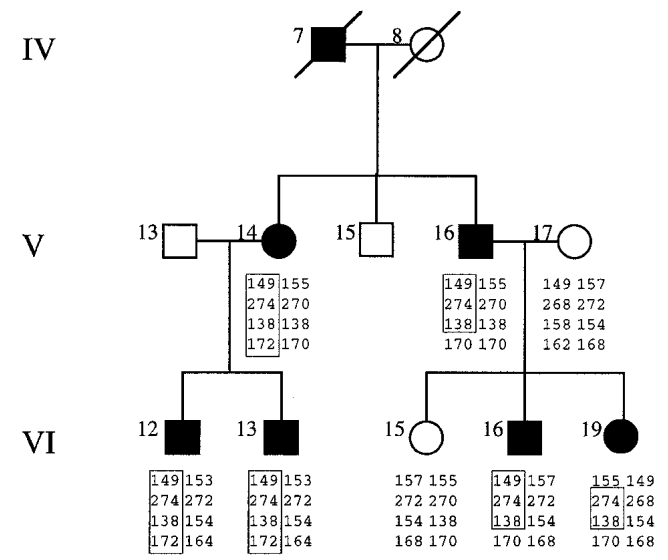

Figure 3 Haplotype analysis of part of family 1, showing the two recombinants that delineate the $2.4-\mathrm{cM}$ MU critical region between markers D8S258 (distal) and D8S298 (proximal). As shown in figure 4 , distances of markers from the telomere, according to Généthon map, are: D8S258, 40.3 cM; D8S282, 41.6 cM; D8S560, $42.2 \mathrm{cM}$; and D8S298, $42.7 \mathrm{cM}$. 
(Ahmad et al. 1998a; Cichon et al. 1998). Specifically, the previous mapping studies had placed the $h r$ gene in the interval between D8S261 and D8S1771, by use of radiation-hybrid mapping (Cichon et al. 1998). Since we had critical recombinants with D8S258 and D8S298, which are $2.4 \mathrm{cM}$ apart in the middle of this region, we performed radiation-hybrid mapping using the Stanford G3 panel to discover if the $h r$ gene lies within this MU critical region. D8S258, D8S298, and the $h r$ gene were scored in triplicate on the G3 panel. Data vectors obtained for D8S258 and D8S298 were identical to those reported by the Stanford Human Genome Center. The br gene was placed $7 \mathrm{cR}_{10,000}$ (centiRays for a 10,000 rad radiation hybrid panel) distant from D8S298 (fig. 4). However, the G3 panel was unable to resolve the order of markers at this locus. (A description of how the G3 radiation hybrid panel was constructed and an explanation of the units of distance used can be found at the Stanford radiation-hybrid website.) Therefore, we were unable to exclude the $h r$ gene from the MU interval by this means and proceeded to analyze the $h r$ gene for mutations in MU. Interestingly, the order of markers on the G3 map of this region (fig. 4) is not fully consistent with the Généthon linkage map of the locus (table 1). Specifically, the positions of D8S560 and D8S1733 are reversed on these two maps. Our linkage data are consistent with the Généthon ordering of these markers, and one possibility is that the G3 data for these markers have been switched. Physical mapping of the locus should further resolve these inconsistencies.

Mutation detection for the $h r$ gene was performed in two ways. First, the entire $h r$ cDNA was amplified by RT-PCR using mRNA derived from skin-biopsy samples from an affected individual in family 1 and a normal unrelated individual and was fully sequenced. The UTRs of the human $h r$ mRNA were not present in the GenBank entry for the gene and so were first determined by $5^{\prime}$ and $3^{\prime}$ RACE techniques. This was particularly important in the case of the $5^{\prime}$ UTR sequence, which contains an intron in mice and therefore might harbor splicing mutations (Cachon-Gonzalez et al. 1994). Sequencing of the cDNA revealed a number of minor sequence changes from the published human $b r$ cDNA sequence (Ahmad et al. 1998a), as reported (Cichon et al. 1998; Ahmad et al. 1999). All changes observed were also detected in normal unrelated individuals and were therefore excluded as pathogenic mutations. Second, mutation detection was performed by use of genomic DNA. Initially, the intron-exon organization of $h r$ was not available, and so we determined it independently, although these data have been recently reported by other groups $(\mathrm{Ci}-$ chon et al. 1998; Ahmad et al. 1999). We found the intron-exon organization of the gene to be identical to that published. We also cloned the $5^{\prime}$ UTR sequence from cDNA and genomic DNA by a combination of $5^{\prime}$ RACE

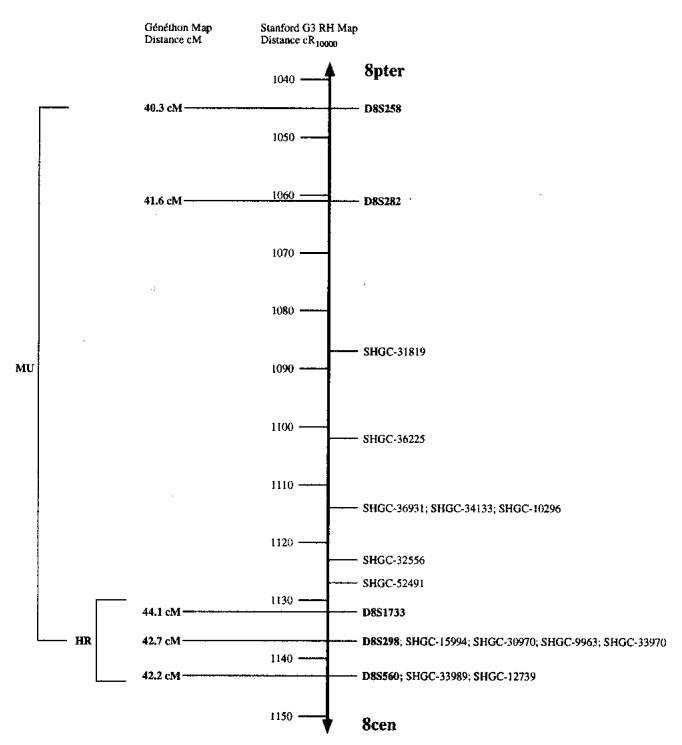

Figure 4 Radiation-hybrid map of MU locus, based on the Stanford $\mathrm{G} 3$ panel. Distances are in $\mathrm{CR}_{10,000}$. On the basis of this mapping panel, the $h r$ gene was located $7 \mathrm{cR}_{10,000}$ from marker D8S298 but could not be ordered relative to this and nearby markers. Note that the order of markers is different from that given by the Généthon linkage map (see table 1): D8S560 and D8S1733 are in reverse order. Our linkage data are consistent with the Généthon order. Physical mapping of the locus should resolve this inconsistency.

PCR and cross-species PCR, using primers derived from the murine sequence. Like the murine sequence, the human $5^{\prime}$ UTR of the $h r$ gene contains an intron (Cichon et al. 1998; Ahmad et al. 1999). Again, no mutations at the genomic DNA level, including all intronic splicing and branch point sites, were found in an affected person from family 1 .

\section{Discussion}

Here, by genomewide linkage analysis with fluorescent microsatellite markers, we have mapped an autosomal dominant gene for a human hereditary hair-loss syndrome, MU. On the basis of two critical recombination events in the Dutch family studied (family 1; fig. 1 ), we have shown that the gene for MU is located in a 2.4-cM region between Généthon markers D8S258 (distal) and D8S298 (proximal) on human chromosome 8p22-21 (fig. 3). A strong candidate gene in this region is the human homologue of the murine hairless gene, $h r$, which was previously mapped to the center of a region delineated by markers D8S261 and D8S1771 (Ahmad et al. 1998a). Homozygous mutations in this gene have 
been demonstrated in families with the autosomal recessive disorder congenital atrichia. These include homozygous missense mutations (Ahmad et al. 1998a, 1998b; Cichon et al. 1998); homozygous deletion mutations (Zlotogorski et al. 1998; Ahmad et al. 1999); and a homozygous splice-donor mutation (Cichon et al. 1998). Homozygous loss-of-function mutations in the murine $h r$ gene have recently been shown to underlie various rhino mouse phenotypes: $h r^{\text {rh-8J }}$ (Ahmad et al. $1998 d) ; r^{\text {rhY }}$ (Panteleyev et al. 1998a); and $h r^{\text {rhChr }}($ Ahmad et al. 1998c), in addition to the original hairless phenotype (Cachon-Gonzalez et al. 1994). The $h r$ polypeptide is a putative transcription factor that may control apoptotic events in the hair cycle (Panteleyev et al. $1998 b$ ). Here, we have performed higher-resolution radiation-hybrid mapping of $h r$ but have been unable to exclude it from the MU critical region. We have shown by radiation-hybrid mapping that $h r$ lies very close to D8S298, a marker with which we observed recombination in MU (fig. 4). However, we were not able to place this gene outside the MU locus by this method.

Autosomal dominant inheritance has not been described for congenital atrichia, and the heterozygous carriers of the mutant alleles reported are apparently asymptomatic (Ahmad et al. 1993, 1998a, 1998b; Cichon et al. 1998; Zlotogorski et al. 1998). We speculated that MU might be caused by dominant-negative mutations in $h r$, whereas the mutations seen in congenital atrichia act in a recessive, loss-of-function fashion. Support for this hypothesis was gained from examination of the Mouse Genome Database. There are several independent mutant alleles of the $h r$ gene in mice, such as the rhino alleles $h r^{\text {rh }}$ (Howard 1940), $h r^{\text {rh }}-8 J$ (Ahmad et al. 1998d); $h r^{\text {rhY }}$ (Panteleyev et al. 1998a), and $h r^{\text {rhChr }}$ (Ahmad et al. 1998c), the bald allele $h r^{\text {ba }}$ (Garber 1952); and the insertional mutant $h r^{\mathrm{TgN} N 5033 \mathrm{Mm}}$ (Jones et al. 1993). All of these alleles are recessive; however, one mouse mutant known as "near-naked," $b r^{n}$, is allelic with the recessive $h r$ mutations and shows semidominance, giving a milder hair-loss phenotype in heterozygotes and a more severe phenotype in homozygotes (Stelzner 1983). This evidence, combined with the close proximity of the human $h r$ gene to the MU locus, led us to postulate that MU might well be a dominantly acting mutation in $h r$, and so we undertook mutation detection for this gene in our MU patients, using both cDNA and genomic DNA. However, no mutations were found by either approach.

In conclusion, the MU gene maps to a locus close to but apparently distinct from the $h r$ gene on $8 \mathrm{p} 22-21$. There are several expressed sequence tags that map to this region, none of which represent good candidates and the vast majority of which are anonymous (NCBI Gene Map '98). Extensive BLAST analysis of these sequences (Altschul et al. 1990; Altschul et al. 1997) failed to identify any of them with homology to $h r$, to other transcription factors, or to other potential candidates. We are now constructing a physical map of the locus to allow identification of the MU gene, a gene that undoubtedly encodes a protein that plays an important role in hair development and maintenance in humans.

\section{Acknowledgments}

We thank the patients and their families for making this study possible and thank Hans-Jürg Alder and his staff in the Nucleic Acid Facility, Kimmel Cancer Center, Jefferson Medical College, Philadelphia, for DNA synthesis and sequencing. This work was supported by grants from the Dystrophic Epidermolysis Bullosa Research Associations of the U.K. and of America; and by U.S. Public Health Service, National Institutes of Health grant PO1-AR38923. W.H.I.M. is supported by a Wellcome Trust Senior Research Fellowship. M.V.S. is supported by Netherlands Organization of Scientific Research grant 920-03-085, a grant from Merck Sharp and Dohme BV, and the Dutch Skin Foundation.

\section{Electronic-Database Information}

Accession numbers and URLs for data in this article are as follows:

CEPH-Généthon Integrated Map, http://www.cephb.fr/cephgenethon-map.html

GenBank Entrez Browser, http://www.ncbi.nlm.nih.gov/ Entrez/nucleotide.html

Mouse Genome Database, http://www.informatics.jax.org/ NCBI Gene Map '98, http://www.ncbi.nlm.nih.gov/

Online Mendelian Inheritance in Man (OMIM), http://www .ncbi.nlm.nih.gov/Omim (for MU and congenital atrichia with papular lesions)

Stanford radiation-hybrid website, http://www-shgc.stanford .edu/RH/index.html

\section{References}

Ahmad M, Abbas H, Haque S (1993) Alopecia universalis as a single abnormality in an inbred Pakistani kindred. Am J Med Genet 46:369-371

Ahmad W, Haque MF, Brancolini V, Tsou HC, Haque S, Lam H, Aita VM, et al (1998a) Alopecia universalis associated with a mutation in the human hairless gene. Science 279: 720-724

Ahmad W, Irvine AD, Lam H, Buckley C, Bingham EA, Panteleyev AA, Ahmad M, et al (1998b) A missense mutation in the zinc-finger domain of the human hairless gene underlies congenital atrichia in a family of Irish Travellers. Am J Hum Genet 63:984-991

Ahmad W, Panteleyev AA, Henson-Apollonio V, Sundberg JP, Christiano AM (1998c) Molecular basis of a novel rhino $\left(h r^{\text {rhChr }}\right)$ phenotype: a nonsense mutation in the mouse hairless gene. Exp Dermatol 7:298-301

Ahmad W, Panteleyev AA, Sundberg JP, Christiano AM $(1998 d)$ Molecular basis for the rhino $\left(h^{r \text { th-8J }}\right)$ phenotype: a 
nonsense mutation in the mouse hairless gene. Genomics 53: 383-386

Ahmad W, Zlotogorski A, Panteleyev AA, Lam H, Ahmad M, ul Haque MF, Abdallah HM, et al (1999) Genomic organization of the human hairless gene (HR) and identification of a mutation underlying congenital atrichia in an Arab Palestinian family. Genomics 56:141-148

Altschul SF, Gish W, Miller W, Myers EW, Lipman DJ (1990) Basic local alignment search tool. J Mol Biol 215:403-410

Altschul SF, Madden TL, Schaffer AA, Zhang J, Zhang Z, Miller W, Lipman DJ (1997) Gapped BLAST and PSIBLAST: a new generation of protein database search programs. Nucleic Acids Res 25:3389-3402

Bentley-Phillips B, Grace HJ (1979) Hereditary hypotrichosis: a previously undescribed syndrome. Br J Dermatol 101: 331-339

Borelli S (1954) Hypotrichosis congenita hereditaria Marie Unna. Der Hautarzt 5:18-22

Brooke HC (1926) Hairless mice. J Hered 17:173-174

Cachon-Gonzalez MB, Fenner S, Coffin JM, Moran C, Best S, Stoye JP (1994) Structure and expression of the hairless gene of mice. Proc Natl Acad Sci USA 91:7717-7721

Cichon S, Anker M, Vogt IR, Rohleder H, Putzstuck M, Hillmer A, Farooq SA, et al (1998) Cloning, genomic organization, alternative transcripts and mutational analysis of the gene responsible for autosomal recessive universal congenital alopecia. Hum Mol Genet 7:1671-1679

Dawber R (1997) Diseases of the hair and scalp. Blackwell Scientific, Oxford

Garber ED (1952) Bald, a second allele of hairless in the house mouse. J Hered 43:45-46

Healy E, Holmes SC, Belgade C, Stephenson AM, McLean WHI, Rees JL, Munro CS (1995) A gene for monilethrix is closely linked to the keratin gene cluster on chromosome 12q. Hum Mol Genet 4:2399-2402

Howard A (1940) "Rhino," an allele of hairless in the house mouse. J Hered 31:467-470

Jones JM, Elder JT, Simin K, Keller SA, Meisler MH (1993) Insertional mutation of the hairless locus on mouse chromosome 14. Mamm Genome 4:639-643

Kere J, Srivastava AK, Montonen O, Zonana J, Thomas N, Ferguson B, Munoz F, et al (1996) X-linked anhidrotic (hypohidrotic) ectodermal dysplasia is caused by mutation in a novel transmembrane protein. Nat Genet 13:409-416

Kibar Z, Der Kaloustian VM, Brais B, Hani V, Fraser FC, Rouleau GA (1996) The gene responsible for Clouston hidrotic ectodermal dysplasia maps to the pericentromeric region of chromosome 13q. Hum Mol Genet 5:543-547

Laass MW, Hennies HC, Preis S, Stevens HP, Jung M, Leigh IM, Wienker TF, et al (1997) Localisation of a gene for Papillon-Lefevre syndrome to chromosome 11q14-q21 by homozygosity mapping. Hum Genet 101:376-382
Ludwig E (1953) Hypotrichosis congenita hereditaria typ Marie Unna. Arch Dermatol Syphilis 196:261-278

McGrath JA, McMillan JR, Shemanko CS, Runswick SK, Leigh IM, Lane EB, Garrod DR, et al (1997) Mutations in the plakophilin 1 gene can result in ectodermal dysplasia/ skin fragility syndrome. Nat Genet 17:240-244

McLean WHI, Rugg EL, Lunny DP, Morley SM, Lane EB, Swensson O, Dopping-Hepenstal PJC, et al (1995) Keratin 16 and keratin 17 mutations cause pachyonychia congenita. Nat Genet 9:273-278

Nothen MN, Cichon S, Vogt IR, Hemmer S, Kruse R, Knapp M, Holler T, et al (1998) A gene for universal congenital alopecia maps to chromosome 8p21-22. Am J Hum Genet 62:386-390

Panteleyev AA, Ahmad W, Malashenko AM, Ignatieva EL, Paus R, Sundberg JP, Christiano AM (1998a) Molecular basis of the rhino Yurlovo $\left(h r^{\text {rhY }}\right)$ phenotype: severe skin abnormalities and female reproductive defects associated with an insertion in the hairless gene. Exp Dermatol 7: 281-288

Panteleyev AA, Paus R, Ahmad W, Sundberg JP, Christiano AM (1998b) Molecular and functional aspects of the hairless (hr) gene in laboratory rodents and humans. Exp Dermatol 7:249-267

Peachey RD, Wells RS (1971) Hereditary hypotrichosis (Marie Unna type). Trans St Johns Hosp Dermatol Soc 57:157-166

Solomon LM, Esterly NB, Medenica M (1971) Hereditary trichodysplasia: Marie Unna's hypotrichosis. J Invest Dermatol 57:389-400

Speigel B, Hundeiker M (1979) Hypotrichosis congenita hereditaria. Fortschr Med 97:2018-2022

Stelzner KF (1983) Four dominant autosomal mutations affecting skin and hair development in the mouse. J Hered 74: 193-196

Stevanovic DV (1970) Hereditary hypotrichosis congenita: Marie Unna type. Br J Dermatol 83:331-337

Unna M (1925) Ueber Hypotrichosis congenita hereditäria. Dermatol Wochenschr 81:1167-1178

Winter H, Rogers MA, Gebhardt M, Wollina U, Boxall L, Chitayat D, Babul-Hirji R, et al (1997a) A new mutation in the type II hair cortex keratin hHb1 involved in the inherited hair disorder monilethrix. Hum Genet 101:165-169

Winter H, Rogers MA, Langbein L, Stevens HP, Leigh IM, Labreze C, Roul S, et al (1997b) Mutations in the hair cortex keratin hHb6 cause the inherited hair disease monilethrix. Nat Genet 16:372-374

Wirth G, Bindewald J, Kuester W, Goerz G (1985) Hypotrichosis congenita hereditäria Marie Unna. Hautarzt 36: 577-580

Zlotogorski A, Ahmad W, Christiano AM (1998) Congenital atrichia in five Arab Palestinian families resulting from a deletion mutation in the human hairless gene. Hum Genet 103:400-404 
Chapter 3 


\section{A Novel Connexin 26 Mutation in a Patient Diagnosed with Keratitis-Ichthyosis-Deafness Syndrome}

Maurice A. M. van Steensel, ${ }^{1}$ Michel van Geel, ${ }^{1}$ Marc Nahuys, ${ }^{*}$ J. Henk Sillevis Smitt, ${ }^{*}$ and Peter M. Steijlen Department of Dermatology, University Medical Center Nijmegen, the Netherlands; *Department of Dermatology, Academic Medical Center Amsterdam, University of Amsterdam, the Netherlands

Keratitis-ichthyosis-deafness syndrome is a rare disorder characterized by erythrokeratoderma, deafness, and keratitis. Scarring alopecia and squamous cell carcinoma can also occur. Most cases described so far were sporadic. Here we present evidence that keratitis-ichthyosis-deafness syndrome is caused by a mutation in the connexin 26 gene. This finding expands the spectrum of disorders caused by defects in connexin 26 and implies the gene in normal corneal function, hair growth, and carcinogenesis. Key words: skin cancer/alopecia/gap junction. J Invest Dermatol 118:724-727, 2002
$\mathrm{K}$

eratitis-ichthyosis-deafness (KID) syndrome is a rare autosomal dominant disorder. It is characterized by the occurrence of localized erythematous scaly skin lesions, severe bilateral keratitis, and sensorineural deafness (Rycroft et al, 1976; Cram et al, 1979; Skinner et al, 1981; Singh, 1987; Langer et al, 1990; McGrae, 1990; Morris et al, 1991; Nurse, 1994; Caceres-Rios et al, 1996; Alli and Gungor, 1997; Kone-Paut et al, 1998). The term "ichthyosis" is, strictly speaking, not correct, as the skin lesions are more appropriately classified as erythrokeratoderma.

A scarring alopecia can be part of the phenotype. The skin lesions occur predominantly on the face, palms, and soles, and have a typical reticulated pattern that is often called leather-like. Squamous cell carcinoma has been reported in $11 \%$ of the patients and may probably be considered as a manifestation of the disease (Grob et al, 1987; Madariaga et al, 1986; Hazen et al, 1989, 1992; Morris et al, 1991). Histologic examination usually shows nonspecific changes but may show severe follicular plugging.

The combination of erythrokeratoderma and deafness also occurs in erythrokeratoderma variabilis of Mendes da Costa, an autosomal dominant disorder that has been shown to be caused by mutations in the connexin (CX) genes 30.3 and 31 (Richard et al, 1998; Wilgoss et al, 1999; Kelsell et al, 2000). Although keratitis is not part of erythrokeratoderma variabilis, the skin lesions and sensorineural deafness are similar to those found in KID syndrome. Therefore, we considered the connexin genes that are expressed in skin excellent candidates for KID syndrome.

We ascertained a patient suffering from KID syndrome. She is the only affected person in the family (Cremers et al, 1977). The patient, the youngest of nine children, was born at term from consanguineous (third degree) Dutch parents. Pregnancy was uneventful. During the first weeks after birth, thickening and scaling of the skin became apparent, as well as a reddish-brown discoloration of affected skin. The patient reportedly had trouble sweating. At $4 \mathrm{y}$ of age, the parents first noted hearing loss.

Manuscript received November 20, 2001; revised December 12, 2001; accepted for publication December 20, 2001

Reprint requests to: Dr. M.A.M. van Steensel, Department of Dermatology, University Medical Center Nijmegen, PO Box 9101, 6500 HB Nijmegen, the Netherlands. Email: m.vansteensel@derma.azn.nl

${ }^{1}$ Both authors contributed equally to this work.
Psychomotor development was normal. From $11 \mathrm{y}$ of age, the patient developed bilateral keratitis with photophobia. Repeated keratoconjunctivitis with superficial and deep neovascularization of both lenses necessitated the implantation of artificial lenses at age 34. This intervention in turn induced a bullous corneal dystrophy. At $38 \mathrm{y}$ of age, she developed a skin lesion on the right ankle that was initially diagnosed as pseudo-epitheliomatous hyperplasia. Later, the diagnosis was revised as spinocellular carcinoma. The lesion was excised and the patient remains free of disease to date.

Physical examination at age 18 showed red, hyperkeratotic skin on much of the body surface. The nails of hands and feet were thickened. Scalp hair was brittle, eyebrows and eyelashes were sparse, whereas pubic and axillary hair were missing altogether. Mammary gland development was insufficient for age (Fig 1). Dentition was abnormal; the teeth were small and abnormally shaped. Ophthalmologic examination showed bilateral bullous corneal dystrophy with neovascularization. Bilateral astigmatism was noted as well. Audiologic examination demonstrated profound bilateral sensorineural hearing loss. No other abnormalities were noted and a karyotype was a normal 46,XX.

Blood was taken from the patient, her mother, and four sibs, and DNA extracted from peripheral blood leukocytes using methods described elsewhere (Miller et al, 1988). The father was deceased. We sequenced connexin genes that are known or expected to be involved in skin disorders and sometimes accompanied by deafness. The genes that were analyzed are CX26 (GJB2), 30 (GJB6), 30.3 (GJB3), 31 (GJB5), 31.1 (GJB4), and 37 (GJA4). We did not sequence CX43. It is expressed in skin (Goliger and Paul, 1994), but has been implicated mainly in cardiac morphogenesis and function (Huang et al, 1998) and lens function (Gao and Spray, 1998).

Primer sequences were as follows: Cx26F, GCATGCTTGCTTACCCAGACTC; Cx26R, AGGGGAGCAGAGCTCCATTG; Cx30F, AGCAGGGCAGGGAGTTGAAG; Cx30R, TCAGGTTGGTATTGCCTTCTGG; Cx30.3F, CAATCGCACCAGCATTAAGGG; Cx30.3R, TGATCTTATCTGCTGATCTCGCAG; Cx31F, TTCATTCATACGATGGTTTTTCCTC; Cx31R, ACCTCTCCACCTGCCACACC; Cx31.1F, GAACCCAGCTCCTCTAGTGATGG; Cx31.1R, CCATCCAGGCCCAACCTG. The sequences were assembled and analyzed using the Phred-Phrap-Consed software tools (Ewing and Green, 


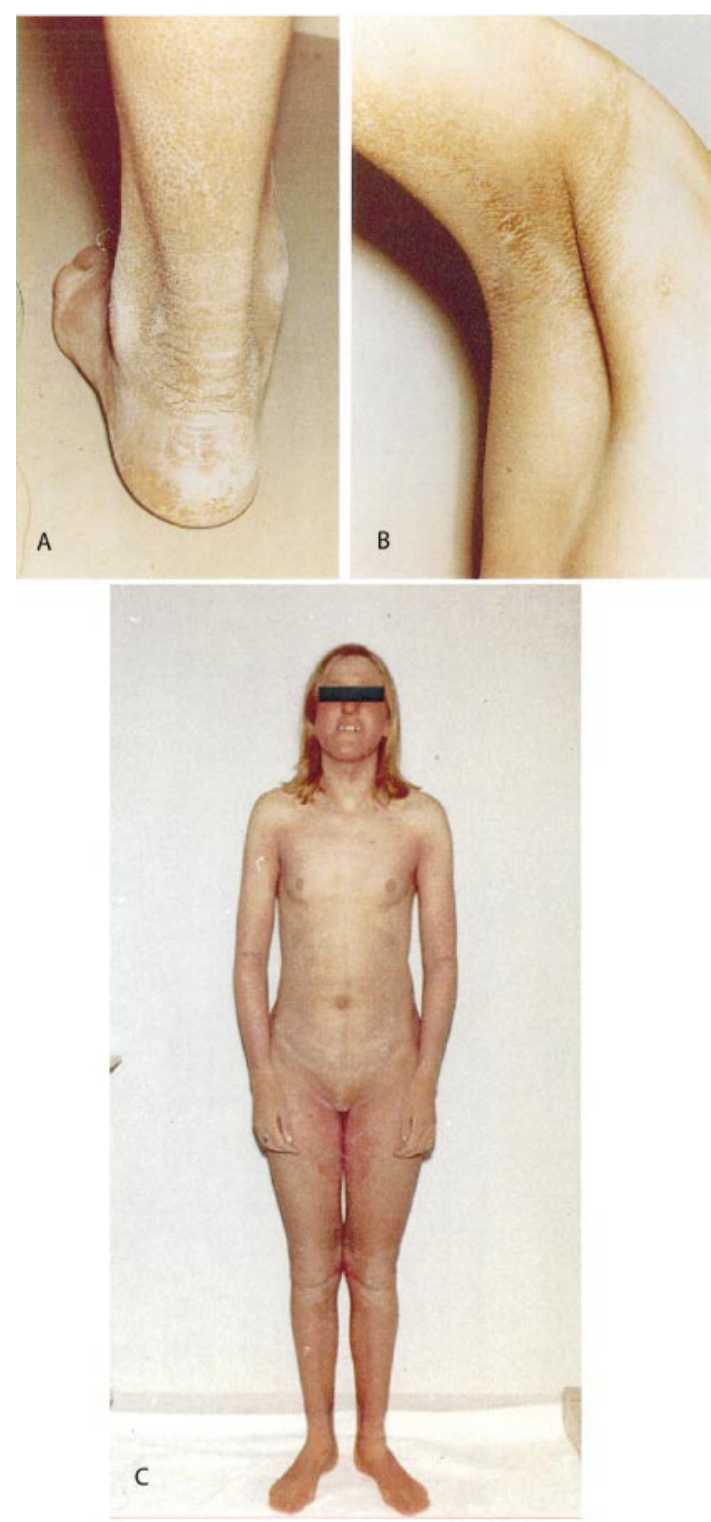

Figure 1. Phenotype of the patient. (A) Typical shark-skin-like hyperkeratosis and erythroderma on left calf and ankle. (B) Hypotrichosis and hyperkeratosis in right axilla $(C)$ Frontal view of patient. Mask-like and hyperkeratosis in right axilla. (C) Frontal view of patient. Mask-like erythrokeratoderma of the face, lack of pubic hair, pronounced erythroderma of extremities with sparing of rump. Vestigial mammary
glands.

1998; Ewing et al, 1998; Gordon et al, 1998). No mutations were found in CX30, CX30.3, CX31, and CX31.1. In CX26 the patient had a heterozygous GAC to AAC change in codon 50. This changes a conserved aspartic acid into an asparagine in the first extracellular domain (D50N). Because the $\mathrm{G}$ to A change abolishes an AspI (Roche Diagnostics, DE-68305 Mannheim, Germany) restriction site, we examined controls and the family by restriction analysis. The mutation was not present in 164 control alleles and could not be demonstrated in the mother and four sibs either (Fig 2). The absence in 164 control alleles and the other family members strongly suggests that it is not a polymorphism; however, recent evidence suggests that some disorders associated with connexin mutations can be digenic. Kelsell et al (2000) have demonstrated that a variation in CX26 (M34T) can interact with mutations in CX26 and CX31 to produce a more severe hearing loss than occurs in single CX26/31 mutants. It is possible that a similar phenomenon is at work in KID syndrome, explaining its rarity and the relative lack of instances with autosomal dominant inheritance. We did not find mutations in CX30, CX30.3, CX31, CX31.1, and CX37 or in the other CX26 allele, suggesting that in this particular case a digenic mutation is less likely. The finding of this novel mutation expands the spectrum of disorders in which CX26 is involved. So far, it has been implicated in a variant of Vohwinkel's syndrome (MIM 124500), palmoplantar keratoderma-deafness syndrome and nonsyndromic hearing loss.

The aspartic acid at position 50 is conserved across species and across the connexins suggesting that it is of vital importance for correct functioning of the protein (Fig 3). It has been demonstrated that a substitution in CX26 (W77R) impairs transport of small charged molecules across gap junctions (White, 2000). This mutation also leads to inefficient targeting of the protein product to the plasma membrane with subsequent retention in intracellular stores. In addition, the mutated connexin showed limited oligomerization into connexon hemichannels. It is tempting to speculate that the KID syndrome mutation has a similar effect; however, the W77R mutation is recessive, suggesting that the KID mutation must have additional effects.

As the D50N change replaces a charged amino acid by an uncharged one, the substitution can be expected to affect local conformation. It may also influence voltage gating. Recent data suggest that single gene mutations may affect voltage-dependent gating in heterotypic channels such as those formed by CX26 and CX32 (Zhao and Santos-Sacchi, 2000). In addition, the introduction of charge at the start of the first extracellular loop can be expected to disturb local conformation and thus interfere with docking to the partner connexin. It has been demonstrated that local E1 topology is essential for connexon formation (Foote et al, 1998). The clustering of skin disease associated CX26 mutations in this domain suggests that this domain is of special importance in skin, either for skin-specific connexon assembly or for interactions with other proteins. In other connexins, the mutations causing erythrokeratoderma variabilis are clustered in the first transmembrane domain, supporting the hypothesis that the CX26 E1 domain has a special function in skin physiology. This issue needs to be addressed in future studies.

Of interest is the role for CX26 in the cornea that is suggested by our findings. The main gap junction protein in the cornea seems to be CX43 (Nishida et al, 1996). No CX26 expression has so far been found in corneal epithelium from many animal species (White and Bruzzone, 2000); however, human cornea has to our knowledge not yet been examined for CX26 expression.

Homozygous CX43 knockout mice have lens abnormalities consisting of separation and vacuolization of lens fibrils, interpreted as early signs of cataract (Gao and Spray, 1998). Apparently, this connexin is required for maintenance of osmotic pressure in the lens. It is tempting to speculate that CX26 has a similar role in the human cornea. If corneal keratinocytes were to become separated, infectious agents might be able to establish a presence in between the corneocytes. This would lead to keratitis. Other disorders caused by CX26 mutations are not accompanied by overt corneal disease. Skin symptoms, however, are associated with particular mutations and it is conceivable that the same applies to corneal involvement in which case the communication or osmotic pressure hypotheses would not be tenable as sole explanation. It would be of considerable interest to examine other forms of corneal dystrophy for connexin mutations in order to test this assertion. 
A
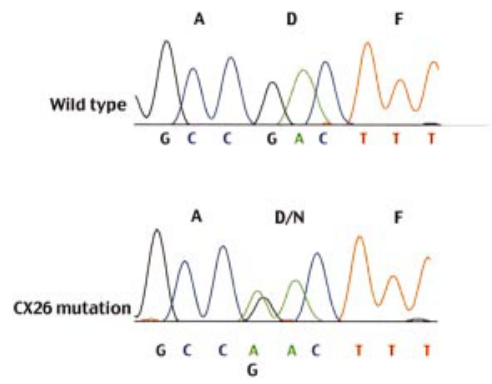

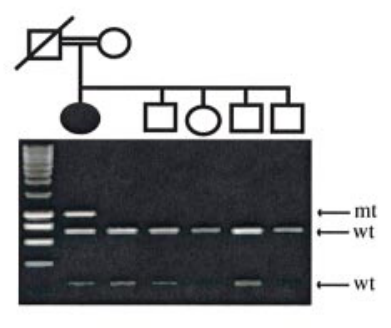

Figure 2. Mutation analysis. (A) Sequence traces of wild-type sequence $v s$ patient sequence. $\mathrm{G} \rightarrow \mathrm{A}$ transversion changing codon 50 from GAC to AAC. (B) The mutation abolishes an AspI restriction site. Restriction analysis demonstrates the presence of a mutated allele in the proposita only.

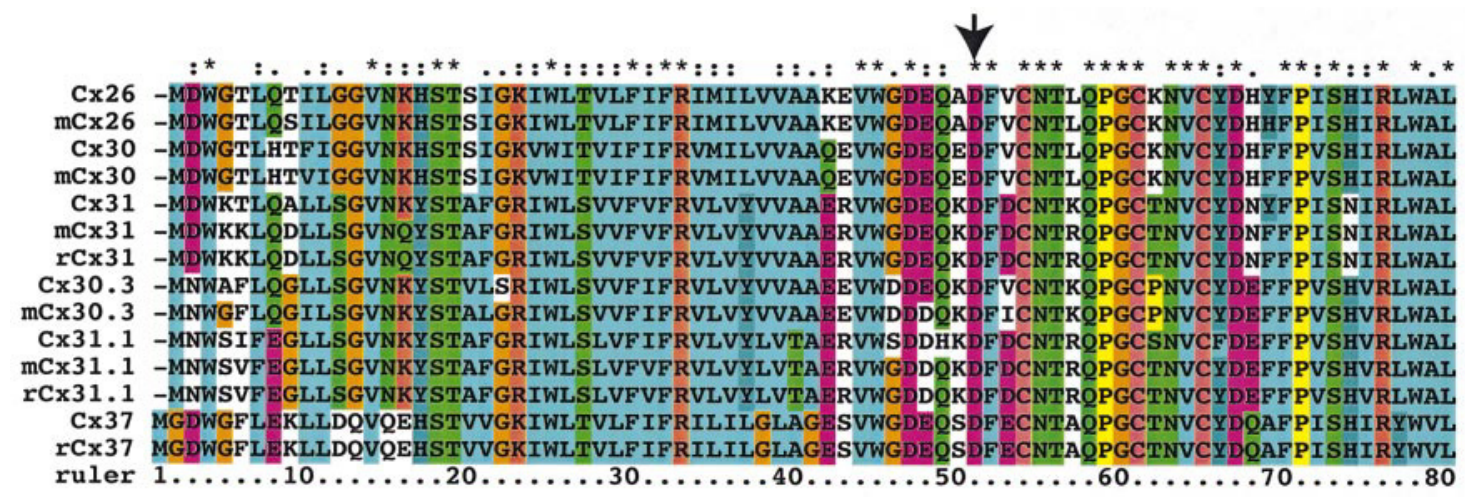

Figure 3. CLUSTALX alignment of connexin proteins from human, mouse, and rat. D50 is conserved in all connexins in the alignment (arrow).

The scarring alopecia observed in KID syndrome is probably related to the follicular plugging that is commonly observed. A role for CX26 in hair follicle differentiation is suggested by the hypotrichosis observed elsewhere on the body. Thus far, only CX30 has been implicated in hair growth. This aspect of the phenotype is likely related to the specific mutation we observe here, as other disorders caused by CX26 mutations are not characterized by hypotrichosis. Thus, as in the case of the keratitis, it is not likely that the hypotrichosis is related solely to a disturbance of intercellular communication. The same can be said for the propensity for developing squamous cell carcinomas, which is observed in KID syndrome but not in other disorders caused by connexin mutations. CX26 is known to be reduced or absent in mammary carcinoma cells and is considered a putative tumor suppressor for epithelial tumors (Lee et al, 1991, 1992; Tu et al, 1998; Singal et al, 2000). Other connexins such as CX37 have been shown to be involved in tumorigenesis. Specifically, CX37 mutations have been described in vinyl chloride induced hepatic angiosarcomas (Saito et al, 1997) and disturbed gap junction communication has been reported in many other tumor types. CX32 mutant mice are prone to liver cancer (Moennikes et al, 2000). No definite connexin mutations have been reported in human cancers or cancer-prone disorders. Our findings are the first to suggest that germline connexin mutations can lead to skin cancer in humans.

CX26 is known to upregulate E-cadherin expression (Stoler et al, 1993). As E-cadherin is probably involved in the regulation of hair growth (Van Steensel et al, 2000, 2001) and is downregulated in approximately $70 \%$ of squamous cell carcinomas examined in one study (Koseki et al, 1999) it is likely that alterations of E-cadherin expression are involved in the increased cancer susceptibility and hypotrichosis of KID syndrome.

In conclusion, the finding of a novel CX26 mutation in KID syndrome supports the notion that connexins have functions not directly related to their presence in gap junctions and demonstrates that germ-line connexin mutations can cause cancer in humans. It appears that deafness and erythroderma are symptoms that may be related to disturbed gap junction function per se. Other symptoms such as the keratitis and the cancer-proneness seem to be dependent upon mutations in a particular residue suggesting that disturbance of gap junction formation is not sufficient as an explanation and that there may be direct interactions with the cytoskeleton or cell-cycle machinery dependent upon specific amino acid motifs in the connexin protein.

M.v.G. is supported by a grant from Rebirth SA, Luxembourg. M.v.S. is M.v.G. is supported by a grant from Rebirth $S A$, Luxembourg. M.v.S. is
supported by grants from Rebirth $S A$, Luxembourg and the Dutch Organization for Research NWO grant number 920-03-085.

\section{REFERENCES}

Alli N, Gungor E: Keratitis, ichthyosis and deafness (KID) syndrome. Int J Dermatol 36:37-40, 1997

Caceres-Rios H, Tamayo-Sanchez L, Duran-Mckinster C, de la Luz Orozco M, Ruiz-Maldonado R: Keratitis, ichthyosis, and deafness (KID syndrome): 
review of the literature and proposal of a new terminology. Pediatr Dermatol 13:105-113, 1996

Cram DL, Resneck JS, Jackson WB: A congenital ichthyosiform syndrome with deafness and keratitis. Arch Dermatol 115:467-471, 1979

Cremers CW, Philipsen VM, Mali JW: Deafness, ichthyosiform erythroderma, corneal involvement, photophobia and dental dysplasia.J Laryngol Otol 91:585590,1977

Ewing B, Green P: Base-calling of automated sequencer traces using phred. II. Error probabilities. Genome Res 8:186-194, 1998

Ewing B, Hillier L, Wendl MC, Green P: Base-calling of automated sequencer traces using phred. I. Accuracy assessment. Genome Res 8:175-185, 1998

Foote CI, Zhou L, Zhu X, Nicholson BJ: The pattern of disulfide linkages in the extracellular loop regions of connexin 32 suggests a model for the dockins

Gao Y, Spray DC: Structural changes in lenses of mice lacking the gap junction protein connexin43. Invest Ophthalmol Vis Sci 39:1198-1209, 1998

Goliger JA, Paul DL: Expression of gap junction proteins Cx26, Cx31.1, Cx37, and Cx43 in developing and mature rat epidermis. Dev Dyn 200:1-13, 1994

Gordon D, Abajian C, Green P: Consed: a graphical tool for sequence finishing.

Genome Res 8:195-202, 1998
Grob JJ, Breton A, Bonafe JL, Sauvan-Ferdani M, Bonerandi JJ: Keratitis, ichthyosis, and deafness (KID) syndrome. Vertical transmission and death from multiple and deafness (KID) syndrome. Vertical transmission and deat
squamous cell carcinomas. Arch Dermatol 123:777-782, 1987

Hazen PG, Carney P, Lynch WS: Keratitis, ichthyosis, and deafness syndrome with development of multiple cutaneous neoplasms. Int J Dermatol 28:190-191, 1989

Hazen PG, Walker AE, Stewart JJ, Carney JF, Engstrom CW, Turgeon KL: Keratiti, ichthyosis, and deafness (KID) syndrome: management with chronic oral ketoconazole therapy. Int J Dermatol 31:58-59, 1992

Huang GY, Wessels A, Smith BR, Linask KK, Ewart JL, Lo CW: Alteration in connexin 43 gap junction gene dosage impairs conotruncal heart development. Dev Biol 198:32-44, 1998

Kelsell DP, Wilgoss AL, Richard G, Stevens HP, Munro CS, Leigh IM: Connexin mutations associated with palmoplantar keratoderma and profound deafness in single family. Eur J Hum Genet 8:469-472, 2000

Kone-Paut I, Hesse S, Palix C, Rey R, Remediani K, Garnier JM, Berbis P. Keratitis, ichthyosis, and deafness (KID) syndrome in half sibs. Pediatr Dermato 15:219-221, 1998

Koseki S, Aoki T, Ansai S, Hozumi Y, Mitsuhashi Y, Kondo S: An immunohistochemical study of E-cadherin expression in human squamou cell carcinoma of the skin: relationship between decreased expression of Ecadherin in the primary lesion and regional lymph node metastasis. J Dermatol

Langer K, Konrad K, Wolff K: Keratitis, ichthyosis and deafness (KID) syndrome. report of three cases and a review of the literature. Br J Dermatol 122:689-697,

SW, Tomasetto C, Paul D, Keyomarsi K, Sager R: Transcriptional downregulation of gap-junction proteins blocks junctional communication in

SW, To

genes by subtractive hybridization. Proc Natl Acad Sci USA 88:2825-2829, 1991
gent
Madariaga J, Fromowitz F, Phillips M, Hoover HC Jr: Squamous cell carcinoma in congenital ichthyosis with deafness and keratitis. A case report and review of the literature. Cancer 57:2026-2029, 1986

McGrae JD Jr: Keratitis, ichthyosis, and deafness (KID) syndrome. Int J Dermato

29:89-93, 1990
Miller SA, Dykes DD, Polesky HF: A simple salting out procedure for extracting DNA from human nucleated cells. Nucleic Acids Res 16:1215, 1988

Moennikes O, Buchmann A, Willecke K, Traub O, Schwarz M. Hepatocarcinogenesis in female mice with mosaic expression of connexin32.

Morris MR, Namon A, Shaw GY, Panje WR, Mhoon EE: The keratitis, ichthyosis, and deafness syndrome. Otolaryngol Head Neck Surg 104:526-528, 1991

Nishida K, Adachi W, Shimizu-Matsumoto A, Kinoshita S, Mizuno K, Matsubara K, Okubo K: A gene expression profile of human corneal epithelium and the isolation of human keratin 12 cDNA. Invest Ophthalmol Vis Sci 37:1800-1809,
1996

Nurse DS: Keratitis, ichthyosis, deafness (KID) syndrome. Clin Exp Dermatol 19:280, 1994

Richard G, Smith LE, Bailey RA, et al: Mutations in the human connexin gene GJB3 cause erythrokeratodermia variabilis. Nat Genet 20:366-369, 1998

Rycroft RJ, Moynahan EJ, Wells RS: Atypical ichthyosiform erythroderma deafness and keratitis. A report of two cases. Br J Dermatol 94:211-217, 1976

and keratt1s. A report of two cases. Br J Dermatol 94:211-217, 1976
Saito T, Barbin A, Omori Y, Yamasaki H: Connexin 37 mutations in rat hepatic angiosarcomas induced by vinyl chloride. Cancer Res 57:375-377, 1997

Singal R, Tu ZJ, Vanwert JM, Ginder GD, Kiang DT: Modulation of the connexin26 tumor suppressor gene expression through methylation in human connexin26 tumor suppressor gene expression through methyl

Singh K: Keratitis, ichthyosis and deafness (KID syndrome). Australas J Dermatol 28:38-41, 1987

Skinner BA, Greist MC, Norins AL: The keratitis, ichthyosis, and deafness (KID) syndrome. Arch Dermatol 117:285-289, 1981

Stoler AB, Stenback F, Balmain A: The conversion of mouse skin squamous cell carcinomas to spindle cell carcinomas is a recessive event. J Cell Biol 122:11031117,1993

Tu ZJ, Kollander R, Kiang DT: Differential up-regulation of gap junction connexin 26 gene in mammary and uterine tissues. the role of Sp transcription factors. Mol Endocrinol 12:1931-1938, 1998

Van Steensel MA, Happle R, Steijlen PM: Molecular genetics of the hair follicle: the state of the art. Proc Soc Exp Biol Medical 223:1-7, 2000

Van Steensel MA, van Geel M, Steiljen PM: The molecular basis of hair growth. Eur J Dermatol 11:348-352, 2001

White TW: Functional analysis of human Cx26 mutations associated with deafness. Brain Res Brain Res Rev 32:181-183, 2000

White TW, Bruzzone R: Intercellular communication in the eye: clarifying the need for connexin diversity. Brain Res Brain Res Rev 32:130-137, 2000

Wilgoss A, Leigh IM, Barnes MR, et al: Identification of a novel mutation R42P in the gap junction protein beta-3 associated with autosomal do
erythrokeratoderma variabilis. J Invest Dermatol 113:1119-1122, 1999

Zhao HB, Santos-Sacchi J: Voltage gating of gap junctions in cochlear supporting cells: evidence for nonhomotypic channels. J Membr Biol 175:17-24, 2000 
Chapter 4 


\title{
Cutaneous Biology \\ HID and KID syndromes are associated with the same connexin 26 mutation
}

\author{
M.VAN GEEL, M.A.M.VAN STEENSEL, W.KÜSTER, ${ }^{*}$ H.C.HENNIES, $†$ R.HAPPLE, \\ P.M.STEIJLEN AND A.KÖNIG \\ Department of Dermatology, University Hospital Nijmegen, the Netherlands \\ *Department of Dermatology. TOMESA Fachklinik, Bad Salzschlirf. Germany \\ $\dagger$ Department of Molecular Genetics and Gene Mapping Centre, Max-Delbrueck-Centrum Berlin-Buch, Germany \\ $\ddagger$ Department of Dermatology, University Hospital Marburg, Germany \\ Accepted for publication 12 April 2002
}

Summary Background Keratitis-ichthyosis-deafness (KID) syndrome is a debilitating ectodermal dysplasia that predisposes patients to develop squamous cell carcinomas in addition to leading to profound sensory deafness and erythrokeratoderma. We recently demonstrated that KID can be caused by a specific missense mutation in connexin 26 (GJB2). Another syndrome, called hystrix-like ichthyosis-deafnesss (HID) syndrome, strongly resembles the KID syndrome. These disorders are distinguished mainly on the basis of electron microscopic findings. We hypothesized that KID and HID syndromes may be genetically related.

Objective To demonstrate by mutation analysis that HID and KID syndromes are genetically indistinguishable.

Methods DNA was extracted from paraffin-embedded tissue samples of the first HID syndrome patient described in the literature. Since the KID syndrome mutation abolishes an AspI restriction site, we were able to screen the patient's DNA by polymerase chain reaction and subsequent restriction enzyme analysis.

Results Restriction analysis of the connexin 26 gene in HID syndrome demonstrated the presence of the KID syndrome mutation that we previously described. This result was confirmed by direct DNA sequencing.

Conclusions We show that KID and HID syndromes are identical at the molecular level and confirm the clinical impression that these syndromes are one and the same. That previous clinical reports made a distinction may be a consequence of sampling artefacts; alternatively, genetic background effects such as the presence of concurrent mutations in other skin-expressed genes may modify the phenotype.

Key words: cancer, connexin, deafness, erythrokeratoderma, ichthyosis

Hystrix-like ichthyosis-deafness syndrome or HID syndrome (MIM 602540) is an autosomal-dominant inherited keratinizing disorder characterized by sensorineural deafness and spiky hyperkeratosis affecting the entire skin. The disease manifests itself shortly after

Correspondence: Michel van Geel, PhD, Department of Dermatology, University Medical Center Nijmegen, PO Box 9101, 6500 HB Nijmegen, the Netherlands

E-mail: m.vangeel@derma.azn.nl birth primarily with erythroderma. After the first year of life, the phenotype develops, with spiky and cobblestone-like hyperkeratosis covering the entire skin surface. Palms and soles are only mildly affected. Scarring alopecia can be part of the syndrome. A mild punctate keratitis has also been described in some patients. ${ }^{1-4} \mathrm{HID}$ is considered to differ from the similar autosomal-dominant keratitis-ichthyosis-deafness or KID syndrome (MIM 148210) in the extent and time of occurrence of skin symptoms and the severity of the associated keratitis. The KID syndrome, although the 
name suggests otherwise, is believed to represent a type of erythrokeratoderma with skin changes already present at birth. In addition, a severe palmoplantar keratoderma has been reported in patients suffering from KID syndrome. Electron microscopic features were also reported to differ between the two disorders. ${ }^{1,5-11}$ Otherwise the phenotypes are similar. ${ }^{12}$ An intriguing characteristic of both syndromes is the cancer predisposition. Both HID and KID syndrome patients show an increased incidence of squamous cell carcinoma. ${ }^{10,13-16}$ This unique symptom separates HID and KID syndromes from a group of disorders characterized by a very similar skin phenotype, the erythrokeratodermas. These skin diseases can also be associated with sensory deafness and can be caused by mutations in two members of the connexin gene family, specifically connexin 31 (GJB3 gene $)^{17}$ and connexin 30.3 (GJB4) ${ }^{18}$ Clouston syndrome (hidrotic ectodermal dysplasia 2, MIM 129500) is another disease associated with skin abnormalities, nail dystrophy and alopecia and is linked to mutations in connexin 30 (GJB6). ${ }^{19}$ Specific mutations in several connexins are also associated with non-syndromic sensorineural deafness. ${ }^{20}$ Connexins are membrane proteins, that assemble into hexameric hemichannels (connexons) that dock with a neighbouring hemichannel in an adjacent cell to form intracellular aqueous communication channels known as gap junctions. These are present in virtually all mammalian cells and serve as conduits for ions and small molecules (up to $1 \mathrm{kDa}$ ) allowing rapid exchange of information between cells. The protein structure is highly conserved among different mammalian species, especially at the membrane-bound and extracellular protein domains essential for correct folding, protein-protein interactions and voltage gating. ${ }^{21}$

Recently we identified a connexin 26 mutation in a patient diagnosed with KID syndrome. ${ }^{22}$ The missense mutation (D50N) is located at the first extracellular protein domain and may disrupt proper protein interactions between connexins or gap junction voltage gating. The same mutation was found in seven other KID patients. ${ }^{23}$ In addition, two other missense mutations in the first intracellular connexin 26 protein domain were found in two other patients. ${ }^{23}$

Here, we present evidence that KID and HID syndromes are both associated with an identical connexin 26 missense mutation and may represent a spectrum of phenotypic variability associated with one single gene mutation.

\section{Case report}

The patient represents the first case described in the literature. $^{1,24}$ Skin changes had been present since birth and were progressive. The patient suffered from severe bilateral sensory hearing loss. Keratitis was not present. The formation of multiple squamous cell carcinomas was noted since the age of 31 years. These tumours occurred predominantly on the lower legs. Treatment consisted of surgical removal and there was no evidence of recurrence after complete excision. The patient's son is also affected, suggesting autosomaldominant inheritance. ${ }^{4}$

Upon physical examination we saw spiky hyperkeratosis and sharkskin-like ichthyosis on the face and scalp. Dark-yellow to grey hyperkeratosis and erythro-

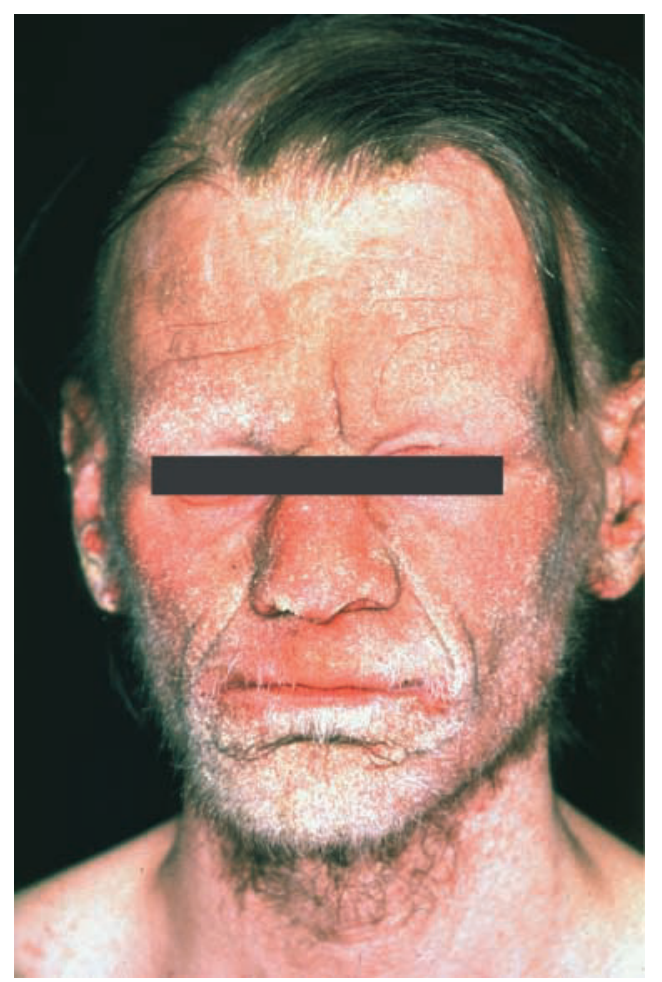

Figure 1. Extensive spiky hyperkeratosis covering most of the skin with a slightly erythrodermic aspect. Cobblestone-like hyperkeratosis is present on the scalp. Hypotrichosis of eyebrows, eyelids and scalp is likewise present. 


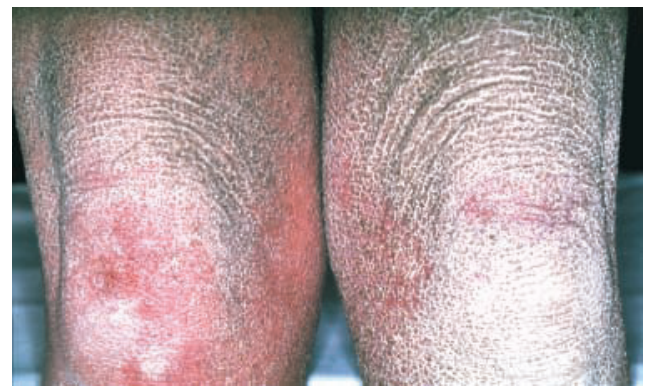

Figure 2. Erythroderma and impressive cobblestone-like hyperkeratosis around the knees that in some areas abruptly changes into a spiky hyperkeratosis.

derma were present over the entire skin surface (Fig. 1,2). The palms and soles were affected by a grey-brown hyperkeratosis. A hearing test performed by measuring EEG potentials revealed profound bilateral neurosensory hearing loss. Electron microscopic examination of skin biopsies demonstrated reduction of tonofilaments and the presence of membrane-bound granules containing an undefined mucous substance (data not shown), as previously described. ${ }^{1}$

\section{Methods}

\section{Mutation analysis}

Prompted by the clinical similarity between KID and HID syndromes, we examined genomic DNA extracted from paraffin-embedded tissue samples for the presence of a connexin 26 mutation. The DNA was extracted using the Puregene DNA isolation kit (Gentra systems, Minneapolis, MN U.S.A) according to the manufacturer's instructions. The single exon connexin 26 primer sequences were as follows: Cx26F, GCA TGC TTG CTT ACC CAG ACT C; Cx26R, AGG GGA GCA GAG CTC CAT TG; Cx26FS, CAG AAG GTC CGC ATC GAA GG; Cx26RS, GCT TCG AAG ATG ACC CGG AAG.

\section{Results}

Because the known KID mutation (148G $\rightarrow$ A; D50N) abolishes an AspI restriction site, we analysed this site by polymerase chain reaction and restriction digestion and demonstrated that the HID patient is heterozygous for lack of the restriction site (Fig. 3). The KID syndrome patient described previously ${ }^{22}$ shows an identical restriction pattern in contrast to her healthy

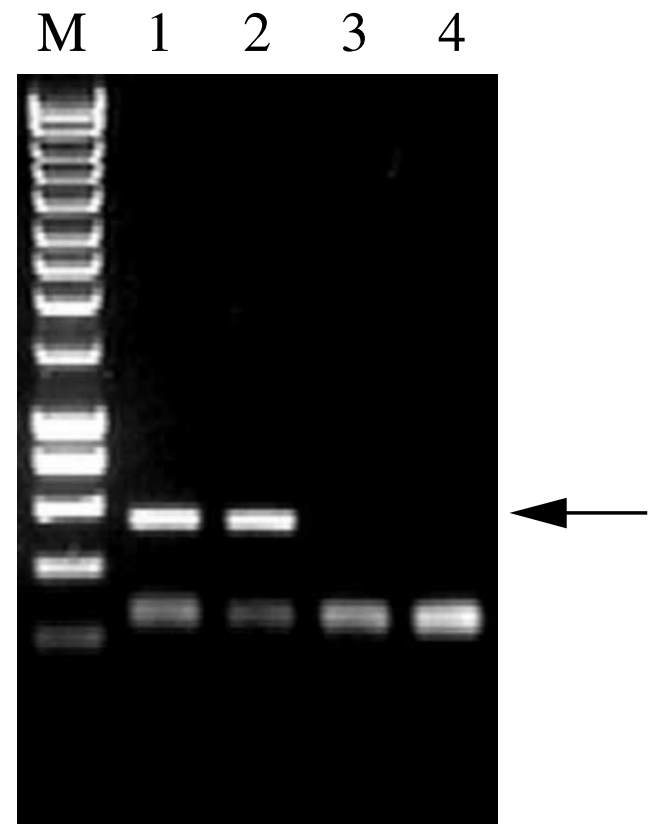

Figure 3. Asp I digests of connexin 26 polymerase chain reaction products (primers Cx26F-Cx26RS). Lane 1, Digest derived from HID syndrome patient; 2 , KID syndrome patient; ${ }^{22} 3$, healthy brother of KID patient; ${ }^{22} 4$, KID patient's unaffected mother. ${ }^{22} \mathrm{M}$ indicates DNA size markers; arrow indicates undigested mutant allele $(550 \mathrm{bp}$, digested products: 295 and 255 bp).

family members (Fig. 3). Subsequently, we confirmed the presence of the mutation by direct sequencing and found no additional variations in the connexin 26 gene (data not shown). The sequences were assembled and analysed with the Phred-Phrap-Consed software tools. ${ }^{25-27}$ The mutation was a $\mathrm{G} \rightarrow \mathrm{A}$ transition designated 148G $\rightarrow \mathrm{A}$, causing an aspartic acid to asparagine change at codon 50 (D50N). This mutation has been found previously by this group and others $^{22,23}$ in patients diagnosed with KID syndrome.

\section{Discussion}

We present evidence that KID and HID syndromes are identical on the molecular level: patients with both disorders carry the heterozygous missense mutation D50N in the highly conserved first extracellular domain of connexin 26. The disputed ${ }^{12}$ clinical distinction between the two syndromes was based on age at onset of symptoms and a supposed lack of involvement of 
palms and soles. ${ }^{12}$ The latter symptom is not a reliable indicator, as palm and sole involvement in KID syndrome is quite variable. ${ }^{6}$ The keratitis in HID syndrome is supposed to be less severe than that found in KID syndrome, but the number of reports available for examination preclude firm conclusions in this area. Moreover, keratitis is in itself a rather non-specific symptom that does not serve well to differentiate disease entities. Ultrastructural features were previously reported to distinguish the two disorders. Specific changes in HID syndrome were reported to consist of reduction of tonofilaments in the epidermis and excess formation of so-called 'mucous granules' ${ }^{1}$ and accumulation of this undefined mucous material in the intercellular spaces of the epidermis. However, there is a report of similar abnormalities in patients diagnosed with KID syndrome. ${ }^{28}$ Failure to find them in other KID patients may be a sampling artefact as the erythrokeratoderma has a regional distribution, with some skin areas being more severely affected than others. It is conceivable that the presence of electron microscopic abnormalities correlates with the presence of severe skin symptoms. Despite the tenuous clinical evidence for a distinction between HID and KID syndromes, variability in clinical presentation of KID syndrome exists. Genetic background effects such as concurrent mutations and polymorphisms in other skin-expressed genes can explain this phenomenon. Such digenic mutation effects have been previously observed for connexin-associated forms of deafness. ${ }^{29}$ Nevertheless, there are no solid clinico-pathological arguments to differentiate the disorders. This notion is now confirmed by our finding of the KID syndrome-associated D50N mutation in the connexin 26 gene in our patient.

In conclusion, we show that HID syndrome is genetically identical to KID syndrome. This result confirms the clinical impression that KID and HID syndromes represent a single disease entity.

\section{Acknowledgments}

M.van Geel is supported by a grant from Multigen BV, the Netherlands. M.A.M.van Steensel is supported by grants from ZON-MW (920-03-085), Multigen BV, the Netherlands and the Berliner Stiftung für Dermatologie.

\section{References}

1 Gulzow J, Anton-Lamprecht I. [Ichthyosis hystrix gravior typus Rheydt: ein otologisch-dermatologisches syndrom]. Laryngol Rhinol Otol (Stuttg) 1977; 56: 949-55.
2 Baden HP, Bronstein BR. Ichthyosiform dermatosis and deafness: report of a case and review of the literature. Arch Dermatol 1988; 124: $102-6$.

3 Badillet C, Blanchet-Bardon C, Cabral O et al. Etude mycologique de trois cas d'erythrodermie avec keratite et surdite (ichtyose de Rheydt). Bull Soc Mycol Med (Paris) 1982; 11: 191-8.

4 König A, Küster W, Berger R et al. Autosomal dominant inheritance of HID syndrome (hystrix-like ichthyosis with deafness). Eur J Dermatol 1997; 7: 554-5.

5 Tercedor J, Garcia A. [Keratotic neurocutaneous syndromes]. Rev Neurol 1997; 25 (Suppl. 3): S238-42.

6 Caceres-Rios H, Tamayo-Sanchez L, Duran-Mckinster C et al. Keratitis, ichthyosis, and deafness (KID syndrome): review of the literature and proposal of a new terminology. Pediatr Dermatol 1996; 13: 105-13.

7 Wilson GN, Squires RH Jr, Weinberg AG. Keratitis, hepatitis, ichthyosis, and deafness: report and review of KID syndrome. Am J Med Genet 1991; 40: 255-9.

8 Langer K, Konrad K, Wolff K. Keratitis, ichthyosis and deafness (KID)-syndrome, report of three cases and a review of the literature. Br J Dermatol 1990; 122: 689-97.

9 Puig L, Moreno A, Perez M et al. [KID syndrome (keratitis-ichthyosis-deafness)]. Med Cutan Ibero Lat Am 1987; 15: 223-8.

10 Madariaga J. Fromowitz F. Phillips M et al. Squamous cell carcinoma in congenital ichthyosis with deafness and keratitis. A case report and review of the literature. Cancer 1986; 57: 2026-9.

11 Harms M, Gilardi S, Levy PM et al. KID syndrome (keratitis, ichthyosis, and deafness) and chronic mucocutaneous candidiasis: case report and review of the literature. Pediatr Dermatol 1984; 2: $1-7$.

12 Nousari HC, Kimyai-Asadi A, Pinto JL. KID syndrome associated with features of ichthyosis hystrix. Pediatr Dermatol 2000; 17: $115-17$.

13 Hazen PG, Walker AE, Stewart JJ et al. Keratitis, ichthyosis, and deafness (KID) syndrome: management with chronic oral ketoconazole therapy. Int J Dermatol 1992; 31: 58-9.

14 Morris MR, Namon A, Shaw GY et al. The keratitis, ichthyosis, and deafness syndrome. Otolaryngol Head Neck Surg 1991; 104: $526-8$.

15 Hazen PG, Carney P, Lynch WS. Keratitis, ichthyosis, and deafness syndrome with development of multiple cutaneous neoplasms. Int J Dermatol 1989; 28: 190-1.

16 Grob JJ. Breton A. Bonafe JL et al. Keratitis, ichthyosis, and deafness (KID) syndrome. Vertical transmission and death from multiple squamous cell carcinomas. Arch Dermatol 1987; 123: 777-82.

17 Richard G, Smith LE, Bailey RA et al. Mutations in the human connexin gene GJB3 cause erythrokeratodermia variabilis. Nat Genet 1998; 20: 366-9.

18 Macari F, Landau M, Cousin P et al. Mutation in the gene for connexin 30.3 in a family with erythrokeratodermia variabilis. Am I Hum Genet 2000; 67: 1296-301.

19 Lamartine J, Munhoz Essenfelder G, Kibar Z et al. Mutations in GJB6 cause hidrotic ectodermal dysplasia. Nat Genet 2000; 26: $142-4$.

20 Petit C, Levilliers J, Hardelin JP. Molecular genetics of hearing loss. Annu Rev Genet 2001; 35: 589-646.

21 Richard G. Connexins: a connection with the skin. Exp Dermatol 2000; 9: 77-96.

22 van Steensel MA, van Geel M, Nahuys M et al. A novel connexin 26 mutation in a patient diagnosed with keratitis-ichthyosisdeafness syndrome. J Invest Dermatol 2002; 118: 724-7. 
M.VAN GEEL et al.

23 Richard G, Rouan F. Willoughby CE et al. Missense mutations in GJB2 encoding connexin-26 cause the ectodermal dysplasia keratitis-ichthyosis-deafness syndrome. Am J Hum Genet 2002; 70: $1341-8$.

24 Schnyder UW, Gloor M. Ichthyosis hystrix gravior Typus Rheydt (Ichthyosis hystrix gravior mit praktischer Taubheit). Z Hautkr 1977; 52: 763-6.

25 Ewing B, Green P. Base-calling of automated sequencer traces using phred. II. Error probabilities. Genome Res 1998; 8: 186-94.

26 Ewing B, Hillier L, Wendl MC et al. Base-calling of automated sequencer traces using phred. I. Accuracy assessment. Genome Res $1998 ; 8$ : $175-85$.
27 Gordon D, Abajian C, Green P. Consed: a graphical tool for sequence finishing. Genome Res 1998; 8: 195-202.

28 Jurecka W, Aberer E, Mainitz M et al. Keratitis, ichthyosis, and deafness syndrome with glycogen storage. Arch Dermatol 1985; 121: 799-801.

29 Kelsell DP, Wilgoss AL, Richard G et al. Connexin mutations associated with palmoplantar keratoderma and profound deafness in a single family. Eur J Hum Genet 2000; 8: 141-4. 
Chapter 5 
CORRESPONDENCE

connexin genes $(G J B)$ have shown that these genes have a tissue-specific distribution. Several groups have shown that mutations in connexin 31 (GJB3) and connexin 30.3 (GJB4) are associated with the autosomal dominant skin disorder erythrokeratoderma variabilis of Mendes da Costa (EKV). ${ }^{1-3}$ The phenotype of this debilitating skin disorder is characterized by early onset of sharply demarcated scaly erythematous skin lesions that tend to migrate across the body. Sensorineural deafness can be part of the phenotype and is caused by specific mutations. ${ }^{3}$ Progressive symmetric erythrokeratodermia (PSEK) shares many features with EKV and both phenotypes are considered to be manifestations of the same basic disorder. ${ }^{4}$

We present a 4-year-old boy who first presented to our outpatient dermatology clinic at the age of 2 years with patchy redness and scaling of the skin. These abnormalities had been present since birth but worsened after 8 months of age. According to the parents, the patches initially appeared on the face and later spread across the entire body. The family history was negative for similar disorders. The non-consanguineous parents were of Dutch descent. Physical examination at 2 years of age showed symmetrical, sharply demarcated gyrated red patches on both cheeks with slight whitish scaling and induration. Light and electron microscopic examination of the lesions showed acanthosis, hyperkeratosis, parakeratosis, and a slight inflammatory infiltrate in the dermis. No clumping of tonofilaments or acantholysis was seen (data not shown). During follow-up, the skin lesions spread across the body, starting with the extremities (Fig. 1). The keratoderma did not respond to topical treatment and subsequently oral acitretin (Neotigason ${ }^{\circledR}$; Roche) $0.5 \mathrm{mg} \mathrm{kg}^{-1}$ daily was given. Response to this treatment was satisfactory. There were no clinical suspicions of hearing loss. The results of the physical examination, history and light and electron microscopy are consistent with the EKV/PSEK phenotype.

Genomic DNA from peripheral blood leucocytes was isolated from the parents, the patient and his sister. The connexin 30.3, 31, 31.1, 30, 26 and loricrin genes were analysed for mutations by polymerase chain reaction (PCR) and direct sequencing. In the patient a heterozygous $94 \mathrm{C} \rightarrow \mathrm{T}$ change in codon 32 of connexin 31 was identified, changing a conserved arginine to a tryptophan (R32W). In addition, a homozygous 4-bp deletion (154-157delGTCT) in GJB4 was detected (Fig. 2A). The nucleotide changes in GJB3 and GJB4 eliminate an $\mathrm{NciI}$ and an AspI restriction site, respectively. Subsequent restriction analysis showed both parents and the maternal grandfather to be heterozygous for the GJB4 deletion (Fig. 2B). The mother and maternal grandfather were both heterozygous for the GJB3 variation. The patient's GJB3/GJB4 genotype was identical to that of his unaffected sister, excluding either DNA variation as causative for the disease. However, we cannot exclude modifier genes or external factors triggering the disease phenotype in the context of this connexin genotype. Subsequently, we examined 84 unrelated controls and found five heterozygotes for the GJB4 deletion (allele frequency 0.03) and three for the

SIR, Connexins are the major protein components of gap junctions, intercellular communication channels that are vital for cell growth and differentiation in various tissues. During the past few years, mutations in several differen 


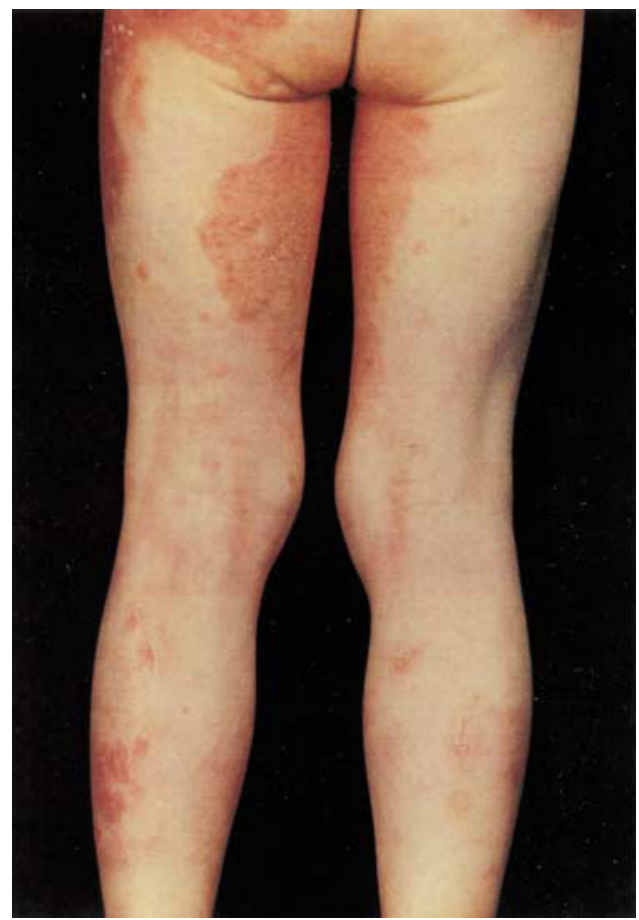

Figure 1. Clinical skin features of the patient with erythrokeratoderma variabilis/progressive symmetric erythrokeratodermia (see main text for description).

GJB3 variation $(0 \cdot 02)$. Both variations apparently represent normal polymorphisms in the Dutch population. All genotypes were confirmed by sequence analysis.

The deletion in GJB4 leads to a frameshift and consequently a nonsense codon resulting in a non-functional protein product that is truncated after the first transmembrane domain. The GJB4 deletion does not induce nonsensemediated RNA decay, as we could detect mRNA transcripts in a skin biopsy from the patient by reverse transcription-PCR analysis (data not shown). It has been shown that connexin 31 knockout mice do not have a phenotype except for a transient placental dysmorphism. ${ }^{5}$ Apparently, other connexins can compensate for the lost proteins. Our patient and his sister show that this is true for humans also, as they effectively represent connexin 30.3 knockouts. It also shows that connexin 30.3 is not required for normal skin function or embryonic development in humans.

The nucleotide transition in connexin 31 changes a highly conserved arginine to a tryptophan in the first transmembrane domain $(\mathrm{R} 32 \mathrm{~W})$. Kelsell et al. have previously described this nucleotide change in a family with palmoplantar keratoderma and hearing defects. ${ }^{6}$ They suggested that it

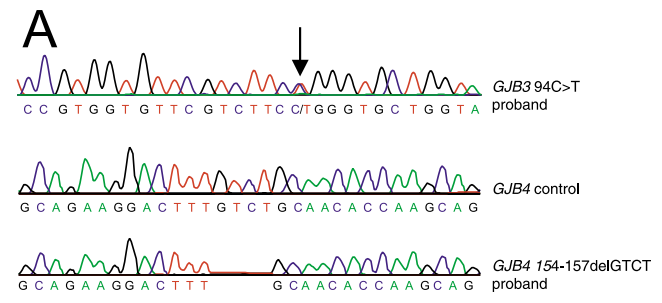

B

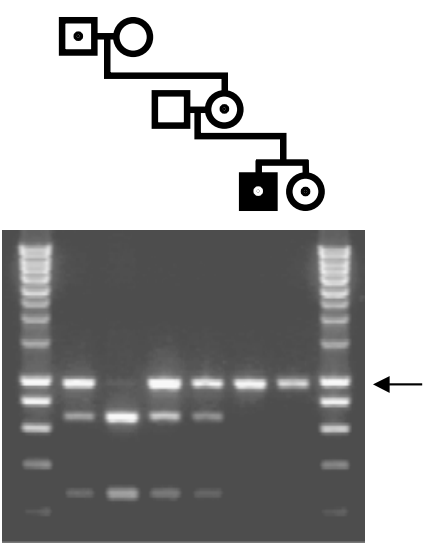

Figure 2. Sequence analysis of GJB3 and GJB4 in a family with a sporadic case of erythrokeratoderma variabilis $(\mathrm{EKV}) /$ progressive symmetric erythrokeratodermia (PSEK). (A) Sequence traces of the patient's GJB3 heterozygous $94 \mathrm{C} \rightarrow \mathrm{T}$ variation and GJB4 homozygous 154-157delGTCT deletion compared with wild-type. (B) Polymerase chain reaction-AspI restriction analysis of DNA samples from the EKV/PSEK family. Both the patient and his healthy sister are homozygous for the GJB4 deletion (arrow, 948 bp; digested fragments, 674 and 278 bp). Lanes 1 and 8 , markers; lane 2, grandfather; lane 3 , grandmother; lane 4, father; lane 5, mother; lane 6, affected son; lane 7, unaffected daughter. Family members indicated with a dot are heterozygous for the GJB3 variation (restriction analysis not shown).

contributed to the high-frequency hearing loss observed in some family members but also mentioned that it might be a polymorphism. Our findings now confirm those by LópezBigas et al., who found the variation in $17 \%$ of normal individuals examined. ${ }^{7}$ However, the major difference in allele frequency suggests that the variation is less common in the Dutch population than in the Spanish population.

Our results demonstrate that EKV/PSEK is a genetically heterogeneous group of disorders. Connexin mutations are detected in some typical cases of EKV, ${ }^{1,2}$ while other cases lack mutations. ${ }^{3}$ No mutations are described in typical cases of PSEK. The PSEK patient described by Ishida-Yamamoto et al. is not typical and in our opinion probably represents a case of Vohwinkel syndrome. ${ }^{8}$ Analysis of connexin 30.3, 31 , $31.1,30,26$ and loricrin did not identify causative mutations 
in our patient. These findings confirm the previous report about the genetic heterogeneity of the EKV/PSEK phenotype. $^{3}$

Department of Dermatology, University Medical

Center Nijmegen,

Rene Descartesdreef 1 ,

6525 GL Nijmegen,

the Netherlands

E-mail:m.vangeel@derma.azn.nl

\section{References}

1 Richard G, Smith LE, Bailey RA et al. Mutations in the human connexin gene GJB3 cause erythrokeratodermia variabilis. Nat Genet 1998; 20: 366-9.

2 Macari F, Landau M, Cousin P et al. Mutation in the gene for connexin 30.3 in a family with erythrokeratodermia variabilis. $\mathrm{Am}$ J Hum Genet 2000; 67: 1296-301.

3 Kelsell DP. Di WL, Houseman MJ. Connexin mutations in skin disease and hearing loss. Am J Hum Genet 2001; 68: 559-68.

4 Macfarlane AW, Chapman SJ, Verbov JL. Is erythrokeratoderma one disorder? A clinical and ultrastructural study of two siblings. Br J Dermatol 1991; 124: 487-91.

5 Plum A, Winterhager E, Pesch J et al. Connexin31-deficiency in mice causes transient placental dysmorphogenesis but does not impair hearing and skin differentiation. Dev Biol 2001; 231: 334 47 .

6 Kelsell DP, Wilgoss AL, Richard G et al. Connexin mutations associated with palmoplantar keratoderma and profound deafness in a single family. Eur J Hum Genet 2000; 8: 469-72.

7 López-Bigas N, Rabionet R, Arbones ML, Estivill X. R32W variant in connexin 31: mutation or polymorphism for deafness and skin disease? Eur J Hum Genet 2001: 9: 70.

8 Ishida-Yamamoto A, McGrath JA, Lam H et al. The molecular pathology of progressive symmetric erythrokeratoderma: a frameshift mutation in the loricrin gene and perturbations in the cornified cell envelope. Am J Hum Genet 1997; 61: 581-9. 
Chapter 6 


\title{
Clouston Syndrome Can Mimic Pachyonychia Congenita
}

\author{
Maurice A.M. van Steensel, Marcel F. Jonkman, ${ }^{*}$ Michel van Geel, Peter M. Steijlen, W.H. Irwin McLean, $\dagger$ \\ and Frances J.D. Smith $\dagger$ \\ Department of Dermatology, University Medical Center Nijmegen, Nijmegen, The Netherlands; *Department of Dermatology, University of Groningen, \\ Groningen, The Netherlands; †Epithelial Genetics Group, Human Genetics Unit, Ninewells Medical School, University of Dundee, Dundee, UK
}

We studied three families suffering from nail abnormalities who had previously been diagnosed as pachyonychia congenita. No keratin gene mutations were detected. Sequencing of connexin 30 (GJB6 gene) in these patients identified heterozygous missense mutations G11R and A88V that are known to be associated with Clouston syndrome. This unexpected finding expands the Clouston syndrome phenotype and suggests that some patients diagnosed with pachyonychia may in fact be suffering from Clouston syndrome. Key words: connexin 30/genodermatosis/GJB6/nail dystrophy/pachyonychia congenita. J Invest Dermatol 121:1035-1038, 2003

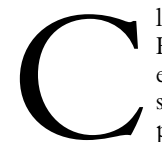

louston syndrome (hidrotic ectodermal dysplasia, HED, OMIM 129500) is an autosomal dominan ectodermal dysplasia characterized by hypotrichosis severe nail dystrophy, and often palmoplantar hyperkeratosis as well as hyperpigmentation of the skin over large joints (Rajagopalan and Tay, 1977; Fraser and Der Kaloustian, 2001). Teeth and eccrine gland function are normal. Sensorineural deafness can be part of the phenotype; mental retardation has also been described (Copeland et al, 1977). The degree of the alopecia and the hyperkeratosis are apparently variable, with lack of the latter having been reported in one family. Mutations in the gap junction protein connexin 30 (GJB6 gene) have been found in several families of different ethnic origins. Almost all families described so far have inherited one of two recurrent missense mutations, G11R or A88V (Lamartine et al, 2000). Recently, we have described a novel connexin $30 \mathrm{mu}-$ tation, V37E, in a sporadic case of Clouston syndrome (Smith et al, 2002).

Pachyonychia congenita (PC) is a group of ectodermal dysplasias whose most obvious phenotypic characteristic is hypertrophic nail dystrophy. Some forms of PC are known to be caused by mutations in differentiation-specific keratins. There are two main types of the disease: type 1 (PC-1) where nail dystrophy is often accompanied by focal keratoderma and sometimes oral leukokeratosis (Jadassohn and Lewandowsky, 1906); and type 2 (PC-2) where there are a variable number of additional features including pilosebaceous cysts, natal teeth, and angular chelosis (Jackson and Lawler, 1951). The PC-1 phenotype is associated with mutations in keratins 6a and 16 (Bowden et al, 1995; McLean et al, 1995), whereas the PC-2 phenotype has been linked to mutations in keratins 6b and 17 (McLean et al, 1995; Smith et al, 1998).

Here we describe three patients, all originally diagnosed with variant forms of PC. The oldest patient was suffering from what was originally thought to be a new type of PC consisting of mild thickening of the nails associated with hypotrichosis universalis.

Manuscript received October 24, 2002; revised April 8, 2003; accepted for publication May 5, 2003

Reprint requests to: Dr Frances J. D. Smith, Human Genetics Unit, Ninewells Medical School, University of Dundee, Dundee DD1 9SY, UK. Email: fjsmith@hgmp.mrc.ac.uk

Abbreviation: PC, pachyonychia congenita.
The other two patients were suffering from pachyonychia only. Hearing was normal in all three patients. An unexpected finding of a connexin 30 mutation in these patients indicates that the diagnosis should be Clouston syndrome, warranting evaluation of previously described "new" hair-nail dysplasias. All patients were seen with informed consent and all investigations with the approval of the appropriate local Medical Ethics Committees.

\section{MATERIALS AND METHODS}

Mutation analysis Genomic DNA was extracted from whole blood by standard procedures. A $1104 \mathrm{bp}$ fragment spanning the full length of the GJB6 gene (connexin 30) was amplified with primers Cx30P1 (forward) $5^{\prime}$ GGC AGG GAG TTG AAG TTG TAA $3^{\prime}$ and Cx30P2 (reverse) $5^{\prime}$ ACG TTG TGT ATG AAT GGA GCA $3^{\prime}$ as previously described (Smith et al, 2002). PCR products were purified using the Qiaquick PCR purification kit (Qiagen, Crawley, UK) and sequenced on an ABI 3100 automated DNA sequencer (ABI, Foster City, CA) using primers $\mathrm{Cx} 30 \mathrm{P} 1$, Cx30P2 (above), and Cx30P7 (reverse) 5' GAC CCC TCT ATC CGA ACC TT $3^{\prime}$.

Mutation G11R does not create or destroy any restriction site so a primer was designed with a mismatch to create a new Bcll site in combination was designed with a mismatch to create a new Bcll site in combination
with the mutation. Genomic DNA was amplified using primers Cx30Bcl with the mutation. Genomic DNA was amplified using primers Cx30Bcll
(forward) $5^{\prime}$ TGG ATT GGG GGA CGC TGC ACA CTT TGA TC $3^{\prime}$ (forward) $5^{\prime}$ TGG ATT GGG GGA CGC TGC ACA CTT TGA TC $3^{\prime}$
(mismatched base underlined) and Cx30P8 (reverse) $5^{\prime}$ CAC TTC CTG (mismatched base underlined) and Cx30P8 (reverse) $5^{\prime}$ CAC TTC CTG
GGC AGC CAC CAC GAG GAT CAT $3^{\prime}$ in standard PCR buffer containing $1.5 \mathrm{mM} \mathrm{MgCl}$ and $4 \%$ dimethylsulfoxide. PCR conditions were $94^{\circ} \mathrm{C} 5 \mathrm{~min} \times 1 ; 94^{\circ} \mathrm{C} 30 \mathrm{~s}, 55^{\circ} \mathrm{C} 45 \mathrm{~s}, 72^{\circ} \mathrm{C} 1 \mathrm{~min} \times 35$; and $72^{\circ} \mathrm{C}$ $5 \mathrm{~min} \times 1$. The resultant $128 \mathrm{bp}$ fragment was digested with $10 \mathrm{U}$ Bcl (New England Biolabs) at $50^{\circ} \mathrm{C}$ for at least $4 \mathrm{~h}$. Digests were analyzed on $3 \%$ agarose minigels.

Mutation A88V abolishes an HaeII site. Genomic DNA was amplified with $\mathrm{C} \times 30 \mathrm{P} 1$ and $\mathrm{C} \times 30 \mathrm{P} 2$ (as above) and PCR products were digested with $8 \mathrm{U}$ HaeII (New England Biolabs) at $37^{\circ} \mathrm{C}$ for a minimum of $4 \mathrm{~h}$ Digests were analyzed on $1.5 \%$ agarose minigels.

\section{RESULTS}

Clinical findings Two of the families in this study have been reported previously. Briefly, the proband in family 1 (van Steensel et al, 2001) is a 63-y-old Dutch male, who was originally diagnosed with a new variant of PC, consisting of thickening of the nails (Fig 1a) and almost universal 

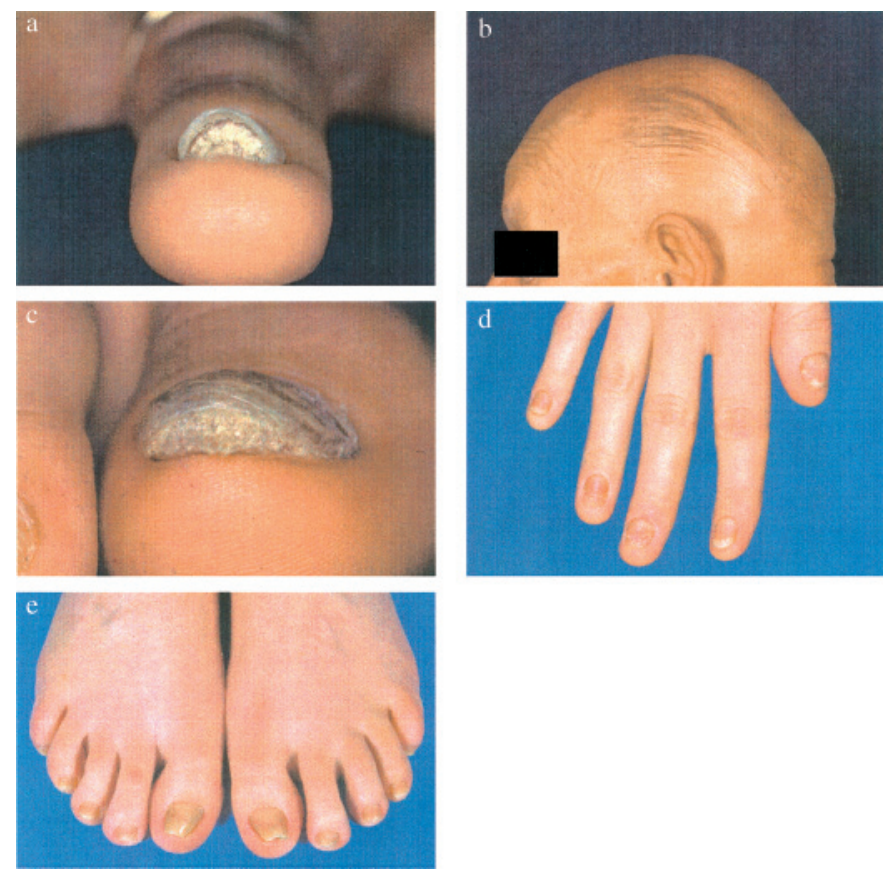

Figure 1. Clinical findings. (a) Fingernail of the proband in family 1 , showing subungual hyperkeratosis and severe curvature of the nail. These features strongly resemble the type of nail dystrophy observed in PC due to keratin mutations; however, in this patient the causative mutation is G11R in the connexin 30 gene. (b) The proband of family 1 (shown here) and other affected individuals in this kindred had nearly complete alopecia. Only a few remaining hairs in the temporal region can be seen. (c) Severe hypertrophic nail dystrophy of the toenails in the proband from family 2 . This patient carries the heterozygous missense mutation G11R in the connexin 30 gene. (d) Fingernails of the proband in family 3 showing some thickening of the nail plate, distal onycholysis, subungual hyperkeratosis, and paronychia. (e) Toenails of the proband in family 3 showing thickening and abnormal curvature of the nails. This patient carries heterozygous missense mutation $\mathrm{A} 88 \mathrm{~V}$ in the connexin 30 gene. hypotrichosis (Fig $\mathbf{1}$ b). The alopecia and nail phenotype was entirely consistent throughout affected members of the family. No tooth abnormalities were noted and sweating was normal. Notably, hearing was normal and deafness did not occur elsewhere in the family. Several family members were affected in a manner consistent with autosomal dominant inheritance. DNA was available for study only from the proband.

The proband in family 2, a 13-y-old male of Moroccan descent, was diagnosed with PC without other abnormalities (Fig 1c). His parents were not consanguineous. The case wa previously described in Chang et al (1994). Several members of his family were also affected with a combination of nail thickening, yellow discoloration of nails, and subungual hyperkeratosis. The abnormalities appeared during the first few month of life. The nail changes were identical to those seen in PC associated with keratin mutations; hence the disorder was considered as a possible allelic variant. Due to problems of patient consent and access, DNA was only available from the proband.

The proband in family 3 was a 25 -y-old Dutch woman, who was also first diagnosed with a variant of PC due to nail deformities (pincer nails, subungual hyperkeratosis, and chronic paronychia) (Fig 1d,e). Her fingernails were all divergent with thickening of the nail plate, distal onycholysis, subungual hyperkeratosis, and paronychia. The toenails were thickened and curved and she suffered from very mild focal keratoderma of the soles, mainly on the heels (not shown). There was no alopecia, the eyebrows were sparse laterally, and the evelashes were both thin and sparse. Hearing was normal. Since 1996, the proband has suffered from relapsing infections of the fingernails, which began as green discoloration with pus formation underneath the nail folds. She has been treated with ciprofloxacine and itroconazol and uses latex gloves for contact with water. As a child she was admitted to hospital because of patchy alopecia of the scalp which had been interpreted as alopecia areata. Her mother had thickened toenails and excessive callus formation on the soles, suggesting dominant inheritance. No other family members were affected. DNA was available only from the proband.

Identification of connexin $\mathbf{3 0}$ mutations $\mathrm{PC}$ has previously been shown to be caused by dominant-acting mutations in differentiation-specific keratin genes, K6a, K6b, K16, and K17, that are expressed in the nail bed and other ectodermal structures (Bowden et al, 1995; McLean et al, 1995; Smith et al, 1998). We screened these keratin genes as previously described (Smith et al, 1999a; 1999b; Terrinoni et al, 2001); however, we found no mutations in any of the patients (data not shown). The GJB6 gene (connexin 30) was chosen for screening because of the pachyonychia-like nail changes we observed in our recently reported Clouston syndrome patient, who had a novel connexin 30 mutation (Smith et al, 2002).

In both families 1 and 2 we found a heterozygous missense transition mutation $31 \mathrm{G} \rightarrow \mathrm{A}$ by direct sequencing of PCR products. The mutations predict the substitution of an arginine for a glycine at codon 11 of the connexin 30 polypeptide (G11R), as shown in Fig 2. This mutation, in combination with a mismatch primer, creates a novel $B c l$ r restriction enzyme site, which was used to confirm the mutation in connexin $30 \mathrm{PCR}$ fragments by restriction digestion. This mutation has been previously reported in nine out of 12 classical Clouston syndrome kindreds of various ethnic backgrounds (Lamartine et al, 2000).

In family 3 , we identified a heterozygous missense mutation $263 \mathrm{C} \rightarrow \mathrm{T}$, which leads to the predicted amino acid change $\mathrm{A} 88 \mathrm{~V}$ in the connexin 30 protein (Fig 2). This mutation has also been reported previously in three cases of Clouston syndrome (Lamartine et al, 2000). Mutation A88V deletes an HaeII restriction enzyme site, which was used to confirm the mutation by restriction digestion (data not shown). Both mutations were excluded from 50 control genomic DNA samples by the restriction digests described above (data not shown). 


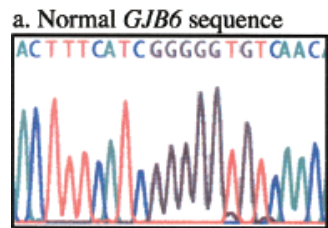

b. GJB6 mutation G11R

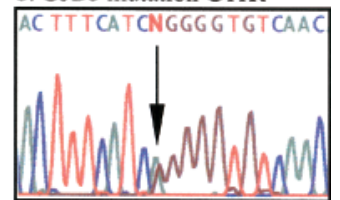

c. Normal GJB6 sequence

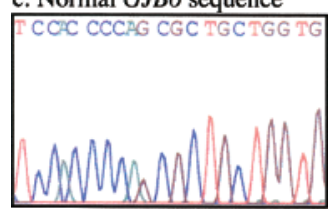

d. GJB6 mutation $\mathrm{A} 88 \mathrm{~V}$

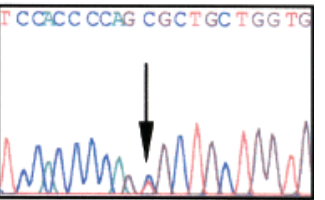

Figure 2. Molecular genetic analysis. (a) Normal GBJ6 sequence corresponding to codons 8-14. (b) The same region of the GBJ6 gene as shown in (a) from the proband in family 1 showing heterozygous missense mutation $31 \mathrm{G} \rightarrow \mathrm{A}$ (arrow) predicting the amino acid change G11R (sequencing data from family 2 not shown). (c) Normal GBJ6 sequence corresponding to codons 85-91. (d) The same region of the GBJ6 gene as shown in (c) from the proband in family 3 showing heterozygous missense mutation $263 \mathrm{C} \rightarrow \mathrm{T}$ (arrow) that predicts amino acid change A88V.

\section{DISCUSSION}

Here, we describe three families where the original diagnosis was that of variant types of PC. Upon mutation analysis, these patients all have genetic defects in connexin 30 that have been shown previously to cause hidrotic ectodermal dysplasia, also known as Clouston syndrome (Lamartine et al, 2000). Apparently, Clouston syndrome can have many guises. The phenotype is usually described as consisting of hypotrichosis; thick, ridged, and extremely short nails; palmoplantar hyperkeratosis; and hyperpigmentation of skin over large joints. There is one report of a family with Clouston syndrome lacking the palmoplantar hyperkeratosis but this symptom is not generally regarded as part of the phenotype (Hassed et al, 1996). Palmoplantar hyperkeratosis with hypotrichosis is also seen in a few cases of PC (Templeton and Wiegand, 1997) and other ectodermal dysplasias without pachyonychia (van Steensel et al, 2002). In all the pachyonychia patients whom we have studied where mutations in keratin genes have been identified, however, there have been no instances of hypotrichosis or alopecia. Thus, the presence of alopecia in addition to hypertrophic nail dystrophy may be indicative of a connexin defect, rather than a keratin mutation. Thickening of the nails, shown here to be clinically very similar to the nail changes seen in PC, can apparently also be a manifestation of Clouston syndrome. Phenotypic variation has been reported previously in relation to connexin mutations in both skin disease and hearing loss, as reviewed recently (Kelsell et al, 2001; Richard, 2001a; 2001b). Specifically, in GJB2 missense mutations D66H and R75W have been shown to have variable effects on the skin and frameshift mutation $35 \mathrm{delG}$ gives rise to variable degrees of hearing loss. Thus, the phenotypic variability reported here, i.e., nail dystrophy with or without alopecia, is likely to be part of a general phenomenon in the human connexin disorders.

In two patients, both of whom had been previously reported as having variant types of PC (Chang et al, 1994; van Steensel et al, 2001), we demonstrated the presence of mutation G11R in connexin 30. Family 1 also had associated universal hypotrichosis. Complete hypotrichosis has been described in Clouston syndrome (Smith et al, 2002) and in hindsight the diagnosis should have been entertained in this patient. In families 2 and 3, however, affected family members had isolated pachyonychia in the absence of other ectodermal abnormalities. These families were more readily confused with pachyonychia, particularly the PC-1 (Jadassohn-Lewandowsky) variant, due to K6a/K16 mutations, where the other ectodermal features, leukoplakia and focal keratoderma, may be mild or absent in some cases. Connexin $30 \mathrm{mu}-$ tations G11R and A88V were identified in families 2 and 3, respectively.

It is of interest to note that the same mutation can have disparate effects on hair, nails, and palmoplantar skin. At present, we have no explanation for this observation; however, the genetic background of different families and individual patients is likely to play a role. It is possible that polymorphisms in keratins, connexins, or other genes encoding epithelial structural molecules could influence the phenotype. From the clinical phenotype alone, it is not possible to distinguish the Clouston syndrome nail dystrophy from the ones associated with keratin mutations. No nail biopsies have been performed in our patients; it is therefore not possible to establish a histologic correlation between genotype and phenotype.

Given our findings, it can be expected that some disorders characterized by thick or brittle nails with or without hair abnormalities may turn out to be phenotypic variants of Clouston syndrome. There exist several reports of patients suffering from hair and/or nail dysplasias that have been classified as new disorders (Calzavara-Pinton et al, 1991; Pinheiro and Freire-Maia, 1992; Christianson and Fourie, 1996). We suggest that some of these patients could in fact be suffering from Clouston syndrome and that they should be examined for connexin 30 mutations. As connexin 30 is encoded by a small single exon gene, mutation screening is both straightforward and inexpensive and should perhaps be considered as part of the mutation screening protocols for pachyonychia. Similarly, Clouston syndrome should be considered as part of the differential diagnosis for pachyonychia and vice versa. It should be noted that we have in fact sequenced a few patients diagnosed as having a form of PC for all four keratins involved in PC, i.e., K6a, K6b, K16, and K17, and also connexins 26 and 30 . These patients did not have mutations in any of these genes. Therefore, there are other as yet unknown genes that can cause hypertrophic nail dystrophy that is clinically indistinguishable from PC.

In conclusion, Clouston syndrome can have a variable presentation and can mimic other forms of hair and/or nail dysplasia. We recommend that any patient diagnosed with a type of PC with or without hair abnormalities be tested for the presence of connexin 30 mutations. This will help expand the spectrum of phenotypes associated with these mutations and may give some clues as to the cause of the phenotypic variability.

We would like to thank the patients for their participation in this study. Thanks to Andrew I. Cassidy Molecular Genetics Analysis Facility Department of Molecular and Cellular Pathology, Ninewells Medical School, Dundee, for DNA sequencing. W.H.I.M. and F.J.D.S. are funded by a Wellcome Trust Senior Research Fellowship (to W.H.I.M). M.A.M.vS. is supported by grants from ZON-MW (grant 920 03-085), Multigen BV, The Netherlands, and the Berliner Stiftung fur Dermatologie.

\section{REFERENCES}

Bowden PE, Haley JL, Kansky A, Rothnagel JA, Jones DO, Turner RJ: Mutation of a type II keratin gene (K6a) in pachyonychia congenita. Nat Genet 10:363-365, 1995

Calzavara-Pinton P, Carlino A, Benetti A, De Panfilis G: Pili torti and onychodysplasia. Report of a previously undescribed hidrotic ectodermal dysplasia. Dermatologica 182:184-187, 1991

Chang A, Lucker GP, van de Kerkhof PC, Steijlen PM: Pachyonychia congenita in the absence of other syndrome abnormalities. J Am Acad Dermatol 30:1017-1018, 1994 
Christianson AL, Fourie S: Family with autosomal dominant hidrotic ectodermal dysplasia: A previously unrecognised syndrome? Am J Med Genet 63:549-553, 1996

Copeland DD, Lamb WA, Klintworth GK: Calcification of basal ganglia and cerebellar roof nuclei in mentally defective patient with hidrotic ectodermal dysplasia. Analysis of intracranial concretions by electron microprobe. Neurology 27:1029-1033, 1977

Fraser FC, Der Kaloustian VM: A man, a syndrome, a gene: Clouston's hidrotic ectodermal dysplasia (HED). AmJ Med Genet 100:164-168, 2001

Hassed SJ, Kincannon JM, Arnold GL: Clouston syndrome: An ectodermal dysplasia without significant dental findings. Am J Med Genet 61:274-276, 1996

Jackson ADM, Lawler SD: Pachyonychia congenita: A report of six cases in one family. Ann Eugen 16:142-146, 1951

Jadassohn J, Lewandowsky F: Pachyonychia congenita. Berlin: Urban and Schwarzenberg, 1906

Kelsell DP, Di WL, Houseman MJ: Connexin mutations in skin disease and hearing loss. AmJ Hum Genet 68:559-568, 2001

Lamartine J, Munhoz Essenfelder G, Kibar Z, et al: Mutations in GJB6 cause hidrotic ectodermal dysplasia. Nat Genet 26:142-144, 2000

McLean WHI, Rugg EL, Lunny DP, et al: Keratin 16 and keratin 17 mutations caus pachyonychia congenita. Nat Genet 9:273-278, 1995

Pinheiro M, Freire-Maia N: Hair-nail dysplasia - a new pure autosomal dominant ectodermal dysplasia. Clin Genet 41:296-298, 1992

Rajagopalan K, Tay CH: Hidrotic ectodermal dysplasia: Study of a large Chinese pedigree. Arch Dermatol 113:481-485, 1977

Richard G: Connexin disorders of the skin. Adv Dermatol 17:243-277, 2001
Richard G: Human connexin disorders of the skin. Cell Commun Adhes 8:401-407, 2001b

Smith FJD, Jonkman MF, van Goor H, Coleman C, Covello SP, Uitto J, McLean WHI: A mutation in human keratin K6b produces a phenocopy of the K17 disorder pachyonychia congenita type 2. Hum Molec Genet 7:1143-1148, 1998

Smith FD, McKenna KE, Irvine AD, Bingham EA Coleman CM, Uitto J, McLean WHI: A mutation detection strategy for the human K6A gene and novel mutations in two cases of pachyonychia congenita type 1. Exp Dermatol 8:109-114,

Smith FJD, McKusick VA, Nielsen K, Pfendner E, Uitto J, McLean WHI: Cloning of multiple keratin 16 genes facilitates prenatal diagnosis of pachyonychia congenita type 1. Prenatal Diagnosis 19:941-946, 1999b

Smith FJD, Morley SM, McLean WHI: A novel connexin 30 mutation in Clouston syndrome. J Invest Dermatol 118:530-532, 2002

van Steensel MA, Smith FJD, Steijlen PM: A new type of pachyonychia congenita EurJ Dermatol 11:188-190, 2001

van Steensel MA, van Geel M, Steijlen PM: New syndrome of hypotrichosis, striate palmoplantar keratoderma, acro-osteolysis and periodontitis not due to mutations in cathepsin C. BrJ Dermatol 147:575-581, 2002

Templeton SF, Wiegand SE: Pachyonychia congenita-associated alopecia. A microscopic analysis using transverse section technique. Am J Dermatopathol 19: 180-184, 1997

Terrinoni A, Smith FJD, Didona B, et al: Novel and recurrent mutations in the genes encoding keratins K6a, K16 and K17 in 13 cases of pachyonychia congenita. J Invest Dermatol 117:1391-1396, 2001 
Chapter 7 


\title{
A Phenotype Resembling the Clouston Syndrome with Deafness Is Associated with a Novel Missense GJB2 Mutation
}

\author{
Maurice A. M. van Steensel, ${ }^{* 1}$ Peter M. Steijlen, ${ }^{*}$ Reno S. Bladergroen, ${ }^{* 1}$ Elisabeth H. Hoefsloot, $\dagger$ Connie M. \\ van Ravenswaaij-Arts, $\dagger$ and Michel van Geel*1 \\ *Department of Dermatology and †Department of Human Genetics, University Medical Centre Nijmegen, The Netherlands

\begin{abstract}
Mutations in GJB2 (connexin26) are associated with skin disorders and deafness. The Clouston syndrome (MIM129500) is associated with mutations in GJB6 (connexin30). Here, we describe a patient suffering from a Clouston-syndrome-like phenotype of thin hair, deafness, nail dystrophy, and mild erythrokeratoderma, caused by a novel spontaneous missense mutation in GJB2. The heterozygous mutation in codon 42, AAC >AAG, changes asparagine to lysine (N14K). Interestingly, this asparagine is near two of the residues mutated in Keratitis-like ichthyosis deafness (KID) syndrome (G12R and S17F), yet the phenotype associated with N14K strongly differs from the KID phenotype. Instead, there is clear phenotypic overlap with syndromes associated with connexin26 or 30 mutations. Our findings suggest that careful audiological evaluation of patients suffering from Cloustonsyndrome-like phenotypes is warranted and expand the spectrum of connexin26-associated disease.
\end{abstract}

Key words: Clouston syndrome/connexin26/gap junction/GJB2/hypotrichosis

J Invest Dermatol 291-293, 2004

Gap junctions are intercellular communication channels that are vital for cell growth and differentiation in various tissues. They consist of connexins, relatively small proteins that belong to an extensive protein family that exist throughout the vertebrate kingdom. During the past years, several often syndromic skin disorders have been shown to be associated with mutations in the skin-expressed connexin genes GJB2, GJB3, GJB4, and GJB6 (Richard, 2000; Kelsell et al, 2001). Sensorineural deafness is a frequent component of these disorders that otherwise have widely differing phenotypes. One of the most intriguing gap junction genes is GJB2 (connexin26, Cx26) that has been implicated in several different disorders such as non-syndromic sensorineural deafness, palmoplantar keratoderma with deafness, and keratitis (and hystrix-like) ichthyosis deafness (KID/HID) (Kelsell et al, 2001; van Geel et al, 2002; van Steensel et al, 2002). We now report a novel connexin 26 mutation that expands the spectrum of disorders associated with mutations in GJB2. The phenotype associated with this novel mutation resembles that of the Clouston syndrome (MIM129500), which is usually associated with mutations in GJB6 (Lamartine et al, 2000; Smith et al, 2002). Interestingly, the mutation affects an asparagine that neighbors amino acids associated with KID syndrome.

The patient, a 2-y-old girl, was born to non-consanguineous Dutch parents. There are no other sibs and the family history was wholly unremarkable. Shortly after birth, it

Abbreviation: KID, keratitis-like ichthyosis deafness

'Present address: Department of Dermatology, University Hospital Maastricht, PO Box 5800, 6202 AZ Maastricht, The Netherlands became clear that the child suffered from severe bilateral hearing loss that was later classified as sensorineural. Atopic eczema was said to have appeared directly after birth. Around age $6 \mathrm{mo}$, she fell ill and started refusing food. In addition, a slight developmental delay was noted at the time. At age 1, two episodes of viral enteritis led to dehydration, necessitating hospitalization twice. At two occasions during hospitalization, pronounced redness and swelling of the oral mucosa and gingiva were noted. The patient was referred to our department for diagnosis of her skin and nail problems.

Upon examination, we noted mild scalp hypotrichosis with lank, blonde hair. On the scalp some sharply demarcated erythematous plaques with some desquamation were present. In the neck we saw a papular exanthema but no other signs of atopic eczema. The patient displayed peculiar skin reactions upon application of brown adhesive bandaging (Fig 1a), consisting of sharply demarcated red plaques. The lesions resolved spontaneously with some scaling. Slight frontal bossing with seemingly deep-set eyes and 20-nail dystrophy were seen (Fig $1 b-c$, feet not shown). No other abnormalities were present except for mild perianal erythema. We could not substantiate the finding of excessive redness and swelling of the oral mucosa. The parents said that their daughter occasionally suffered from severe itching. Sweating was normal. The diagnosis presented some difficulty since the occurrence of nail dystrophy with hypotrichosis suggests the Clouston syndrome. Deafness, however, is not normally part of the Clouston syndrome phenotype. We considered the possibility of a novel variant of the Clouston syndrome, possibly associated with a GJB6 mutation and initiated connexin mutation screening. 


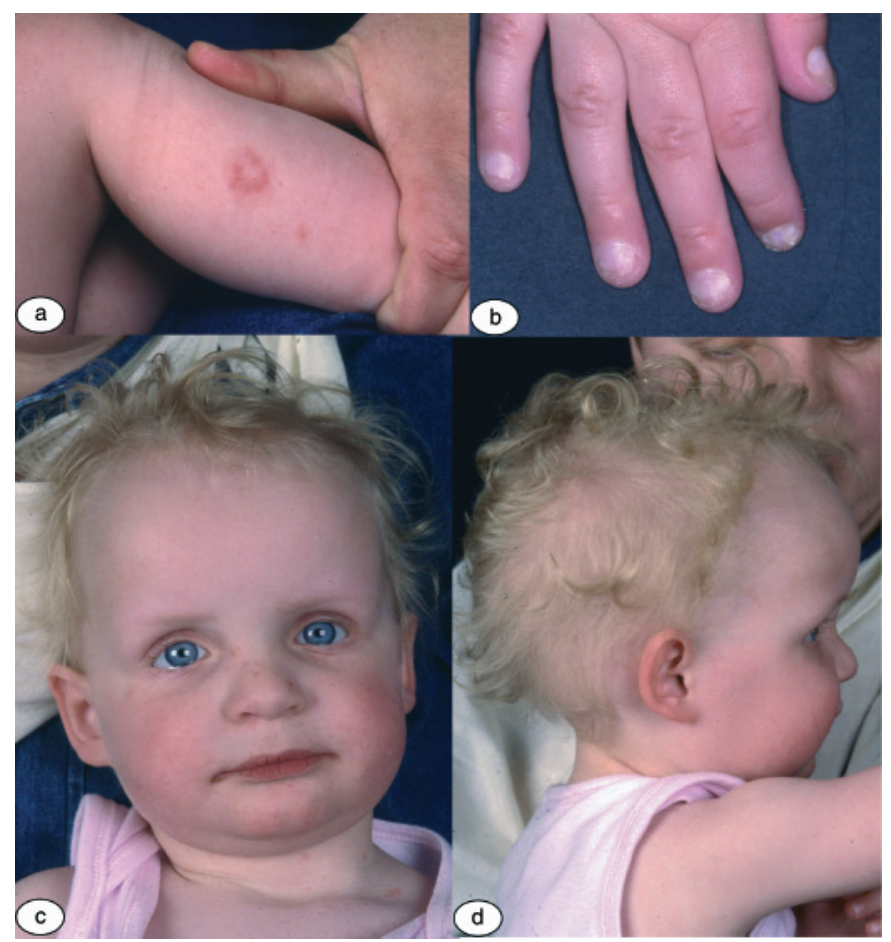

Figure 1

(a) Erythematous skin lesions arising after application of a brown adhesive bandage. (b) The fingernails are dystrophic. ( $c$ and $d$ ) The patient's phenotype. Note the frontal bossing in $(d)$ and seemingly deep-set eyes. The hair is thinly implanted.

Informed consent was obtained from the parents. DNA was isolated from peripheral blood lymphocytes using protocols described elsewhere (Miller et al, 1988). We amplified the coding regions of the skin-expressed connexin genes GJB2, GJB3, GJB4, GJB5, and GJB6 (connexin26, 31, 30.3, 31.1, and 30, respectively) by PCR. The PCR fragments were subjected to direct sequencing as previously described (van Steensel et al, 2002). The mutation analysis was repeated in an independent laboratory. Screening of 96 healthy controls for the nucleotide
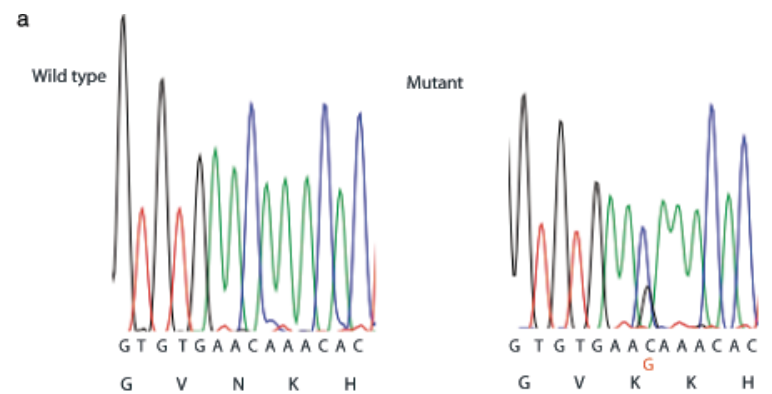

b

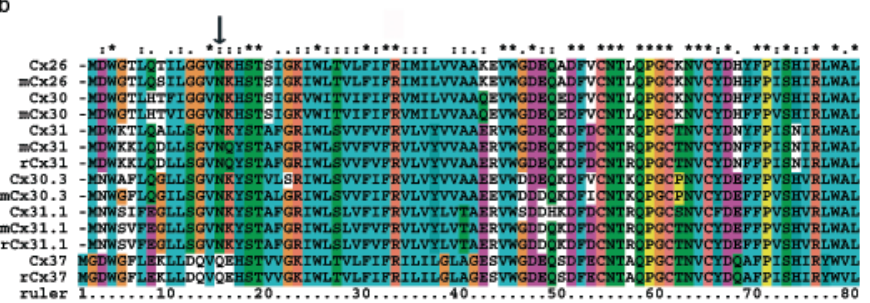

Figure 2

(a) Sequence traces showing the $44 \mathrm{C}>\mathrm{G}$ transversion mutation resulting in the substitution of asparagine 14 for a lysine. (b) CLUSTALX alignment of connexins from different mammalian species showing conservation of $\mathrm{N} 14$ ( $m=$ mouse, $r=$ rat, $h=$ human). 
variation was carried out by Mboll restriction analysis of the PCR products, where the mutated allele generates a site for digestion. In GJB3, GJB4, GJB5, and GJB6, no mutations or known polymorphisms were detected. In GJB2 (connexin26), we found a heterozygous $44 \mathrm{C}>\mathrm{G}$ transversion at codon 42), resulting in a substitution of an asparagine by a lysine (N14K, see Fig 2a). This asparagine is located at the intracellular $\mathrm{C}$-terminus of the protein and is conserved among connexins (Fig $2 b$ ). The mutation was not found in either parent or in 192 unrelated control alleles from the Dutch population (with Mboll restriction analysis). To our knowledge, this missense mutation has not been previously described in the literature.

Our results suggest that a phenotype resembling the Clouston syndrome but associated with deafness can be caused by a novel heterozygous mutation in GJB2. The Clouston syndrome has thus far only been associated with mutations in GJB6. Intriguingly, although most GJB2 mutations described to date are associated with unique phenotypes, the N14K substitution that we found leads to a phenotype that is remarkably similar to the Clouston syndrome. This may be explained by involvement of residue $\mathrm{N} 14$ in heterotypic connexon assembly. If mutated it may interfere with the incorporation of GJB6; however, the Clouston syndrome is not associated with absence of GJB6, therefore this hypothesis seems unlikely. Alternatively, some missense mutations in both connexins are known to lead to disturbed gap junction conductivity (Rabionet et al, 2000). Perhaps the N14K substitution has an effect on heterotypic connexon assembly and results in faulty gap junction function that is comparable with the Clouston syndrome-associated GJB6 mutations G11R and A88V. In addition, part of the phenotype may be explained by a trans-dominant effect of the GJB2 mutation on GJA1 and GJB6 expression (Rouan et al, 2001). The associated phenotype in our patient suggests that the effect of the Clouston syndrome GJB6 mutations is mediated at least in part by a similar mechanism. But neither trans-dominant effects nor disturbance of gap junction assembly and conductance can fully account for the phenotypes associated with GJB2 mutations. For instance the mutations in two different domains of the protein in KID/HID syndrome (G12R/S17F and D50N) and the patient described here (N14K) show that mutations in residues located close together can result in radically different phenotypes. KID/ HID syndrome is associated with severe bilateral earlyonset keratitis, severe erythrokeratoderma, hypotrichosis and a propensity to develop squamous cell carcinoma of the skin. Our patient on the other hand, suffering from a mutation of a residue close to those affected in KID/HID syndrome, has no keratitis, only mild erythrokeratoderma, mild hypotrichosis, and brittle nails. Her phenotype is actually more reminiscent of the Clouston syndrome. Explaining the obvious differences between the phenotypes is in our view difficult if we do not accept that at least some residues of GJB2 may have very specific roles in protein function that may not necessarily be related to gap junction assembly and function. Our results also point to an interesting difference between GJB2 and GJB6 with regard to their role in hearing loss. Although it was suggested that Cx26 and Cx30 form heteromeric connexons within the inner ear (Marziano et al, 2003), the Clouston syndrome is not associated with deafness. But a T5M mutation in GJB6 does cause dominant non-syndromic hearing loss (Grifa et al, 1999). The GJB2 mutations that are associated with skin symptoms then again all cause deafness. We have no explanation for this difference but as in the skin, transdominant effects on the expression of other connexins may be responsible for the hearing loss. GJB2 may have a more important role in gap junction assembly in the inner ear. Alternatively, GJB2 may have interactions with other proteins that are important for hair cell function that GJB6 lacks. Recent reports of an interaction with the transcription factor YAF-2 support this notion (Rodina et al, personal communication, 2003). Specifically, YAF2 protein could be co-immunoprecipitated with aa161-226 C-terminal fragment of Cx26 in vitro. Confocal microscopy revealed colocalization of YAF2 and Cx26 on the plasma membrane of HeLa cells stably co-transfected with those genes. This finding suggests protein-protein interaction between YAF2 and $\mathrm{Cx} 26$ in vivo.

Further studies, for example in vitro experiments using expression vectors bearing mutated connexin genes, are urgently needed to understand the underlying mechanism of the presently bewildering variety of connexin26-associated disorders.

This work is supported by grants from ZON-MW (920-03-085), the Berliner Stiftung für Dermatologie and the "Stichting Drie Lichten" foundation

DOI: 10.1111/j.0022-202X.2004.23204.x

Manuscript received October 1, 2003; revised December 3, 2003; accepted for publication January 5, 2004

Address correspondence to: Maurice A.M. van Steensel, MD, Department of Dermatology, University Hospital Maastricht, PO Box 5800, 6202 AZ Maastricht, The Netherlands. Email: mvst@sder.azm.nl

\section{References}

Grifa A, Wagner CA, D'Ambrosio L, et al: Mutations in GJB6 cause nonsyndromic autosomal dominant deafness at DFNA3 locus. Nat Genet 23:16-18,

Kelsell DP, Dunlop J, Hodgins MB: Human disease: Clues to cracking the connexin code? Trends Cell Biol 11:2-6, 2001

Lamartine J, Munhoz Essenfelder G, Kibar Z, et al: Mutations in GJB6 cause hidrotic ectodermal dysplasia. Nat Genet 26:142-144, 2000

Marziano NK, Casalotti SO, Portelli AE, Becker DL, Forge A: Mutations in the gene for connexin 26 (GJB2) that cause hearing loss have a dominan negative effect on connexin 30. Hum Mol Genet 12:805-812, 2003

Miller SA, Dykes DD, Polesky HF: A simple salting out procedure for extracting DNA from human nucleated cells. Nucleic Acids Res 16:1215, 1988

Rabionet R, Gasparini P, Estivill X: Molecular genetics of hearing impairment due to mutations in gap junction genes encoding beta connexins. Hum Mutat 16:190-202, 2000

Richard G: Connexins: A connection with the skin. Exp Dermatol 9:77-96, 2000

Rouan F, White TW, Brown N, et al: Trans-dominant inhibition of connexin- 43 by mutant connexin-26: Implications for dominant connexin disorders affecting epidermal differentiation. J Cell Sci 114:2105-2113, 2001

Smith FJ, Morley SM, McLean WH: A novel connexin 30 mutation in Clouston syndrome. J Invest Dermatol 118:530-532, 2002

van Geel M, van Steensel MA, Kuster W, Hennies HC, Happle R, Steijlen PM, Konig A: HID and KID syndromes are associated with the same connexin 26 mutation. Br J Dermatol 146:938-942, 2002

van Steensel MA, van Geel M, Nahuys M, Smitt JH, Steijlen PM: A novel connexin 26 mutation in a patient diagnosed with keratitis-ichthyosis-deafness syndrome. J Invest Dermatol 118:724-727, 2002 
Chapter 8 


\title{
Rapid Publication
}

\section{A 2-bp Deletion in the GJA1 Gene Is Associated With Oculo-Dento-Digital Dysplasia With Palmoplantar Keratoderma}

\author{
M.A.M. van Steensel, ${ }^{1 *}$ L. Spruijt, ${ }^{2}$ I. van der Burgt, ${ }^{2}$ R.S. Bladergroen, ${ }^{1}$ M. Vermeer, ${ }^{3}$ \\ P.M. Steijlen, ${ }^{1}$ and $M$. van Geel ${ }^{1}$ \\ ${ }^{1}$ Department of Dermatology, University Hospital Maastricht, Maastricht, The Netherlands \\ ${ }^{2}$ Department of Clinical Genetics, University Hospital Maastricht, Maastricht, The Netherlands \\ ${ }^{3}$ Department of Dermatology, University Medical Center Leiden, Leiden, The Netherlands
}

\begin{abstract}
Oculo-dento-digital dysplasia (ODDD, OMIM no. 164210) is a pleiotropic disorder characterized mainly by ocular anomalies, varying degrees of finger and toe syndactyly, and enamel defects. It is caused by missense mutations in the gene coding for the gap junction protein connexin 43 or GJA1. Other types of mutations have so far not been reported. Here we describe a Dutch kindred with reported. Here we describe a Dutch kindred with
ODDD showing a new symptom, palmoplantar keratoderma, and associated with a novel 2-bp deletion mutation of GJA1. The dinucleotide deletion 780781 delTG is located in the cytoplasmic C-terminal loop and leads to a frameshift. This is predicted to lead to the production of a slightly
truncated protein with 46 incorrect amino acids truncated protein with 46 incorrect amino acids
in the C-terminal cytoplasmic loop (C260fsX307). This novel mutation may explain the presence of skin symptoms. ๑ 2004 Wiley-Liss, Inc.
\end{abstract}

KEY WORDS: gap junction; connexin; keratoderma; skin; oculo-dento-digita syndrome

\section{INTRODUCTION}

Oculo-dento-digital dysplasia (ODDD, MIM no. 164210) is characterized by the presence of ocular anomalies, small nose with hypoplastic alae nasi, abnormal tooth enamel, and vary ing degrees of cutaneous syndactyly of fingers and toes. Skeletal abnormalities such as hypoplasia or aplasia of phalanges can also be present [Thomsen et al., 1998]. Neurological abnormalities such as leukodystrophic changes can be part of the phenotype [Gutmann et al., 1991; Loddenkemper et al. 2002]. Recently, missense mutations in the GJA1 gene that codes for the gap junction protein connexin 43 were shown to cause ODDD [Paznekas et al., 2003]. Apart from curly hair, skin abnormalities have not yet been described in association with ODDD [Adamski et al., 1994; Kjaer et al., 2004]. Thi seems difficult to understand considering the evident impor-

Grant sponsor: Pachyonychia Congenita Foundation; Grant sponsor: Barrier Therapeutics NV.

*Correspondence to: M.A.M. van Steensel, Department of Dermatology, University Hospital Maastricht, Maastricht, The Netherlands. E-mail: mvst@sder.azm.n

Received 11 May 2004; Accepted 29 July 2004

DOI 10.1002/ajmg.a.30412

(c) 2004 Wiley-Liss, Inc. tance of GJA for skin gap junctions and its evident involvement in gap junction skin disease [Rouan et al., 2001]. Here, we report on a family having ODDD with skin symptoms, caused by a novel deletion mutation in GJA1. The consequences of the mutation may offer an explanation for the presence of skin symptoms in this family.

\section{CLINICAL REPORT}

A 36-year-old woman of Dutch descent was referred to our department for diagnosis of a syndrome of hyperkeratosis of the palms and soles with several other abnormalities. Hair growth was said to have always been sparse. She had syndactyly of fingers and toes and had frequent caries, resulting in the removal of several teeth. A pediatrician who examined her daughter for growth retardation had previously noted hypotelorism. The left eye was hypermetropic, while the right one was myopic. More detailed ophthalmological examination was never performed because the patient had no other complaints. CT or MRI imaging of the brain had never been performed either, also because neurological complaints had never been present. Her 4-year-old son and 2-year-old daughter also had varying degrees of syndactyly and facial features similar to their mother. The son had hyperkeratosis of the soles and palms though to a lesser degree than his mother. He had bilateral myopia and had had eczema in early childhood. The daughter's hair was sparse and grew slowly. The maternal grandmother was said to have died from multiple sclerosis and had syndactyly of the toes. The maternal great-grandfather was said to have had a syndactyly as well. No mention was made of their having special facial features and photographs were not available.

Upon examination of the proposita, we noted a distinctive facial phenotype with hypoplasia of the alae nasi, short palpebral fissures, prominent cheekbones, and freckling (Fig. 1). The eyebrows were white and sparse and the eyes a pal llings and showed a brownish discoloration indicative of enamel hypoplasia. Two molars were missing. The hair appeared normal but was curly. Axillary hair was absent. On the hands she had short fifth digits bilaterally, the shortening being in the middle phalanx with a pronounced camptodactyly. There was no syndactyly of the fingers. The nails were thin. The palms showed a diffuse yellow-orange hyperkeratosis (Fig. 2a). On the feet, there was bilateral syndactyly of the second and third toes (Fig. 2b). The soles showed diffuse yellow hyperkeratosis with a transgredient erythema bordering it. Biopsy of the skin showed a non-epidermolytic hyperkeratosis. Both children were available for examination.

The son wore glasses for his myopia and had pale blue eyes with thin blonde hair. He had hypotelorism with thin alae nasi as well as short palpebral fissures with epicanthic folds. The 
van Steensel et al.

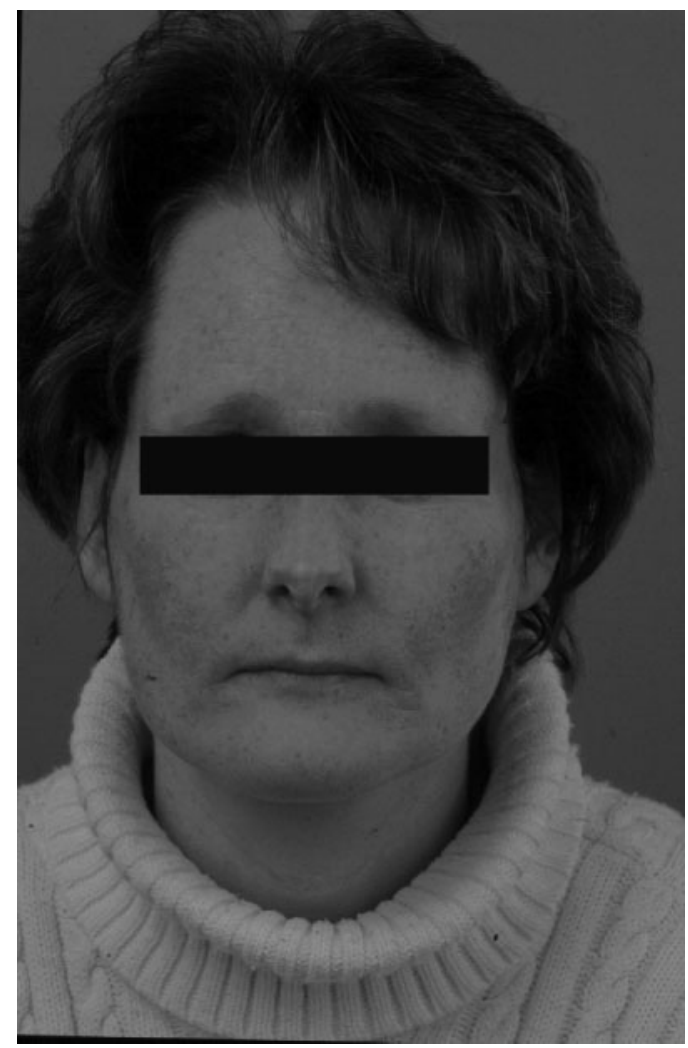

Fig. 1. Facial phenotype of the proposita. Note hypoplasia of the alae

scalp hair was rigid and short. Some brown discoloration was evident on the front teeth. On both hands, the second phalanx of the fifth digit was shortened and bowed giving rise to camptodactyly (Fig. 3a). The nails were mildly dystrophic. Light hyperkeratosis was noted. On the feet, the hyperkeratosis was more pronounced. A syndactyly of the second, third, and fourth toes was present bilaterally.

The daughter likewise presented short palpebral fissures with epicanthic folds and a low nasal bridge. The teeth appeared normal. The skin was pale and the irides were a striking pale blue. The scalp hair was sparse (Fig. $3 \mathrm{~b}$ ). On the hands she had bilateral clinodactyly V. Both feet showed cutaneous syndactyly of digits II-IV.

The patient and her children had a normal intelligence. Neurological abnormalities were not noted. A skeletal survey of the mother had been performed but was not available to us. The radiological report stated that the middle phalanx of the second, third, and fourth toes was missing bilaterally. Based on the clinical abnormalities we diagnosed our patients with oculo-dento-digital syndrome.

\section{MUTATION ANALYSIS}

Informed consent was obtained from the patient and she assented to having her children examined as well. DNA was
A

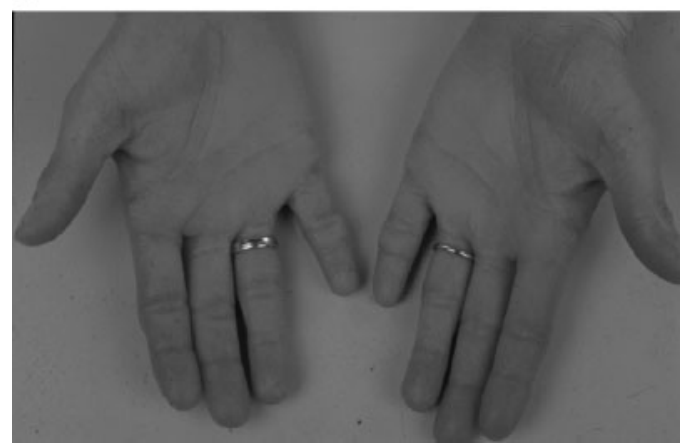

B

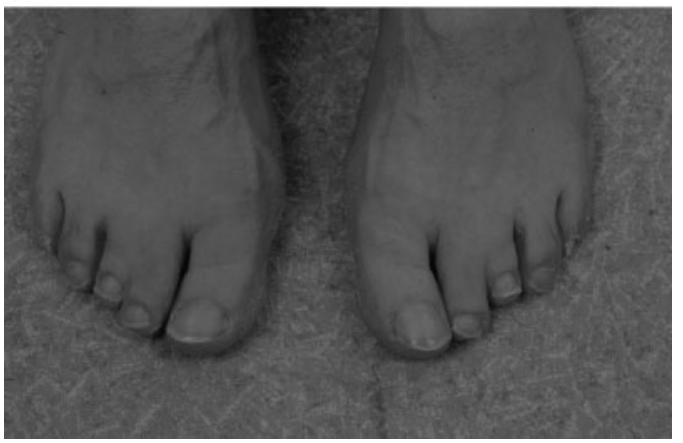

Fig. 2. A: The propsita's hands. Digit V on both hands is shortened due to hypoplasia of the middle phalanx. B: The proposita's feet with cutaneous syndactyly of the 2 nd and 3 rd toes.

isolated from peripheral blood leucocytes using methods described elsewhere [van Steensel et al., 2002].

The GJA1 gene was amplified with PCR using primers Cx43F 5'-TGGGACAGGAAGAGTTTGCAC-3' and Cx43R 5'CACCTGGTGCACTTTCTACAGCAC- $3^{\prime}$. GJA1 has a pseudogene, hence the forward and reverse primers were designed to be in the GJA1 intron and in the $3^{\prime}$ UTR, respectively. The gene was analyzed using PCR primers and the sequencing primers Cx43FS 5'-GGTGGCCTTCTTGCTGATCC-3', Cx43RS 5'-TGGGCAGGGATCTCTTTTGC-3', and Cx43FS2 5'-GGTTGCCCAAACTGATGGTG-3', using the BigDyeDeoxy terminator system and an ABI 3100 capillary sequencer (ABI). In the patient and in her children we found a dinucleotide deletion (Fig. 4). This mutation is predicted to lead to a frameshift and a premature stop. As the mutation generates a $P f l \mathrm{MI}$ restriction site, we used restriction analysis to screen 100 unrelated controls in which we did not find the mutation.

\section{DISCUSSION}

Here, we describe a family having ODDD (MIM no. 164210) with palmoplantar keratoderma and caused by a previously undescribed mutation. The reading frame that results from the deletion remains intact over 138 nucleotides, allowing for the incorporation of 46 amino acids before premature termination. 
A 2-bp Deletion in the GJA1 Gene

A

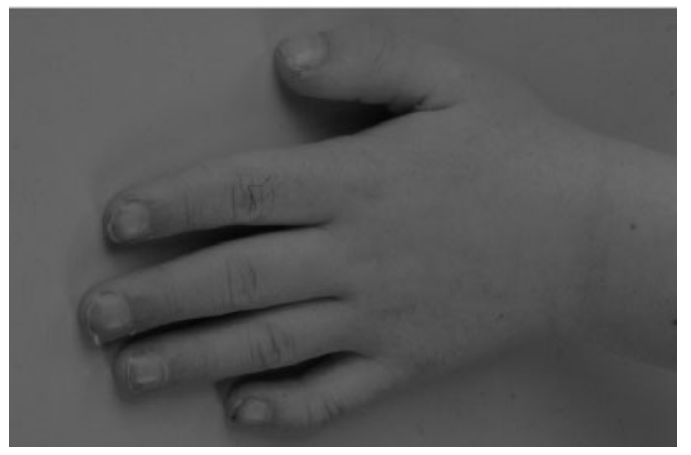

B

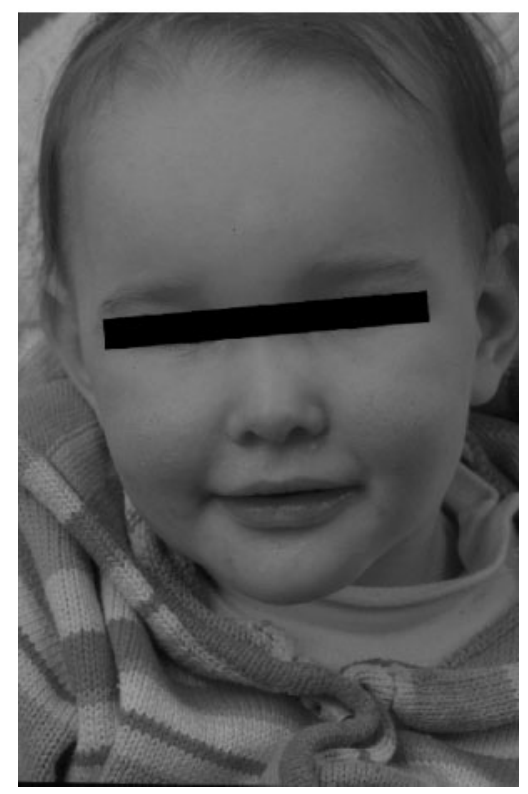

Fig. 3. A: The son's left hand with pronounced hypoplasia of the middle phalanx of digit $\mathrm{V}$ and camptodactyly. B: The daughter's facial phenotype.

As the sequence does not correspond to any known functional protein as determined by PBLAST, it can be expected to disturb the conformation of the now shortened C-terminal part of GJA1. Almost all mutations associated with ODDD found so far have been in the N-terminus or early first transmembrane domain, except for one codon duplication that affects the first extracellular loop (E1) and a substitution that also affects E1 in a phenotype of ODDD with curly hair [Paznekas et al., 2003; Kjaer et al., 2004]. To our knowledge, this is the first reported mutation affecting the C-terminal cytoplasmic loop.

The family history is notable for the maternal grandmother who was said to have died from multiple sclerosis. Since she

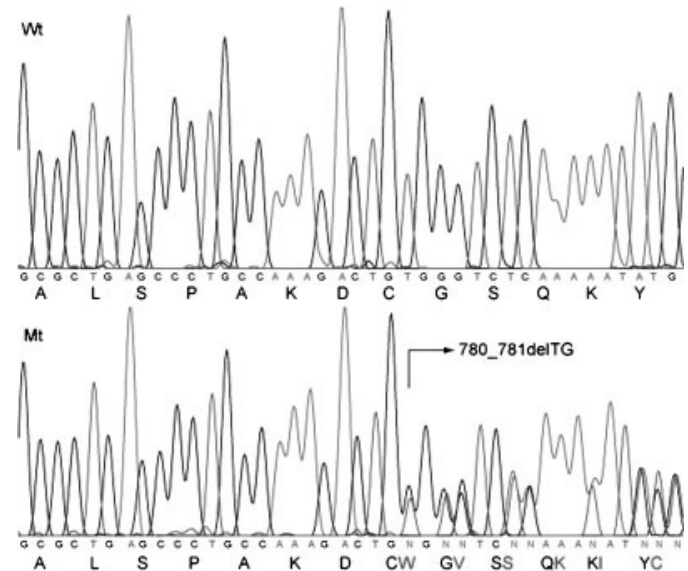

Fig. 4. Sequence traces showing a 2-bp deletion in GJA1.

also had syndactyly of toes, it is tempting to speculate that she in fact suffered from the progressive neurological symptoms that are occasionally seen in ODDD and can include spastic paraplegia, mimicking some of the symptoms of multiple sclerosis [Loddenkemper et al., 2002]. Absence of middle phalanges has to our knowledge not been previously described, although hypoplasia seems to be a common finding. Like 4-5 syndactyly, the shortening of middle phalanges seems to be a variable finding and extreme shortening to the point of absence would certainly seem to be possible in this regard.

While a chance association with the skin symptom is possible, we felt that the presence of the symptom in both mother and son argues against that possibility. Also, the strong and widespread expression of GJA1 in the skin [Tada and and widespread expression of GJA1 in the skin [Tada and
Hashimoto, 1997] is consistent with such a finding. Why skin symptoms are not found in ODDD caused by missense mutations is not clear. It is currently thought that in ODDD caused by missense mutations, connexin 31 (GJB5) can compensate, having the same expression pattern as GJA1 (Hodgins M, personal communication, 2004). Functional analyses of these mutations have not been performed however. It is known that mutations have not been performed however. It is known that
skin disease-causing mutations in the gap junction proteins GJB2 and GJB6 exert their effect partly through reduction of GJA1 function [Rouan et al., 2001]. Therefore, it seems possible that the skin symptoms in our patients are the consequence of a severe reduction of the contribution of GJA1 to gap junctions. Indeed, preliminary analysis of the mutant demonstrates a pronounced transport defect, with both wild type and mutant GJA1 showing perinuclear accumulation (not shown). Cell membrane staining is almost absent. The total amount of GJA1 that is detectable by immunofluoresence in patient skin seems to be severely reduced. It is not yet clear whether this is the lasult of redur. The known deler lational effect. The known deleterious effect of mutations in
other gap junction proteins on the assembly of gap junction hemichannels in the Golgi apparatus [Evans et al., 1999; Martin et al., 2000, 2001; Marziano et al., 2003] supports the latter hypothesis. Further studies will be needed to confirm it. These should also show a less pronounced effect on trafficking of i should al 1 show a of missense GJA1 mutations that do not cause skin symptoms. It will be of interest to examine the consequences of other
ODDD mutations. As these are in the transmembrane and E1 
van Steensel et al.

domains, they may have quite different effects on transport, perhaps explaining the usual absence of skin symptoms in ODDD.

\section{REFERENCES}

Adamski H, Chevrant-Breton J, Odent S, Patoux-Pibouin M, Le Marec B, Laudren A, Urvoy M. 1994. Hair dysplasia in oculo-dento-digital syndrome. Apropos of a mother-daughter case. Ann Dermatol Venereol 121

Evans WH, Ahmad S, Diez J, George CH, Kendall JM, Martin PE. 1999. Trafficking pathways leading to the formation of gap junctions. Novartis Found Symp 219:44-54.

Gutmann DH, Zackai EH, McDonald-McGinn DM, Fischbeck KH, Kamholz J. 1991. Oculodentodigital dysplasia syndrome associated with abnormal cerebral white matter. Am J Med Genet 41:18-20.

Kjaer KW, Hansen L, Eiberg H, Leicht P, Opitz JM, Tommerup N. 2004 Novel Connexin 43 (GJA1) mutation causes oculo-dento-digital dysplaNovel Connexin 43 (GJA1) mutation causes oculo-den
sia with curly hair. Am J Med Genet 127A:152-157.

Loddenkemper T, Grote K, Evers S, Oelerich M, Stogbauer F. 2002. Neurological manifestations of the oculodentodigital dysplasia syndrome. J Neurol 249:584-595.

Martin PE, Mambetisaeva ET, Archer DA, George CH, Evans WH. 2000. Analysis of gap junction assembly using mutated connexins detected in Charcot-Marie-Tooth X-linked disease. J Neurochem 74:711-720.

Martin PE, Errington RJ, Evans WH. 2001. Gap junction assembly: Multiple connexin fluorophores identify complex trafficking pathways.

Marziano NK, Casalotti SO, Portelli AE, Becker DL, Forge A. 2003. Mutations in the gene for connexin 26 (GJB2) that cause hearing loss have a dominant negative effect on connexin 30 . Hum Mol Genet 12:805-812.

Paznekas WA, Boyadjiev SA, Shapiro RE, Daniels O, Wollnik B, Keegan CE Innis JW, Dinulos MB, Christian C, Hannibal MC, Jabs EW. 2003. Connexin 43 (GJA1) mutations cause the pleiotropic phenotype of oculodentodigital dysplasia. Am J Hum Genet 72:408-418.

Rouan F, White TW, Brown N, Taylor AM, Lucke TW, Paul DL, Munro CS, Uitto J, Hodgins MB, Richard G. 2001. Trans-dominant inhibition of connexin-43 by mutant connexin-26: Implications for dominant con2113.

Tada J, Hashimoto K. 1997. Ultrastructural localization of gap junction protein connexin 43 in normal human skin, basal cell carcinoma, and protein connexin 43 in normal human skin, basal cell
squamous cell carcinoma. J Cutan Pathol 24:628-635.

Thomsen M, Schneider U, Weber M, Niethard FU. 1998. The different appearance of the oculodentodigital dysplasia syndrome. J Pediat Orthop B 7:23-26. van Steensel MA, van Geel M, Nahuys M, Smitt JH, Steijlen PM. 2002.
A novel connexin 26 mutation in a patient diagnosed with keratitisichthyosis-deafness syndrome. J Invest Dermatol 118:724-727. 
Chapter 9 


\section{Does Recessive EKV Exist?}

To the Editor:

We read the article by Terrinoni et al in the March issue of the JID with much interest. In it, they described their finding of a putative recessive GJB3 (connexin 31) mutation associated with erythrokeratodermia variabilis (EKV). The existence of recessive skin disease-associated connexin mutations would contribute significantly to our understanding of gap junction biology. But we have a number of concerns with the current report, that in our view, need to be addressed before the existence of recessive EKV can be accepted.

All data that are currently available with regard to gap junction gene mutations associated with skin disease sug gest that the skin symptoms are caused by (trans-)dominant effects of the mutations. Almost all skin disease-associated mutations interfere with the transport of the mutant protein. The mutant protein will, however, be incorporated into a gap junction in the presence of a wild-type homotypic or heterotypic gap junction protein (Thomas et al, 2004). For GJB2, mutations such as $\mathrm{D} 66 \mathrm{H}$, which is associated with Vohwinkel's syndrome, have been shown to interfere in a trans-dominant manner with the expression of GJA1 (Rouan et al, 2001; Thomas et al, 2004) and can lead to increased sensitivity to apoptosis of keratinocytes (Bakirtzis et al, 2003). The dominant mutations in GJB3 that cause EKV have been shown to be gain-of-function mutants that lead to increased sensitivity to apoptosis of keratinocytes or interfere with gap junction conductance (Di et al, 2002; Common et al, 2003; Rouan et al, 2003). Dominant mutations in GJB6 can interfere with transport of the protein and impair the assembly of heteromeric GJB6-containing gap junctions (Common et al, 2002). At least one of the mutations associated with KID syndrome, D50N, leads to a gain of function of the gap junction (D. Gonzalez, personal communication, 2003). Dominant mutations associated with hearing loss seem to traffic to the cell membrane but impair channel function once inserted (Common et al, 2002; Marziano et al, 2003). They do not modify channel composition or lead to a gain-of-function in contrast to the mutations associated with skin disease. Recessive mutations associated with hearing loss have been found mostly in GJB2, suggesting that recessive missense or nonsense mutations in other connexins that are expressed in the ear need not have functional significance there with the possible exception of GJA1 in which recessive mutations have been found in deaf patients (Liu et al, 2001). These findings, however, have so far not been replicated. The same observation may very well apply to the skin. There is a previous report that describes a

Abbreviation: EKV, erythrokeratodermia variabilis recessive mutation in GJB3, L34P, and claims that it causes EKV (Gottfried et al, 2002). The clinical data supplied are scarce and, in our view, do not support the diagnosis of EKV. Moreover, the paper shows that the mutant protein is sequestered in the cytoplasm and does not contribute to gap junction assembly. Functional studies showing alteration of gap junction function were not performed. As stated above, incorporation into a gap junction and alteration of its function or, alternatively, interference with the composition of the gap junction seem to be required for causation of a skin phenotype. Also, the absence of a gap junction protein does not necessarily have functional consequences. We have shown that connexin 30.3 (GJB4) is not required for skin function at all. Dominant point mutations in GJB4 cause EKV but homozygosity for a 4 bp-deletion in GJB4 appears to be a polymorphism, at least in the Dutch population (Macari et al, 2000; Van Geel et al, 2002). The polymorphism is rare, underscoring the need for screening of a sufficient number of controls. We note that Terrinoni et al do not mention the number of controls used to exclude E100K as a polymorphism. Finally, conservation of residues in gap junctions is not a guarantee for their functional importance. The conserved arginine at position 32 in GJB4 can apparently be substituted by a tryptophan without adverse consequences, as this change has been demonstrated to be a polymorphism in Spanish and Dutch populations (Lopez-Bigas et al, 2001; Van Geel et al, 2002). It was also demonstrated that this polymorphism does not lead to functional impairment of gap junctions (Rouan et al, 2003). In conclusion, the available functional data suggest that gap junction mutants that cause skin disease seem to be causing symptoms through a gain-of-function mechanism that requires incorporation of the mutant into a gap junction.

Another concern is with the correctness of the clinical diagnosis. The photographs accompanying the paper show abnormalities that in our view could also be consistent with the diagnosis of cyclic hyperkeratosis with ichthyosis as described by Sybert et al (1999). A hallmark feature of this particular disorder is an epidermolytic hyperkeratosis. Looking at the histology depicted in Fig $1 c$ of the paper, there is a definite indication of epidermolysis there, particularly in the suprabasal layer, where cytolysis of keratinocytes also seems to be present. This may also be an artifact, but other features of EKV such as elongation of rete ridges seem to be absent. There seems to be some hypergranularity of the granular layer but at the magnification and quality provided it is not possible to discern whether actual clumping of tonofilaments is present. Electron microscopy would be helpful in this regard. The clinical appearance of cyclic hyperkeratosis is also quite similar to that of EKV as evident from Fig 3 in the Sybert et al paper. This keratin 


\section{LETTER TO THE EDITOR}

disease leads to migrating, erythematous, and sharply demarcated plaques that mimic EKV. It would be important to know whether Terrinoni's patient had any blistering shortly after birth as this symptom can distinguish the two disorders. As it is, we are not convinced that the diagnosis EKV would be the correct one in the patient described by Terrinoni et al. The diagnosis of cyclic ichthyosis with epidermolytic hyperkeratosis seems more likely. We suggest that the present case of EKV be tested for KRT1 mutations. It is conceivable that testing of cases of apparent EKV without mutations in GJB3 or 4 will reveal several KRT1 mutations.

A recessive mutation associated with a skin disease would be a significant finding. We feel that the present case report raises sufficient concerns to suggest that the further confirmatory studies are required. First of all, we would suggest that a KRT1 mutation be ruled out. Second, if the diagnosis of EKV can be maintained, the E100K mutation needs to be confirmed as such by testing an adequate number of controls and with functional studies. It would be of interest to examine whether the mutation influences the expression or transport of other skin-expressed connexins in any way and impairs gap junction functionality if inserted into the membrane. Until these concerns are addressed, we feel that there is insufficient evidence to accept the existence of recessive EKV caused by a homozygous E100K substitution.

Maurice A. M. van Steensel and Michel van Gee Department of Dermatology, University Hospita Maastricht, Maastricht, The Netherlands

DOI: 10.1111/j.0022-202X.2004.23517.x

Manuscript received April 23, 2004; revised July 12, 2004; accepted for publication July 20, 2004

Address correspondence to: Maurice A. M. van Steensel, MD, Dermatology Department, University Hospital Maastricht, PO Box 5800, 6202 AZ Maastricht, The Netherlands. Email: mvst@sder.azm.nl
THE JOURNAL OF INVESTIGATIVE DERMATOLOGY

\section{References}

Bakirtzis G, Choudhry R, Aasen T, et al: Targeted epidermal expression of mutan Connexin 26(D66H) mimics true Vohwinkel syndrome and provides a model for the pathogenesis of dominant connexin disorders. Hum Mol Genet 12:1737-1744, 2003

Common JE, Becker D, Di WL, Leigh IM, O'Toole EA, Kelsell DP: Functional studies of human skin disease- and deafness-associated connexin 30 mutations. Biochem Biophys Res Commun 298:651-656, 2002

Common JE, Di WL, Davies D, Galvin H, Leigh IM, O'Toole EA, Kelsell DP: Cellular mechanisms of mutant connexins in skin disease and hearing loss. Cell Commun Adhes 10:347-351, 2003

Di WL, Monypenny J, Common JE, et al: Defective trafficking and cell death is characteristic of skin disease-associated connexin 31 mutations. Hum Mol Genet 11:2005-2014, 2002

Gottfried I, Landau M, Glaser F, et al: A mutation in GJB3 is associated with recessive erythrokeratodermia variabilis (EKV) and leads to defective trafficking of the connexin 31 protein. Hum Mol Genet 11:1311-1316, 2002

Liu XZ, Xia XJ, Adams J, et al: Mutations in GJA1 (connexin 43) are associated with non-syndromic autosomal recessive deafness. Hum Mol Genet 10: 2945-2951, 2001

Lopez-Bigas N, Rabionet R, Arbones ML, Estivill X: R32W variant in Connexin 31: Mutation or polymorphism for deafness and skin disease? Eur $\mathrm{J}$ Hum Genet 9:70, 2001

Macari F, Landau M, Cousin P, et al: Mutation in the gene for connexin 30.3 in a family with erythrokeratodermia variabilis. Am J Hum Genet 67 1296-1301, 2000

Marziano NK, Casalotti SO, Portelli AE, Becker DL, Forge A: Mutations in the gene for connexin 26 (GJB2) that cause hearing loss have a dominant negative effect on connexin 30. Hum Mol Genet 12:805-812, 2003

Rouan F, Lo CW, Fertala A, et al: Divergent effects of two sequence variants of GJB3 (G12D and R32W) on the function of connexin 31 in vitro. Exp Dermatol 12:191-197, 2003

Rouan F, White TW, Brown N, et al: Trans-dominant inhibition of connexin-43 by mutant connexin-26: Implications for dominant connexin disorders affecting epidermal differentiation. J Cell Sci 114:2105-2113, 2001

Sybert VP, Francis JS, Corden LD, Smith LT, Weaver M, Stephens K, McLean WH: Cyclic ichthyosis with epidermolytic hyperkeratosis: A phenotype conferred by mutations in the $2 B$ domain of keratin K1. Am J Hum Genet 64 : 732-738, 1999

Thomas T, Telford D, Laird DW: Functional domain mapping and selective transdominant effects exhibited by Cx26 disease-causing mutations. J Biol Chem 279:19157-19168, 2004

Van Geel M, Van Steensel MA, Steijlen PM: Connexin 30.3 (GJB4) is not required for normal skin function in humans. Br J Dermatol 147:1275-1277, 2002 
Chapter 10 


\section{Cryptic Splicing at a Non-Consensus Splice-Donor in a Patient with a Novel Mutation in the Plakophilin-1 Gene}

\author{
To the Editor:
}

Desmosomes are intercellular junctions that serve to anchor intermediate filaments in one cell to neighboring cells, creating a supercellular scaffold imparting mechanical strength to cells and tissue. The desmosomal complex contains members of the desmosomal cadherin, the plakin and the armadillo repeat protein families (McMillan and Shimizu, 2001). Plakophilin-1 (PKP1) is an armadillo protein, mainly concentrated in the suprabasal layers of the epidermis (Schmidt et al, 1997). PKP1 may interact either directly with intermediate filaments or indirectly through plakoglobin and desmoplakin (Kowalczyk et al, 1999), hence playing a crucial role in the maintenance of desmosome-intermediate filament interactions. This role is illustrated by the human phenotype caused by homozygous null and splice site mutations in plakophilin-1, ectodermal dysplasia-skin fragility (McGrath) syndrome (McGrath et al, 1997, 1999; Whittock et al, 2000; Hamada et al, 2002). The syndrome is characterized by skin fragility, disabling cracking palmoplantar keratoderma, sub-total hypotrichosis, and nail dystrophy. The skin shows suprabasal thickening of the epidermis and loss of cell cohesion in the intermediate layers of the epidermis (acantholysis) Nearly all patients reported so far were severely affected children suffering from almost complete hair loss and extensive skin erosions induced by light trauma. Reduced sweating was also reported (McGrath et al, 1997). Hamada et al (2002) recently reported a patient with a somewhat milder phenotype of relatively few, late-onset skin erosions, late-onset palmoplantar keratoderma, and alopecia. In this patient, low residual expression of full-length PKP1 apparently reduced the severity of his disease. Here we report a patient whose phenotype is even milder and describe the molecular mechanisms that may contribute to it. Informed consent was obtained from the patient, Ethical Committee approval according to the Helsinki guidelines was obtained prior to this investigation.

A 33-y-old Dutch male suffered, since birth, from vulnerability of the skin and recurrent cutaneous infections. Ever since childhood he complained of extreme plantar hyperkeratosis that hampered walking. No living relatives could be located as the patient did not know his parents. He presented with sharply demarcated, oozing erythematous lesions predominantly on the buttocks and in the inguinal area (Fig 1a). The lesions were induced by shearing trauma

Abbreviation: PKP1, plakophilin-1 and often showed secondary infection. There was no blistering. The scalp hair was dark, thick and curly and could easily be plucked without pain (Fig 1b). Eyebrows, eyelashes, pubic and axillary hair were sparse. Marked cracking hyperkeratosis was present on the soles of the feet (Fig 1c). All toenails and some of the fingernails were thickened and showed subungual hyperkeratosis. The palms displayed circumscribed hyperkeratoses. In addition, follicular hyperkeratoses were found on the thorax. Ophthalmologic examination showed stellate opacities of both lenses. The teeth were normal and sweating was not impaired. Histological examination of three biopsies obtained from erosive lesions of the axillary and inguinal region consistently displayed suprabasal splitting and acantholysis. Acantholysis was also observed within the hair follicles of the scalp. Electron microscopic examination of a skin biopsy obtained from the elbow demonstrated clumping of tonofilaments in keratinocytes (not shown).

We diagnosed the patient with ectodermal dysplasia-skin fragility (McGrath) syndrome, and sequenced the coding regions and exon boundaries of the plakophilin-1 gene on genomic DNA to confirm the diagnosis (PCR primer sequences and conditions available on request). A homozygous $G$ to $A$ transition in the splice donor site of exon 9 $(1680+1 \mathrm{G}>\mathrm{A}$, IVS9 $+1 \mathrm{G}>\mathrm{A})$ was identified that putatively abolishes the splice donor site (Fig 2a) and an Xcml restriction site. The mutation was not detected by restriction analysis in 168 control alleles from the Dutch population. No other PKP1 nucleotide variations were detected in the patient. Transcriptional analysis of RNA isolated from a skin biopsy (RNEasy kit, Qiagen, Hiden, Germany) by RT-PCR (Superscript first-strand synthesis system, Invitrogen, Bre$\mathrm{da}$, the Netherlands) and subsequent sequencing (BigDyeDeoxy Terminator kit, Applied Biosystems, Foster City, CA) identified transcripts with retention of intron 9 , besides a smaller alternative-splicing product (Fig 2b). The latter product results from splicing at a non-consensus (GC) cryptic splice donor site within exon 9, allowing in-frame splicing to exon 10 with the loss of 45 nucleotides from the $3^{\prime}$ end of exon 9 (Fig $\left.2 b, c\right)$. The predicted protein product lacks 15 amino acids in the sixth armadillo repeat, but will otherwise be intact as a $\pm 78.5 \mathrm{kDa}$ product (Fig $2 \mathrm{c}$ ). Immunohistochemical staining of skin biopsies using a monoclonal antibody directed against plakophilin-1 confirmed protein expression. Punctate PKP1 staining throughout most layers of the epidermis was seen (Fig 1d). Expression seemed to be strongest in the suprabasal layers. In healthy individuals, the expression pattern was essentially identical (Fig 1d). Using Western blotting 


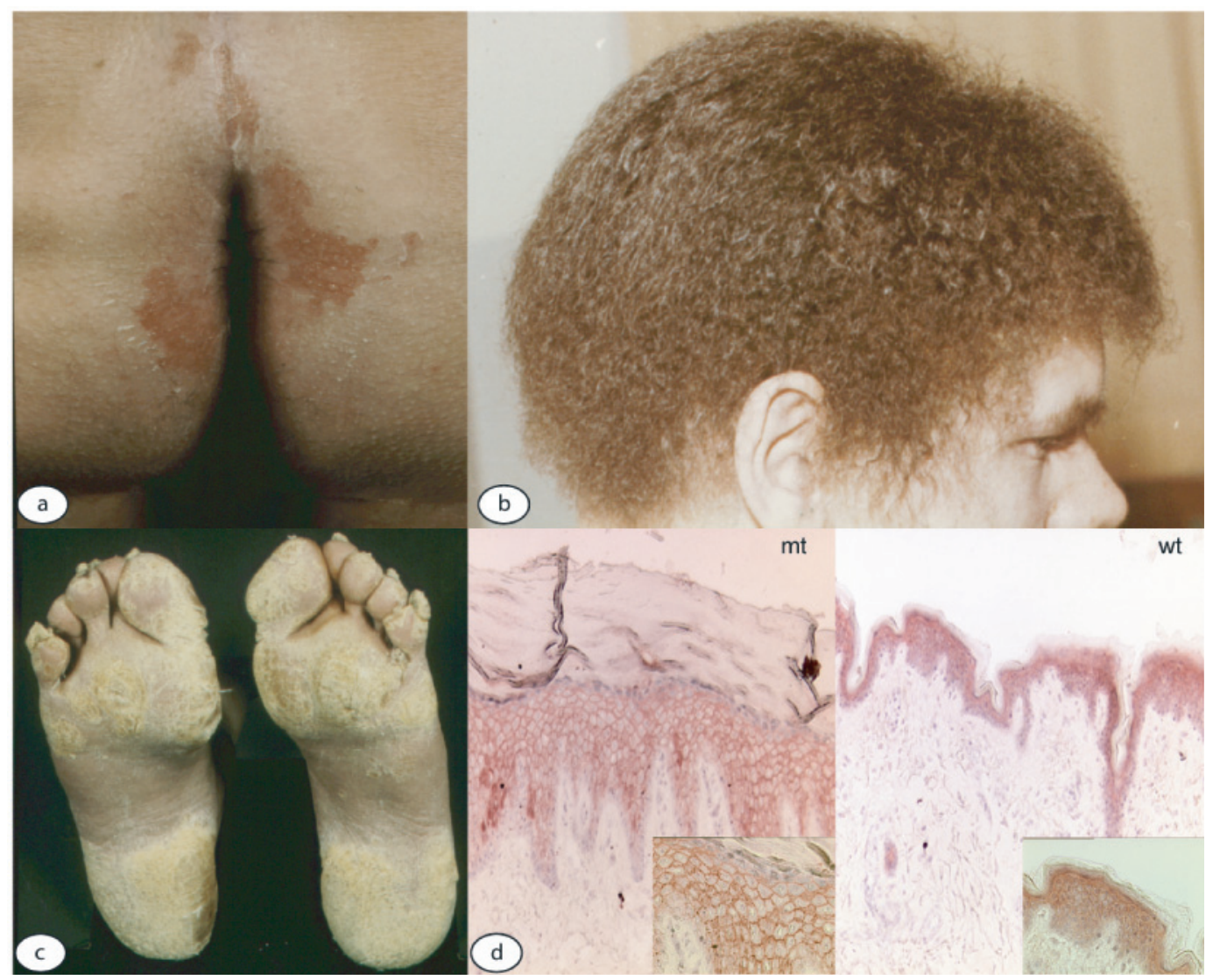

Figure 1

Phenotype of the patient and immunohistochemical analysis of the skin. (a) Erosive skin lesions on the buttocks. (b) Relatively normal amount of scalp hair; note the woolly hair. (c) Pronounced plantar hyperkeratosis with fissures and nail dystrophy. (d) Immunohistochemical analysis of PKP-1 expression in patient skin (mt) and in normal skin (wt). The patient skin shows pronounced hyperkeratosis and acanthosis with punctate PKP-1 staining throughout the epidermis $(\times 5)$. Normal skin shows a similar membrane staining $(\times 5)$. Insets: $\times 20$ enlargement. Slides were subjected to a standard peroxidase immunostaining procedure with primary mouse lgG1 anti-PKP1 monoclonal antibody (Clone PP1-5C2, Progen Biotechnik GmBH, Heidelberg, Germany) and VECTASTAIN ABC reagent (Vector Laboratories, Burlingame, CA).

(NuPAGE high-performance gel and blotting system, Invitrogen) we were able to confirm expression of the truncated protein (data not shown).

These results suggest that the relatively mild phenotype observed in our patient is the result of a partial PKP1 protein "rescue". A cryptic splice donor site drives in-frame splicing of approximately half of exon 9 to exon 10 , consequently allowing expression of a shortened but partially functional protein. Immunohistochemical examination of skin biopsies in our patient confirms the presence of the protein. The phenotype may be explained by either insufficiency of expressed shortened protein, or the armadillo repeat deletion may interfere with the interactions between plakophilin-1 and its partner molecules.

Use of cryptic (alternative) GC splice sites has been observed to account for $0.5 \%-1 \%$ of instances of splicing in wild-type genes (Burset and Guigo 1996; Burset et al,
2000, 2001). Its occurrence has not yet been described for plakophilin-1. Use of GC-cryptic splice sites in alternative splicing elicited by abolishment of the normal splice donor site is probably a rare event in human disease, as we have not been able to find similar instances in the literature. Recently, Hamada et al (2002) published a patient suffering from a mitigated form of McGrath syndrome, induced by a donor splice site mutation $(2021+1 G>A)$ causing in-frame cryptic splicing among other splicing products. However, while their patient presents complete hypotrichosis, our patient suffers only slight hair loss. The phenotype described here is therefore even milder.

In conclusion, we describe a patient suffering from ectodermal dysplasia-skin fragility syndrome caused by a novel splice site mutation. His phenotype may be ameliorated through partial protein rescue made possible by use of a non-consensus cryptic GC splice site, a phenomenon 


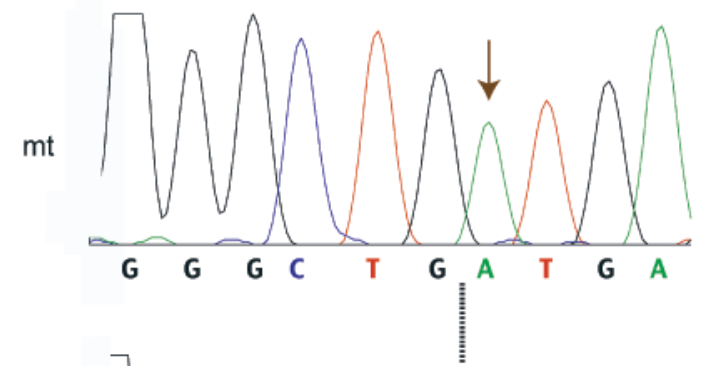

wt

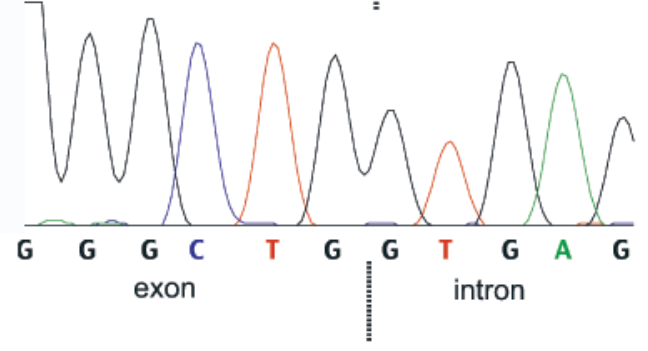

b

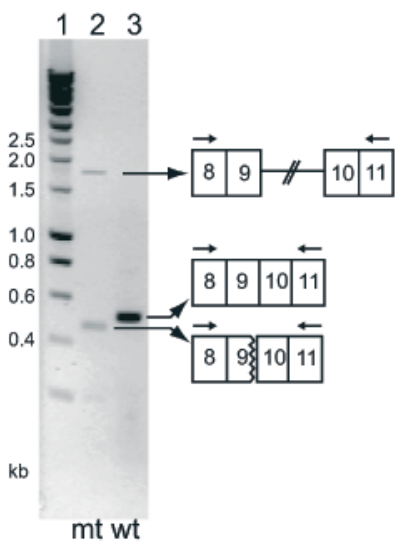

c

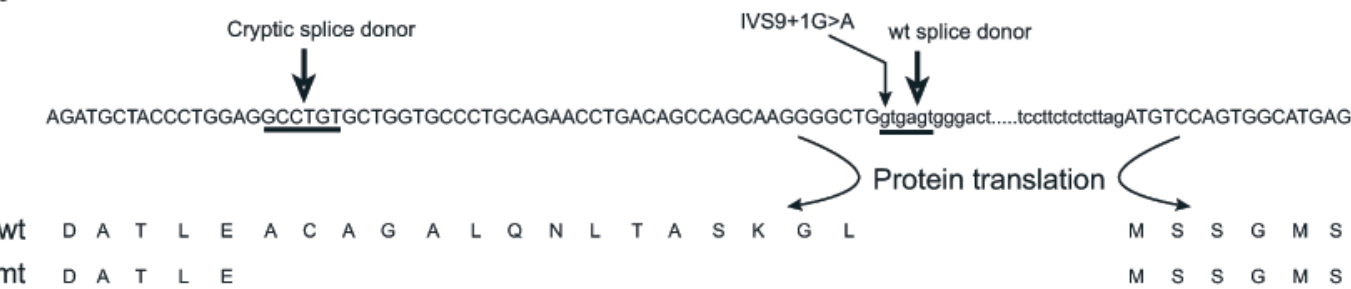

Figure 2

Mutation analysis and RT-PCR results. (a) Sequence traces showing the splice site mutation $(\mathrm{mt}=$ mutant, wt $=$ wild-type control). (b) Analysis of splicing products using RT-PCR with primers in PKP-1 exons 8 and 11 (8F: AGGTGCCCACCCGCTACC; 11R:AGCAGGCCGAGGACAAGATG) on patient skin RNA (mt, lane 2) and on control skin RNA (wt, lane 3). Splicing products are schematically depicted on the left with the upper DNA band patient skin RNA ( $\mathrm{mt}$, lane 2) and on control skin RNA (wt, lane 3). Splicing products are schematically depicted on the left with the upper DNA band
(lane 2) representing the splice product with intron 9 included (1759 bp) and the lower band (lane 2) representing the product on the right resulting (lane 2) representing the splice product with intron 9 included (1759 bp) and the lower band (lane 2) representing the product on the right resulting
from the cryptic splicing ( $477 \mathrm{bp}$ ). The wild-type product (lane 3) is $522 \mathrm{bp}$. Lane 1: molecular weight marker. PCR was performed according to the from the cryptic splicing ( $477 \mathrm{bp}$ ). The wild-type product (lane 3) is $522 \mathrm{bp}$. Lane 1: molecular weight marker. PCR was performed according to the
manufacturer's standard conditions (Invitrogen) with the initial step $90 \mathrm{~s}$ at $94^{\circ} \mathrm{C}$, then 35 cycles $30 \mathrm{~s}$ at $94^{\circ} \mathrm{C}, 30 \mathrm{~s}$ at $63^{\circ} \mathrm{C}, 60 \mathrm{~s}$ at $72^{\circ} \mathrm{C}$ and a final manufacturer's standard conditions (Invitrogen) with the initial step $90 \mathrm{~s}$ at $94^{\circ} \mathrm{C}$, then 35 cycles $30 \mathrm{~s}$ at $94^{\circ} \mathrm{C}, 30 \mathrm{~s}$ at $63^{\circ} \mathrm{C}, 60 \mathrm{~s}$ at $72^{\circ} \mathrm{C}$ and a final step of $420 \mathrm{~s}$ at $72^{\circ} \mathrm{C}$. (c) Schematic representation of the protein sequence resulting from RNA splicing using the cryptic GC site compared to the
wild-type sequence. The wild-type (wt) splice site (underlined) becomes non-functional by the splice site mutation (IVS9 + 1G $>A$ ) resulting in the use of a non-consensus GC cryptic splice site (underlined) within PKP-1 exon 9. After protein translation, the mt PKP-1 protein is effectively 15 amino acids internally shortened compared with the wt protein (lower panel).

that has not been previously described in the context of human disease. Our results point to unexpected facets of splicing regulation in the perspective of disease-causing mutations.

Peter M. Steijlen ${ }^{1 *}$, Maurice A. M. van Steensel ${ }^{1 *}$, Bastiaan J. H. Jansen ${ }^{1} \S$, Willeke Blokx ${ }^{2}$, Peter C. M. van de Kerkhof ${ }^{1}$, Rudolf Happle ${ }^{3}$,

${ }^{1}$ Department of Dermatology, University Hospital Nijmegen Netherlands: ${ }^{2}$ Department of Pathology, University Hospital Nijmegen,

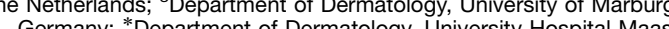
Germany; *Department of Dermatology, University Hospital Maastricht, Maastricht, the Netherlands; §Tumor Immunology, University Hospital Nijmegen, the Netherlands

M.A.M.S. is supported by grants from ZON-MW (NWO 920-03-085), Multigen BV, The Netherlands, a fellowship from the Berliner Stiftung für Dermatologie and a grant from "Stichting de Drie Lichten". B.J.H.J. and M.v.G. are supported by Multigen BV, The Netherlands.

DOI: 10.1111/j.0022-202X.2003.22302.x

Manuscript received January 24, 2003; revised August 17, 2003; accepted for publication September 4, 2003

Address correspondence to: M.A.M. van Steensel, Maastricht, PO Box 5800, 6202 AZ Maastricht, The Netherlands. Email: mvst@sder.azm.nl

\section{References}

Burset M, Guigo R: Evaluation of gene structure prediction programs. Genomics 34:353-367, 1996

Burset M, Seledtsov IA, Solovyev VV: Analysis of canonical and non-canonical splice sites in mammalian genomes. Nucleic Acids Res 28:4364-4375, 2000 


\section{LETTER TO THE EDITOR}

Burset M, Seledtsov IA, Solovyev WV: SpliceDB: Database of canonical and non-canonical mammalian splice sites. Nucleic Acids Res 29:255-259, 2001

Hamada T, South AP, Mitsuhashi Y, et al: Genotype-phenotype correlation in skin fragility-ectodermal dysplasia syndrome resulting from mutations in plakophilin 1. Exp Dermatol 11:107-114, 2002

Kowalczyk AP, Hatzfeld M, Bornslaeger EA, et al: The head domain of plakophilin-1 binds to desmoplakin and enhances its recruitment to desmosomes. Implications for cutaneous disease. J Biol Chem 274: 18145-18148, 1999

McGrath JA, Hoeger PH, Christiano AM, et al: Skin fragility and hypohidrotic ectodermal dysplasia resulting from ablation of plakophilin $1 . \mathrm{Br}$ Dermatol 140:297-307, 1999
THE JOURNAL OF INVESTIGATIVE DERMATOLOGY

McGrath JA, McMillan JR, Shemanko CS, et al: Mutations in the plakophilin 1 gene result in ectodermal dysplasia/skin fragility syndrome. Nat Genet
17:240-244, 1997

McMillan JR, Shimizu H: Desmosomes: Structure and function in normal and diseased epidermis. J Dermatol 28:291-298, 2001

Schmidt A, Langbein L, Rode M, Pratzel S, Zimbelmann R, Franke WW: Plakophilins 1a and 2b: Widespread nuclear proteins recruited in specific epithelial cells as desmosomal plaque components. Cell Tissue Res 290:481-499, 1997

Whittock NV, Haftek M, Angoulvant N, Wolf F, Perrot H, Eady RA, McGrath JA: Genomic amplification of the human plakophilin 1 gene and detection of a new mutation in ectodermal dysplasia/skin fragility syndrome. J Invest Dermatol 115:368-374, 2000 
Chapter 11 


\title{
A case of Rombo syndrome
}

\author{
M.A.M.VAN STEENSEL, N.G.J.JASPERS* AND P.M.STEIJLEN \\ Department of Dermatology, University Medical Centre St Radboud, PO Box 9101, 6500 HB Nijmegen, \\ The Netherlands \\ *Department of Cell Biology and Genetics, Erasmus University, Rotterdam, The Netherlands \\ Accepted for publication 17 January 2001
}

\begin{abstract}
Summary Rombo syndrome is a rare entity characterized by the presence of atrophoderma vermiculatum of the face, multiple milia, telangiectases, acral erythema and a propensity to develop basal cell carcinomas. We describe a patient whose clinical and histopathological abnormalities are consistent with this diagnosis.

Key words: Rombo syndrome
\end{abstract}

Rombo syndrome was first described in 1981 by Michaelsson et al. $;^{1}$ a second case report by Ashinoff et $a .^{2}$ describes an elderly patient supposedly suffering from it. It has some similarity to Bazex syndrome. ${ }^{3}$ Skin changes in Rombo syndrome first become evident at about the age of 7-10 years. At that time, a cyanotic redness as well as follicular atrophy of the sun-exposed skin becomes evident. Later, milia-like papules and telangiectases develop. The skin atrophy becomes more pronounced, leading to a 'worm-eaten' appearance of the skin known as atrophoderma vermiculatum. Histology of the skin shows an extremely aberrant distribution of elastin in the upper dermis, accompanied by vascular proliferation and a lymphocytic infiltrate.

We describe a patient suffering from a skin disorder that has the clinical and histological characteristics of Rombo syndrome. This is a sporadic patient; the original family had two instances of male to male transmission, suggesting that the phenotype may be transmitted as an autosomal dominant trait.

\section{Case report}

The patient, a 33-year-old man, first presented at age 15 years with complaints of red and irregular skin of the face. He also had multiple white papular lesions, especially on, but not limited to, the face. The skin changes had apparently started when he was about 6 years old, with redness and scaling, leading to considerable social impairment. After the initial

Correspondence: Maurice A.M.van Steensel.

E-mail: m.vansteensel@derma.azn.nl

(C) 2001 British Association of Dermatologists presentation, he was lost to follow-up. At his own request, he was again seen by us at age 33 years because of increasing redness and irregularity of the facial skin. He also complained of red and painful ears and redness of the skin of the lower arms. He did not use any medication. Family history was negative for skin disorders; his father had died of leukaemia at age 61 years and his mother of colon carcinoma at age 63 years. He had no allergies or other disorders.

Multiple whitish papules of a few millimetres in size were evident over the entire skin surface in a follicular distribution. Most were present on the face (Fig. 1a) and thorax. The facial skin over the cheeks was strikingly altered, seemingly indurated and erythematous with an irregular surface (Fig. 1b). The ears showed conspicuous telangiectases and erythema (Fig. 1c) and were very sensitive to even light touch.

Atrophoderma vermiculatum was present over both elbows. The skin of both lower arms and especially the hands was erythematous (Fig. 1d), becoming a normal colour upon lifting the arms. Scalp hair was long but thinly implanted. Eyebrows were thinly implanted especially on the lateral side, eyelashes were absent (Fig. 1e) and beard growth was very sparse. Axillary and pubic hair growth was normal. Nails and teeth did not show abnormalities. No mucosal abnormalities were found. The patient's build was peculiar, with a prominent mid-face, short stature and disproportion between the rump and legs (Fig. 1d).

Three punch biopsies were taken from the cysts on the patient's chest and a fourth was taken from indurated skin on the face. Biopsies taken from the chest lesions showed cystic structures located in the 

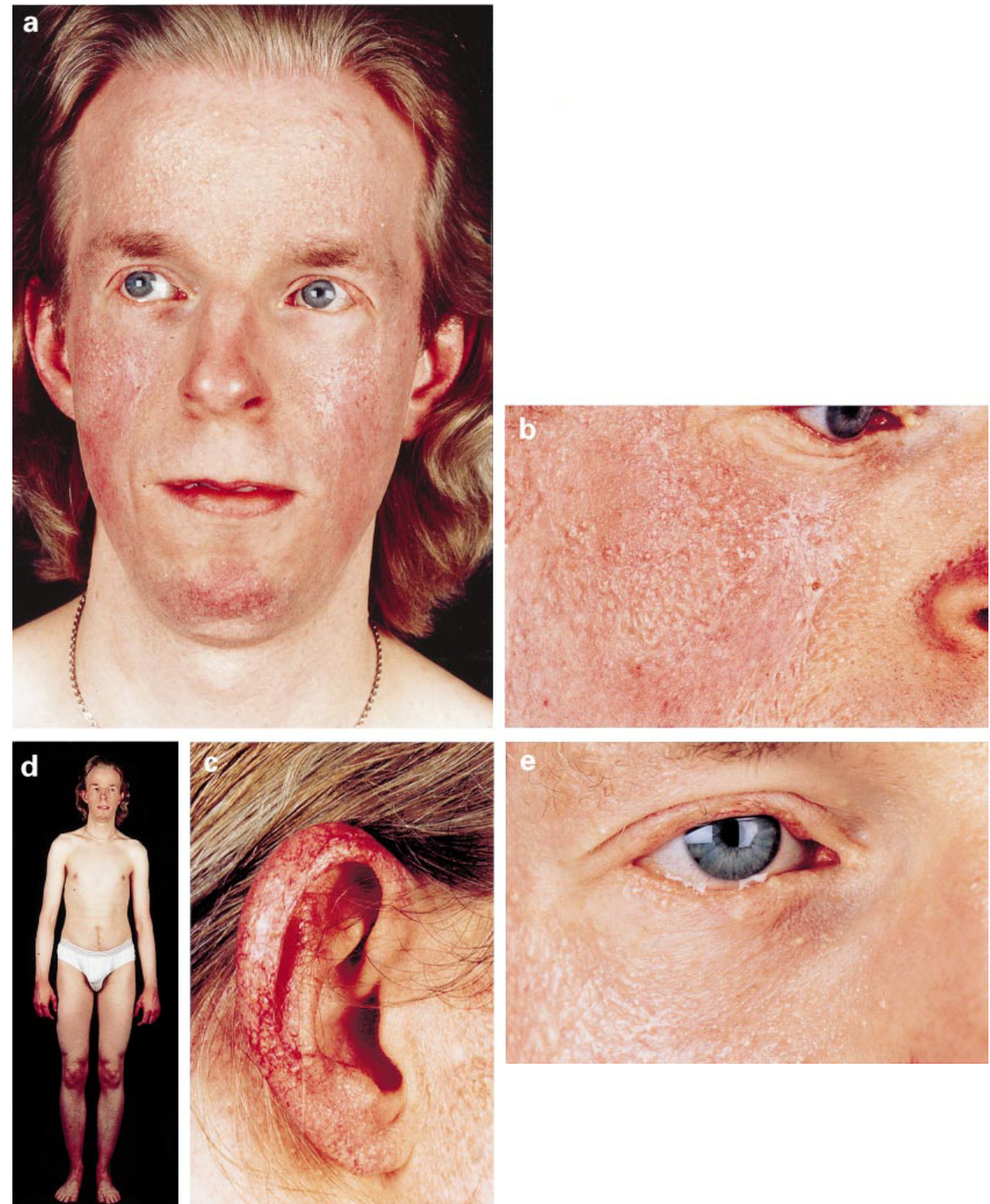

Figure 1. (a) Front view of patient. Note erythema of cheeks and chin, numerous milia, atrophoderma and lack of eyelashes. (b) Close-up of left cheek, showing atrophoderma vermiculatum and milia. (c) Close-up of left ear, showing erythema and telangiectaiases of the helix. (d) Front view of patient, total body, showing pronounced bluish erythema of the hands, an erythematous face and a peculiar build with a short trunk. (e) Closeup of left eye, showing milia, lack of eyelashes and thin implant of eyebrow. 

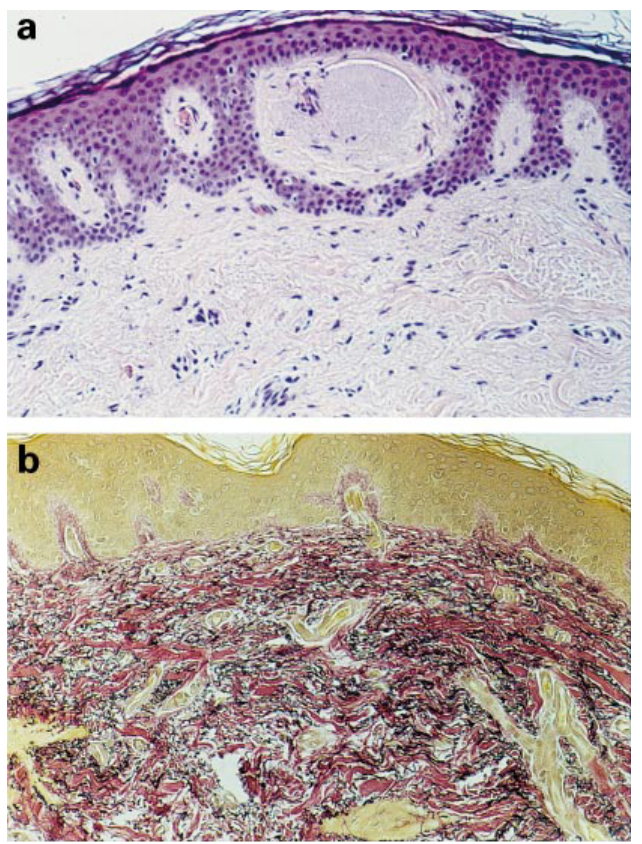

Figure 2. (a) Photomicrograph showing clumping of hyaline-like material directly below the epidermis and in the papillary dermis, a lymphocytic infiltrate around proliferating vessels, and hyalinization of collagen (haematoxylin and eosin; original magnification $\times 100$ ). (b) A highly irregular distribution of elastin is seen throughout the dermis, with clumping in some areas (van Giesson stain; original magnification $\times 40$ ).

middle dermis. These were covered on the inside with normal-appearing squamous epithelium. Inside the cysts, multiple vellus hairs and horny material were seen. The skin taken from the face showed moderate hyperorthokeratosis. High in the dermis, up to the papillary dermis, highly irregular deposits of wiry material were seen (Fig. 2a). A van Giesson stain showed that this was elastin. Clumps of elastin looking like swathes of steel wool were seen in one area, whereas other skin areas appeared devoid of elastin (Fig. 2b). The thickness of the bundles varied widely. Deeper down in the dermis, the collagen fibrils showed unusual changes consisting of hyalinization and vacuolization. Some proliferation of small vessels was noted, accompanied by a lymphocytic infiltrate.

\section{Discussion}

We describe a patient with skin abnormalities that are an almost exact copy of those described in the original patients with Rombo syndrome, detailed in the 1981 paper by Michaelsson et al. ${ }^{1}$ Major abnormalities leading to the diagnosis were the abnormal elastin distribution as seen in the biopsies, combined with multiple vellus hair cysts. The acral erythema and hypotrichosis provided a further clue to the diagnosis. The phenotype seems to be invariable, with abnormal elastin distribution in sun-exposed areas, acral erythema, vellus hair cysts or similar lesions, and hypotrichosis. Basal cell carcinomas may develop after the age of about 35 years, although the exact age at onset probably depends on exogenous factors such as exposure to sunlight. Eight patients are now known, including ours. Inheritance may be autosomal dominant, as two cases of male to male transmission have been recorded by Michaelsson et al. ${ }^{1}$ The patient described by Ashinoff et al. ${ }^{2}$ probably did not have Rombo syndrome. Skin abnormalities only became evident at an advanced age (the skin being normal at 35 years of age), acral erythema was lacking and the skin as seen on the photograph in the paper does not show atrophoderma vermiculatum. In addition, no milia-like lesions were present elsewhere on the body. Histology of affected skin was very different from that described by Michaelsson et al. ${ }^{1}$ and from that in our patient.

Histology of the affected skin in Rombo syndrome somewhat resembles that of solar elastosis, where thick aggregates of elastin are present in the upper dermis. ${ }^{1}$ The distribution is more regular in solar elastosis, however, and the elastin is separated from the epidermis by a band of normal collagen. This is in contrast to that seen in Rombo syndrome, where the distribution is highly irregular and the aggregates tend to be rounded rather than flattened as in solar elastosis. In addition, no separation of the elastin from the epidermis is evident.

Vellus hair cysts are not mentioned in the original paper, but the histology of the cysts described there is identical to that of the cysts we biopsied in our patient. According to Patrizi et al. the distinction between vellus hair cysts and other cystic lesions containing horny material is mostly semantic. ${ }^{4}$

The similarities to solar elastosis suggest that the Rombo syndrome gene may be involved in DNA repair and/or cell cycle regulation. The abnormal response of cultured fibroblasts to ultraviolet irradiation as described by Michaelsson et al. ${ }^{1}$ would be consistent with this hypothesis. The gene would have to be distinct from the ones presently known to be associated with DNA repair disorders, as the skin abnormalities seen in these are very different from those seen in Rombo syndrome. The presence of vellus hair cysts may 
M.A.M.VAN STEENSEL $e t$ al.

provide a clue: the gene is apparently involved in hair follicle growth and differentiation.

Mutations in the Hairless gene, which is involved in the regulation of apoptosis during hair follicle cycling, ${ }^{5}$ cause the recessive disorder atrichia with papular lesions. The latter are cystic hair follicle remnants sometimes filled with hair material. The resemblance suggests that vellus hair cysts may have a similar pathogenetic mechanism. In that case, the Rombo syndrome gene may be involved in the regulation of apoptosis. Programmed cell death is important for the response to DNA damage and it is possible that the Rombo syndrome defect disturbs this response. We are currently investigating this hypothesis.

\section{Acknowledgments}

M.A.M.van Steensel is supported by grants from the
Netherlands Organization for Scientific Research (NWO grant no. 920-03-085) and Rebirth SA.

\section{References}

1 Michaelsson G, Olsson E, Westermark P. The Rombo syndrome: familial disorder with vermiculate atrophoderma, milia, hypotrichosis, trichoepitheliomas, basal cell carcinomas and peripheral vasodilation with cyanosis. Acta Derm Venereol (Stockh) 1981; 61: 497-503.

2 Ashinoff R, Jacobson M, Belsito DV. Rombo syndrome: a second case report and review. J Am Acad Dermatol 1993; 28: 1011-14.

3 Hauck RM, Manders EK. Familial syndromes with skin tumor markers. Ann Plast Surg 1994: 33: 102-11.

4 Patrizi A, Neri I, Guerrini V et al. Persistent milia, steatocystoma multiplex and eruptive vellus hair cysts: variable expression of multiple pilosebaceous cysts within an affected family. Dermatology 1998; 196: 392-6.

5 Ahmad W, Ul HMF, Brancolini $V$ et al. Alopecia universalis associated with a mutation in the human hairless gene. Science 1998; 279: 720-4. 
Chapter 12 


\title{
Woolly hair, premature loss of teeth, nail dystrophy, acral hyperkeratosis and facial abnormalities: possible new syndrome in a Dutch kindred
}

\author{
M.A.M.VAN STEENSEL, M.I.KOEDAM,* O.Q.SWINKELS, F.RIETVELD $\dagger$ AND \\ P.M.STEIJLEN \\ Departments of Dermatology and †Pathology, University Medical Center Nijmegen, PO Box 9101, 6500 HB Nijmegen, \\ The Netherlands \\ *Isala Clinics, Zwolle, The Netherlands \\ Accepted for publication 25 January 2001
}

Summary We describe a Dutch kindred with a possibly novel dominant syndrome of premature loss of curly, brittle hair, premature loss of teeth due to caries, nail dystrophy and acral keratoderma. We discuss the possibility that this ectodermal dysplasia of group 1-2-3-4 is a variant of known disorders such as pachyonychia congenita. We conclude that none of these diagnoses fits the symptoms we observe in our patients and propose the name curly hair-acral keratoderma-caries syndrome in view of the most obvious abnormalities.

Key words: ectodermal dysplasia, hair, keratoderma, nails, syndrome, teeth

\begin{abstract}
Abnormalities of hair and teeth are seen in many congenital disorders. Little is known about the regulation of the growth and differentiation of ectodermal appendages, although recent hair research ${ }^{1}$ has started to shed some light on these complex processes. Syndromes in which multiple ectodermal appendages are affected are of particular importance for the study of skin biology, as the association of symptoms indicates that the development of the affected structures has a common molecular denominator.

The identification of large kindreds suffering from ectodermal dysplasias is important, because in such families linkage analysis may be used to identify the causative gene. Here, we report a Dutch family in which multiple members are affected by an apparently novel syndrome.
\end{abstract}

\section{Case reports}

The proposita (III-5 in the pedigree) first visited the dermatology outpatient clinic of the Isala Hospital

Correspondence: Maurice A.M.van Steensel.

E-mail: m.vansteensel@derma.azn.nl
(Zwolle, the Netherlands) at 35 years of age with unruly, brittle hair and premature hair loss. Other complaints included hypohidrosis, partial loss of teeth due to caries necessitating a dental prosthesis at age 15 years and thickening of the nails of the hands and feet. Her general health was good. According to the patient, other members of her family had similar problems. Physical examination showed a receding frontal hairline, curly, dry and brittle hair and sparse eyebrows and eyelashes. The upper teeth were missing and had been replaced by a dental prosthesis. The nails of the fingers and toes were yellow and thickened. The malar region appeared slightly flattened and frontal bossing was noted (Fig. 1a,b). The patient was referred to the Department of Dermatology, University Medical Center, Nijmegen, the Netherlands for further evaluation. Apart from the abnormalities described above, we noted a keratoderma with a reticulate dark pattern on the tips of the fingers and toes (Fig. 2). Findings in her eldest brother (III-1) were more pronounced. He also had a flat malar region and frontal bossing (Fig. 3a,b). Another brother (III-4) was most severely affected, with complete loss of teeth and baldness. We next visited the entire family and identified multiple affected persons (Fig. 4) of different ages, the youngest being 9 years of 

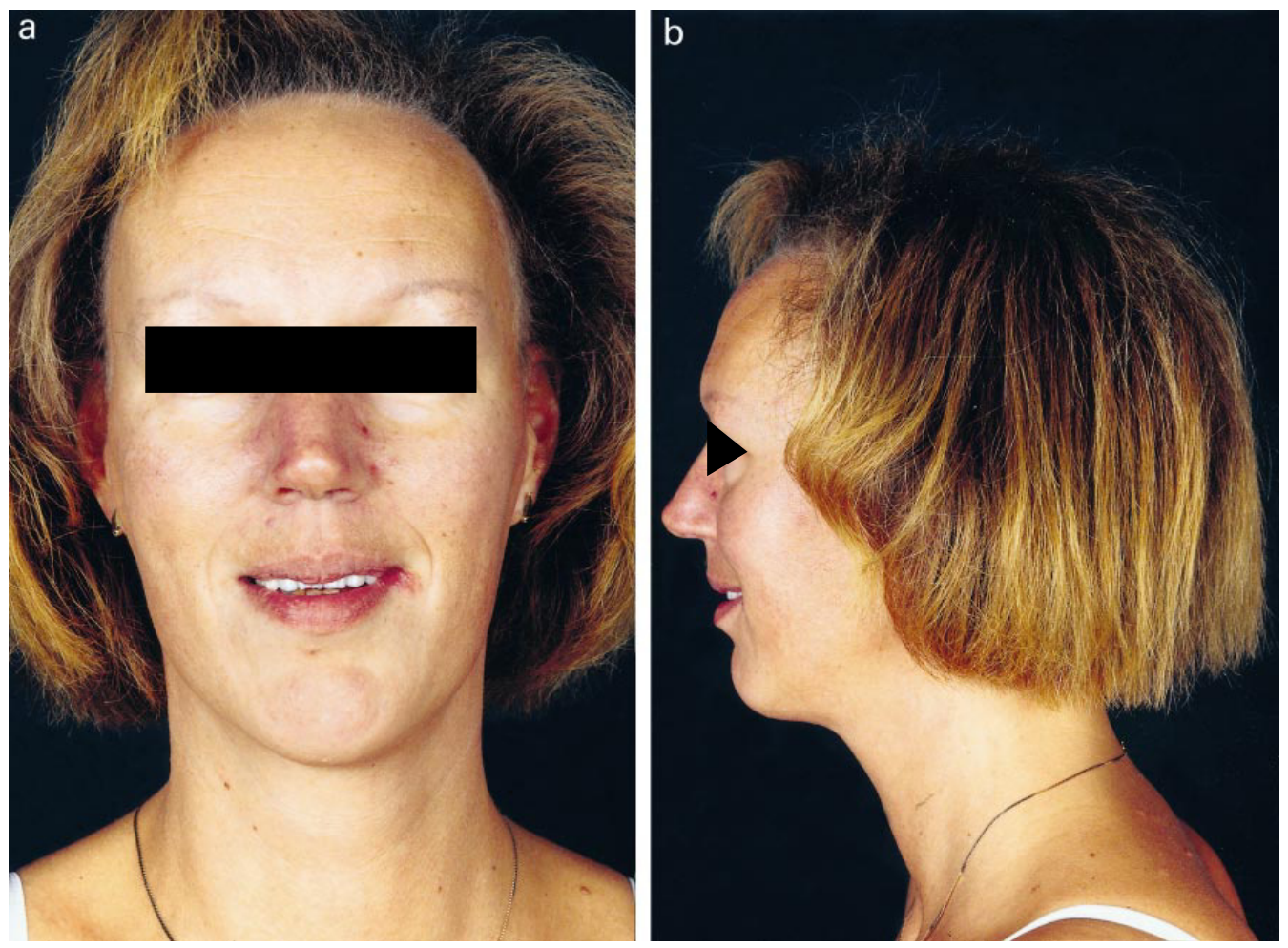

Figure 1. (a) The index patient, frontal view. Note high frontal hairline, dry hair and lack of eyelashes on lower and most of upper eyelids. Eyebrows are pencil stripes. (b) The index patient, lateral view. Flat malar region and prominent forehead. Dry hair, especially near the tips.

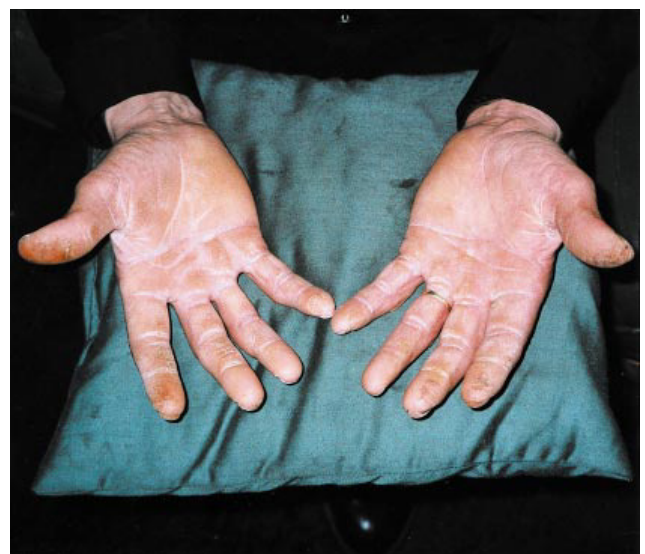

Figure 2. The hands of the eldest brother of the index patient show keratoderma with accentuated palmar creases and dark hyperkeratosis, especially on the tips of the thumb and index finger. age. Features in all affected individuals were identical, although severity appeared to increase with age. The flattening of the malar region and the frontal bossing were variable, not every affected family member showing these abnormalities to the same degree. Affected family members were photographed and blood samples were taken from the entire family for future molecular studies.

\section{Additional investigations}

Scanning electron microscopy (SEM) of hairs taken from the proposita and her elder brother revealed several abnormalities. The appearance of the hair shafts was documented using a modified version of the scoring system proposed by Micali et al. ${ }^{2}$ (see Table 1). The most prominent abnormalities seen on SEM were a marked variation in diameter and multiple torsions of hair shafts. Many hairs showed longitudinal 

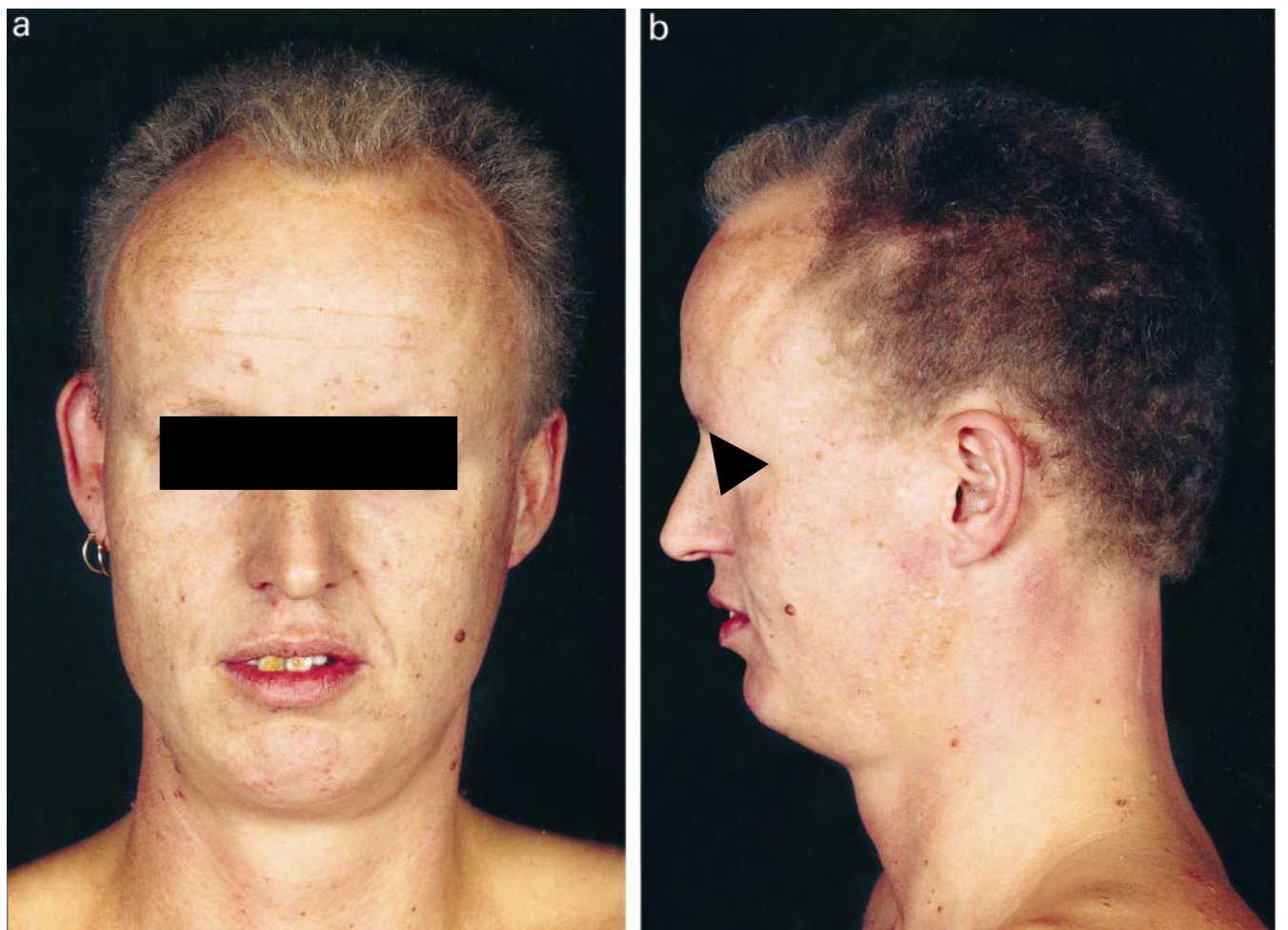

Figure 3. (a) The index patient's eldest brother, frontal view. Dry, sparse hair with receding frontal hairline and Geheimratsecken. Lack of eyelashes and eyebrows, and pronounced caries of incisors. (b) Same patient, lateral view. Flat malar region and prominent forehead. Sparse hair in temporoparietal region.

grooves and an absent cuticle. Cross-sections were oval to triangular. Some hairs showed unusual torsions with grooves, which appeared to run across the torsions.

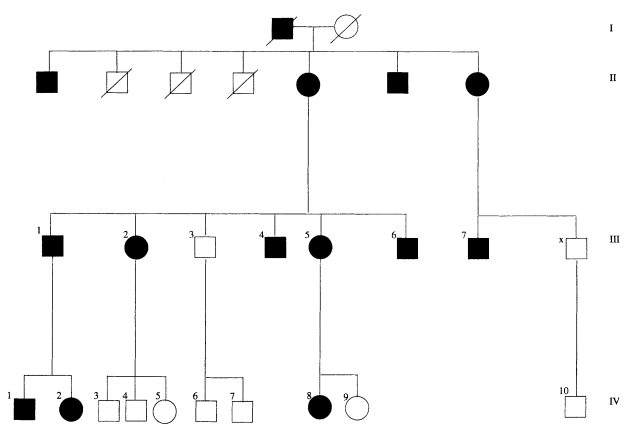

\section{Discussion}

Many ectodermal dysplasias are characterized by hair, tooth and nail abnormalities. The London Dysmorphology Database ${ }^{3}$ lists 16 syndromes with the combination of kinky/curly OR coarse hair and caries OR enamel abnormalities (Boolean search terms are in upper case). It is not clear whether the caries is a primary event or secondary to enamel hypoplasia. The scarce literature on this point seems to suggest the latter. $^{4}$

Of the 16 syndromes, the pili torti-enamel hypoplaFigure 4. Pedigree of the family. sia syndrome most resembles the syndrome we describe

(c) 2001 British Association of Dermatologists, British Journal of Dermatology, 145, 157-161 
M.A.M.VAN STEENSEL et al.

Table 1. Hair shaft abnormalities scored on scanning electron microscopy

\begin{tabular}{lc}
\hline Abnormality & Presence or absence \\
\hline Distorted bulb & - \\
Bifid bulb & - \\
Small bulb & - \\
Transverse bulb markings & - \\
Follicular wall ridging & - \\
Follicular wall damage & - \\
Abnormal non-smooth & + \\
cuticular layer & + \\
Smooth cuticular layer & + \\
Absent cuticle & $($ patchy) \\
(patchy/complete) & - \\
Transverse groove marking & - \\
Follicular damage & - \\
Longitudinal ridging & + \\
Bent shaft & + \\
Longitudinal groove (no. of & $(1)$ \\
grooves on circumference) & +++ \\
Torsion & +++ \\
Variation in diameter (range) & $(25-100 \mu \mathrm{m})$ \\
Shape on cross-section & Oval to triangular \\
\hline Adapted from Micali et al. &
\end{tabular}

here. Freire-Maia and Pinheiro described this entity. ${ }^{5}$ Features are keratosis pilaris, dry fair hair, enamel hypoplasia and widely spaced, abnormal teeth. The keratosis pilaris as well as the wide spacing of abnormal teeth distinguish it from the syndrome we describe. The hair abnormalities we observe are not consistent with a diagnosis of pili torti. Instead, they are more like those seen in other ectodermal dysplasias. Twisting of the hair shafts in pili torti is much more pronounced and grooves are not present. The cuticle is generally intact. ${ }^{6,7}$ There is no mention in the literature of the reticulate acral keratoderma seen in our patients.

Clouston syndrome was also considered in the differential diagnosis. However, the teeth are normal in this condition, ${ }^{8}$ and there is no hypohidrosis. This sets this condition apart from the disorder we describe here. Pachyonychia congenita (PC) can be associated with dry hair, hypotrichosis and abnormal teeth and resembles the condition we describe. However, the Online Mendelian Inheritance in Man database ${ }^{9}$ does not list caries as one of the symptoms of PC. The caries, nail dystrophy and acral keratoderma in our patients differ from what is seen in PC. Woolly hair and the SEM abnormalities seen in our patients have, to our knowledge, not been reported in PC. Two disorders characterized by curly hair are the tricho-dento-osseous syndrome and the curly hairankyloblepharon-nail dysplasia syndrome. ${ }^{10-12}$ The first is distinguished by changes in skull bone density, not by mid-face hypoplasia. The second is a recessive condition characterized by ankyloblepharon at birth. All of these features are lacking in our patients and the curling of the hair seen in those patients is different from that seen in ours. Finally, the flattening of the malar region and the frontal bossing distinguish the condition we describe from all other syndromes mentioned above and may be the most important defining features. Table 2 summarizes the similarities and differences between these syndromes and the disease we describe here.

In conclusion, the familial occurrence of a unique combination of ectodermal abnormalities and facial

Table 2. Features of syndromes in the differential diagnosis

\begin{tabular}{|c|c|c|c|c|c|c|}
\hline & Caries & Hypohidrosis & $\begin{array}{c}\text { Acral } \\
\text { keratoderma }\end{array}$ & $\begin{array}{c}\text { Woolly } \\
\text { hair }\end{array}$ & $\begin{array}{c}\text { Nail } \\
\text { dystrophy }\end{array}$ & $\begin{array}{c}\text { Flat } \\
\text { mid-face }\end{array}$ \\
\hline CHACS & + & + & + & + & $\stackrel{+}{\text { (discoloration) }}$ & + \\
\hline $\mathrm{PC}$ & + & - & \pm & - & $\begin{array}{c}+ \\
\text { (thickening) }\end{array}$ & - \\
\hline $\begin{array}{l}\text { Clouston } \\
\text { syndrome }\end{array}$ & - & - & - & - & (brittle) $^{+}$ & - \\
\hline TDO & + & - & - & $\begin{array}{c} \pm \\
\text { (curly) }\end{array}$ & - & - \\
\hline CHANDS & - & - & - & $\begin{array}{c} \pm \\
\text { (curly) }\end{array}$ & - & - \\
\hline $\begin{array}{l}\text { Pili torti-enamel } \\
\text { hypoplasia }\end{array}$ & + & - & - & + & - & - \\
\hline
\end{tabular}


dysmorphism probably represents a novel syndrome for which we propose the name curly hair-acral keratoderma-caries syndrome.

\section{Acknowledgments}

We thank the members of the family for their cooperation. M.v.S. is supported by Netherlands Organization for Research grant NWO 920-03-085 and a grant from Rebirth SA.

\section{References}

1 van Steensel MAM, Steijlen PM, Happle R. Molecular genetics of the hair follicle-the state of the art. Proc Soc Exp Biol Med 2000; 223: $1-7$.

2 Micali G, Cook B, Blekys I, Solomon LM. Structural hair abnormalities in ectodermal dysplasia. Pediatr Dermatol 1990; 7 $27-32$.

3 Winter RM, Baraitser M. The London Dysmorphology Database. J Med Genet 1987; 24: 509-10.

4 Kantaputra PN, Pruksachatkunakorn C, Vanittanakom P. Rapp-Hodgkin syndrome with palmoplantar keratoderma, glossy tongue, congenital absence of lingual frenum and of sublingual caruncles: newly recognized findings. Am J Med Genet 1998; 79 $343-6$.

5 Freire-Maia N, Pinheiro M. Ectodermal Dysplasias: a Clinical and Genetic Study. New York: Alan R.Liss Inc., 1984: 142-4.

6 Maruyama T, Toyoda M, Kanei A, Morohashi M. Pathogenesis in pili torti: morphological study. J Dermatol Sci 1994; 7 (Suppl.): S5-12.

7 Dawber RPR, Comaish S. Scanning electron microscopy of normal and abnormal hair shafts. Arch Dermatol 1970; 101: 316-22.

8 Hassed SJ, Kincannon JM, Arnold GL. Clouston syndrome: an ectodermal dysplasia without significant dental findings. $\mathrm{Am}$ Med Genet 1996; 61: 274-6.

9 Online Mendelian Inheritance in Man OMIM $^{\mathrm{TM}}$. McKusickNathans Institute for Genetic Medicine, Johns Hopkins University (Baltimore, MD) and National Center for Biotechnology Information, National Library of Medicine (Bethesda, MD), 2000. World Wide Web URL: http://www.ncbi.nlm.nih.gov/omim/

10 Wright JT, Kula K, Hall K et al. Analysis of the tricho-dentoosseous syndrome genotype and phenotype. Am J Med Genet 1997; 72: 197-204

11 Baughman FA Jr. CHANDS: the curly hair-ankyloblepharonnail dysplasia syndrome. Birth Defects Orig Artic Ser 1971; 7: $100-2$.

12 Toriello HV, Lindstrom JA, Waterman DF, Baughman FA Re-evaluation of CHANDS. J Med Genet 1979; 16: 316-17. 
Chapter 13 


\title{
CASE REPORT
}

\section{New syndrome of hypotrichosis, striate palmoplantar keratoderma, acro-osteolysis and periodontitis not due to mutations in cathepsin $\mathrm{C}$}

\author{
M.A.M.VAN STEENSEL, M.VAN GEEL AND P.M.STEIJLEN \\ Department of Dermatology, University Medical Centre St Radboud Nijmegen, PO Box 9101, 6500 HB Nijmegen, the Netherlands \\ Accepted for publication 7 February 2002
}

Summary We report a mother and daughter with a syndrome of hypotrichosis, striate palmoplantar keratoderma, onychogryphosis, periodontitis, acro-osteolysis and psoriasis-like skin lesions. The syndrome resembles Papillon-Lefèvre syndrome (PLS), characterized by palmoplantar keratoderma, periodontitis and psoriasis-like skin lesions, and particularly Haim-Munk syndrome, an allelic variant of PLS with acro-osteolysis. Both are caused by mutations in the cathepsin C gene (CTSC). Our patients differ in the unique nature of the palmar keratoderma and hypotrichosis. We have sequenced CTSC in the mother without finding mutations in either coding or non-coding parts of the gene. We propose that our patients suffer from a new syndrome possibly caused by mutations in a gene that has a functional or structural relation with CTSC.

Key words: acro-osteolysis, cathepsin C, Haim-Munk syndrome, hyperkeratosis, onychogryphosis, periodontitis

Papillon-Lefèvre syndrome (PLS; MIM 245000) is characterized by pronounced palmoplantar hyperkeratosis, psoriasis-like lesions on the extremities and periodontitis leading to early loss of teeth. It was recently shown to be due to mutations in the cathepsin $\mathrm{C}$ gene (CTSC). ${ }^{1,2}$ In all cases described so far, inheritance was autosomal recessive. A rare allelic variant is called Haim-Munk syndrome (MIM 245010). ${ }^{3}$ It is distinguished from PLS by the additional symptoms of acro-osteolysis, arachnodactyly and pes planus. The patients with Haim-Munk syndrome described in the literature were all related to a single family of Jews who emigrated from Cochin, India, to Israel. We report a woman of Dutch descent suffering from a syndrome that resembles Haim-Munk syndrome. Inheritance may be autosomal dominant as her daughter, born after artificial insemination, has identical symptoms. Our patients have additional symptoms including hypotrichosis totalis. We show that the syndrome is not caused by mutations in CTSC and thus constitutes a

Correspondence: M.A.M.van Steensel.

E-mail: m.vansteensel@derma.azn.nl new entity. We propose the name 'HOPP syndrome'. This is an acronym for hypotrichosis-osteolysis-periodontitis-palmoplantar keratoderma syndrome, after the defining symptoms.

\section{Case report}

A 52-year-old woman of Dutch ancestry was referred to our out-patient clinic for evaluation of her life-long ectodermal dysplasia. She gave a history of dystrophic nails and absent eyebrows and lashes since birth, with thickened palmoplantar skin since the age of 2 years. At age 7 years, her scalp hair, which had always been thinly implanted, started to fall out. The hair loss was not accompanied by other symptoms such as pustules, itching or scaling. Cutting her strongly curved nails was nearly impossible, as it was very painful and accompanied by bleeding. Around the same time, her teeth began to be affected by caries and periodontitis became apparent. At about 15 years of age, erythematous scaly lesions appeared on the lower arms and legs. When the patient was 18 years old, the skin of the little fingers of both hands and of the index finger of the 
right hand was treated by $\mathrm{X}$-irradiation, resulting in a decrease of the hyperkeratosis. Three years later, all the teeth were extracted because of advanced caries and a dental prosthesis was fitted. A few years after that, all nails were surgically removed, followed by radical nail bed excisions and free skin grafting to cover the resulting defects. The patient recently developed a ventricular tachycardia of unknown origin, which is being treated with a beta-adrenergic receptor blocker and acenocoumarol. Electron microscopic examination of the hair was performed at another centre and reportedly showed pili torti et annulati. No diagnosis was made at that time, but the patient was told that the disorder was autosomal recessive with a $25 \%$ chance of recurrence should she have children with her husband, a second-degree cousin (Fig. 1).

On examination, we noted a peculiar, reticulate pitted hyperkeratosis of the palms, spiky hyperkeratosis of the soles, hypotrichosis universalis (Figs 2-4) and periodontitis with absence of all teeth. We also noted a lingua plicata. In addition, all nails were missing, many fingers showing the scars of surgical nail removal and subsequent skin grafting. Photographs of both hands taken 25 years ago showed obvious onychogryphosis (Fig. 5). Several fingers apparently lacked distal pha-

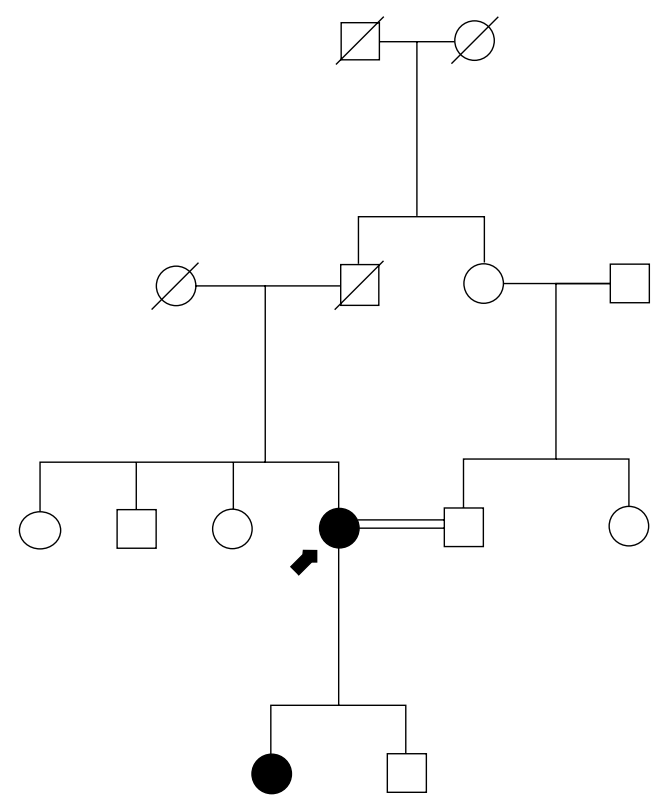

Figure 1. Pedigree of the family.
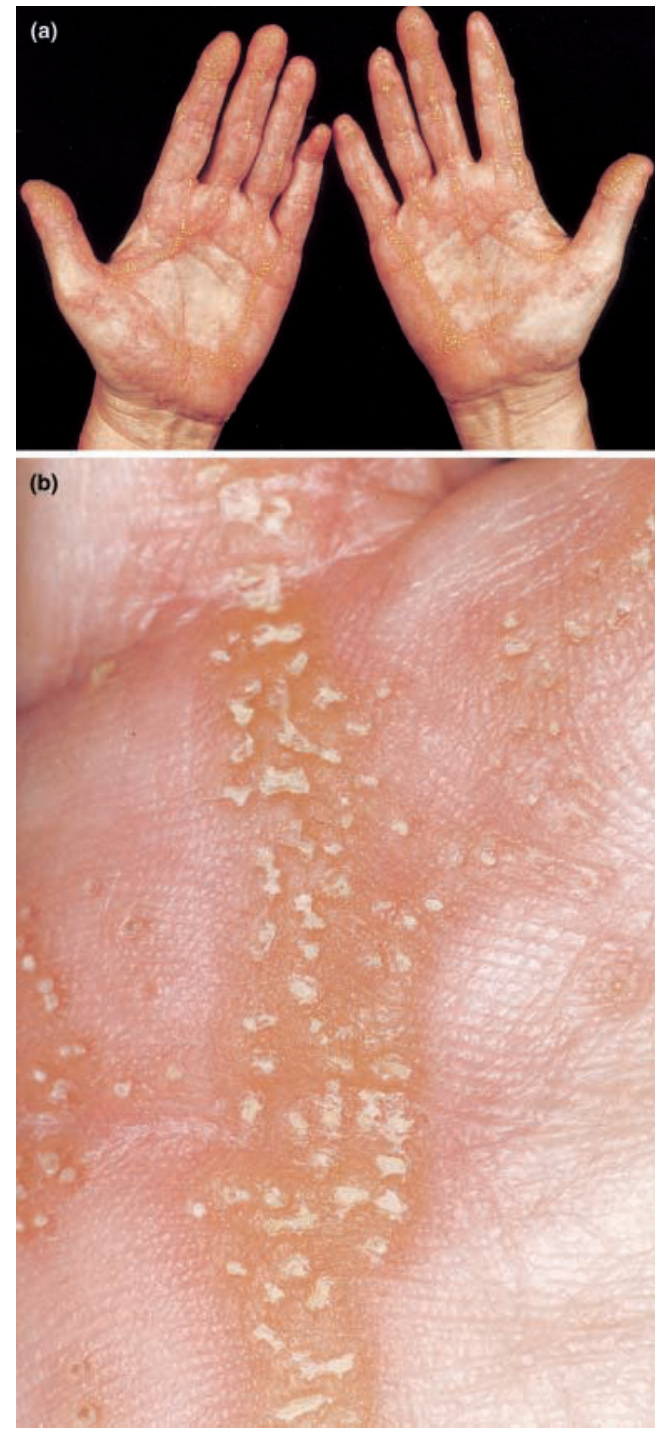

Figure 2. (a) Hyperkeratosis of the palms. Notice the peculiar reticular pattern and reduction defects of the fingertips. (b) Close-up of the palmar hyperkeratosis showing 'pitting'.

langes. All digits were thin and tapered towards the tips. Erythematous scaling lesions resembling psoriatic plaques were visible on the lower arms and upper legs. Some hairs remained on the occipital and temporal regions of the scalp. The hypotrichosis of the scalp 


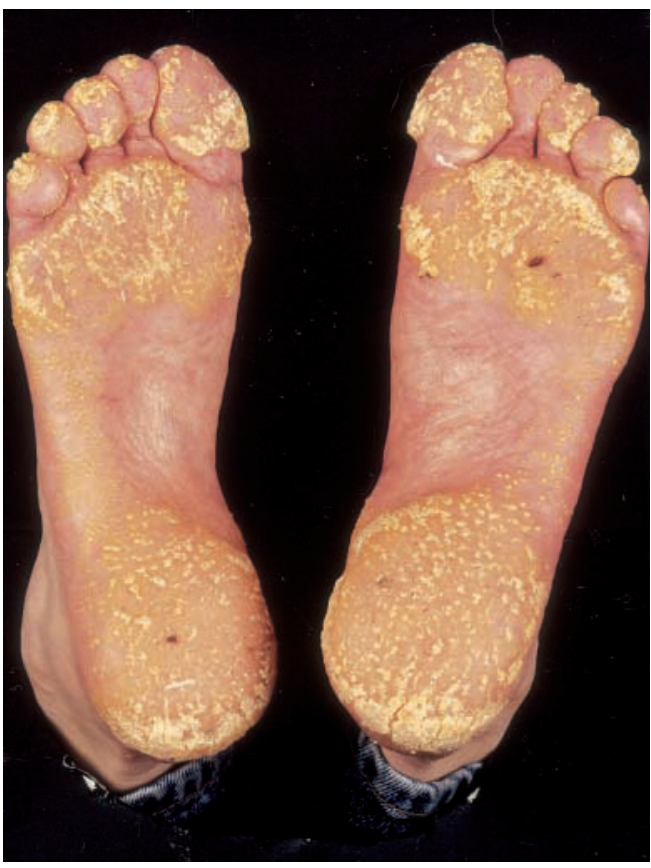

Figure 3. Yellow spiky hyperkeratosis is evident on the soles.

seemed secondary to scarring alopecia, as hair follicle openings were missing over most of the scalp. The hyperkeratosis of the palms was highly unusual, following a reticular pattern and showing many small 'pinprick' pits.

The patient has two children, a 15-year-old daughter and a 17-year-old son, both born after artificial insemination, as the patient and her husband wished to prevent transmission of her supposedly autosomal recessive disorder. The son is healthy, but the daughter is affected by what appears to be the same disorder as her mother. In her case, symptoms first appeared at the age of 3 months and consisted of unspecified nail abnormalities. At the age of 2 years, hyperkeratoses of the feet appeared. At the age of 13 years, she started suffering from joint pains. These were diagnosed as 'severe psoriatic arthritis' and methotrexate treatment was prescribed. We were able to examine the daughter and revise the X-ray photographs. The latter showed no joint abnormalities. Periarticular bone density was decreased, suggesting that the daughter, too, might be suffering from osteolysis. Physical examination showed onychogryphosis (Fig. 6a), hypotrichosis, periodontitis

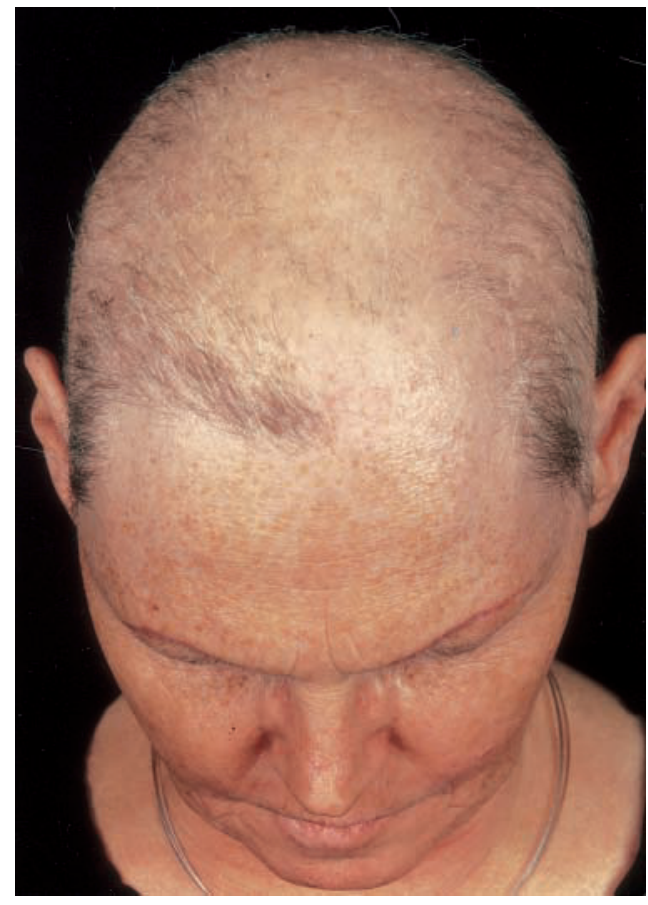

Figure 4. Hypotrichosis affects most of the scalp. The eyebrows are pencil stripes.

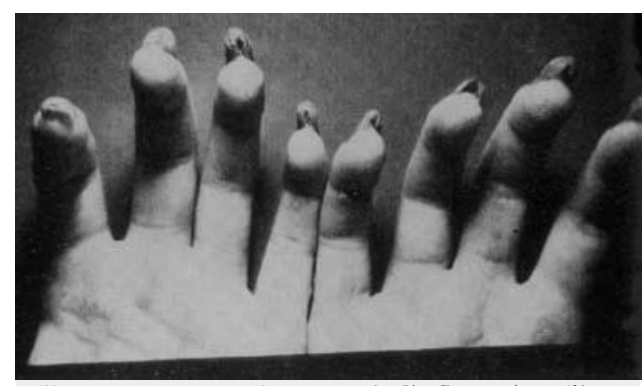

Fig. 2. Polykeratosis congenitalis Touraine (lineaire hyperkeratose der handpalmen en (subunguale hyperkeratose).

Figure 5. A 25-year-old photograph of the patient's hands showing pronounced onychogryphosis and linear keratoderma. A translation of the caption is 'Polykeratosis congenitalis Touraine (linear hyperkeratosis of the palms and subungual hyperkeratosis)'.

and lingua plicata (Fig. 6b). She also had palmoplantar keratoderma, but in her case the palmar keratoderma was nummular rather than linear (Fig. 6a). The

(C) 2002 British Association of Dermatologists, British Journal of Dermatology, 147, 575-581 


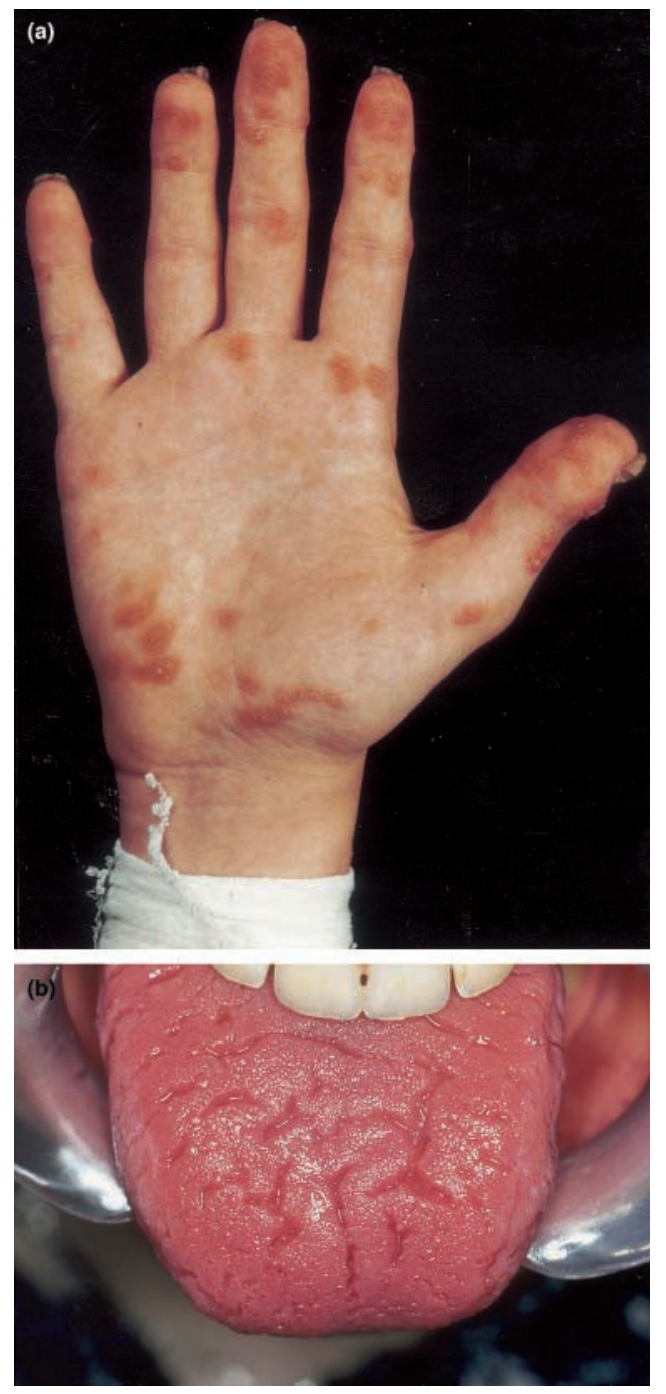

Figure 6. (a) Daughter's right hand, palmar view. Notice nummular hyperkeratosis and onychogryphosis. (b) Lingua plicata in the daughter.

plantar keratoderma was similar to that seen in her mother. She had erythematous, scaling lesions on the lower arms and legs. The hypotrichosis and periodontitis were less severe than in the mother. The skin and joint symptoms reportedly responded well to the methotrexate treatment. There were no other affected family members and the patient's parents (both deceased from unrelated causes) were not related.

We performed several additional investigations on the mother to establish a diagnosis; the daughter refused any such procedure. A punch biopsy was taken from a hyperkeratotic area on the right hand and a psoriasis-like lesion on the lower right leg, and a wedge biopsy from the scalp in an area where some hairs were present. We also took X-rays of the hands. We isolated DNA from whole blood and analysed CTSC for mutations using genomic polymerase chain reaction. Histology of the punch biopsy from the hyperkeratotic area showed abnormalities consistent with a diagnosis of hyperkeratosis. No cornoid lamellae were visible; parakeratosis could not be demonstrated. The affected skin showed pronounced

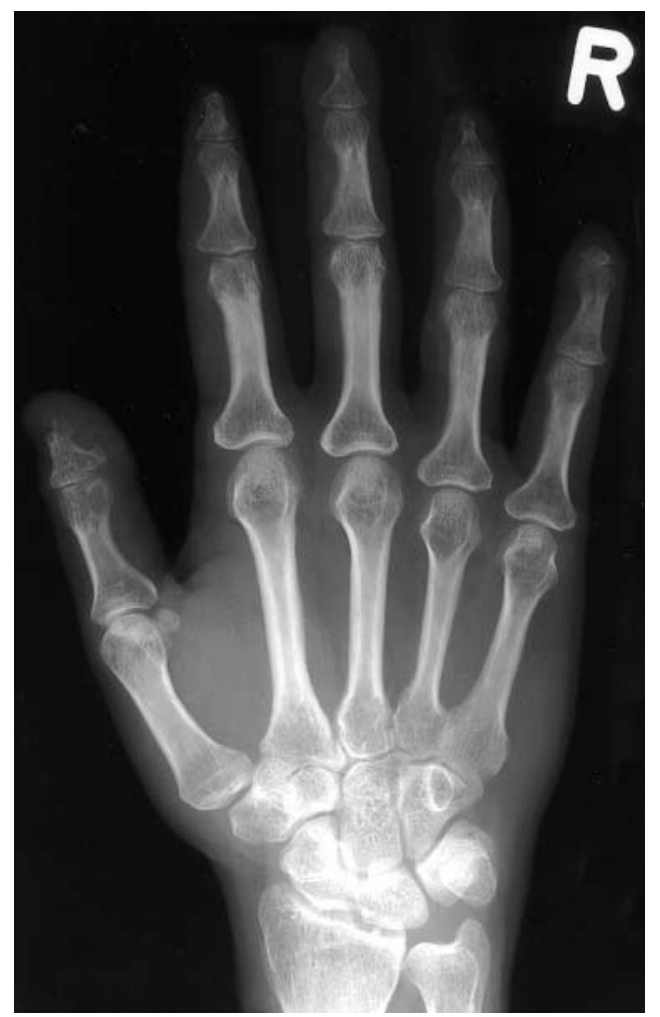

Figure 7. X-ray photograph of the patient's right hand. Note decreased epimetaphyseal bone density of phalanges, missing terminal phalanx of right little finger, and claw-like tufting of remaining distal phalanges. 
orthohyperkeratosis but other abnormalities were not seen. The biopsy from the leg showed hyperplasia and hyperparakeratosis. The granular layer was absent. The dermal papillae were elongated but did not show the tongue shape typical for psoriasis. In the upper dermis, there was a perivascular lymphocytic infiltrate. A biopsy slide of the daughter was obtained from another hospital and showed identical abnormalities. Examination of the scalp biopsy showed a reduced number of hair follicles and only slight scarring. A mild lymphocytic infiltrate was seen surrounding some hair follicles. X-rays showed acroosteolysis of all digits, most pronounced in the little finger of the right hand. Here, the distal phalanx was totally absent (Fig. 7). The remaining distal phalanges showed claw-like tufts as described previously by Puliyel and Sridharan Iyer. ${ }^{4}$ Lastly, we sequenced all seven coding exons, including intron-exon junctions, of CTSC using intronic primers as listed in Table 1. We found no pathogenic mutations in the exons or in splice acceptor and donor sites. We also sequenced about 150 nucleotides of the $5^{\prime}$ and $3^{\prime}$ untranslated

Table 1. Polymerase chain reaction (PCR) primer sequences

\begin{tabular}{ll}
\hline Exon & \multicolumn{1}{c}{ Primer sequence } \\
\hline Exon 1 forward & 5'-CAATCCCCTGCTGCTCAGTG-3' \\
Exon 1 reverse & 5'-AAGCGGTAGTTGGCGTGGC-3' \\
Exon 2 forward & 5'-GACTGTGCTCAAACTGGGTAG-3' \\
Exon 2 reverse & 5'-CTACTAATCAGAAGAGGTTTCAG-3' \\
Exon 3 forward & 5'-GGGGCACATTTACTGTGAATG-3' \\
Exon 3 reverse & 5'-CGTATGTCTCATTTGTAGCAAC-3' \\
Exon 4 forward & 5'-GTACCACTTTCCACTTAGGCA-3' \\
Exon 4 reverse & 5'-GGAGGATGGTATTCAGCATTC-3' \\
Exon 5 forward & 5'-CCTAGCTAGTCTGGTAGCTG-3' \\
Exon 5 reverse & 5'-GTATCCCCGAAATCCATCACA-3' \\
Exon 6 forward & 5'-CTCTGTGAGGCTTCAGATGTC-3' \\
Exon 6 reverse & 5'-CAACAGCCAGCTGCACACAG-3' \\
Exon 7a forward & 5'-TTGTGGGCTATGGCACTGACTC-3' \\
Exon 7a reverse & 5'-GCTTCTGAGATTGCTGCTGAAAG-3' \\
Exon 7b forward & 5'-CTTTCAGCAGCAATCTCAGAAGC-3' \\
Exon 7b reverse & 5'-TCTCAGACTCATCAAACATCCAAGG-3' \\
Exon 7a forward & 5'-GGGGTAACCATGTGTGTTATTCA-3' \\
Exon 7b reverse & 5'-CCCCTTTACAACTGATGCAGA-3' \\
\hline
\end{tabular}

PCR conditions were as follows: $5 \mathrm{~min}$ initial denaturation at $95^{\circ} \mathrm{C}$ followed by 35 cycles of $95^{\circ} \mathrm{C}$ for $1 \mathrm{~min}, 55^{\circ} \mathrm{C}$ for $30 \mathrm{~s}, 72^{\circ} \mathrm{C}$ for $30 \mathrm{~s}$. For primer pairs 1 and $7 \mathrm{a}$ the annealing temperature was $50{ }^{\circ} \mathrm{C}$. A final extension step of $72{ }^{\circ} \mathrm{C}$ for $5 \mathrm{~min}$ was included. Prior to the sequencing reactions, the PCR products were purified using the shrimp alkaline phosphatase/exonuclease presequencing kit (USB Science). Sequencing was performed using the BigDyeDeoxy Terminator kit (Applied Biosystems) and sequencing reactions were analysed on an ABI 3700 capillary sequencer. Sequence fragments were assembled and analysed for mutations using either the Phred-PhrapConsed ${ }^{11-13}$ or ContigXpress (Informax, Inc.) contig assembly software packages.

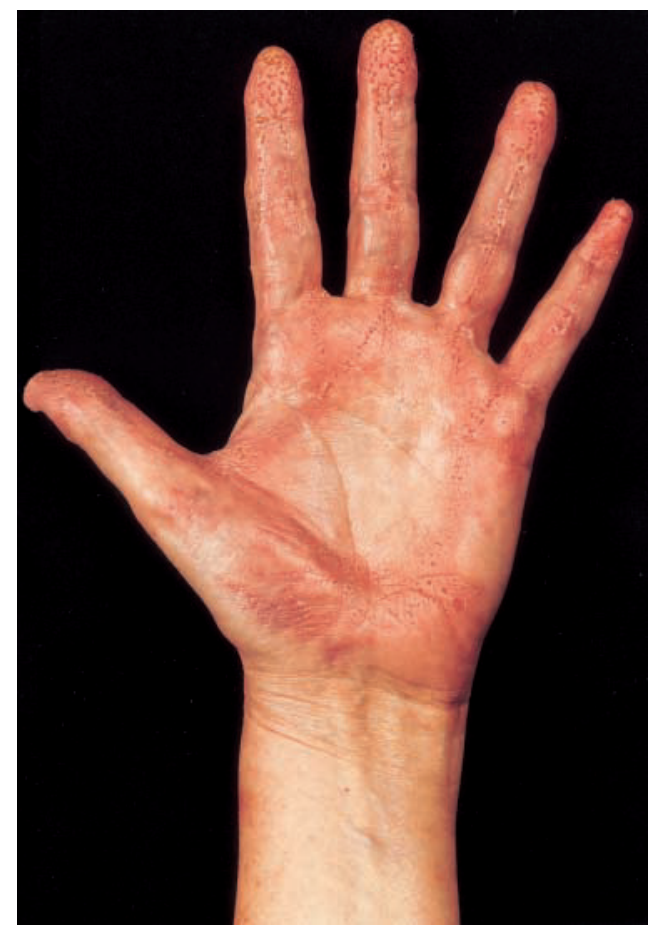

Figure 8. The patient's left hand, palmar view, after 6 weeks on acitretin $35 \mathrm{mg}$ daily. Notice black 'spikes' in linear distribution. The yellow hyperkeratosis has disappeared.

regions without finding changes from the published sequence. We did detect a heterozygous C824 $\rightarrow \mathrm{T}$ polymorphism that changes an ATC codon to ATT in exon 6. Both code for threonine.

As the patient considered her palmoplantar keratoderma to be the most disabling of her complaints, we treated her with acitretin $35 \mathrm{mg}$ daily. After 6 weeks of treatment the palmoplantar keratoderma had regressed considerably, leaving clearly visible black pits in place of the yellow keratoses (Fig. 8). The side-effects were well tolerated and the patient was satisfied with the result.

\section{Discussion}

The combination of onychogryphosis, acro-osteolysis, palmoplantar keratoderma and periodontitis has been previously described by other authors and is presently known as Haim-Munk syndrome. We sequenced CTSC and found no mutations. This suggests that 
M.A.M.VAN STEENSEL et al.

Table 2. Symptom matrix

\begin{tabular}{|c|c|c|c|c|c|c|c|c|c|}
\hline Syndrome & $\begin{array}{l}\text { Periodon- } \\
\text { titis }\end{array}$ & $\begin{array}{l}\text { Lingua } \\
\text { plicata }\end{array}$ & $\begin{array}{l}\text { Psoriasiform } \\
\text { lesions }\end{array}$ & $\begin{array}{l}\text { Palmoplantar } \\
\text { keratoderma }\end{array}$ & $\begin{array}{l}\text { Onycho- } \\
\text { gryphosis }\end{array}$ & $\begin{array}{c}\text { Acro- } \\
\text { osteolysis }\end{array}$ & $\begin{array}{c}\text { Pes } \\
\text { planus }\end{array}$ & Hypotrichosis & $\begin{array}{l}\text { Ventricular } \\
\text { arrhythmias }\end{array}$ \\
\hline Papillon-Lefèvre & + & - & + & + & - & - & - & - & - \\
\hline Haim-Munk & + & - & + & ++ & + & + & + & - & - \\
\hline Naxos disease & - & - & - & + & - & - & - & - & + \\
\hline $\begin{array}{l}\text { Desmoplakin } \\
\text { disease }\end{array}$ & - & - & - & + & - & - & - & - & + \\
\hline Our cases & + & + & + & ++ & + & + & - & + & + \\
\hline
\end{tabular}

our patient suffers from a previously undescribed disorder that shares many symptoms with HaimMunk syndrome but is a distinct entity. This notion is supported by the finding of additional symptoms that have not been previously described in Haim-Munk syndrome patients: hypotrichosis, linear/reticular palmar keratoderma, lingua plicata and ventricular arrhythmias. Another symptom of Haim-Munk syndrome, pes planus, is lacking in our patient. Her daughter has an identical phenotype, indicating that the association with hypotrichosis is probably not spurious.

The mode of inheritance is uncertain. We had no opportunity to test for paternity and therefore cannot rule out recessive inheritance. X-linked dominant inheritance cannot be ruled out, although the lack of a mosaic distribution of the lesions argues against this mode of inheritance. If we accept that the daughter is indeed the product of artificial insemination, autosomal dominant inheritance is the most likely in this case. Our mutation analysis showed no mutations in the coding sequence and splice donor/acceptor sites of CTSC, suggesting that the disorder we describe here is a new syndrome. This notion is supported by the obvious differences with Haim-Munk syndrome, PLS and other syndromes, as summarized in Table 2.

One of the striking aspects of the phenotype was the linear and reticulate hyperkeratosis of both hands. Superficially, it resembled a porokeratotic eccrine and ostial duct naevus. Histology, however, showed orthohyperkeratosis not limited to the eccrine ducts. The linear pattern on the fingers somewhat resembles the hyperkeratosis seen in keratoderma palmaris et plantaris striata et areata Siemen-Wachter. This disorder is characterized by linear and nummular hyperkeratoses of the hands and feet. In one family the disorder was shown to be caused by mutations in the adhesion molecule desmoglein $1 .^{5}$ Thus, the causative gene may have a functional or structural relationship with desmoglein 1.
Hypotrichosis is not a part of either PLS or HaimMunk syndrome. Lingua plicata, or 'scrotal' tongue, has been described as an isolated entity, but also in the context of inherited disorders. ${ }^{6,7}$ Both hypotrichosis and lingua plicata are rare traits and may therefore be part of the syndrome. The pedigree is too small to draw a firm conclusion in this regard. The same caveat applies to the ventricular tachycardia of our patient. In Naxos disease, a disorder caused by mutations in the plakoglobin gene, ventricular tachycardias and other cardiac disorders are combined with palmoplantar keratoderma. ${ }^{8}$ A related disorder has recently been described and is caused by desmoplakin mutations. ${ }^{9}$ However, in both disorders the hair is woolly and hypotrichosis is not a feature.

Acro-osteolysis is one of the most striking aspects of the phenotype and is a prominent feature of HaimMunk syndrome. This symptom is also seen in pycnodysostosis and Hajdu-Cheney syndrome. The former disorder is caused by mutations in the cathepsin $\mathrm{K}$ gene that is expressed in osteoclast lysosomes. ${ }^{10}$ Cathepsin $\mathrm{C}$ probably functions in osteoclasts as well, considering the acro-osteolysis in Haim-Munk syndrome. Because acro-osteolysis is a rare symptom, it is likely to be highly specific for a disturbance in osteoclast function related to defective functioning of lysosomal endopeptidases such as cathepsins $\mathrm{K}$ and $\mathrm{C}$. It is possible that the gene causing our patient's syndrome has a function that is very similar to that of cathepsins $\mathrm{C}$ and $\mathrm{K}$ in osteoclast lysosomes and skin.

In conclusion, we describe a patient and her daughter, both suffering from a novel, possibly autosomal dominant syndrome resembling Haim-Munk syndrome. We have excluded CTSC as the cause by means of direct mutation analysis, confirming that the syndrome we describe is distinct from Haim-Munk syndrome but is perhaps caused by a defect in a gene that has a structural or functional relation with CTSC. We suggest the designation 'HOPP syndrome' as an acronym for the distinguishing symptoms. 


\section{Acknowledgments}

M.A.M.van Steensel is supported by grants from NWO (Dutch Organization for Scientific Research) no. 92005-083, Rebirth SA, Luxembourg, Multigen Inc., the Netherlands and the Berliner Stiftung für Dermatologie, Germany. M.van Geel is supported by grants from Rebirth SA, Luxembourg and Multigen Inc., the Netherlands. The authors thank the expert reviewer for his help in improving the paper.

\section{References}

1 Toomes C, James J, Wood AJ et al. Loss-of-function mutations in the cathepsin $\mathrm{C}$ gene result in periodontal disease and palmoplantar keratosis. Nat Genet 1999; 23: 421-4.

2 Hart TC, Hart PS, Bowden DW et al. Mutations of the cathepsin C gene are responsible for Papillon-Lefèvre syndrome. J Med Genet 1999; 36: 881-7.

3 Hart TC, Hart PS, Michalec MD et al. Haim-Munk syndrome and Papillon-Lefèvre syndrome are allelic mutations in cathepsin C. J Med Genet 2000; 37: 88-94.

4 Puliyel JM. Sridharan Iyer KS. A syndrome of keratosis palmoplantaris congenita, pes planus, onychogryphosis, periodontosis, arachnodactyly and a peculiar acro-osteolysis. $\mathrm{Br}$ J Dermatol 1986; 115: 243-8.
5 Rickman L, Simrak D, Stevens HP et al. N-terminal deletion in a desmosomal cadherin causes the autosomal dominant skin disease striate palmoplantar keratoderma. Hum Mol Genet 1999; 8: 971-6.

6 Kullaa-Mikkonen A. Familial study of fissured tongue. Scand J Dent Res 1988; 96: 366-75.

7 Levenson MJ, Ingerman M, Grimes C et al. Melkersson-Rosenthal syndrome. Arch Otolaryngol 1984; 110: 540-2.

8 McKoy G, Protonotarios N, Crosby A et al. Identification of a deletion in plakoglobin in arrhythmogenic right ventricular cardiomyopathy with palmoplantar keratoderma and woolly hair (Naxos disease). Lancet 2000; 355: 2119-24.

9 Norgett EE, Hatsell SJ, Carvajal-Huerta L et al. Recessive mutation in desmoplakin disrupts desmoplakin-intermediate filament interactions and causes dilated cardiomyopathy, woolly hair and keratoderma. Hum Mol Genet 2000; 9: 2761-6.

10 Gelb BD, Shi GP, Chapman HA et al. Pycnodysostosis, a lysosomal disease caused by cathepsin K deficiency. Science 1996; 273: 1236-8.

11 Gordon D, Abajian C, Green P. Consed: a graphical tool for sequence finishing. Genome Res 1998; 8: 195-202.

12 Ewing B, Green P. Base-calling of automated sequencer traces using phred. II. Error probabilities. Genome Res 1998; 8: 186-94.

13 Ewing B, Hillier L, Wendl MC et al. Base-calling of automated sequencer traces using phred. I. Accuracy assessment. Genome Res 1998; 8: 175-85. 
Chapter 14 


\section{A third case of HOPP syndrome-confirmation of the phenotype}

DOI: $10.1111 / \mathrm{j} .1365-2133.2004 .05939 . \mathrm{x}$

SIR, In the September 2002 issue of the British Journal of Dermatology, we described a possibly novel syndrome of hypotrichosis, acro-osteolysis, palmoplantar keratoderma, peridontitis and onychogryphosis that we named 'HOPP' syndrome after the most obvious abnormalities. ${ }^{1}$ We reported on two affected patients, a mother and daughter. The palmar keratoderma followed a highly unusual reticular pattern. In addition, both patients had a lingua plicata, which symptom we did not at that time consider to be part of the phenotype. The syndrome has been recognized in the OMIM database (entry 607658) but until now has remained to be confirmed as a separate entity by reports of unrelated patients.
Here, we report on a third, unrelated patient from Venezuela suffering from what appears to be HOPP syndrome. His phenotype is almost identical to that of the two Dutch patients. This report confirms the existence of HOPP syndrome as a unique entity and further delineates the phenotype.

A 24-year-old male presented to the Department of Dermatology with palmoplantar keratoderma, onychogryphosis, psoriasiform plaques and alopecia. As an infant he had been evaluated for pili annulati and curved nails. Later, punctate palmoplantar keratoderma with keratotic papules on the knees and elbows became evident. At 4 years of age he started to lose his hair and psoriasiform plaques appeared on his trunk. There was no family history of a similar disorder; the patient's parents were not consanguineous. After the initial presentation, the patient was lost to follow-up.

On examination he was completely bald, with only a few eyelashes remaining (Fig. 1a). Examination of the scalp
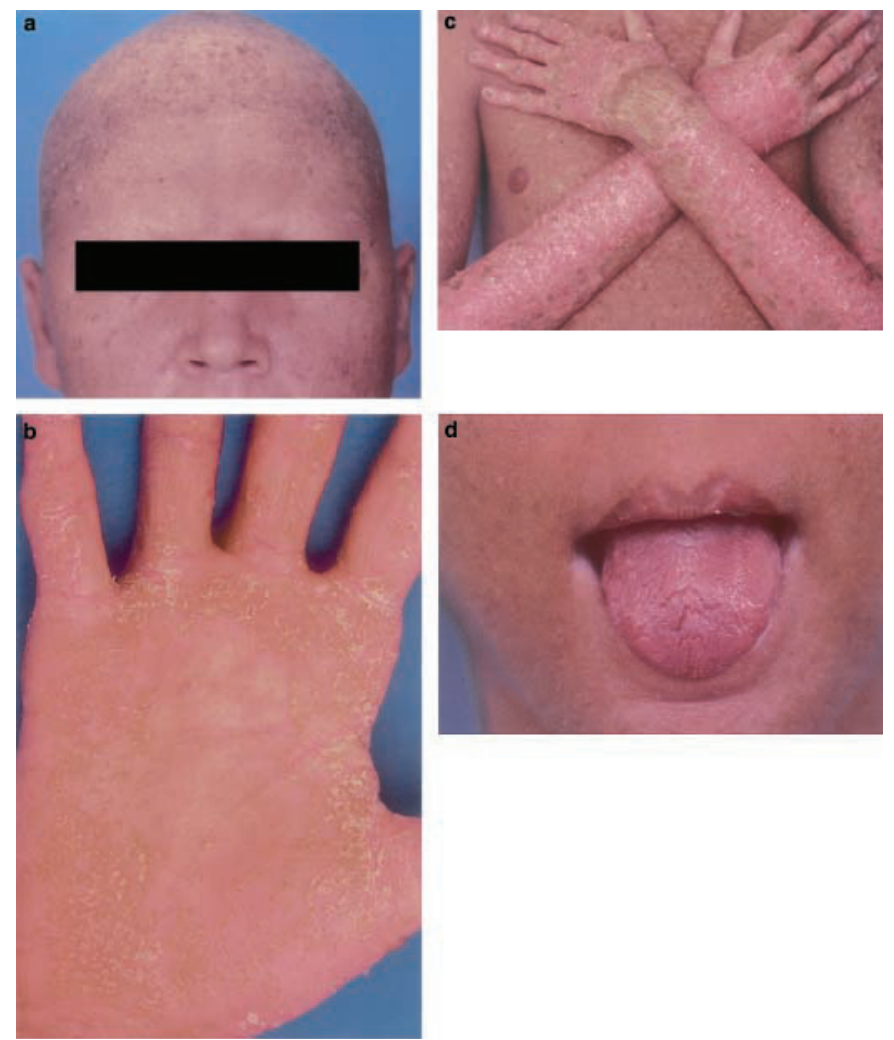

Figure 1. On examination the patient was completely bald, with only a few eyelashes remaining (a). The palmoplantar keratoderma followed a reticulate pattern, with multiple punctate, pitted keratoses on the hands and feet (b). On his head, trunk and limbs there were dyschromic overlapping round and oval macules, with colours ranging from off-white to brown to almost black (c). Examination of the oral cavity showed lingua plicata and gingivitis (d). 
suggested that the alopecia might be the result of a scarring process. The palmoplantar keratoderma followed a reticulate pattern, with multiple punctate, pitted keratoses on the hands and feet (Fig. 1b). There was extensive onychogryphosis, with long, thin fingers. On his head, trunk and limbs there were dyschromic overlapping round and oval macules, with colours ranging from off-white to brown to almost black (Fig. 1c). These lesions were not associated with atrophy or telangiectasias. On his extremities there were extensive psoriasiform plaques. Examination of the oral cavity showed lingua plicata and gingivitis (Fig. 1d). A biopsy of his palm showed a nonepidermolytic hyperkeratosis. A biopsy of the dyschromia on his back showed a hyperpigmented basal layer with foci of absence of melanin. No inflammatory infiltrate was observed. A biopsy of the scalp could not be performed. $\mathrm{X}$-rays of the fingers did not show clear-cut acro-osteolysis.

We report on a young male from Venezuela suffering from what appears to be HOPP syndrome (OMIM 607658), although acro-osteolysis of the fingers could not be unequivocally demonstrated by X-ray examination. However, the other symptoms exactly match those first described by us in 2002. ${ }^{1}$ Obviously, Papillon-Lefèvre syndrome should be considered in the differential diagnosis, but the hypotrichosis and peculiar palmoplantar keratoderma suffice to distinguish the syndrome in this patient. This report confirms the existence of HOPP syndrome as a defined entity and further delineates the phenotype. It is of interest to note that the lingua plicata, which was considered a possible minor accompanying malformation in our first two patients, is also seen in this new case. Furrowing and grooving of the tongue does occur in $5 \%$ of the normal population (Gorlin, personal communication to McKusick, 1982), but the prior probability of finding this variant in three people suffering from a rare disorder seems very low. Hence, lingua plicata is probably part of the phenotype. That acro-osteolysis seems to be absent may indicate that it is a variable part of the phenotype. However, in the original patients, it was not present to the same degree in mother and daughter either. It may well be a symptom that slowly develops with age, in which case the present patient will yet develop it. Pili annulati, seen in one of the original patients, was also seen in this case. The occurrence in at least two of three HOPP syndrome patients suggests that it may be part of the phenotype. It does occur as an isolated or autosomal dominant anomaly but is also seen in the context of other syndromes such as pilodental dysplasia with refractive errors. ${ }^{2}$ In HOPP syndrome, it may reflect the dysfunction of the hair follicle that eventually results in its loss. Co-segregation of the two traits seems unlikely because it would have to have occurred twice in unrelated patients; the prior chance of observing this is low. When in doubt about the diagnosis, for instance when hypotrichosis is not (yet) present, pili annulati may serve to differentiate HOPP syndrome from related phenotypes.

Of particular interest in this patient is the dyschromia. It is not a poikiloderma, as there are no telangiectasias. A mixture of postinflammatory hyper- and hypopigmentation should be considered given the history of widespread psoriasiform skin lesions that may very well have left their mark. However, an intriguing possibility is that the dyschromia is an idiopathic one. It resembles dyschromia hereditaria symmetrica of Dohi, a congenital disorder that has been linked to two loci on 1q21 and $6 \mathrm{q} 24 .^{3,4}$ The intriguing possibility of a contiguous gene syndrome thus presents itself. No obvious candidate genes for HOPP syndrome could be located using the human genome browser at http://genome.ucsc.edu but the idea seems worth pursuing. Alternatively, reticulate pigmentation of DowlingDegos or a Civatte poikiloderma might be considered, although hypopigmentation is not a feature of either disorder. At present, it is unclear whether the dyschromia is part of the phenotype.

In conclusion, we describe here a third case of HOPP syndrome. The symptoms in this patient are almost identical, although he does not seem to suffer from acro-osteolysis. Hence, the latter may be a variable part of the phenotype. The phenotype is remarkably constant across ethnic boundaries, suggesting limited genetic background influence and a crucial role for the causative gene in normal skin function.

Servicio de Dermatología, Hospital Ruiz y Páez, Ciudad Bolívar, Venezuela, and ${ }^{*}$ Dermatology

Department, University Hospital

Maastricht, P.O. Box 5800, 6202

AZ Maastricht, the Netherlands.

Correspondence: M.A.M.van Steensel,

E-mail: mvst@sder.azm.nl

\section{References}

1 Van Steensel MA, Van Geel M, Steijlen PM. New syndrome of hypotrichosis, striate palmoplantar keratoderma, acro-osteolysis and periodontitis not due to mutations in cathepsin C. Br J Dermatol 2002; 147: 575-81.

2 Kopysc Z, Barczyk K, Krol E. A new syndrome in the group of euhidrotic ectodermal dysplasia. Pilodental dysplasia with refractive errors. Hum Genet 1985; 70: 376-8.

3 Xing $\mathrm{OH}$, Wang MT, Chen XD et al. A gene locus responsible for dyschromatosis symmetrica hereditaria (DSH) maps to chromosome 6q24.2-q25.2. Am J Hum Genet 2003; 73: 377-82.

4 Zhang XJ, Gao M, Li M et al. Identification of a locus for dyschromatosis symmetrica hereditaria at chromosome 1q11-1q21. J Invest Dermatol 2003; 120: 776-80. 
Chapter 15 
Conrad GLADE Mauria A.M. van STEENSEL Peter M. STEIJLEN

\section{Hypotrichosis, lymphedema of the legs and acral telangiectasias - new syndrome?}

We describe a girl of Turkish descent suffering from a peculiar combination of symptoms. The presenting complaint was bilateral lymphedema of the legs; additional symptoms include hypotrichosis, telangiectasias and angiomata limited to acral regions. We discuss the possibility that this girl suffers from Noonan/cardio-facio-cutaneous syndrome. We conclude that the combination of symptoms listed here probably represents a new syndrome for which we propose the name hypotrichosis-lymphedema-telangiectasia syndrome. (Key words: hypotrichosis, lymphedema, telangiectasia, syndrome.)

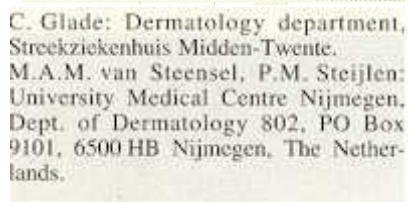

Reprints: M.A.M. van Steensel.

Fax: (+31) 243541184

e-mail: m.vansteensel@derma.azn.nl
L ymphedema of the legs combined with hair abnormalities can be a prominent feature of Noonan syndrome (MIM 163950). This phenotype has previously been referred to as "male Turner syndrome" because of the similarities with Turner $(45, \mathrm{X} 0)$ syndrome. The most prominent characteristics are short stature, webbed neck, triangular facies and sparse, sometimes woolly or fragile hair. Congenital heart disorders can also be a part of the phenotype as can the skin disorder ulerythema ophryogenes. If the latter anomalies dominate the phenotype, it is called cardio-facio-cutaneous syndrome (CFC, MIM 115150). Noonan and CFC syndrome are obviously allelic [1].

Here we describe a girl of Turkish descent suffering from a combination of symptoms that bears some resemblance to Noonan/CFC syndrome but seems sufficiently distinct to warrant description as a separate entity.

\section{Case report}

\section{History}

The patient, a 12 year-old female, is the first child of consanguineous (first cousins) Turkish parents (Fig. 1). A younger sib is healthy. At the age of four years, swelling of the lower legs appeared. Initially, only the left leg was affected. Exercise apparently aggravated the swelling. Later, reddish papules and maculae developed on the hands and feet. The scalp hair had always been thin and did not grow well. Eyebrows had always been sparse and eyelashes had never been present.

The swelling of the legs was treated with compressive stockings, reportedly with satisfactory results. There were no other complaints and development was apparently normal. Elsewhere, a diagnosis of Klippel-Trenaunay syndrome had been made and the patient was referred to our department for further evaluation at the age of 12 years.

\section{Physical examination}

Upon examination, both lower legs appeared swollen with a puffy aspect. The swelling was due to a non-pitting oedema with moderate induration of the skin. Palpation was not painful. The palms and soles showed multiple telangiectasias that emptied when compressed and cutis marmorata-like lividity of the skin (Fig. 2). On several toes, small dark-red papular lesions resembling angiomas were seen (Fig. 3). These, too, could be emptied with compression. Some toes appeared erythematous. A receding frontal hairline was noted, with thinly implanted though normal appearing hair. Exclamation mark hairs were not seen. Eyebrows and eyelashes were missing; pubic and axillary hair growth was scant (Fig. 4). There was a slight mongoloid slant of the eyes. Nails and teeth appeared normal and physical examination did not reveal other abnormalities, particularly no cardiac murmurs, short stature, hyperkeratotic skin lesions or pigmentary abnormalities. A paediatric evaluation revealed no abnormalities other than those described above. Skin biopsy was refused. 


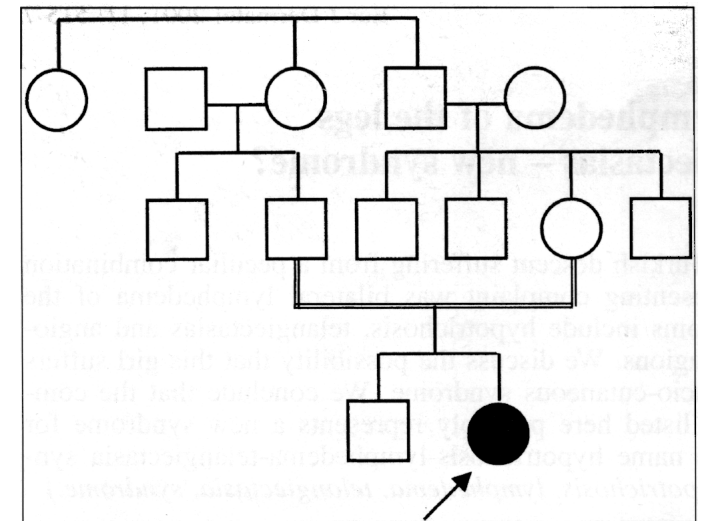

Figure Pedigree of the family.

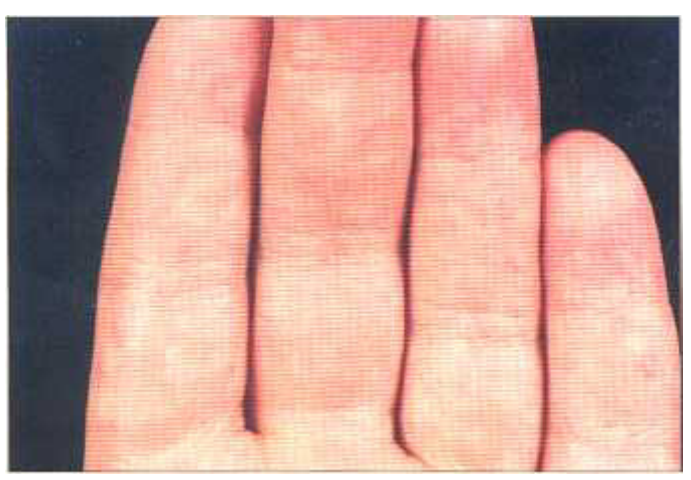

Figure 2. Palmar surface of patient's fingers (right hand). Note lividity and telangiectasias.

\section{Discussion}

The combination of lymphedema and sparse hair is found in the cardio-facio-cutaneous syndrome. This disorder, that is most likely identical to Noonan syndrome [1], is characterised by abnormal (i.e., fine and sparse) hair, hyperkeratotic skin lesions, typical face, short stature, lymphedema and cardiac defects [2]. The symptoms found in our patient show some overlap with CFC syndrome. However, cardiac defects were not found in our patient. Hyperkeratotic lesions or pigmentary abnormalities such as café au lait maculae are not present and neither are overt facial abnormalities. The growth deficiency that almost invariably occurs in CFC/Noonan syndrome [3] is absent as well. Finally, the telangiectasias, angioma-like lesions and cutis marmorata-like skin lividity involving the acral areas are not part of CFC/Noonan syndrome. KlippelTrenaunay syndrome was considered as an explanation for the lymphedema but deemed less likely because large teleangiectatic nevi and overgrowth of limbs were lacking [4].

The hypotrichosis had been present for as long as the patient could remember. Eyelashes had never been present and the eyebrows had always been thinly implanted. The scalp hair did not grow well. There had been no episodes

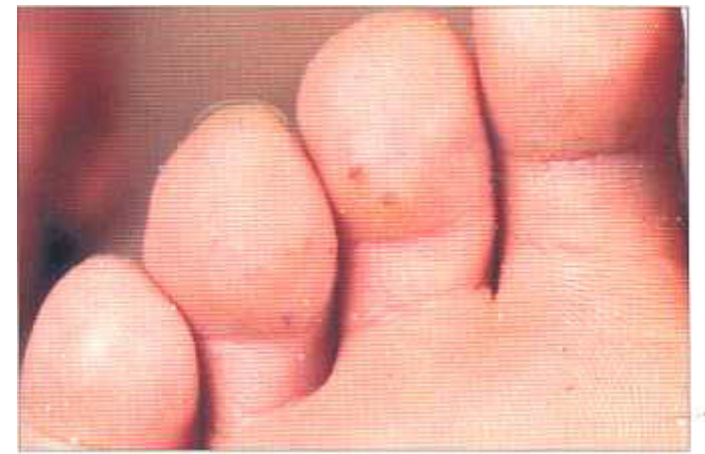

Figure 3. Plantar surface of patient's toes - right foot. Note small angiomatous lesions.

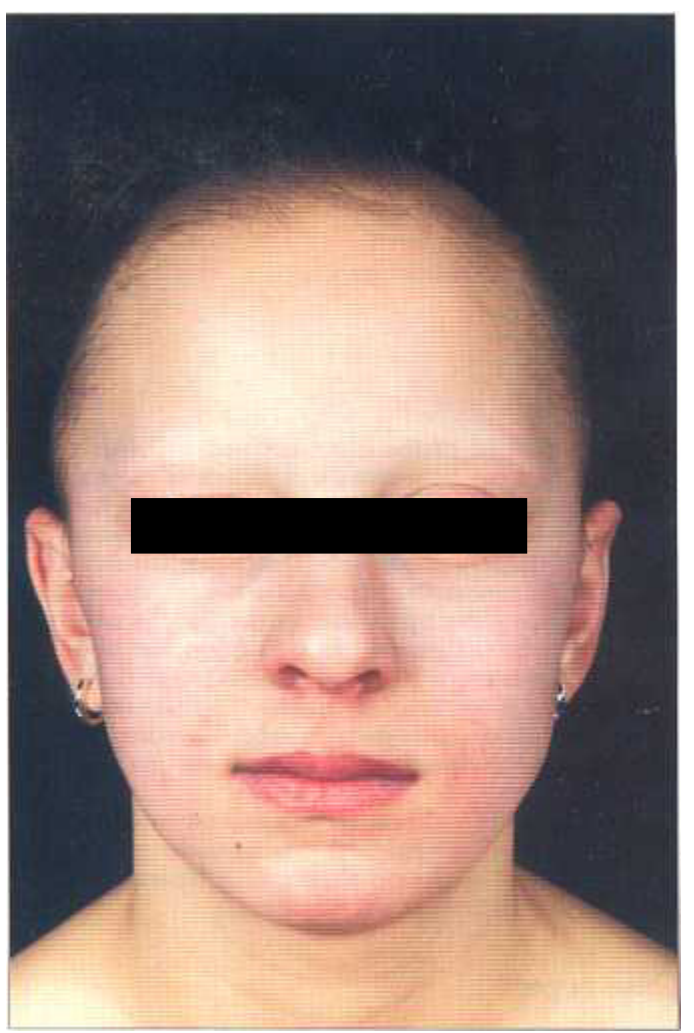

Figure 4. Patient's face, frontal view. High frontal hairline lack of eyebrows and eyelashes.

of accelerated hair loss. Considering this history, alopecia areata as an explanation for the hypotrichosis seems unlikely and congenital hypotrichosis a more appropriate diagnosis. The diffuse pattern and the receded frontal hair line support this notion. 
The lesions on the toes bear some resemblance to lymphangiectases in the context of lymphedema. Other skin signs of lymphedema were missing, however, and the lesions were red as opposed to purple, as lymphangiectases usually are. Moreover, they could be emptied using manual pressure. For these reasons we diagnosed the lesions as angiomata.

In conclusion, we feel that the phenotype we describe here is distinct from CFC/Noonan syndrome despite having overlapping features. We propose that it is a new entity for which we propose the name hypotrichosis-lymphedematelangiectasia syndrome.

\section{Acknowledgements}

M.A.M. van Steensel is supported by grants from the Dutch Organisation for Scientific Research (NOW grant 920-05-083) and Rebirth SA, Luxembourg.

\section{Article accepted on 14/5/01}

\section{References}

1. Leichtman LG. Are cardio-facio-cutaneous syndrome and Noonan syndrome distinct? A case of CFC offspring of a mother with Noonan syndrome. Clin Dysmorphol 1996; 5: 61-4.

2. Reynolds JF, Neri G, Herrmann JP, Blumberg B, Coldwell JG, Miles PV, Opitz JM. New multiple congenital anomalies/mental retardation syndrome with cardio-facio-cutaneous involvement the CFC syndrome. Am J Med Genet 1986; 25: 413-27.

3. Opitz JM. The Noonan syndrome. Am J Med Genet 1985; 4: 333-43. . 4. Samuel M, Spitz L. Klippel-Trenaunay syndrome: clinical features, complications and management in children. $B r J$ Surg 1995; 82: 757-61. 
General discussion 
Molecular biology is a fast-paced field. Any review is quickly outdated; today's hot findings are tomorrow's history. The introduction offered an outline of what is known today and, perhaps more importantly, of what is not. The dominant picture that may well emerge from it is one of daunting complexity. This should not deter us. Many complex systems obey a relatively limited set of rules but can show what is known as "emerging properties" 239 because the number of participants and their interactions is so large. In a way, a biological system is a chaotic one in which the rules do not predict every possible outcome. In order to understand such a system, one only needs to understand the basic rules. Running simulations using these rules will not necessarily duplicate all observed outcomes. It will, however, predict the possible behavioral patterns of the system within given boundary conditions and starting values. The near future is likely to bring an increased, perhaps even complete, understanding of the earliest events in hair follicle morphogenesis. Because the same proteins that coordinate morphogenesis orchestrate hair follicle cycling, an understanding of the adult hair follicle will soon follow. While it is by no means certain that any therapy for acquired or congenital hair loss will soon be available, it seems reasonable to expect that other scientific fields will profit.

Congenital hypotrichoses that closely resemble male pattern baldness are particularly interesting both from a scientific and commercial point of view. Hypotrichosis congenita of Marie Unna (HMU) mimics some key aspects of male pattern baldness and as such presented a natural target for study. The linkage analysis we performed is described in chapter 2. While powerful, linkage is and will remain a black box approach - it enables one to identify a gene locus without any prior knowledge about the potential identity or function of the gene. Identification of a locus does not equal identification of the gene. We have been able to narrow down the HMU locus from the original 1.1 Mb to $350 \mathrm{~kb}$ and screened every gene in it for mutations. None were found. A small gap still remains in the sequence. It is not covered in the 
human genome databases because no BAC clones containing it exist. What is in the gap remains to be determined.

While HMU has not yet delivered as a model system, the study of other disorders has been more fruitful. In chapters 3-9, the results of mutation analysis in a group of related disorders caused by mutations in gap junction genes are described. Gap junctions are intercellular channels that allow the passage of ions and small molecules. They consist of several separate gap junction proteins, or connexins. Gap junctions are instrumental in connecting a group of cells into a functional unit. For example, gap junctions between cardiac myocytes are needed for the conduction of the electric signals that make the heart beat in time. A few years ago, it was rather unexpectedly shown that mutations in certain gap junction genes can cause skin disease. After these first results, several other skin diseases were found to be associated with gap junction gene mutations. We and others have now found that different mutations in a single gap junction gene can cause quite different phenotypes. Keratitis-ichthyosis-deafness (KID) syndrome, a disease that predisposes to skin cancer, can be caused by a mutation that changes glycine 12 of the GJB2 (connexin 26) gap junction protein to an arginine (G12R). A mutation that changes the asparagine at position 14, only 2 amino acids away, to a lysine $(\mathrm{N} 14 \mathrm{~K})$, causes a disease characterized by nail dystrophy and deafness. Apparently, the two different mutations have quite different effects on the eventual GJB2 protein. Intriguingly, our results show that germ line mutations in GJB2 predispose to squamous cell carcinoma (SCC). While it remains to be determined to what extent sporadic mutations in GJB2 can contribute to SCC, preliminary studies in our lab have demonstrated the presence of mutated GJB2 in 15\% of SCC's examined, suggesting that disturbed gap junction communication may have a causal role in skin carcinogenesis. Why and how such mutations can dysregulate growth and differentiation is currently the subject of intensive research.

The diversity of the phenotypes associated with mutations in GJB2 shows that there must be many layers of complexity in the function of connexin 26 . Some 
recent data indicate that GJB2 has functions that are not related to its being part of a gap junction, suggesting that these may be disturbed by some of the mutations that cause skin disease but not by others. However, it is entirely possible that the final explanation will turn out to be even more complex. A possible explanation may be found in the observation that different mutations will affect the conformation of the protein differently. As a result, assembly of the protein into the gap junction will also be abnormal, as will be the gap junction's eventual electrical properties and conductivity to water, ions and small molecules. However, crystal structure is not yet available for any gap junction protein, nor do we have one for an entire gap junction. Hence it is difficult, if not impossible, to predict the effect of mutations on conformation, let alone the effect they will have on gap junction assembly. One direction that future research needs to take is to examine the processes that may be regulated by gap junctions, in addition to the gap junctions themselves. Obtaining reliable crystal structure for a membrane-inserted gap junction protein should have top priority.

Rather than going blindly, it may be wise to go back to the patients first. The diseases tell us what to look for. As an example, many gap junction mutations cause abnormal keratinization. Some mutations in GJB6 can cause a thickening of the nails that is indistinguishable from that caused by some keratin diseases (thesis, chapter 6). The obvious place to start is therefore the regulation of keratin gene expression by gap junctions, focusing on the keratins that are expressed in the nail bed. From other studies it is already known that gap junctions are involved in the conductance of calcium waves through tissues. Calcium is very important in keratinocytes, being involved as a second messenger in the induction of differentiation pathways such as the expression of keratin genes. It is quite possible that some differences between the phenotypes associated with the different mutations may be explained by a differential effect of the mutation on conductivity to calcium and, hence, the process of keratinization. Compare the phenotype of the N14K mutation to KID syndrome and the idea should be quite clear. 
The final question in all medical research is always and will probably always remain the same: what's the use of this research for the patient? The author firmly believes in the principle of "l'art pour l'art". Whether or not research is in any way applicable is not relevant. Knowledge in itself is desirable; those who do not understand that should keep far from research or any other field where an inquisitive mind is essential. It is fashionable nowadays even among those who consider themselves scientists to ask whether knowledge gained is applicable or "socially relevant". For those people it is stated here that the knowledge gained by fundamental and applied genetics research will one day transform medical therapy. In some cases, the transformation is already happening with the introduction of imatinib mesylate (Glivec®) as one of the more dramatic examples. Glivec is targeted agains the tyrosine kinase active site of the BCR-ABL fusion protein that is causes the unchecked cell growth in chronic myeloid leukemia 240,241. Growth of the leukemic cells is inhibited while normal tyrosine kinase signaling remains mostly intact. Other tyrosine kinase inhibitors such as gefitinib mesylate (inhibiting the epidermal growth factor receptor, a tyrosine kinase), also known as Iressa®, show great promise for the treatment of solid tumors such as small cell lung carcinoma ${ }^{242}$. A very obvious benefit meanwhile is the increased accuracy of disease classification when assisted by molecular diagnostics. An accurate diagnosis is essential for genetic counseling, prognosis and, increasingly, for choosing an appropriate medical intervention. Again, the gap junction diseases provide a good example where it is clear that very diverse phenotypes can all be caused by mutations in GJB2. The identification of GJB3 and GJB4 mutations in erythrokeratoderma variabilis (EKV) is proving to be very useful in delineating EKV from diseases that can resemble it such as non-bullous ichthyosiform erythroderma, a disease requiring different clinical management $^{243}$.

Once we understand the why, how and when of the way our bodies function down to the protein level we will be able to fix them when dysfunctional. In that respect, a biological machine does not differ significantly from a car or 
any other complex mechanical construction. One example is described in chapter 10, where we analyzed the plakophilin-1 gene in a patient with McGrath syndrome. This disorder causes a severe defect in the adhesion between skin cells to such a degree that even slight trauma will result in a loss of cohesion. Erosions are the consequence. On the palms and soles, the skin attempts to repair itself and massive hyperkeratosis ensues. The fragility remains, however, resulting in painful fissuring. Most patients cannot walk because of it. The hair is lost because the follicles do not adhere to the surrounding skin. It's a severe disease, of which our patient had a curiously mild manifestation. He could still walk and had most of his hair, although it was oddly curly and could be painlessly removed by pulling firmly. Mutation analysis showed a novel splice donor site mutation that should normally result in defective splicing of the gene and production of a non-functional protein. Our patient's splicing machinery, however, made use of a so-called cryptic splice donor site (a site that is otherwise hidden from the splicing riboproteins), enabling the production of an almost correct messenger RNA. From that a nearly full length protein is produced. It lacks part of an essential DNA binding domain and is not fully functional but it serves to ameliorate the disease. Our finding suggests that one day, it may be possible to ameliorate or correct genetic disorders caused by aberrant splicing. For some disorders it has already been demonstrated that administration of certain antibiotics can help to correct splicing defects, thus improving clinical symptoms 244,245 .

We see how Nature offers up clues for the scientist. The clinical scientist finds his or herself in a true Bonanza. Every disease contains a lesson, as shown in this thesis. Of particular interest are the "new" monogenic diseases, as these point to genes that have not yet been characterized, while at the same time showing what the consequences of dysfunction of that particular gene are. Careful analysis of the phenotype is necessary in order to get an idea of what the gene function may be, and of whether or not one really has to do with a new disease. In chapters 11-15, clinical studies in one extremely rare and three 
new monogenic syndromes are described. The finding of a SOX18 mutation in patients with the hypotrichosis-lymphedema-telangiectasia syndrome ${ }^{224}$ proves that identification of rare disorders can and will lead to the identification of important "new" developmental genes. 


\section{Future directions}

The future will doubtlessly bring some more new genes, many more new proteins and ever more questions. The way things are, we will probably run out of new human disease genes to discover in the next ten years or so. The next challenge is in systems biology, that is, the understanding of how the various proteins interact. Clunky terms such as "metabolomics", "transcriptomics" and "proteomics" have been coined in the past but "systems biology" seems a more accurate description considering the subject. One of the key problems to tackle will be the massive amount of potential interactions in protein networks. The basic rules are relatively simple (binding is binding, regardless of the nature of the phenomenon at the atomary level) but the results are not. In order to simulate emerging properties, all interactions will have to be modeled. While considerable progress is being made in particular with relatively simple organisms such as baker's yeast ${ }^{246}$ it will not be easy to extrapolate those data to more complex organisms such as insects or mammals. In the near future, it will probably become possible and feasible to model an entire yeast proteome and confidently predict the outcome of perturbations in the yeast protein network. For more complex organisms, one might envision that interactions within functional modules such as the cell cycle machinery will be modeled first and then verified using knock-out technology or comparable approaches. The emerging technology of RNA interference holds great promise in that regard, enabling highthroughput knock-down of target genes ${ }^{247}$. If succesfull, the process can be scaled up to include more modules and the interactions between them. This problem, contrary to what may be thought, will not be NP-hard or -complete. Once an individual module has been fully modeled it may be treated as a black box as far as the inter-module network is concerned. The ideal is obviously to be able to model all protein interactions in silico without needing access to the organism of interest itself, unless perhaps for verification of 
results. The computational requirements however exceed those that are currently available to most life scientists. It is thus quite probable that computational biology using "big iron" "*ill become one of the hottest fields, simply because we will all desperately need it to understand what a given protein network is doing. The problem becomes even more acute when considering pharmacology. High-throughput drug screening and rational drug design will give us a great many new interesting and promising ways in which to influence life, but there is no way that we are ever going to be able to test each and every new interesting drug in live systems. Thus, it is important that biological scientists be aware of and informed about the possibilities that current computer technology offers for the construction of relatively inexpensive big iron such as Beowulf clusters ${ }^{248}$. Apple Computer hardware currently offers the most processing power per US dollar spent on it.

Time to start saving up for a nice G5 cluster!

\footnotetext{
${ }^{*}$ Hacker term denoting large (super)computers or clusters
} 
References 
1 Gianoutsos P. Heed Yul Brynner. Med J Aust 1987; 146: 288-9.

2 Lee HJ, Ha SJ, Kim D et al. Perception of men with androgenetic alopecia by women and nonbalding men in Korea: how the nonbald regard the bald. Int $J$ Dermatol 2002; 41: 867-9.

3 Storer JS, Brzuskiewicz J, Floyd H et al. Topical minoxidil for male pattern baldness. Am J Med Sci 1986; 291: 328-33.

4 Cash TF. The psychosocial consequences of androgenetic alopecia: a review of the research literature. Br J Dermatol 1999; 141: 398-405.

$5 \quad$ Trachet T. Skepter 1999; 12: 6-9.

$6 \quad$ Paget J. Lancet 1882; 2: 1107.

7 van Steensel MA, van Geel M, Steiljen PM. The molecular basis of hair growth. Eur J Dermatol 2001; 11: 348-52.

8 Van Steensel MA, Happle R, Steijlen PM. Molecular genetics of the hair follicle: the state of the art. Proc Soc Exp Biol Med 2000; 223: 1-7.

$9 \quad$ van Steensel MA. Gap junction diseases of the skin. Am J Med Genet 2004; 131C: $12-9$.

10 van Steensel MA, van Geel M, Steijlen PM. Molecular genetics of hereditary hair and nail disease. Am J Med Genet 2004; 131C: 52-60.

11 Jamora C, DasGupta R, Kocieniewski P et al. Links between signal transduction, transcription and adhesion in epithelial bud development. Nature 2003; 422: 317-22.

12 Paus R. Principles of hair cycle control. J Dermatol 1998; 25: 793-802.

13 Milner Y, Sudnik J, Filippi M et al. Exogen, shedding phase of the hair growth cycle: characterization of a mouse model. J Invest Dermatol 2002; 119: 63944.

14 Gambardella L, Barrandon Y. The multifaceted adult epidermal stem cell. Curr Opin Cell Biol 2003; 15: 771-7.

15 Frank J, Pignata C, Panteleyev AA et al. Exposing the human nude phenotype. Nature 1999; 398: 473-4.

16 Stenn KS, Paus R. Controls of hair follicle cycling. Physiol Rev 2001; 81: 449-94.

17 Powell BC, Passmore EA, Nesci A et al. The Notch signalling pathway in hair growth. Mech Dev 1998; 78: 189-92. 
18 Pourquie O. Skin development: delta laid bare. Curr Biol 2000; 10: R425-8.

19 Dale KJ, Pourquie O. A clock-work somite. Bioessays 2000; 22: 72-83.

20 Hardy MH. The secret life of the hair follicle. Trends Genet 1992; 8: 55-61.

21 Hogan BL. Morphogenesis. Cell 1999; 96: 225-33.

22 Botchkarev VA, Botchkareva NV, Roth W et al. Noggin is a mesenchymally derived stimulator of hair-follicle induction. Nat Cell Biol 1999; 1: 158-64.

23 van Genderen C, Okamura RM, Farinas I et al. Development of several organs that require inductive epithelial-mesenchymal interactions is impaired in LEF1-deficient mice. Genes Dev 1994; 8: 2691-703.

24 Zhou P, Byrne C, Jacobs J et al. Lymphoid enhancer factor 1 directs hair follicle patterning and epithelial cell fate. Genes Dev 1995; 9: 700-13.

25 Gat U, DasGupta R, Degenstein L et al. De Novo hair follicle morphogenesis and hair tumors in mice expressing a truncated beta-catenin in skin. Cell 1998; 95: 605-14.

26 DasGupta R, Fuchs E. Multiple roles for activated LEF/TCF transcription complexes during hair follicle development and differentiation. Development 1999; 126: 4557-68.

27 Huelsken J, Vogel R, Erdmann B et al. beta-Catenin controls hair follicle morphogenesis and stem cell differentiation in the skin. Cell 2001; 105: 53345.

28 Hirai Y, Nose A, Kobayashi S et al. Expression and role of E- and P-cadherin adhesion molecules in embryonic histogenesis. II. Skin morphogenesis. Development 1989; 105: 271-7.

29 Rijsewijk F, Schuermann M, Wagenaar E et al. The Drosophila homolog of the mouse mammary oncogene int- 1 is identical to the segment polarity gene wingless. Cell 1987; 50: 649-57.

30 McMahon AP, Moon RT. int-1--a proto-oncogene involved in cell signalling. Development 1989; 107 Suppl: 161-7.

31 Nusse R, Varmus HE. Many tumors induced by the mouse mammary tumor virus contain a provirus integrated in the same region of the host genome. Cell 1982; 31: 99-109.

32 Dickson C, Smith R, Brookes S et al. Tumorigenesis by mouse mammary tumor virus: proviral activation of a cellular gene in the common integration region int-2. Cell 1984; 37: 529-36. 
33 Gumbiner BM. Regulation of cadherin adhesive activity. J Cell Biol 2000; 148: $399-404$.

34 Daniels DL, Eklof Spink K, Weis WI. beta-catenin: molecular plasticity and drug design. Trends Biochem Sci 2001; 26: 672-8.

35 Gumbiner BM, McCrea PD. Catenins as mediators of the cytoplasmic functions of cadherins. J Cell Sci Suppl 1993; 17: 155-8.

36 Hulsken J, Birchmeier W, Behrens J. E-cadherin and APC compete for the interaction with beta-catenin and the cytoskeleton. J Cell Biol 1994; 127: 2061-9.

37 Rubinfeld B, Albert I, Porfiri E et al. Binding of GSK3beta to the APC-betacatenin complex and regulation of complex assembly. Science 1996; 272: 1023-6.

38 Hamada F, Tomoyasu Y, Takatsu Y et al. Negative regulation of Wingless signaling by D-axin, a Drosophila homolog of axin. Science 1999; 283: 173942.

39 Behrens J. Control of beta-catenin signaling in tumor development. Ann N Y Acad Sci 2000; 910: 21-33; discussion -5.

40 Aberle $\mathrm{H}$, Bauer A, Stappert J et al. beta-catenin is a target for the ubiquitinproteasome pathway. Embo J 1997; 16: 3797-804.

41 Bhanot $\mathrm{P}$, Brink $\mathrm{M}$, Samos $\mathrm{CH}$ et al. A new member of the frizzled family from Drosophila functions as a Wingless receptor. Nature 1996; 382: 225-30.

42 Itoh K, Antipova A, Ratcliffe MJ et al. Interaction of dishevelled and Xenopus axin-related protein is required for wnt signal transduction. Mol Cell Biol 2000; 20: 2228-38.

43 Molenaar M, van de Wetering M, Oosterwegel M et al. XTcf-3 transcription factor mediates beta-catenin-induced axis formation in Xenopus embryos. Cell 1996; 86: 391-9.

44 van Steensel MA, Celli J, van Bokhoven JH et al. Probing the gene expression database for candidate genes. Eur J Hum Genet 1999; 7: 910-9.

45 Picard C, Couderc S, Skojaei T et al. Scalp-ear-nipple (Finlay-Marks) syndrome: a familial case with renal involvement. Clin Genet 1999; 56: 170-2.

46 Edwards MJ, McDonald D, Moore P et al. Scalp-ear-nipple syndrome: additional manifestations. Am J Med Genet 1994; 50: 247-50. 
47 Sonoda T. [Scalp-ear-nipple syndrome]. Ryoikibetsu Shokogun Shirizu 2001: 606-7.

48 Kratochwil K, Dull M, Farinas I et al. Lef1 expression is activated by BMP-4 and regulates inductive tissue interactions in tooth and hair development. Genes Dev 1996; 10: 1382-94.

49 Mikkola ML, Pispa J, Pekkanen M et al. Ectodysplasin, a protein required for epithelial morphogenesis, is a novel TNF homologue and promotes cell-matrix adhesion. Mech Dev 1999; 88: 133-46.

50 Headon DJ, Overbeek PA. Involvement of a novel Tnf receptor homologue in hair follicle induction. Nat Genet 1999; 22: 370-4.

51 Monreal AW, Ferguson BM, Headon DJ et al. Mutations in the human homologue of mouse dl cause autosomal recessive and dominant hypohidrotic ectodermal dysplasia. Nat Genet 1999; 22: 366-9.

52 Yan M, Wang LC, Hymowitz SG et al. Two-amino acid molecular switch in an epithelial morphogen that regulates binding to two distinct receptors. Science 2000; 290: 523-7.

53 Doffinger R, Smahi A, Bessia C et al. X-linked anhidrotic ectodermal dysplasia with immunodeficiency is caused by impaired NF-kappaB signaling. Nat Genet 2001; 27: 277-85.

54 Milligan SA, Owens MW, Grisham MB. Inhibition of IkappaB-alpha and IkappaB-beta proteolysis by calpain inhibitor I blocks nitric oxide synthesis. Arch Biochem Biophys 1996; 335: 388-95.

55 Natoli G, Costanzo A, Guido F et al. Apoptotic, non-apoptotic, and antiapoptotic pathways of tumor necrosis factor signalling. Biochem Pharmacol 1998; 56: 915-20.

56 Ferguson BM, Brockdorff N, Formstone E et al. Cloning of Tabby, the murine homolog of the human EDA gene: evidence for a membrane-associated protein with a short collagenous domain. Hum Mol Genet 1997; 6: 1589-94.

57 Kovalenko A, Chable-Bessia C, Cantarella G et al. The tumour suppressor CYLD negatively regulates NF-kappaB signalling by deubiquitination. Nature 2003; 424: 801-5.

58 Brummelkamp TR, Nijman SM, Dirac AM et al. Loss of the cylindromatosis tumour suppressor inhibits apoptosis by activating NF-kappaB. Nature 2003; 424: 797-801. 
59 Trompouki E, Hatzivassiliou E, Tsichritzis T et al. CYLD is a deubiquitinating enzyme that negatively regulates NF-kappaB activation by TNFR family members. Nature 2003; 424: 793-6.

60 Bignell GR, Warren W, Seal S et al. Identification of the familial cylindromatosis tumour-suppressor gene. Nat Genet 2000; 25: 160-5.

61 Makris C, Roberts JL, Karin M. The carboxyl-terminal region of IkappaB kinase gamma (IKKgamma) is required for full IKK activation. Mol Cell Biol 2002; 22: 6573-81.

62 Kere J, Srivastava AK, Montonen O et al. X-linked anhidrotic (hypohidrotic) ectodermal dysplasia is caused by mutation in a novel transmembrane protein. Nat Genet 1996; 13: 409-16.

63 Headon DJ, Emmal SA, Ferguson BM et al. Gene defect in ectodermal dysplasia implicates a death domain adapter in development. Nature 2001; 414: 913-6.

64 Isaacs K, Brown G, Moore GP. Interactions between epidermal growth factor and the Tabby mutation in skin. Exp Dermatol 1998; 7: 273-80.

65 Yoon YM, Oh CD, Kim DY et al. Epidermal growth factor negatively regulates chondrogenesis of mesenchymal cells by modulating the protein kinase C-alpha, Erk-1, and p38 MAPK signaling pathways. J Biol Chem 2000; 275: 12353-9.

66 Read MA, Whitley MZ, Gupta S et al. Tumor necrosis factor alpha-induced Eselectin expression is activated by the nuclear factor-kappaB and c-JUN Nterminal kinase/p38 mitogen-activated protein kinase pathways. J Biol Chem 1997; 272: 2753-61.

67 Smahi A, Courtois G, Vabres P et al. Genomic rearrangement in NEMO impairs NF-kappaB activation and is a cause of incontinentia pigmenti. The International Incontinentia Pigmenti (IP) Consortium. Nature 2000; 405: 46672.

68 Laurikkala J, Pispa J, Jung HS et al. Regulation of hair follicle development by the TNF signal ectodysplasin and its receptor Edar. Development 2002; 129: 2541-53.

69 Yu M, Wu P, Widelitz RB et al. The morphogenesis of feathers. Nature 2002; 420: 308-12. 
70 Iseki S, Araga A, Ohuchi H et al. Sonic hedgehog is expressed in epithelial cells during development of whisker, hair, and tooth. Biochem Biophys Res Commun 1996; 218: 688-93.

71 Widelitz RB, Jiang TX, Chen CW et al. Wnt-7a in feather morphogenesis: involvement of anterior-posterior asymmetry and proximal-distal elongation demonstrated with an in vitro reconstitution model. Development 1999; 126: 2577-87.

72 Vervoort M. hedgehog and wing development in Drosophila: a morphogen at work? Bioessays 2000; 22: 460-8.

73 Ellis T, Gambardella L, Horcher $\mathrm{M}$ et al. The transcriptional repressor CDP (Cutl1) is essential for epithelial cell differentiation of the lung and the hair follicle. Genes Dev 2001; 15: 2307-19.

74 St-Jacques B, Dassule HR, Karavanova I et al. Sonic hedgehog signaling is essential for hair development. Curr Biol 1998; 8: 1058-68.

75 Chiang C, Swan RZ, Grachtchouk M et al. Essential role for Sonic hedgehog during hair follicle morphogenesis. Dev Biol 1999; 205: 1-9.

76 Mill P, Mo R, Fu H et al. Sonic hedgehog-dependent activation of Gli2 is essential for embryonic hair follicle development. Genes Dev 2003; 17: 28294.

77 Marigo V, Davey RA, Zuo Y et al. Biochemical evidence that patched is the Hedgehog receptor. Nature 1996; 384: 176-9.

78 Alcedo J, Ayzenzon M, Von Ohlen T et al. The Drosophila smoothened gene encodes a seven-pass membrane protein, a putative receptor for the hedgehog signal. Cell 1996; 86: 221-32.

79 Stone DM, Hynes M, Armanini M et al. The tumour-suppressor gene patched encodes a candidate receptor for Sonic hedgehog. Nature 1996; 384: 129-34.

80 Hahn H, Christiansen J, Wicking C et al. A mammalian patched homolog is expressed in target tissues of sonic hedgehog and maps to a region associated with developmental abnormalities. J Biol Chem 1996; 271: 12125-8.

81 Hahn H, Wicking C, Zaphiropoulous PG et al. Mutations of the human homolog of Drosophila patched in the nevoid basal cell carcinoma syndrome. Cell 1996; 85: 841-51. 
82 Nilsson M, Unden AB, Krause D et al. Induction of basal cell carcinomas and trichoepitheliomas in mice overexpressing GLI-1. Proc Natl Acad Sci U S A 2000; 97: 3438-43.

83 Bumcrot DA, Takada R, McMahon AP. Proteolytic processing yields two secreted forms of sonic hedgehog. Mol Cell Biol 1995; 15: 2294-303.

84 Lopez-Martinez A, Chang DT, Chiang C et al. Limb-patterning activity and restricted posterior localization of the amino-terminal product of Sonic hedgehog cleavage. Curr Biol 1995; 5: 791-6.

85 Porter JA, von Kessler DP, Ekker SC et al. The product of hedgehog autoproteolytic cleavage active in local and long-range signalling. Nature 1995; 374: 363-6.

86 Fietz MJ, Jacinto A, Taylor AM et al. Secretion of the amino-terminal fragment of the hedgehog protein is necessary and sufficient for hedgehog signalling in Drosophila. Curr Biol 1995; 5: 643-50.

87 Porter JA, Ekker SC, Park WJ et al. Hedgehog patterning activity: role of a lipophilic modification mediated by the carboxy-terminal autoprocessing domain. Cell 1996; 86: 21-34.

88 Porter JA, Young KE, Beachy PA. Cholesterol modification of hedgehog signaling proteins in animal development. Science 1996; 274: 255-9.

89 Happle R. X-linked dominant chondrodysplasia punctata. Review of literature and report of a case. Hum Genet 1979; 53: 65-73.

90 Kelley RI, Wilcox WG, Smith M et al. Abnormal sterol metabolism in patients with Conradi-Hunermann-Happle syndrome and sporadic lethal chondrodysplasia punctata. Am J Med Genet 1999; 83: 213-9.

91 Derry JM, Gormally E, Means GD et al. Mutations in a delta 8-delta 7 sterol isomerase in the tattered mouse and X-linked dominant chondrodysplasia punctata. jderry@immunex.com. Nat Genet 1999; 22: 286-90.

92 Traupe H, Has C. The Conradi-Hunermann-Happle syndrome is caused by mutations in the gene that encodes a 8- 7 sterol isomerase and is biochemically related to the CHILD syndrome. Eur J Dermatol 2000; 10: 425-8.

93 Happle R. Mosaicism in human skin. Understanding the patterns and mechanisms. Arch Dermatol 1993; 129: 1460-70. 
94 Konig A, Happle R, Bornholdt D et al. Mutations in the NSDHL gene, encoding a 3beta-hydroxysteroid dehydrogenase, cause CHILD syndrome. Am J Med Genet 2000; 90: 339-46.

95 Happle R, Koch H, Lenz W. The CHILD syndrome. Congenital hemidysplasia with ichthyosiform erythroderma and limb defects. Eur J Pediatr 1980; 134: 27-33.

96 Levin M. Left-right asymmetry in vertebrate embryogenesis. Bioessays 1997; 19: 287-96.

97 Zhang XM, Lin E, Yang XJ. Sonic hedgehog-mediated ventralization disrupts formation of the midbrain-hindbrain junction in the chick embryo. Dev Neurosci 2000; 22: 207-16.

98 Loomis CA, Harris E, Michaud J et al. The mouse Engrailed-1 gene and ventral limb patterning. Nature 1996; 382: 360-3.

99 Briscoe J, Chen Y, Jessell TM et al. A hedgehog-insensitive form of patched provides evidence for direct long-range morphogen activity of sonic hedgehog in the neural tube. Mol Cell 2001; 7: 1279-91.

100 Randall VA, Ebling FJ. Seasonal changes in human hair growth. $\mathrm{Br} J$ Dermatol 1991; 124: 146-51.

101 Macias-Flores MA, Garcia-Cruz D, Rivera H et al. A new form of hypertrichosis inherited as an X-linked dominant trait. Hum Genet 1984; 66: 66-70.

102 Figuera LE, Pandolfo M, Dunne PW et al. Mapping of the congenital generalized hypertrichosis locus to chromosome Xq24-q27.1. Nat Genet 1995; 10: $202-7$.

103 Foitzik K, Krause K, Nixon AJ et al. Prolactin and its receptor are expressed in murine hair follicle epithelium, show hair cycle-dependent expression, and induce catagen. Am J Pathol 2003; 162: 1611-21.

104 Craven AJ, Ormandy CJ, Robertson FG et al. Prolactin signaling influences the timing mechanism of the hair follicle: analysis of hair growth cycles in prolactin receptor knockout mice. Endocrinology 2001; 142: 2533-9.

105 Freeman ME, Kanyicska B, Lerant A et al. Prolactin: structure, function, and regulation of secretion. Physiol Rev 2000; 80: 1523-631. 
106 Smale L, Nelson RJ, Zucker I. Daylength influences pelage and plasma prolactin concentrations but not reproduction in the prairie vole, Microtus ochrogaster. J Reprod Fertil 1988; 83: 99-106.

107 Lincoln GA. Correlation with changes in horns and pelage, but not reproduction, of seasonal cycles in the secretion of prolactin in rams of wild, feral and domesticated breeds of sheep. J Reprod Fertil 1990; 90: 285-96.

108 Nixon AJ, Ford CA, Wildermoth JE et al. Regulation of prolactin receptor expression in ovine skin in relation to circulating prolactin and wool follicle growth status. J Endocrinol 2002; 172: 605-14.

109 Moreno-Arias G, Castelo-Branco C, Ferrando J. Paradoxical effect after IPL photoepilation. Dermatol Surg 2002; 28: 1013-6; discussion 6.

110 Kuhlmann MT, Clemen G, Schlatt S. Molting in the Djungarian hamster (Phodopus sungorus Pallas): Seasonal or continuous process? J Exp Zool 2003; 295A: 160-71.

111 Gebbie FE, Forsyth IA, Arendt J. Effects of maintaining solstice light and temperature on reproductive activity, coat growth, plasma prolactin and melatonin in goats. J Reprod Fertil 1999; 116: 25-33.

112 Rose J, Kennedy M, Johnston B et al. Serum prolactin and dehydroepiandrosterone concentrations during the summer and winter hair growth cycles of mink (Mustela vison). Comp Biochem Physiol A Mol Integr Physiol 1998; 121: 263-71.

113 Choy VJ, Nixon AJ, Pearson AJ. Distribution of prolactin receptor immunoreactivity in ovine skin and changes during the wool follicle growth cycle. J Endocrinol 1997; 155: 265-75.

114 Martinet L, Allain D, Weiner C. Role of prolactin in the photoperiodic control of moulting in the mink (Mustela vison). J Endocrinol 1984; 103: 9-15.

115 Walsh JP, Pullan PT. Hyperprolactinaemia in males: a heterogeneous disorder. Aust N Z J Med 1997; 27: 385-90.

116 Orfanos CE, Hertel H. [Disorder of hair growth in hyperprolactinemia]. Z Hautkr 1988; 63: 23-6.

117 Xiao Y, Forsberg M, Laitinen JT et al. Effects of melatonin implants in spring on testicular regression and moulting in adult male raccoon dogs (Nyctereutes procynoides). J Reprod Fertil 1995; 105: 9-15. 
118 Nixon AJ, Choy VJ, Parry AL et al. Fiber growth initiation in hair follicles of goats treated with melatonin. J Exp Zool 1993; 267: 47-56.

119 Paus R, Stenn KS, Link RE. Telogen skin contains an inhibitor of hair growth. Br J Dermatol 1990; 122: 777-84.

120 Botchkarev VA, Botchkareva NV, Nakamura M et al. Noggin is required for induction of the hair follicle growth phase in postnatal skin. Faseb $J$ 2001; 15: 2205-14.

121 Sato N, Leopold PL, Crystal RG. Effect of adenovirus-mediated expression of Sonic hedgehog gene on hair regrowth in mice with chemotherapy-induced alopecia. J Natl Cancer Inst 2001; 93: 1858-64.

122 Sato N, Leopold PL, Crystal RG. Induction of the hair growth phase in postnatal mice by localized transient expression of Sonic hedgehog. J Clin Invest 1999; 104: 855-64.

123 Ebling FJ. Hair. J Invest Dermatol 1976; 67: 98-105.

124 Matsuo K, Mori O, Hashimoto T. Plucking during telogen induces apoptosis in the lower part of hair follicles. Arch Dermatol Res 2003; 295: 33-7.

125 Chase HB, Eaton GJ. The growth of hair follicles in waves. Ann N Y Acad Sci 1959; 83: 365-8.

126 Botchkarev VA, Botchkarev NV, Albers KM et al. Neurotrophin-3 involvement in the regulation of hair follicle morphogenesis. $J$ Invest Dermatol 1998; 111: 279-85.

127 Bjarnason GA, Jordan RC, Wood PA et al. Circadian expression of clock genes in human oral mucosa and skin: association with specific cell-cycle phases. Am J Pathol 2001; 158: 1793-801.

128 Zanello SB, Jackson DM, Holick MF. Expression of the circadian clock genes clock and period1 in human skin. J Invest Dermatol 2000; 115: 757-60.

129 Jaeger J, Goodwin BC. Cellular oscillators in animal segmentation. In Silico Biol 2002; 2: 111-23.

130 Pourquie O. The vertebrate segmentation clock. J Anat 2001; 199: 169-75.

131 Maroto M, Pourquie O. A molecular clock involved in somite segmentation. Curr Top Dev Biol 2001; 51: 221-48.

132 Jaeger J, Goodwin BC. A cellular oscillator model for periodic pattern formation. $J$ Theor Biol 2001; 213: 171-81. 
133 Hirsinger E, Jouve C, Dubrulle J et al. Somite formation and patterning. Int Rev Cytol 2000; 198: 1-65.

134 Jiang YJ, Aerne BL, Smithers L et al. Notch signalling and the synchronization of the somite segmentation clock. Nature 2000; 408: 475-9.

135 Jouve C, Palmeirim I, Henrique D et al. Notch signalling is required for cyclic expression of the hairy-like gene HES1 in the presomitic mesoderm.

Development 2000; 127: 1421-9.

136 Aulehla A, Wehrle C, Brand-Saberi B et al. Wnt3a plays a major role in the segmentation clock controlling somitogenesis. Dev Cell 2003; 4: 395-406.

137 Ahmad W, Irvine AD, Lam $\mathrm{H}$ et al. A missense mutation in the zinc-finger domain of the human hairless gene underlies congenital atrichia in a family of Irish travellers. Am J Hum Genet 1998; 63: 984-91.

138 Ahmad W, Faiyaz ul Haque M, Brancolini V et al. Alopecia universalis associated with a mutation in the human hairless gene. Science 1998; 279: 720-4.

139 Panteleyev AA, Botchkareva NV, Sundberg JP et al. The role of the hairless (hr) gene in the regulation of hair follicle catagen transformation. Am J Pathol 1999; 155: 159-71.

140 Panteleyev AA, Paus R, Christiano AM. Patterns of hairless (hr) gene expression in mouse hair follicle morphogenesis and cycling. Am J Pathol 2000; 157: 1071-9.

141 Potter GB, Beaudoin GM, 3rd, DeRenzo CL et al. The hairless gene mutated in congenital hair loss disorders encodes a novel nuclear receptor corepressor. Genes Dev 2001; 15: 2687-701.

142 Potter GB, Zarach JM, Sisk JM et al. The thyroid hormone-regulated corepressor hairless associates with histone deacetylases in neonatal rat brain. Mol Endocrinol 2002; 16: 2547-60.

143 Davie JR, Spencer VA. Control of histone modifications. J Cell Biochem 1999; Suppl 32-33: 141-8.

144 Miller J, Djabali K, Chen T et al. Atrichia caused by mutations in the vitamin $\mathrm{D}$ receptor gene is a phenocopy of generalized atrichia caused by mutations in the hairless gene. J Invest Dermatol 2001; 117: 612-7. 
145 Hsieh JC, Sisk JM, Jurutka PW et al. Physical and functional interaction between the vitamin D receptor and hairless corepressor, two proteins required for hair cycling. J Biol Chem 2003; 278: 38665-74.

146 Murone M, Rosenthal A, de Sauvage FJ. Sonic hedgehog signaling by the patched-smoothened receptor complex. Curr Biol 1999; 9: 76-84.

147 Foitzik K, Lindner G, Mueller-Roever S et al. Control of murine hair follicle regression (catagen) by TGF-beta1 in vivo. Faseb $J$ 2000; 14: 752-60.

148 Paus R, Foitzik K, Welker P et al. Transforming growth factor-beta receptor type I and type II expression during murine hair follicle development and cycling. J Invest Dermatol 1997; 109: 518-26.

149 Yano K, Brown LF, Lawler J et al. Thrombospondin-1 plays a critical role in the induction of hair follicle involution and vascular regression during the catagen phase. J Invest Dermatol 2003; 120: 14-9.

150 Maurer M, Fischer E, Handjiski B et al. Activated skin mast cells are involved in murine hair follicle regression (catagen). Lab Invest 1997; 77: 319-32.

151 Slepchenko BM, Schaff JC, Carson JH et al. Computational cell biology: spatiotemporal simulation of cellular events. Annu Rev Biophys Biomol Struct 2002; 31: 423-41.

152 Hebert JM, Rosenquist T, Gotz J et al. FGF5 as a regulator of the hair growth cycle: evidence from targeted and spontaneous mutations. Cell 1994; 78: 1017-25.

153 Sundberg JP, Rourk MH, Boggess D et al. Angora mouse mutation: altered hair cycle, follicular dystrophy, phenotypic maintenance of skin grafts, and changes in keratin expression. Vet Pathol 1997; 34: 171-9.

154 De Luca F, Baron J. Control of Bone Growth by Fibroblast Growth Factors. Trends Endocrinol Metab 1999; 10: 61-5.

155 Tavormina PL, Shiang R, Thompson LM et al. Thanatophoric dysplasia (types I and II) caused by distinct mutations in fibroblast growth factor receptor 3. Nat Genet 1995; 9: 321-8.

156 Bellus GA, McIntosh I, Smith EA et al. A recurrent mutation in the tyrosine kinase domain of fibroblast growth factor receptor 3 causes hypochondroplasia. Nat Genet 1995; 10: 357-9. 
157 Shiang R, Thompson LM, Zhu YZ et al. Mutations in the transmembrane domain of FGFR3 cause the most common genetic form of dwarfism, achondroplasia. Cell 1994; 78: 335-42.

158 Rousseau F, Bonaventure J, Legeai-Mallet L et al. Mutations in the gene encoding fibroblast growth factor receptor-3 in achondroplasia. Nature 1994; 371: 252-4.

159 Munro CS, Wilkie AO. Epidermal mosaicism producing localised acne: somatic mutation in FGFR2. Lancet 1998; 352: 704-5.

160 Rees J. FGFR2 mutations and acne. Lancet 1998; 352: 668-9.

161 Salser SJ, Loer CM, Kenyon C. Multiple HOM-C gene interactions specify cell fates in the nematode central nervous system. Genes Dev 1993; 7: 171424.

162 Polani PE. Developmental asymmetries in experimental animals. Neurosci Biobehav Rev 1996; 20: 645-9.

163 Goulding MD, Gruss P. The homeobox in vertebrate development. Curr Opin Cell Biol 1989; 1: 1088-93.

164 Dickman S. HOX gene links limb, genital defects. Science 1997; 275: 1568.

165 Scott MP. Hox genes, arms and the man. Nat Genet 1997; 15: 117-8.

166 Mortlock DP, Innis JW. Mutation of HOXA13 in hand-foot-genital syndrome. Nat Genet 1997; 15: 179-80.

167 Godwin AR, Capecchi MR. Hoxc13 mutant mice lack external hair. Genes Dev 1998; 12: 11-20.

168 Jave-Suarez LF, Winter H, Langbein L et al. HOXC13 is involved in the regulation of human hair keratin gene expression. J Biol Chem 2002; 277: 3718-26.

169 van der Hoeven F, Zakany J, Duboule D. Gene transpositions in the HoxD complex reveal a hierarchy of regulatory controls. Cell 1996; 85: 1025-35.

170 Patterson KD, Cleaver O, Gerber WV et al. Homeobox genes in cardiovascular development. Curr Top Dev Biol 1998; 40: 1-44.

171 Grosser BI, Monti-Bloch L, Jennings-White C et al. Behavioral and electrophysiological effects of androstadienone, a human pheromone. Psychoneuroendocrinology 2000; 25: 289-99.

172 Cohn BA. In search of human skin pheromones. Arch Dermatol 1994; 130: 1048-51. 
173 Kindred B. Selection for an invariant character, vibrissa number in the house mouse. V. Selection on non-tabby segregants from tabby selection lines. Genetics 1967; 55: 365-73.

174 Tabata T, Kornberg TB. Hedgehog is a signaling protein with a key role in patterning Drosophila imaginal discs. Cell 1994; 76: 89-102.

175 Parr BA, McMahon AP. Dorsalizing signal Wnt-7a required for normal polarity of D-V and A-P axes of mouse limb. Nature 1995; 374: 350-3.

176 Dreyer SD, Zhou G, Baldini A et al. Mutations in LMX1B cause abnormal skeletal patterning and renal dysplasia in nail patella syndrome. Nat Genet 1998; 19: 47-50.

177 Akam M. The molecular basis for metameric pattern in the Drosophila embryo. Development 1987; 101: 1-22.

178 Gaul U, Jackle H. Analysis of maternal effect mutant combinations elucidates regulation and function of the overlap of hunchback and Kruppel gene expression in the Drosophila blastoderm embryo. Development 1989; 107: 651-62.

179 Ingham PW, Hidalgo A. Regulation of wingless transcription in the Drosophila embryo. Development 1993; 117: 283-91.

180 Altschul SF, Madden TL, Schaffer AA et al. Gapped BLAST and PSIBLAST: a new generation of protein database search programs. Nucleic Acids Res 1997; 25: 3389-402.

181 Luke GN, Holland PW. Amphioxus type I keratin cDNA and the evolution of intermediate filament genes. J Exp Zool 1999; 285: 50-6.

182 Hutchison CJ. Lamins: building blocks or regulators of gene expression? Nat Rev Mol Cell Biol 2002; 3: 848-58.

183 Gupta RS, Golding GB. The origin of the eukaryotic cell. Trends Biochem Sci 1996; 21: 166-71.

184 Cohen M, Lee KK, Wilson KL et al. Transcriptional repression, apoptosis, human disease and the functional evolution of the nuclear lamina. Trends Biochem Sci 2001; 26: 41-7.

185 Karabinos A, Schunemann J, Meyer $\mathrm{M}$ et al. The single nuclear lamin of Caenorhabditis elegans forms in vitro stable intermediate filaments and paracrystals with a reduced axial periodicity. J Mol Biol 2003; 325: 241-7. 
186 Riemer D, Dodemont H, Weber K. A nuclear lamin of the nematode Caenorhabditis elegans with unusual structural features; cDNA cloning and gene organization. Eur J Cell Biol 1993; 62: 214-23.

187 Terron-Kwiatkowski A, Paller AS, Compton J et al. Two cases of primarily palmoplantar keratoderma associated with novel mutations in keratin 1. $J$ Invest Dermatol 2002; 119: 966-71.

188 Hatsell SJ, Eady RA, Wennerstrand L et al. Novel splice site mutation in keratin 1 underlies mild epidermolytic palmoplantar keratoderma in three kindreds. J Invest Dermatol 2001; 116: 606-9.

189 Winter H, Rogers MA, Langbein L et al. Mutations in the hair cortex keratin hHb6 cause the inherited hair disease monilethrix. Nat Genet 1997; 16: 372-4.

190 Winter H, Rogers MA, Gebhardt M et al. A new mutation in the type II hair cortex keratin hHb1 involved in the inherited hair disorder monilethrix. Hum Genet 1997; 101: 165-9.

191 Green KJ, Gaudry CA. Are desmosomes more than tethers for intermediate filaments? Nat Rev Mol Cell Biol 2000; 1: 208-16.

192 McGrath JA. Hereditary diseases of desmosomes. J Dermatol Sci 1999; 20: 85-91.

193 Jamora C, Fuchs E. Intercellular adhesion, signalling and the cytoskeleton. Nat Cell Biol 2002; 4: E101-8.

194 Kitajima Y. Mechanisms of desmosome assembly and disassembly. Clin Exp Dermatol 2002; 27: 684-90.

195 Shibata T, Gotoh M, Ochiai A et al. Association of plakoglobin with APC, a tumor suppressor gene product, and its regulation by tyrosine phosphorylation. Biochem Biophys Res Commun 1994; 203: 519-22.

196 Maeda O, Usami N, Kondo M et al. Plakoglobin (gamma-catenin) has TCF/LEF family-dependent transcriptional activity in beta-catenin-deficient cell line. Oncogene 2004; 23: 964-72.

197 Karnovsky A, Klymkowsky MW. Anterior axis duplication in Xenopus induced by the over-expression of the cadherin-binding protein plakoglobin. Proc Natl Acad Sci U S A 1995; 92: 4522-6.

198 Hakimelahi S, Parker HR, Gilchrist AJ et al. Plakoglobin regulates the expression of the anti-apoptotic protein BCL-2. J Biol Chem 2000; 275: 10905-11. 
199 North AJ, Bardsley WG, Hyam J et al. Molecular map of the desmosomal plaque. J Cell Sci 1999; 112: 4325-36.

200 Hanakawa Y, Matsuyoshi N, Stanley JR. Expression of desmoglein 1 compensates for genetic loss of desmoglein 3 in keratinocyte adhesion. $J$ Invest Dermatol 2002; 119: 27-31.

201 Steijlen PM, van Steensel MA, Jansen BJ et al. Cryptic splicing at a nonconsensus splice-donor in a patient with a novel mutation in the plakophilin-1 gene. J Invest Dermatol 2004; 122: 1321-4.

202 Bierkamp C, McLaughlin KJ, Schwarz H et al. Embryonic heart and skin defects in mice lacking plakoglobin. Dev Biol 1996; 180: 780-5.

203 Gallicano GI, Kouklis P, Bauer C et al. Desmoplakin is required early in development for assembly of desmosomes and cytoskeletal linkage. J Cell Biol 1998; 143: 2009-22.

204 Kljuic A, Bazzi H, Sundberg JP et al. Desmoglein 4 in hair follicle differentiation and epidermal adhesion: evidence from inherited hypotrichosis and acquired pemphigus vulgaris. Cell 2003; 113: 249-60.

205 McGrath JA, McMillan JR, Shemanko CS et al. Mutations in the plakophilin 1 gene result in ectodermal dysplasia/skin fragility syndrome. Nat Genet 1997; 17: $240-4$.

206 Norgett EE, Hatsell SJ, Carvajal-Huerta L et al. Recessive mutation in desmoplakin disrupts desmoplakin-intermediate filament interactions and causes dilated cardiomyopathy, woolly hair and keratoderma. Hum Mol Genet 2000; 9: 2761-6.

207 McKoy G, Protonotarios N, Crosby A et al. Identification of a deletion in plakoglobin in arrhythmogenic right ventricular cardiomyopathy with palmoplantar keratoderma and woolly hair (Naxos disease). Lancet 2000; 355: 2119-24.

208 Bauer R, Lehmann C, Fuss B et al. The Drosophila gap junction channel gene innexin 2 controls foregut development in response to Wingless signalling. $J$ Cell Sci 2002; 115: 1859-67.

209 Dermietzel R, Hwang TK, Spray DS. The gap junction family: structure, function and chemistry. Anat Embryol 1990; 182: 517-28. 
210 Kikuchi T, Adams JC, Miyabe Y et al. Potassium ion recycling pathway via gap junction systems in the mammalian cochlea and its interruption in hereditary nonsyndromic deafness. Med Electron Microsc 2000; 33: 51-6.

211 Kikuchi T, Kimura RS, Paul DL et al. Gap junction systems in the mammalian cochlea. Brain Res Brain Res Rev 2000; 32: 163-6.

212 Severs NJ. Pathophysiology of gap junctions in heart disease. J Cardiovasc Electrophysiol 1994; 5: 462-75.

213 Fischbeck KH, Abel A, Lin GS et al. X-linked Charcot-Marie-Tooth disease and connexin32. Ann N Y Acad Sci 1999; 883: 36-41.

214 Evans WH, Martin PE. Gap junctions: structure and function (Review). Mol Membr Biol 2002; 19: 121-36.

215 van Steensel MA, Jonkman MF, van Geel M et al. Clouston syndrome can mimic pachyonychia congenita. J Invest Dermatol 2003; 121: 1035-8.

216 van Steensel MA, van Geel M, Nahuys M et al. A novel connexin 26 mutation in a patient diagnosed with keratitis- ichthyosis-deafness syndrome. $J$ Invest Dermatol 2002; 118: 724-7.

217 Richard G, Rouan F, Willoughby CE et al. Missense Mutations in GJB2 Encoding Connexin-26 Cause the Ectodermal Dysplasia Keratitis-IchthyosisDeafness Syndrome. Am J Hum Genet 2002; 70: 5.

218 van Geel M, van Steensel MA, Kuster W et al. HID and KID syndromes are associated with the same connexin 26 mutation. Br J Dermatol 2002; 146: 938-42.

219 Van Steensel MA, Steijlen PM, Bladergroen RS et al. A Phenotype Resembling the Clouston Syndrome with Deafness Is Associated with a Novel Missense GJB2 Mutation. J Invest Dermatol 2004; 123: 291-3.

220 Rouan F, White TW, Brown N et al. trans-dominant inhibition of connexin-43 by mutant connexin-26: implications for dominant connexin disorders affecting epidermal differentiation. J Cell Sci 2001; 114: 2105-13.

221 Smahi A, Courtois G, Rabia SH et al. The NF-kappaB signalling pathway in human diseases: from incontinentia pigmenti to ectodermal dysplasias and immune-deficiency syndromes. Hum Mol Genet 2002; 11: 2371-5.

222 Lamartine J, Munhoz Essenfelder G, Kibar Z et al. Mutations in GJB6 cause hidrotic ectodermal dysplasia. Nat Genet 2000; 26: 142-4. 
223 Glade C, van Steensel MA, Steijlen PM. Hypotrichosis, lymphedema of the legs and acral telangiectasias--new syndrome? Eur J Dermatol 2001; 11: 5157.

224 Irrthum A, Devriendt K, Chitayat D et al. Mutations in the transcription factor gene SOX18 underlie recessive and dominant forms of hypotrichosislymphedema-telangiectasia. Am J Hum Genet 2003; 72: 1470-8.

225 Hosking BM, Wang SC, Downes $\mathrm{M}$ et al. The VCAM-1 gene that encodes the vascular cell adhesion molecule is a target of the Sry-related high mobility group box gene, Sox18. J Biol Chem 2004; 279: 5314-22.

226 Zorn AM, Barish GD, Williams BO et al. Regulation of Wnt signaling by Sox proteins: XSox17 alpha/beta and XSox3 physically interact with beta-catenin. Mol Cell 1999; 4: 487-98.

227 Roman Corona-Rivera J, Corona-Rivera E, Fragoso-Herrera R et al. Probable new syndrome in a Mexican family with congenital palmar polyonychia and postaxial limb defects. Am J Med Genet 2004; 125A: 205-9.

228 Van Steensel MA, Van Geel M, Steijlen PM. New syndrome of hypotrichosis, striate palmoplantar keratoderma, acro-osteolysis and periodontitis not due to mutations in cathepsin C. Br J Dermatol 2002; 147: 575-81.

229 Toomes C, James J, Wood AJ et al. Loss-of-function mutations in the cathepsin $\mathrm{C}$ gene result in periodontal disease and palmoplantar keratosis [see comments]. Nat Genet 1999; 23: 421-4.

230 Gorlin RJ. Of palms, soles, and gums. J Med Genet 2000; 37: 81-2.

231 Maroteaux PL, M. The malady of Toulouse-Lautrec. J.A.M.A. 1965; 191: 7157.

232 Gelb BD, Shi GP, Chapman HA et al. Pycnodysostosis, a lysosomal disease caused by cathepsin K deficiency. Science 1996; 273: 1236-8.

233 Katunuma N, Matsunaga Y, Saibara T. Mechanism and regulation of antigen processing by cathepsin B. Adv Enzyme Regul 1994; 34: 145-58.

234 Bennett K, Levine T, Ellis JS et al. Antigen processing for presentation by class II major histocompatibility complex requires cleavage by cathepsin E. Eur J Immunol 1992; 22: 1519-24.

235 Stefanovic J, Ferencik M, Kotulova D et al. Interaction of intracellular proteases and immune mechanisms. Acta Biol Med Ger 1977; 36: 1955-8. 
236 Egelrud T. Desquamation in the stratum corneum. Acta Derm Venereol Suppl (Stockh) 2000; 208: 44-5.

237 Horikoshi T, Igarashi S, Uchiwa H et al. Role of endogenous cathepsin D-like and chymotrypsin-like proteolysis in human epidermal desquamation. $\mathrm{Br} J$ Dermatol 1999; 141: 453-9.

238 Suzuki Y, Koyama J, Moro O et al. The role of two endogenous proteases of the stratum corneum in degradation of desmoglein-1 and their reduced activity in the skin of ichthyotic patients. Br J Dermatol 1996; 134: 460-4.

239 Babloyantz A, North Atlantic Treaty Organization. Scientific Affairs Division. Self-organization, emerging properties, and learning. New York: Plenum Press, 1991.

240 Wong S, Witte ON. The BCR-ABL story: bench to bedside and back. Annu Rev Immunol 2004; 22: 247-306.

241 Tamura K, Fukuoka M. Molecular target-based cancer therapy: tyrosine kinase inhibitors. Int J Clin Oncol 2003; 8: 207-11.

242 Blagosklonny MV. Gefitinib (iressa) in oncogene-addictive cancers and therapy for common cancers. Cancer Biol Ther 2004; 3: 436-40.

243 Van Steensel M. Does progressive symmetric erythrokeratoderma exist? Br J Dermatol 2004; 150: 1043-5.

244 Sangkuhl K, Schulz A, Rompler H et al. Aminoglycoside-mediated rescue of a disease-causing nonsense mutation in the V2 vasopressin receptor gene in vitro and in vivo. Hum Mol Genet 2004; 13: 893-903.

245 Choo-Kang LR, Zeitlin PL. Type I, II, III, IV, and V cystic fibrosis transmembrane conductance regulator defects and opportunities for therapy. Curr Opin Pulm Med 2000; 6: 521-9.

246 Lin N, Wu B, Jansen R et al. Information assessment on predicting proteinprotein interactions. BMC Bioinformatics 2004; 5: 154.

247 Kumar R, Conklin DS, Mittal V. High-throughput selection of effective RNAi probes for gene silencing. Genome Res 2003; 13: 2333-40.

248 Bitner M, Skelton G. Low cost, highly effective parallel computing achieved through a Beowulf cluster. Biomed Sci Instrum 2003; 39: 42-7.

249 Ramirez-Solis R, Zheng H, Whiting J et al. Hoxb-4 (Hox-2.6) mutant mice show homeotic transformation of a cervical vertebra and defects in the closure of the sternal rudiments. Cell 1993; 73: 279-94. 
250 van Steensel M, Smith FJ, Steijlen PM et al. The gene for hypotrichosis of Marie Unna maps between D8S258 and D8S298: exclusion of the hr gene by cDNA and genomic sequencing. Am J Hum Genet 1999; 65: 413-9. 
Summary 
Most of our knowledge of human molecular biology stems from the study of human monogenic diseases, as demonstrated in this review. In effect, monogenic disorders are Nature's equivalent of our mouse transgenic, knockout and "knock in" (or hit-and-run mutant ${ }^{249}$ ) systems. Their study has been and will be most rewarding in terms of understanding gained of complex biological processes. In this thesis, the results obtained during five years of studying rare hereditary hair and skin disorders are described. The project originated as a linkage analysis in a family with hypotrichosis congenita of Marie Unna (HMU, MIM \#146550), a rare autosomal dominant disorder characterized by early loss of wiry scalp hair and generalized hypotrichosis, affecting both sexes equally ${ }^{250}$. Because we had access to a large pedigree, we chose to analyze HMU. The hair loss resembles male pattern baldness and HMU is considered as a monogenic model for the latter disorder. Finding the cause should provide significant insight into hair biology and the causes of hereditary and perhaps acquired hair loss. The causative gene proved elusive. We have now mapped the critical region on chromosome 8 in great detail, as described in chapter 2, and meanwhile narrowed down the locus to about 360 kilobases, but have not yet found a causative mutation. During our search we studied several other syndromes with hypotrichosis as a prominent symptom, in the hope that these disorders might serve as models and assist in identifying the HMU gene through a candidate gene approach. Several patients turned out to have mutations in gap junction genes, showing unexpectedly that gap junction communication is crucial for the maintenance and proper development of hair follicles and nails. As is shown in chapters 3 to 9, we were successful in finding novel connexin mutations, which has resulted in the initiation of functional studies in our lab since we wished to elucidate the intriguing diseases caused by mutations in gap junction genes. Chapter 10 reports our findings in a patient suffering from a rare desmosome disorder, whose unexpectedly mild phenotype led to the discovery of an unusual genetic mechanism that may one day be useful for gene therapy. Other patients, described in chapters 11 
through 15 , had diseases that were either extremely rare or had never even been described before. The patients who are suffering from diseases that have not yet been elucidated and who are described in the other chapters will hopefully one day make their contribution to our understanding of skin biology. One disease that we described first, the hypotrichosis-lymphedematelangiectasia syndrome, has now been elucidated, demonstrating the importance of SRY-box containing proteins for human and murine hair development and proving that key developmental genes can be identified through the careful clinical study of "new" syndromes. 
Samenvatting 
Bijna alles wat we momenteel weten van menselijke haarbiologie komt voort uit de studie van erfelijke haarziekten. Voor geïnteresseerde artsen en biologen is het haar meer dan alleen een decoratief aanhangsel van de huid. De haarfollikel (ook bekend als haarzakje) is een miniatuur orgaantje waarin alle moleculaire processen die belangrijk zijn bij groei, ontwikkeling en (cel)dood zich afspelen. Door de ligging in de huid is de haarfollikel een aantrekkelijk studie-object. Zij is eenvoudig bereikbaar voor microscopisch of genetisch onderzoek. Bovendien zijn afwijkingen in het normaal functioneren ook altijd aan de buitenkant zichtbaar. Dit geldt voor de gehele huid en de dermatologie is voor de moleculair genetisch en biologisch geïnteresseerde arts dan ook een ideaal vakgebied.

Dit proefschrift beschrijft de resultaten van bijna vijf jaar klinisch en vooral ook moleculair genetisch onderzoek aan menselijke erfelijke haarziekten. Het gaat veelal om zeldzame aandoeningen, die vaak niet alleen het haar treffen maar ook de huid zelf en andere huidaanhangsels zoals zweetklieren en nagels. Het onderzoek begon met koppelingsonderzoek bij hypotrichosis congenita of Marie Unna (HMU, MIM \#146550), een zeldzame aangeboren haarafwijking gekenmerkt door grof, staaldraad-achtig hoofdhaar dat in de loop van de jaren uitvalt, terwijl overige lichaamsbeharing niet of nauwelijks aanwezig is. Mannen en vrouwen zijn aangedaan ${ }^{250}$. Wij kozen voor HMU omdat we toegang hadden tot grote families voor genetische analyse. Het partoon van haarverlies lijkt op dat bij mannelijke kaalheid en HMU wordt dan ook beschouwd als een model voor deze laatste aandoening. Het vinden van het oorzakelijk gen voor HMU zou dan ook inzicht kunnen verschaffen in de moleculaire biologie van de haarfollikel en in het bijzonder in de oorzaak van mannelijk patroon kaalheid. Het causaal gen is echter nog altijd niet geïdentificeerd. Zoals beschreven in hoofdstuk 2 hebben we de plaats waar het oorzakelijk defect moet liggen in kaart gebracht en intussen de omvang van het gebied teruggebracht tot circa 350 kilobases. Tijdens onze zoektocht hebben wij andere aandoeningen met hypotrichosis bestudeerd, deels in de hoop dat deze zouden kunnen dienen als model voor HMU en ingangen 
zouden opleveren voor een kandidaatgen-benadering. Verschillende patiënten bleken mutaties te hebben in zogeheten gap junction genen die coderen voor communicatie eiwitten, de connexines. Onverwachts bleek aldus dat connexines essentiëel zijn voor een normale groei en ontwikkeling van haren en nagels. Zoals beschreven in hoofdstukken 3-9 hebben wij tal van niet eerder beschreven mutaties in verschillende gap junction genen gevonden. Inmiddels zijn in het laboratorium voor experimentele dermatologie functionele studies gestart die als doel hebben op te helderen waarom mutaties in connexines leiden tot de verschillende afwijkingen aan huid, haren en nagels. Al doende vindt men zo nu en dan wat onverwachts. Zo wordt in hoofdstuk 10 een patiënt beschreven die lijdt aan een zeldzame aandoening van de desmosomen. Dit zijn eiwitcomplexen die onder andere huidcellen bij elkaar houden. Onze patiënt had een onverwacht mild ziektebeeld in vergelijking met wat eerder was beschreven en we hebben laten zien dat dit het gevolg is van een vorm van gen-therapie waarbij door een speciaal genetisch mechanisme het gendefect wordt ondervangen.

Zoals beschreven in hoofdstuk 11 tot en met 15 hebben wij bovendien een aantal "nieuwe" en een zeer zeldzaam syndroom beschreven. Het nut van dit soort beschrijvingen voor genetisch onderzoek is vooral gelegen in het feit dat het bestaan van een niet eerder beschreven ziekte bewijst dat er nog niet bekende genen betrokken zijn bij de aanleg van de in de ziekte betrokken organen. Een nauwkeurige klinische beschrijving is als het oorzakelijk gendefect eenmaal is gevonden van groot belang om te begrijpen wat het gevonden eiwit nu eigenlijk doet. Een goed voorbeeld is het hypotrichosislymphedema-telangiectasia (HLT) syndroom, dat door ons enige jaren geleden voor het eerst is beschreven. De symptomen zijn kaalheid, lymfoedeem en afwijkingen aan kleine en grotere bloedvaten; soms ook zijn er hartafwijkingen. De ziekte blijkt te worden veroorzaakt door mutaties in het gen SOX18. Dit codeert voor een zogeheten SRY-box eiwit. Deze klasse eiwitten is onder andere betrokken bij de groei van het skelet en de geslachtelijke ontwikkeling, maar het was nog niet bekend dat ze ook 
betrokken zijn bij de ontwikkeling van haren en kleine bloedvaten in de huid. Doordat dit ziektebeeld werd afgegrensd konden vervolgens meer patiënten worden geïdentificeerd waarna het oorzakelijk gen kon worden opgespoord. 


\section{Publications}


Publications in international peer-reviewed journals

1. Maurice van Steensel, George Homminga, Pieter Buma, Frans Olthuis, Wim van den Berg. Optimization of cryopreservative procedures for human articular cartilage chondrocytes. Arch Orthop Trauma Surg 113:318-22; 1994

2. L. Schuman, G.N. Homminga, P. Buma, M.A.M. van Steensel, P.M. van der Kraan, W.B. van den Berg. Biological implants for fullthickness cartilage defects. Clin Rheumatol 12:556-557;1993

3. M.A.M. van Steensel, P. Buma, M.C. de Waal Malefijt, F.H.J. van de Hoogen, H.G. Brunner. Oto-spondylo-megaepiphyseal dysplasia (OSMED): clinical description of three patients homozygous for a COL11A2 mutation. Am J Med Genet 1997;70:315-323

4. M. van Steensel, P.M. Steijlen. Genetics of psoriasis. In: P.C.M. van de Kerkhof (ed.). Management of psoriasis. Clin Dermatol 1997;15:669-75

5. M.A.M. van Steensel, P.M. Steijlen, J Rees. Molecular genetic approaches to skin disease: keratins and keratinisation. J Roy Coll Phys $1997 ; 31: 379-83$

6. M.A.M. van Steensel. Mirror hands and feet. J Med Genet 1997;34:7012

7. M.A.M. van Steensel. Robinow syndrome. J Med Genet 1998;35:349-50

8. M.A.M. van Steensel, R.M. Winter. Databases for clinical geneticists -an overview. Clin Genet 1998;53:323-30 
9. M. A.M. van Steensel, P. M. Steijlen. Hypomelanosis Ito: a symptom, not a syndrome. Am J Med Genet 1998;80:435

10. Van Bokhoven H, Jung M, Smits AP, Van Beersum S, Schendorf F, Van Steensel M, Veenstra M, Tuerlings JH, Mariman EC, Brunner HG, Wienker TF, Reis A, Ropers HH, Hamel BC. Limb Mammary Syndrome: A new genetic disorder with mammary hypoplasia,ectrodactyly, and other hand/foot anomalies maps to human chromosome 3q27. Am J Hum Genet 1999;64:538-546

11. Steijlen PM, van Steensel MAM. Paradominant inheritance: an intriguing hypothesis explaining the familial occurence of sporadic syndromes. Am J Med Genet 1999;85:359-60

12. Van Steensel M, Smith FJ, Steijlen PM, Kluijt I, Stevens HP, Messenger A, Kremer H, Dunnill MG, Kennedy C, Munro CS, Doherty VR, McGrath JA, Covello SP, Coleman CM, Uitto J, McLean WH. The gene for hypotrichosis of Marie Unna maps between D8S258 and D8S298: exclusion of the hr gene by cDNA and genomic sequening. Am J Hum Genet 1999;65:413-19

13. Van Steensel MAM, Celli J, Van Bokhoven H, Brunner HG. Probing the gene eXpression database for candidate genes. Eur J Hum Genet 1999;7:910-9

14. M.A.M. van Steensel, P.M. Steijlen, H.E.Vietor, R. Happle. Sweat testing in hypomelanosis of Ito: divergent results reflect genetic heterogeneity. Eur J Dermatol 2000;10:217-9 
15. M.A.M. van Steensel, R. Happle, P.M. Steijlen. Molecular genetics of the hair follicle: the state of the art. Proc Soc Exp Biol Med 2000;223:1-7.

16. M.A.M. van Steensel, F.J. Smith, B.C.J. Hamel, P.M. Steijlen. A new type of pachyonychia congenita. Eur J Dermatol 2001;12:188-91

17. M.A.M. van Steensel, N.G. Jaspers, P.M. Steijlen. A case of Rombo syndrome. Br J Dermatol 2001;144:1215-8

18. M.A.M. van Steensel, M.I. Koedam, O.Q.W. Swinkels, F. Rietveld, P.M. Steijlen. Woolly hair, premature loss of teeth, nail dystrophy, acral hyperkeratosis and facial abnormalities: possible new syndrome in a Dutch kindred. Br J Dermatol 2001;145:157-62,

19. L. Laino, M.A.M. van Steensel, D. Innocenzi, G. Camplone. Nevus sebaceus in sibs - a case of paradominant inheritance? Eur J Dermatol 2001;11:97-98

20. M.A.M. van Steensel, M. van Geel, P.M. Steijlen. The molecular basis of hair growth. Eur J Dermatol 2001;11:348-52

21. C. Glade, M.A.M. van Steensel, P.M. Steijlen. Hypotrichosis, lymphedema of the legs and acral telangiectasias -New syndrome? Eur J Dermatol 2001:11;515-517.

22. M.A.M. van Steensel, M. van Geel, P.M. Steijlen. New syndrome of hypotrichosis, striate palmoplantar keratoderma, acro-osteolysis and peridontitis not due to mutations in cathepsin C. Br J Dermatol 2002;147:575-81 
23. M.A.M. van Steensel, M. van Geel, P.M. Steijlen. Mal de Meleda without mutations in the ARS coding sequence. Eur J Dermatol, 2002;12:129-32

24. M. van Geel, M.A.M. van Steensel, P.M. Steijlen. Connexin 30.3 is not required for normal skin function in humans. $\mathrm{Br} J$ Dermatol 2002;147:1257-56

25. M.A.M. van Steensel, M. van Geel, M. Nahuys, J.H. Sillevis Smitt, P.M. Steijlen. A novel connexin26 mutation in a patient diagnosed with keratitis-ichthyosis-deafness syndrome. J Invest Dermatol 2002;118:743-7

26. Takahiro Hamada, W. H. Irwin McLean, Michele Ramsay, Gabrielle H. S. Ashton, Arti Nanda, Trefor Jenkins, Isobel Edelstein, Andrew P. South, Oliver Bleck, Vesarat Wessagowit, Rajeev Mallipeddi, Guy E. Orchard, Hong Wan, Patricia J. C. Dopping-Hepenstal, Jemima E. Mellerio, Neil V. Whittock, Colin S. Munro, Maurice A. M. van Steensel, Peter M. Steijlen, Jian Ni, Lurong Zhang, Takashi Hashimoto, Robin A. J. Eady, and John A. McGrath. Lipoid proteinosis maps to 1q21 and is caused by mutations in the extracellular matrix protein 1 gene (ECM1). Hum Mol Genet 2002;11: 833-840

27. Van Geel M., van Steensel M.A.M, Kuster W, Hennies, H. C., Happle R, Steijlen P. M., Konig, A. HID and KID syndromes are associated with the same connexin 26 mutation. Br J Dermatol 2002;146: 938-42.

28. M.A.M. van Steensel, M.F. Jonkman, M. van Geel, P.M. Steijlen, W.H.I. McLean, F.J.D. Smith Clouston syndrome can mimic pachyonychia congenita. J Invest Dermatol 2003;121:1035-38 
29. Irrthum A, Devriendt K, Chitayat D, Matthijs G, Mertens A, Glade C, Steijlen PM, Fryns J-P, van Steensel MAM, Vikkula M. Mutations in the transcription factor SOX18 underlie recessive and dominant forms of lymphedema with alopecia and telangiectasia. Am J Hum Genet $2003 ; 72: 1470-8$

30. W. Vissers, M.A.M. van Steensel, W.O. Reinier, P.M. Steijlen,C v.d. Vleuten Klippel-Trenaunay syndrome and Sturge-Weber syndrome: Variations on a theme? Eur J Dermatol, 2003:13:238-41

31. W. H. Irwin McLean, Alan D. Irvine, Kevin J. Hamill, Neil V. Whittock, Carrie M. Coleman-Campbell1, Jemima E. Mellerio, Gabrielle S. Ashton, Patricia J. H. Dopping-Hepenstal, Robin A. J. Eady, Tanvir Jamil, Rodney J. Phillip, S. Ghulam Shabbir, Tahir S. Haroon, Khawar Khurshid, Jonathan E. Moore, Brian Page, Jonathan Darling, David J. Atherton, Maurice A. M. van Steensel, Colin S. Munro, Frances J. D. Smith and John A. McGrath. An unusual N-terminal deletion of the laminin a3a isoform leads to the chronic granulation tissue disorder laryngo-onycho-cutaneous syndrome. Hum Mol Genet 2003; 12:23952409

32. Peter M. Steijlen, Maurice A. M. van Steensel, Bastiaan J.H. Jansen, Willeke Blokx, Peter C. M. van de Kerkhof, Rudolf Happle, Michel van Geel. Cryptic splicing at a non-consensus splice-donor site in a patient with a new mutation in the plakophilin-1 gene. J Invest Dermatol 2004;122:1321-3

33. M.A.M. van Steensel. Poland anomaly: not unilateral or bilateral but a mosaic. Am J Med Genet 2004;125A(2):211-2 
34. Laino L, Pala S, Innocenzi D, Accappaticcio G, van Steensel M.A.M. Genital porokeratosis in a negroid male. Eur J Dermatol 2004;4:190-2

35. C. Tilli, M.A.M. van Steensel, G.A.M. Krekels, H.A.M. Neumann, F.C.M. Ramaekers. Molecular pathology of basal cell carcinoma. Br J Dermatol, In Press

36. A. Brun, M.A.M. van Steensel. A third case of HOPP syndrome. Confirmation of the phenotype. Br J Dermatol 2004;150:1032-3

37. M.A.M. van Steensel. Does progressive symmetric erythrokeratoderma exist? Br J Dermatol 2004;150:1043-5

38. M.A.M. van Steensel, P.M. Steijlen, R.S. Bladergroen, L. Hoefsloot, C.M. van Ravenswaaij-Arts, M. van Geel. A Clouston-like phenotype with deafness is associated with a novel GJB2 missense mutation. J Invest Dermatol 2004;123:291-3

39. M.A.M. van Steensel. Why minocycline can cause systemic lupus - a hypothesis and suggestions for therapeutic interventions based on it. Med Hypoth 2004;63:31-34

40. M.A.M. van Steensel, M. van Geel, P.M. Steijlen. Hair and nail disease. Am J Med Genet 2004;131C(1):52-60

41. M.A.M. van Steensel. Gap junction skin disease. Am J Med Genet 2004;131C(1):12-9

42. M.A.M. van Steensel, M.B. Maessen-Visch, P.M. Steijlen. A new type of twin spot. Am J Med Genet, In Press 
43. M.A.M. van Steensel, M. van Geel, P.M. Steijlen. A novel type of erythrokeratoderma. Br J Dermatol, In Press

44. M.A.M. van Steensel, I. Van de Burgt, R.S. Bladergroen, P.M. Steijlen, M. Vermeer, M. van Geel. A 2-bp deletion in the GJA1 gene is associated with oculo-dento-digital dysplasia with palmoplantar keratoderma. Am J Med Genet, In Press

45. M.A.M. van Steensel. Some twin spots may not be twins. Am J Med Genet, In Press

46. C. G. Bauland, M.A.M. van Steensel, P.M. Steijlen, P.N.M.A. Rieu, P.H.M Spauwen. The pathogenesis of hemangioma. In Press

47. M.A.M. van Steensel, M. van Geel. Does autosomal recessive erythrokeratoderma variabilis exist? J Invest Dermatol, In Press

48. M.A.M. van Steensel, P.M. Steijlen, R.S. Bladergroen, M.H. Vermeer, P.M. Steijlen. A missense mutation in the type II hair keratin $\mathrm{hHb} 3$ is associated with monilethrix. J Med Genet, In Press.

49. M.A.M. van Steensel, C. de Die-Smulders, P.M. Steijlen. Myhre syndrome in a female with novel symptoms. In Preparation

50. V.L.R.M. Verstraeten, M.A.M. van Steensel, N. Smeets, M. van Geel. A novel deletion mutation in Birt-Hogg-Dubé syndrome presenting with trichodiscoma. In Preparation

51. M.A.M. van Steensel, D. Marcus-Soekarman. Zimmermann-Laband syndrome in an adult female. In Preparation 
Publications in Dutch journals

1. P.M. Steijlen, M.A.M. van Steensel. Genodermatosen. Ned Tijdschr Derm Venereol 1998;8:207-8

2. Steensel MAM van, Geel M van, Steijlen PM. Het Haim-Munk-plussyndrom. Ned Tijdschr Dermatol Venereol 2001; 11: 123-124

3. P.M. Steijlen, M.A.M. van Steensel. Virologie van de huid. In "Bijblijven", 2000

4. Steensel MAM van, Koedam MI, Swinkels OQ, Rietveld F, Steijlen PM. Kroeshaar, vroegtijdig verlies van tanden, dystrofische nagels, hyperkeratose en dysmorfe facies: nieuw syndroom in een Nederlandse familie. Ned Tijdschr Dermatol Venereol 2001; 11: 116-118

5. Steensel MAM van, Jaspers NGJ, Steijlen PM. Het Rombo-syndroom. Ned Tijdschr Dermatol Venereol 2001; 11: 121-122

6. P.M. Steijlen, M.A.M. van Steensel. De palmoplantaire keratodermieën. Ned Tijdschr Dermatol Venereol 2003,13:87-89

7. J.W.M. Engelen, M. van Steensel, S.G.M.A. Pasmans. Autosomaal recessieve cutis laxa met congenitaal longemfyseem. Ned Tijdschr Dermatol Venereol 2003;13:40-41

8. P.M. Steijlen, M.A.M. van Steensel, P.N.M.A. Rieu. Diagnostiek en behandeling van congenitale vasculaire malformaties. Ned Tijdschr Dermatol Venereol 2004;14:129-34 


\section{Book chapters}

1. P.M. Steijlen, M.A.M. van Steensel, H-J Sillevis-Smit. Huidziekten. In: H.G.Brunner, N. Leschot (eds), Klinische Genetica. Utrecht: Bunge, 1998

2. P.M. Steijlen, M.A.M. van Steensel. Genetische syndromen. In: De Waard-Van der Spek FB, Arnolf P. Oranje (eds), Diagnostiek en therapie in de kinderdermatologie. De Weezenlanden series 25, 1999, pp 89-93

3. M.A.M. van Steensel, P.M. Steijlen. Keratin disorders. In Harper JF, Oranje AP, Prose NS, (eds): "Textbook of Pediatric Dermatology" Blackwell Scientific, 2000, 2004

4. P.M. Steijlen, M.A.M. van Steensel, P. Rieu Vasculaire malformaties. In: Handboek Flebologie. Neuman M et al. Eds, Lemma BV, Utrecht, 2003

5. M.A.M. van Steensel, G.P.H. Lucker, P.M. Steijlen. The palmoplantar keratodermas. In Harper JF, Oranje AP, Prose NS (eds): “Textbook of Pediatric Dermatology" Blackwell Scientific, 2004

6. M.A.M. van Steensel, P.M. Steijlen. Keratin disorders. In Harper JF, Oranje AP, Prose NS, (eds). "Textbook of Pediatric Dermatology" Blackwell Scientific, 2004

7. M.A.M. van Steensel, P.M. Steijlen. Genodermatosen. In: De WaardVan der Spek FB, Arnolf P. Oranje (eds), Diagnostiek en therapie in de kinderdermatologie. In Press 
8. P.M. Steijlen, M.A.M. van Steensel. Ichthyosis. In: De Waard-Van der Spek FB, Arnolf P. Oranje (eds), Diagnostiek en therapie in de kinderdermatologie, In Press

9. M.A.M. van Steensel. A novel disorder characterized by peridontitis and acro-osteolysis. In: Encyclopedic reference of molecular medicine. F. Lange, ed. Springer Verlag, Heidelberg, In Press

10. M.A.M. van Steensel, P.M. Steijlen. Palmoplantarkeratosen. In: Traupe H, (ed). Pädiatrische Dermatologie. Springer Verlag, Heidelberg, In Press

\section{Other}

1. M.A.M. van Steensel. Het syndroom van Robinow. Syllabus for parents and doctors caring for patients with Robinow syndrome.

2. M.A.M. van Steensel, P.C.M. van de Kerkhof, P.M. Steijlen. Ichthyosis. Information for patients. 


\section{Invited lectures/chairs}

1. Basal cell nevus syndrome and Patched. Presentation at Dermatology meeting, december 19, 1996, Nijmegen.

2. The Robinow syndrome: medical aspects. Presentation for Robinow syndrome contact-group at the occasion of its first meeting, 1997.

3. Hypotrichosis congenita Marie Unna. Dept. of Dermatology of Jefferson Medical School Staff meeting, Philadelphia, USA, 1997.

4. Data mining in genetics research. Workshop given at Embryos Genes \& Birth Defects course of the ICH, London, May 5th-7th 1998.

5. The hair follicle as a target for gene therapy. International Gene Therapy Conference, Corfu, August 2001

6. New developments in hair follicle genetics. Introductory lecture and workshop. EHRS, Brussels 2002

7. Hereditary skin disorders. Post-graduate training course for pediatricians, June 2002

8. Hair follicle genetics. Australasian Hair and Wool Research Society, Melbourne 2002

9. Connexins in skin disease. Dermatology Seminars, Glasgow, december 2002

10. Post academic training. Disorders of keratinization, February 2003, Utrecht

11. Chair of developmental genetics session. Continuing medical education of Society of Dutch-speaking dermatologists, March 2003, Brussels

12. Molecular genetics of hair disorders, pediatric dermatology congress, Rotterdam, June 2003

13. Ichthyosis. Dutch skin foundation public education day, November 2003 
14. Connexins and hair disease. Lecture, EADV congress, October 2003, Barcelona

15. Gap junctions and keratins. Lecture, Pachyonychia meeting, February 2004, Park City, Utah

16. Disorders of keratinisation. Genodermatology Task Force meeting, March 2004, Utrecht

17. Advances in palmoplantar keratodermas. EADV Spring Symposium, April 2004, Budapest

18. Neurocutaneous disorders workshop. Van Gelderen symposium workshop, November 2004

19. Neurocutaneous syndromes. Post-academic education for clinical geneticists in training. November 2004

20. Gap junction diseases of the skin. GROW science day, December 2004.

21. Connexin skin diseases. Berliner Stiftung für Dermatologie symposium, December 2004.

Abstracts are not listed. 
Curriculum vitae 
The author was born in Tilburg on September $17^{\text {th }}, 1969$. In grammar school he decided that he wanted to be a geneticist but later thought better of it and set his sights on surgery. From 1981 to 1987, he attended the Gymnasium at the "Cobbenhagen College" in Tilburg. He studied medicine at the University of Nijmegen from 1987 to May, 1996. In 1991 an Erasmus scholarship allowed him to depart to Giessen, Germany, where he worked as a resident in the University Orthopedics Clinic for three months. Here his interest for genetics was re-awakened by a chance encounter with two Russian sisters affected by a spondylo-epiphyseal dysplasia that in retrospect probably was a multiple epihyseal dysplasia. On his return to the Netherlands, he presented himself at the department of Human Genetics in Nijmegen and was given the chance to study Robinow syndrome, a rare skeletal dysplasia. Surgery didn't seem like such a good idea any more. In 1994 and 1995, he worked for six months in the human genetics laboratory of the Catholic University Nijmegen, learning the basic techniques of molecular genetics from dr. Frans Hol by working on the role of platelet-derived growth factor-receptor $\alpha$ (PGDFRA) in the pathogenesis of spina bifida. He also spent half a year doing absolutely nothing whatsoever.

During his residency in Dermatology, the author met dr. (now professor) Peter M. Steijlen, whose enthusiasm for inherited skin disorders proved infectious, resulting in the author's becoming involved in the study of genodermatology. This was kept up even while working in other departments. After the MD exam in 1996, the author was given the opportunity to work with prof. dr. H. G. Brunner in the department of human genetics, Nijmegen. Here he was involved in the development of computerassisted candidate disease gene identification methods. This project took place in close collaboration with the bioinformatics CAOS/CAMM center of Nijmegen University. 
In 1998, the ongoing collaboration with Peter Steijlen resulted in the author's departure to W.H.I. McLean's lab in Philadelphia, USA for a total of two months. Here he worked on linkage analysis in several large pedigrees affected by hypotrichosis congenita Marie Unna and Shabir laryngo-onychocutaneous syndrome. This entailed doing several thousands of polymerase chain reactions and watching many reruns of the Aliens trilogy (now a tetralogy). The linkage analysis in the Marie Unna pedigrees resulted in the identification of the gene locus. A ZON-MW AGIKO project was written using these results and a grant was obtained (920-03-085). With it, the author started his dermatological career as an AGIKO ("assistent geneeskundige in opleiding tot klinisch onderzoeker") in 1999 by working on narrowing down the Marie Unna locus to a manageable size. Industry funding allowed the hiring of a (then) post-doc, dr. Michel van Geel. With his contribution, the experimental work really took off and has now resulted among many other things in this thesis. Another grant brought ing. Reno Bladergroen into the group. He worked on the construction of a gene therapy vector that the author designed together with dr. ir. Bas J.H. Jansen who is now in the Tumor Immunology Laboratory of the University of Nijmegen. In 2003, professor Steijlen became head of the department of Dermatology in Maastricht and the group moved to Maastricht where the author now continues to juggle dermatological training and research. In his spare time, when not being occupied with being a family man, he likes to hack away on UNIX family operating systems (he rewrote the Linux token ring card device driver back in the days when men were still men and wrote their own device drivers), read and watch SF and work on furthering his understanding of karate.

The author is married to Hester Vogel. They have three children: Nathan, Juliette and Samuel. The family also caters to the needs of their long-time feline companion, Annie Pluis van Breukelen. 
Additional figures 


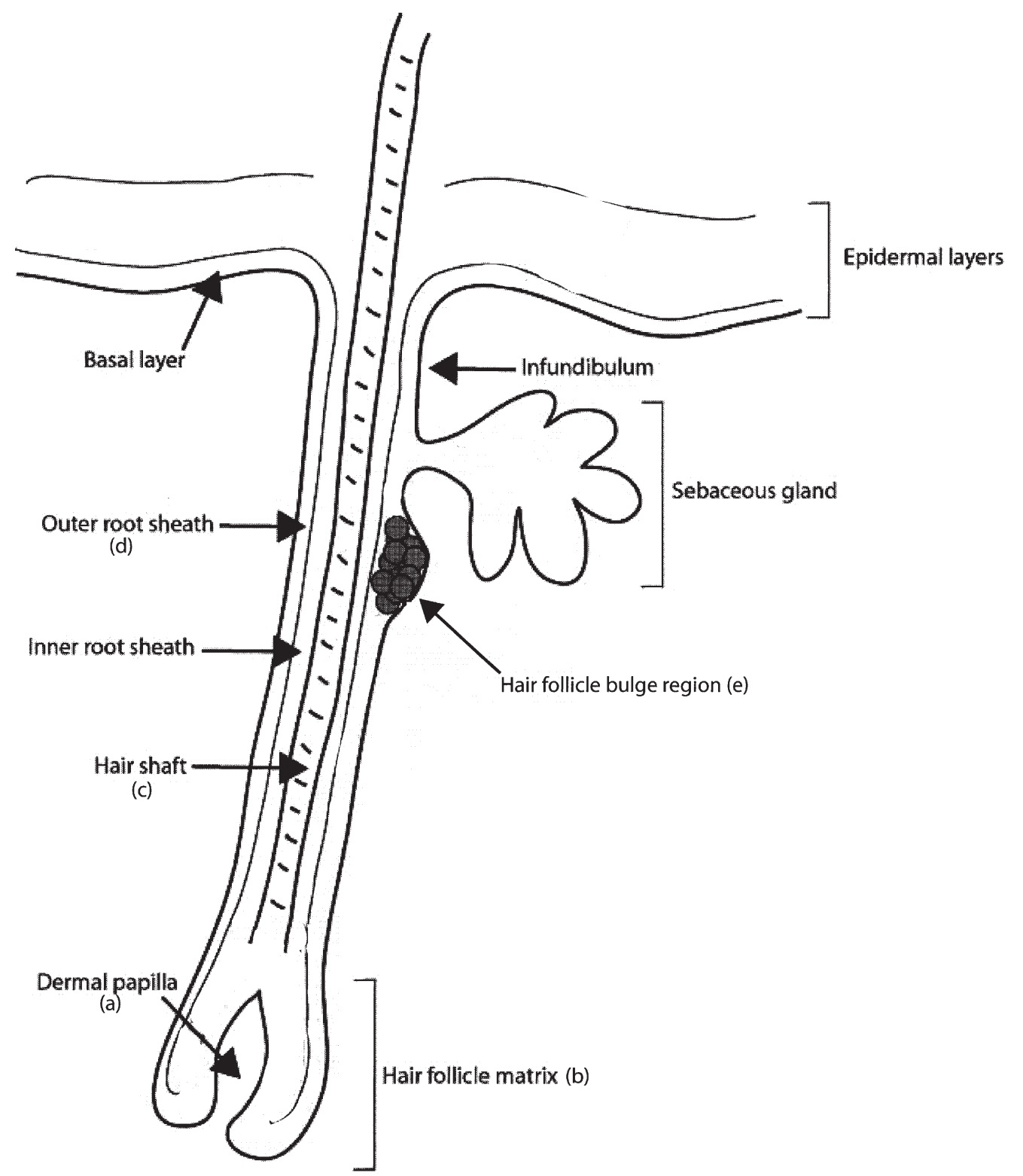

Figure 1. Schematic representation of the hair follicle. Adapted from Alonso \& Fuchs, 2003 


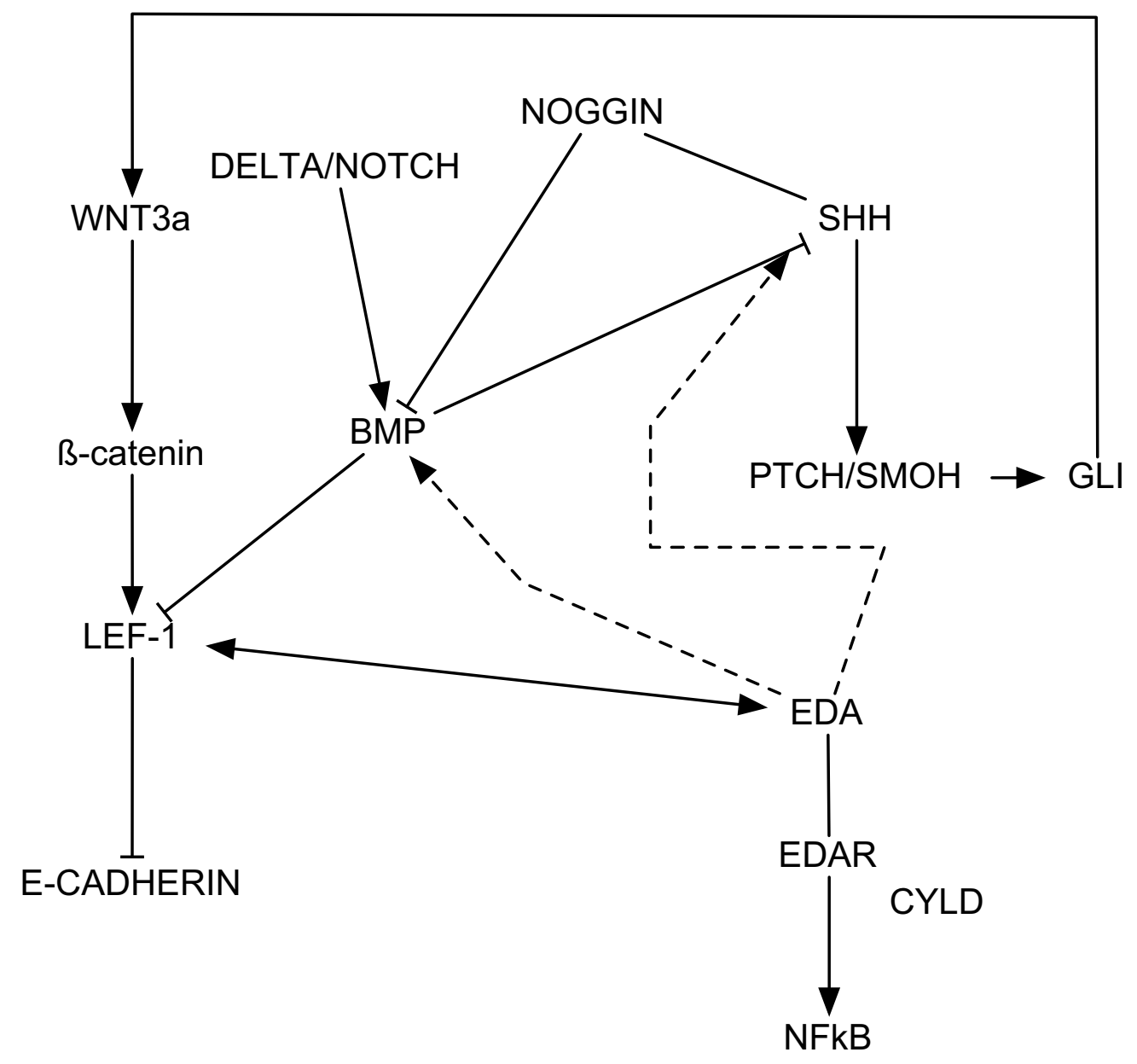

A

B

Figure 2. BMPs and EDA have a central role in hair follicle initiation. The figure demonstrates extensive cross-talk between pathways. Speculative interactions are indicated by dashed lines. 


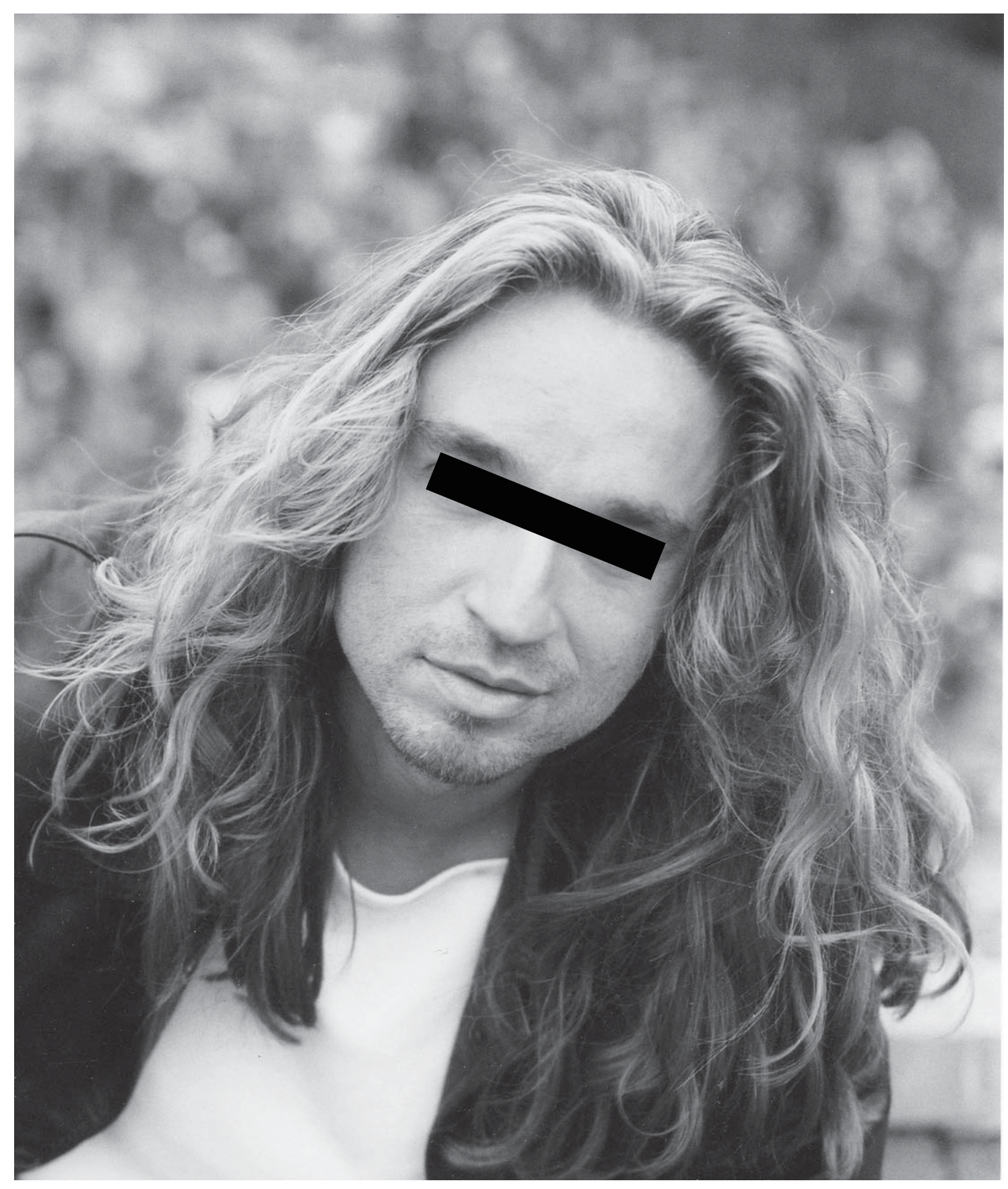

Figure 3. Putative human Angora phenotype 